WATER QUALITY AND HYDROLOGIC DATA FOR THE WEST BRANCH SWIFT RIVER AND EAST BRANCH FEVER BROOK, QUABBIN RESERVOIR DRAINAGE BASIN, MASSACHUSETTS

By Rochelle L. Rittmaster and Gerald G. Girouard

U.S. GEOLOGICAL SUR VEY

Open-File Report 90-182

Prepared in cooperation with the

METROPOLITAN DISTRICT COMMISSION and the MASSACHUSETTS DEPARTMENT OF ENVIRONMENTAL PROTECTION, DIVISION OF WATER POLLUTION CONTROL

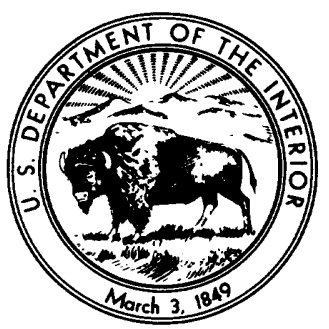

Marlborough, Massachusetts 1992 


\section{U.S. DEPARTMENT OF THE INTERIOR}

MANUEL LUJAN, JR., Secretary

U.S. GEOLOGICAL SURVEY

Dallas L. Peck, Director

For additional information, write to:

\section{District Chief}

Massachusetts - Rhode Island Office

U.S. Geological Survey

Water Resources Division

28 Lord Rd., Suite 280

Marlborough, MA 01752
Copies of this report can be purchased from:

Books and Open-File Reports Section

U.S. Geological Survey

Box 25425, Federal Center

Denver, CO 80225 


\section{CONTENTS}

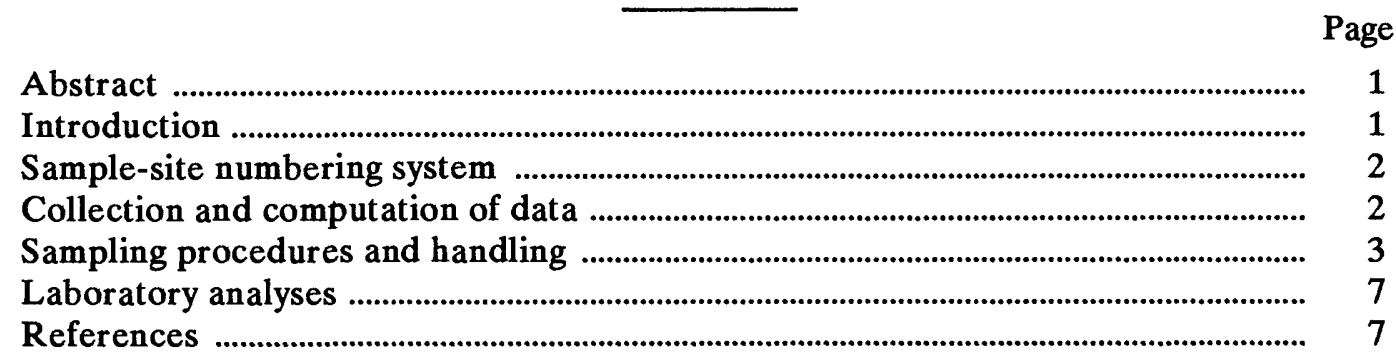

\section{ILLUSTRATIONS}

Figure 1-3. Maps showing:

1. Locations of the West Branch Swift River and East Branch Fever Brook drainage basins, Massachusetts

2. Locations of sampling sites, West Branch Swift River basin, Massachusetts ........... 5

3. Locations of sampling sites, East Branch Fever Brook basin, Massachusetts ......... 6

\section{TABLES}

Table 1. Wet deposition chemistry at station number 423027072204501 , precipitation collector at New Salem, Massachusetts

2. Daily mean discharge at station number 01174565, West Branch Swift River near Shutesbury, Massachusetts

3. Physical characteristics and concentrations of chemical constituents of streamflow at station number 01174565, West Branch Swift River near Shutesbury, Massachusetts

4. Physical characteristics and concentrations of chemical constituents of streamflow at station number 01174555, West Branch Swift River near Cooleyville, Massachusetts

5. Physical characteristics and concentrations of chemical constituents of streamflow at station number 01174560, West Branch Swift River at Cooleyville, Massachusetts

6. Physical characteristics and concentrations of chemical constituents of streamflow at station number 01174563, West Branch Swift River tributary at Cooleyville, Massachusetts 


\section{TABLES (continued)}

Page

7. Physical characteristics and concentrations of chemical constituents of streamflow at station number 01174564, West Branch Swift River tributary 2 at Cooleyville,

Massachusetts

8. Physical characteristics and concentrations of chemical constituents of ground water at station number 422800072232901, Swift River till well near Cooleyville, Massachusetts

9. Physical characteristics and concentrations of chemical constituents of ground water at station number 422803072231801, Swift River sand and gravel well near Cooleyville, Massachusetts

10. Daily mean discharge at station number 01174050, East Branch Fever Brook near Petersham, Massachusetts

11. Physical characteristics and concentrations of chemical constituents of streamflow at station number 01174050, East Branch Fever Brook near Petersham, Massachusetts

12. Physical characteristics and concentrations of chemical constituents of streamflow at station number 01174035, Brooks Pond tributary near Petersham, Massacilusetts

13. Physical characteristics and concentrations of chemical constituents of streamflow at station number 01174040, East Branch Fever Brook below Brooks Pond near Petersham, Massachusetts

14. Physical characteristics and concentrations of chemical constituents of streamflow at station number 01174045, East Branch Fever Brook at West Road near Petersham, Massachusetts

15. Physical characteristics and concentrations of chemical constituents of ground water at station number 422906072124301, Fever Brook till well near Petersham, Massachusetts

16. Physical characteristics and concentrations of chemical constituents of ground water at station number 422910072120501, Knault's well near Petersham, Massachusetts

17. Physical characteristics and concentrations of chemical constituents of ground water at station number 422906072130201, Fever Brook sand and gravel well near Petersham, Massachusetts

18. Description of soil-collection sites and samplings

19. Results of chemical analysis of leachate extractions of soil samples collected in the Swift River and Fever Brook watersheds

20. Results of mineralogic analysis of clay-size (less than 2 micrometers) fraction of soil samples

21. Physical characteristics and concentrations of chemical constituents of soil waters from the West Branch Swift River and East Branch Fever Brook basins 


\section{CONVERSION FACTORS AND ABBREVIATIONS}

For the convenience of readers who may prefer to use metric (International System) units rather than the inch-pound units used in this report, values can be converted by using the following factors.

Multiply inch-pound unit

By

To obtain metric unit

\section{Length}

inch (in.)

2.54

centimeter (cm)

foot (ft)

0.3048

meter (m)

mile (mi)

1.609

kilometer (km)

square mile $\left(\mathrm{mi}^{2}\right)$

cubic foot $\left(\mathrm{ft}^{3}\right)$
cubic foot per second $\left(\mathrm{ft}^{3} / \mathrm{s}\right)$
cubic foot per second per square mile $\left[\left(\mathrm{ft}^{3} / \mathrm{s}\right) / \mathrm{mi}^{2}\right]$

\section{Area}

2.590

259.0

Volume

0.02832

Flow

0.02832

0.01093 square kilometer $\left(\mathrm{km}^{2}\right)$

hectare (ha)

cubic meter $\left(\mathrm{m}^{3}\right)$

cubic meter per second $\left(\mathrm{m}^{3} / \mathrm{s}\right)$

cubic meter per second per square kilometer $\left[\left(\mathrm{m}^{3} / \mathrm{s}\right) / \mathrm{km}^{2}\right]$

\section{Temperature}

Temperature in degrees Celsius $\left({ }^{\circ} \mathrm{C}\right)$ can be converted to degrees Fahrenheit $\left({ }^{\circ} \mathrm{F}\right)$ as follows: ${ }^{\circ} \mathrm{F}=\left(1.8 \times{ }^{\circ} \mathrm{C}\right)+32$ 


\title{
Water Quality and Hydrologic Data for the West Branch Swift River and East Branch Fever Brook, Quabbin Reservoir Drainage Basin, Massachusetts
}

\author{
By Rochelle L. Rittmaster and Gerald G. Girouard
}

\begin{abstract}
Water quality and hydrologic data were collected as part of a study of the effects of acid precipitation on stream water quality in two watersheds in central Massachusetts, conducted by the U.S. Geological Survey from November 1983 through September 1985. The two watersheds, West Branch Swift River (12.7 square miles) and East Branch Fever Brook (4.85 square miles), located north of Quabbin Reservoir, differ in surficial geology and hydrologic characteristics. Data are reported on precipitation quality and quantity and streamflow quality and quantity at the outlet of each watershed and at several tributaries. Also reported are the quality of soil water and ground water, chemistry of soil leachates, and mineralogical analyses of the clay-sized fraction of watershed soils.
\end{abstract}

\section{INTRODUCTION}

This report presents data collected by the U.S. Geological Survey from November 1983 through September 1985 during a study of effects of acid precipitation on stream-water quality. A paired watershed approach was used to assess the influence of geology and surface hydrologic characteristics on ameliorating the effects of acid deposition. The study was conducted in cooperation with the Water Pollution Control Division of the Massachusetts Department of Environmental Protection (MDEP) and the Metropolitan District Commission (MDC).
The MDC operates Quabbin Reservoir, the primary source of public water supply for the Boston metropolitan area in eastern Massachusetts.

The West Branch Swift River and East Branch Fever Brook are located in central Massachusetts to the northwest and northeast, respectively, of Quabbin Reservoir (fig. 1). The West Branch Swift River (Swift River) drains a basin of $12.7 \mathrm{mi}^{2}$ (square miles) that is characterized by steep till-covered bedrock ridges in the headwater areas and gently sloped stratified drift near the basin outlet. The East Branch Fever Brook (Fever Brook), which drains $4.85 \mathrm{mi}^{2}$, has gentle slopes with till and stratified drift mixed throughout the basin.

The data presented include chemical and hydrologic information for the determination of water yields and chemical quality of water used for supply and recreational purposes. They include chemical analyses of precipitation and stream samples collected approximately weekly, and daily mean discharge at gaging stations on Swift River and Fever Brook (figs. 2, 3). Wells drilled in each of the drainage basins were sampled approximately monthly for chemical analysis of ground water. Soil samples were analyzed for physical, chemical, and mineralogical properties, and soil-water samples were collected for chemical analysis.

Most of the data in this report are presented separately for each watershed and shown in tables at the end of this report. Hydrologic and chemical data collected from the Swift River basin are listed in tables 2 through 9. Similar data for the Fever Brook basin are presented in tables 10 through 17. Data on the soils and soil-water samples collected in both basins are combined in tables 18 through 21 . 


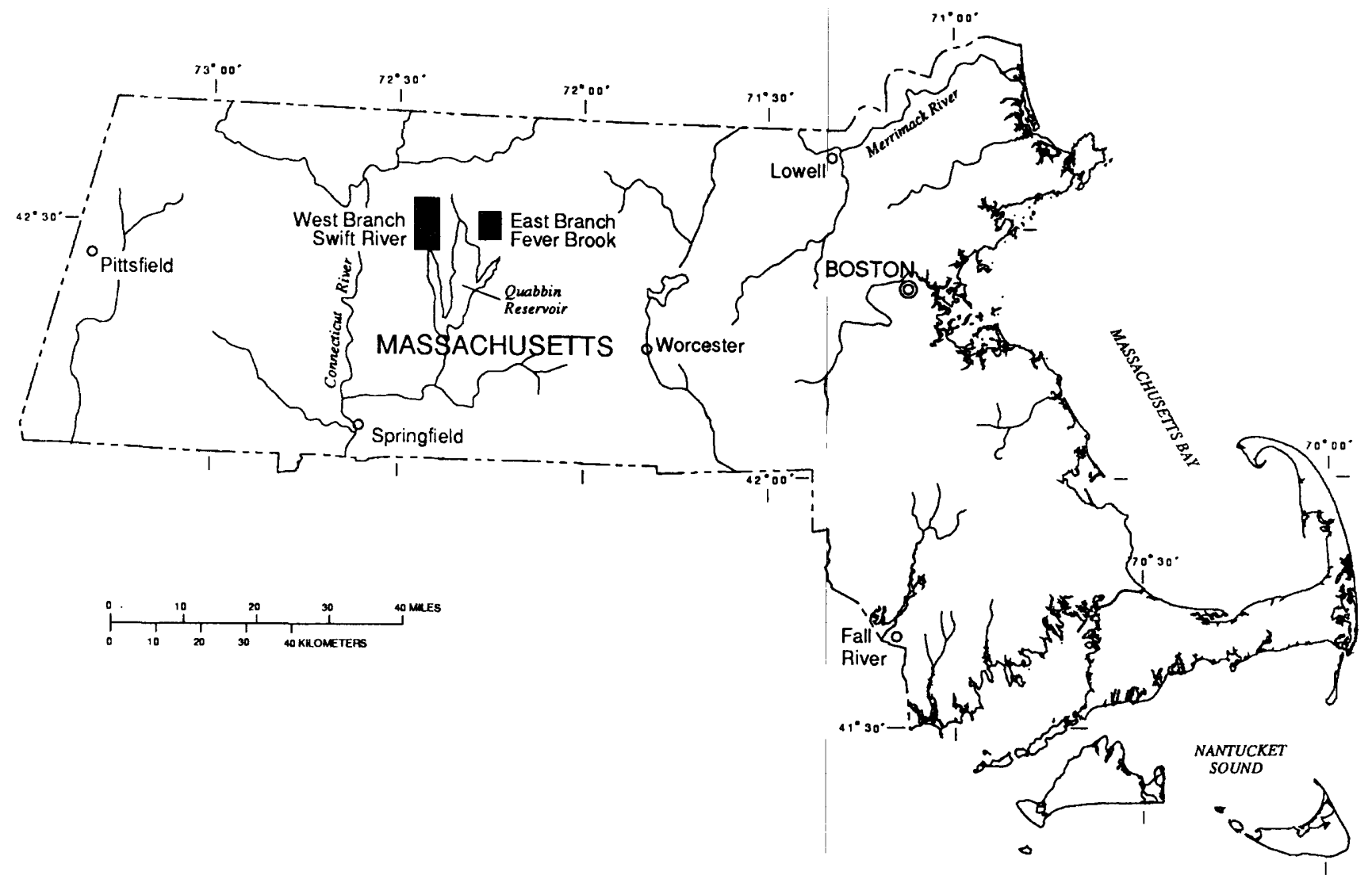

Figure 1.--Locations of the West Branch Swift River and East Branch Fever Brook drainage basins, Massachusetts.

The authors are grateful to State officials and local residents who provided time and information for this study. Special thanks are extended to Mr. David Ashenden and Mr. Bruce Spencer of the MDC for their on-site assistance and observations, and to Gail Batchelder at the University of Massachusetts at Amherst, Massachusetts, for her laboratory analyses of soil samples.

\section{SAMPLE-SITE NUMBERING SYSTEM}

A sample site numbering system for surface water and ground water was used to identify, record, organize, and track sample analyses. Eight-digit station numbers were assigned to each surface-water gaging station and surface-water sampling site. These numbers were assigned in downstream order.
Gaps left in the series of numbers reserves station numbers for assignment in proper sequence should intermediate stations be established in the future.

Ground-water and soil-water sampling sites were assigned 15-digit identification numbers. The first 13 digits provide the geographic coordinates of the site in degrees, minutes, and seconds of latitude and longitude. The last 2 digits are sequential identification numbers for sites within the 1 -second by 1 -second quadrangle identified by the 13-digit latitude-longitude.

\section{COLLECTION AND COMPUTATION OF DATA}

Wet deposition was collected weekly, using an Aerochem-Metrics ${ }^{1}$ Model 301 wet/dry deposition sampler. The sampler was operated on private

1

Use of brand names in this report is for identification purposes only and does not constitute endorsement by the U.S. Geological Survey. 
property, in an open field, on undisturbed pastureland, and was set on a platform to diminish potential for vegetation and dust contamination of the samples. Because of a change in land ownership of the original site, the collector was moved in April 1985 to a site $0.5 \mathrm{mi}$ (miles) away that was owned by the MDC. The samples were collected as 7-day composites of wet deposition. Chemical analyses of those samples are identified in table 1 by the beginning date of the 7-day period. Samples were generally collected on Tuesday to coincide with the day designated for weekly precipitation collection for the National Atmospheric Deposition Program (NADP) (Bigelow, 1982). Samples were analyzed for major cations and anions, nutrients, and some trace metals. Some weeks have only partial chemical data listed because of insufficient quantity of precipitation or malfunction or vandalism of the equipment.

Daily mean discharges for stations on the Swift River (01174565) and Fever Brook (01174050) were computed from continuous records of stage and intermittent measurements of stream discharge and stage (tables 2 and 10). These data have also been published by the U.S. Geological Survey (1987). Stage was recorded at 15 -minute intervals on a punch-tape water-stage recorder. Instantaneous discharge was measured with a Price current meter, using general guidelines of the U.S. Geological Survey (Rantz and others, 1982). The relation between stage and discharge was developed using discharge measurements made over a range of stages. Observations of factors affecting stage, such as ice, vegetation, and beaver impoundments were used to adjust stage records in determining the daily flows reported in tables 2 and 10.

Heavy rains in May 1984 resulted in stream levels above maximum stage-recording levels of the gaging stations. Peak discharge for this event was estimated using the slope-area method (Dalrymple and Benson, 1967), based on high water marks established during peak flow at the gaging stations.

Stream samples were collected at the gaging sites approximately weekly and more frequently during snowmelt and following some rainstorms (tables 3 and 11). This sampling scheme permitted evaluation of water quality on the basis of seasonality and hydrologic conditions. Water samples also were collected at tributaries to Swift River and Fever Brook during late-winter and spring snowmelt, summer low flow, and autumn-winter baseflow conditions (tables 4-7, and 11-14). Except for the gaging site on Swift River, grab samples were collected at all stream sampling locations because waters were well-mixed, as identified by measurements of uniform specific conductance, $\mathrm{pH}$, and temperature across the channels. The Swift River gaging site was located downstream of the confluence of a tributary to the main channel, and lateral profiles of $\mathrm{pH}$ and specific conductance indicated incomplete mixing of the tributary and main channel waters. At this site, samples were collected across the stream channel and composited in a churn splitter.

Monitoring wells were drilled in till and stratified drift in each drainage basin. Water levels were measured approximately monthly, and water samples were collected monthly to bimonthly (tables 8-9 and 15-17). At least three well volumes were evacuated from the wells in stratified drift prior to each sampling. Because the wells in till recovered slowly upon evacuation of standing water, sometimes only two well volumes were removed before each sampling.

Water from the unsaturated zone in both basins was sampled periodically, when near-saturated conditions existed (table 21). Two types of collectors were used: (1) zero-tension lysimeters, consisting of polyvinyl chloride (PVC) pipe cut lengthwise to form a trough, and driven into the soil, and (2) a negative-tension TIMCO lysimeter with porous Teflon cups installed at depths ranging from 4 to 18 in. (inches) below ground surface. Because water drained quickly through these soils, water was recovered only from the deep lysimeter in the Swift River basin and from the deep and medium depth lysimeters in the Fever Brook basin. Soil-water samples yielded variable amounts of water, some sufficient only for $\mathrm{pH}$ and specific conductance and some sufficient for full chemical analysis $(\mathrm{pH}$, specific conductance, major cations and anions).

Soil samples were collected from the O, A, and B horizons at several sites in the study basins (table 18). The sites were selected to provide an areal distribution and variation in surficial geology and underlying bedrock type.

\section{SAMPLING PROCEDURES AND HANDLING}

Temperature, specific conductance, and $\mathrm{pH}$ were measured in the field. An Extech digital conductivity meter and an Extech model 609 digital pH meter with an Orion glass combination $\mathrm{pH}$ electrode were used. Two reference solutions (Barnes, 1964) of low ionic strength were used to calibrate the $\mathrm{pH}$ meter prior to sample measurement. 







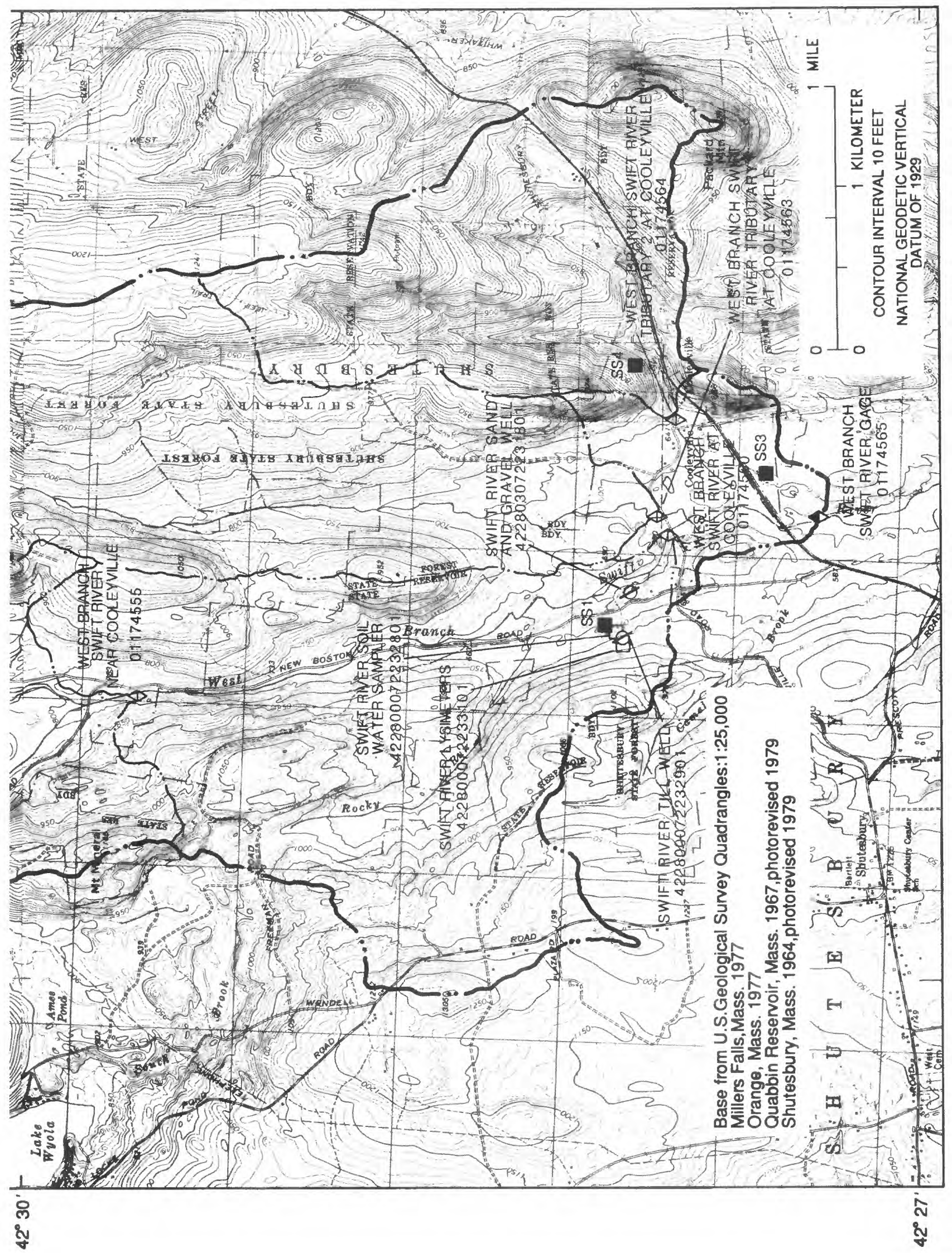

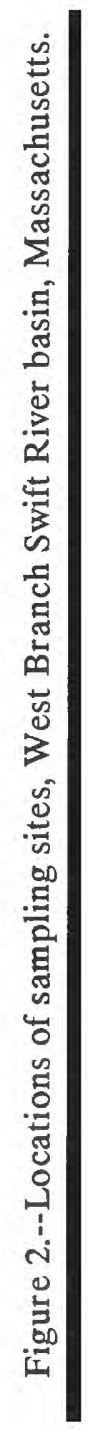




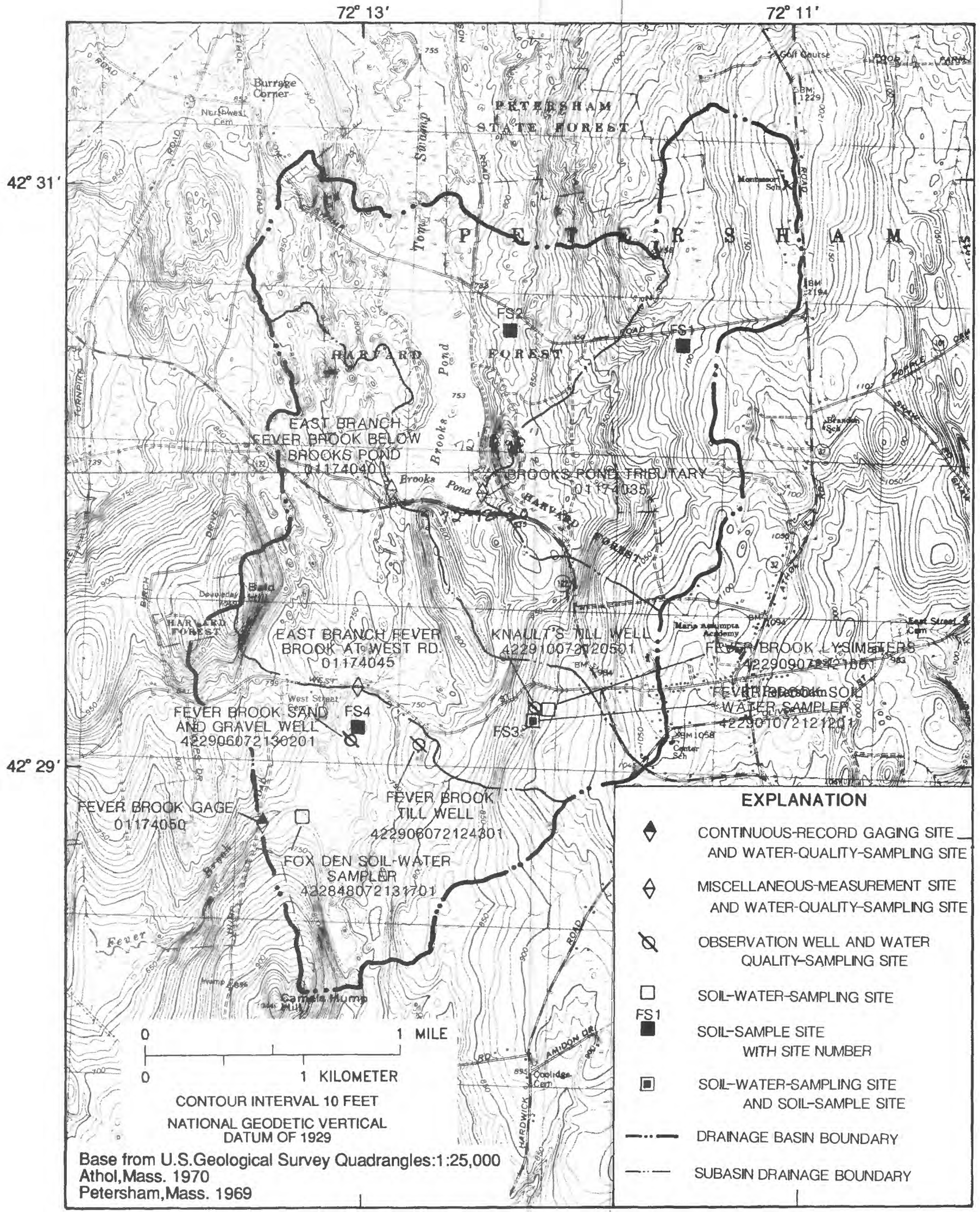

Figure 3.--Locations of sampling sites, East Branch Fever Brook basins, Massachusetts. 
Samples were filtered, preserved, and chilled in the field in accordance with methods recommended by the U.S. Geological Survey (Skougstad and others, 1979). Samples were shipped on ice to the U.S. Geological Survey Central Laboratory in Doraville, Georgia, for chemical analysis. Part of each sample was retained for measurement of alkalinity in a laboratory in the Massachusetts office.

Initially, dissolved aluminum was analyzed from samples that passed through a $0.4-\mu \mathrm{m}$ (micrometer) filter. However, investigators (Jones and others, 1974) have observed that particulate and colloidal aluminum can pass through that filter pore size, causing overestimation of monomeric, "free" aluminum concentrations, the form of aluminum considered most toxic to fish (Driscoll and others, 1980). Therefore, during the first year of sample collection, two aliquots were periodically submitted for dissolved aluminum analysis -- one having passed through a $0.4-\mu \mathrm{m}$ filter, and one having passed through a $0.1-\mu \mathrm{m}$ filter. Dissolved aluminum concentrations reported for water samples collected at the two basin outlets after December 1, 1984, are only for those samples that passed through the $0.1-\mu \mathrm{m}$ filter.

\section{LABORATORY ANALYSES}

Concentrations of chemical constituents in the water samples were determined by methods outlined by Skougstad and others (1979). Many of the methods were developed especially for the analysis of low ionic-strength waters. Alkalinity was measured by incremental titration (M.C. Yurewicz, U.S. Geological Survey, written communication, 1985) using 0.01639-molar sulfuric acid as the titrant. This method is sensitive to the detection of the actual buffering threshold of water, the point at which the $\mathrm{pH}$ drops very quickly with small additions of acid. In contrast, a fixed-endpoint titration requires acid titration of the sample to $\mathrm{pH} 4.3$ or 4.5 . Because total carbonic-acid concentrations are low in these waters, the true endpoint generally is at a $\mathrm{pH}$ significantly higher ( $\mathrm{pH} 5.0$ to 5.3) than that of a fixedendpoint titration. Therefore, the alkalinity measured by incremental titration is more conservative and accurate than the alkalinity measured by fixed-endpoint titration in the determination of the buffering capacities of the waters.

Chemical analysis of soil-water leachates included exchangeable base cations (calcium, magnesium, sodium, and potassium) and exchangeable acidity (aluminum and hydrogen) (table 19) (Thomas, 1982). Mineralogical analysis of the clay- size fraction (less than $2 \mu \mathrm{m}$ ) of watershed soils was performed at the Department of Geology and Geography, University of Massachusetts, using X-ray diffraction.

\section{REFERENCES}

Barnes, I., 1964, Field measurement of alkalinity and pH: U.S. Geological Survey Water-Supply Paper 1535-H, 17 p.

Bigelow, D.S., 1982, National Atmospheric Deposition Program instruction manual, site operation: Program Coordinator's Office, National Atmospheric Deposition Program, Colorado State University, Fort Collins, CO, $30 \mathrm{p}$.

Dalrymple, T., and Benson, M.A., 1967, Measurement of peak discharge by the slope-area method: Techniques of Water-Resources Investigations of the U.S. Geological Survey, Book 3, Chapter A2, 12 p.

Driscoll, C.J., Jr., Baker, J.P., Bisogni, J.J., Jr., and Schofield, C.L., 1980, Effect of aluminum speciation on fish in dilute acidified waters: Nature, v. 284, p. 161-164.

Jones, B.F., Kennedy, V.C., and Zellweger, G.W., 1974, Comparison of observed and calculated concentrations of dissolved aluminum and iron in stream water: Water Resources Research, v. 10, p. 791-793.

Rantz, S.E., and others, 1982, Measurement and computation of streamflow: v. 1, measurement of stage and discharge: U.S. Geological Survey Water-Supply Paper 2175, 284 p.

Skougstad, M.W., Fishman, M.J., Friedman, L.C., Erdmann, D.E., and Duncan, S.S., eds., 1979, Methods for determination of inorganic substances in water and fluvial sediments: U.S. Geological Survey Techniques of WaterResources Investigations, Book 5, Chapter A1, $626 \mathrm{p}$.

Thomas, G.W., 1982, Exchangeable cations in Page, A.L., Muller, R.H., and Keeney, D.R. (eds.), Methods of Soil Analysis, Part 2, Chemical and Biological Properties: Agronomy Monograph 9, Madison, WI., p. 159-164.

U.S. Geological Survey, 1987, Water Resources Data, Massachusetts and Rhode Island, Water Year 1985, U.S. Geological Survey Water-Data Report MA-RI-85-1. 


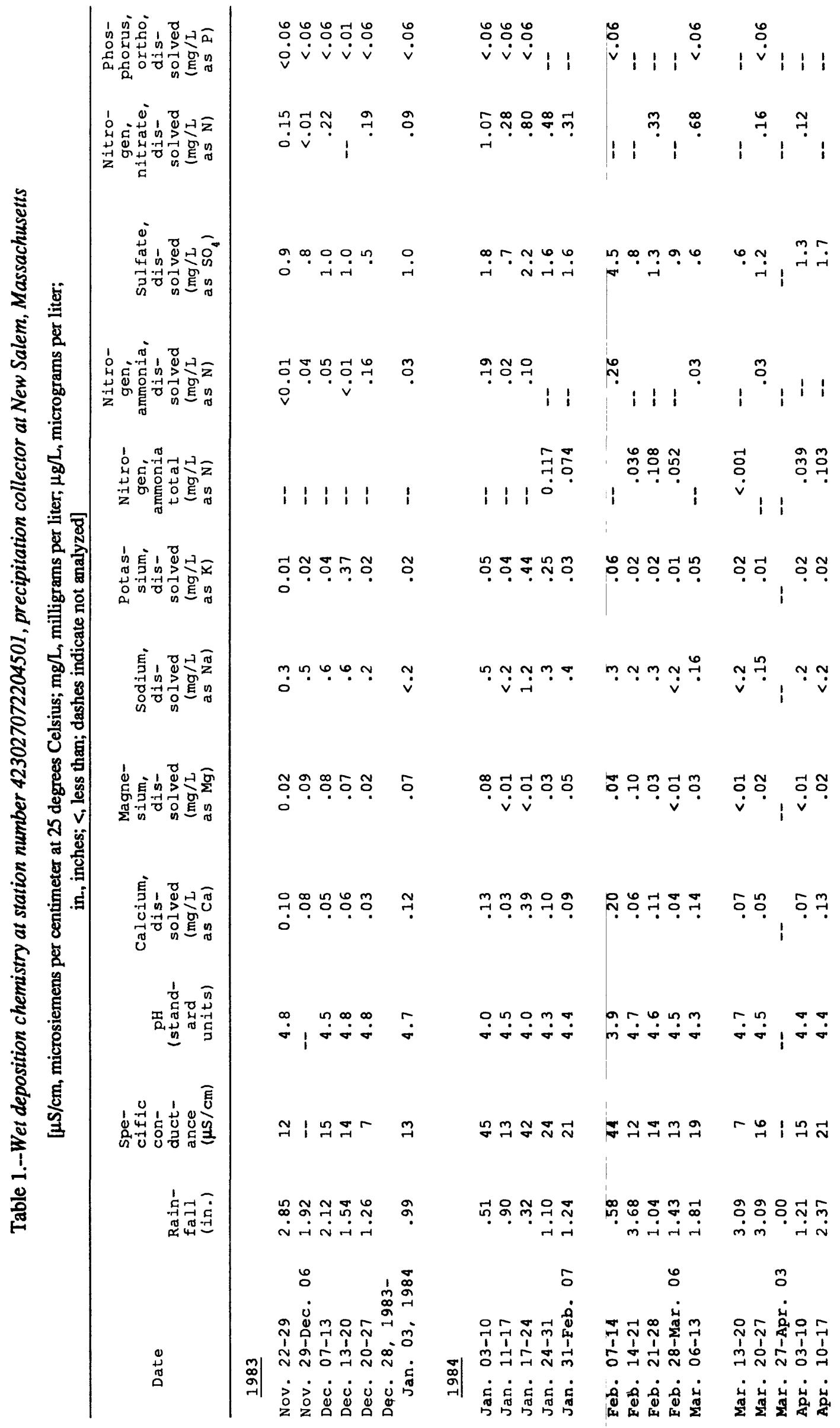




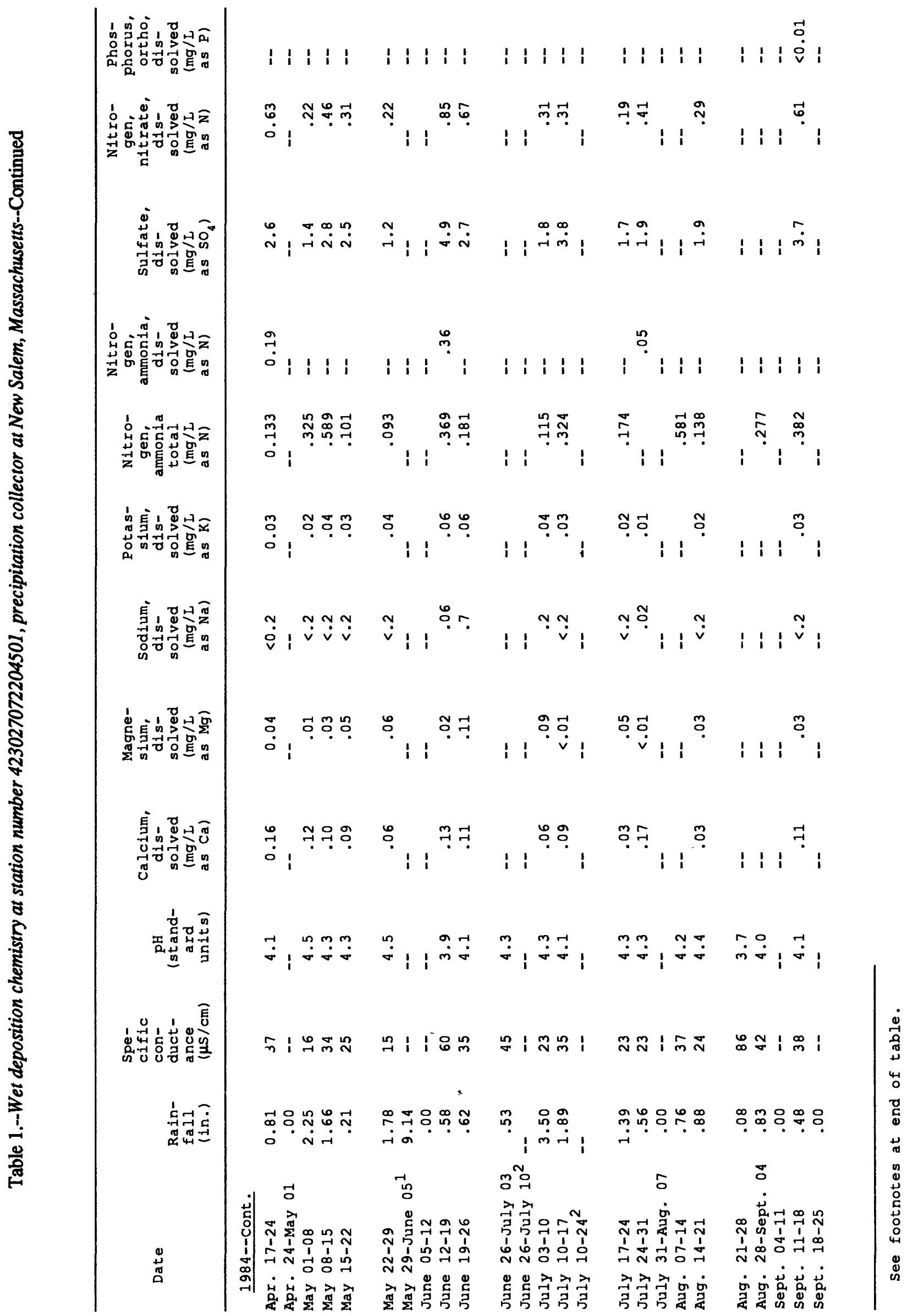




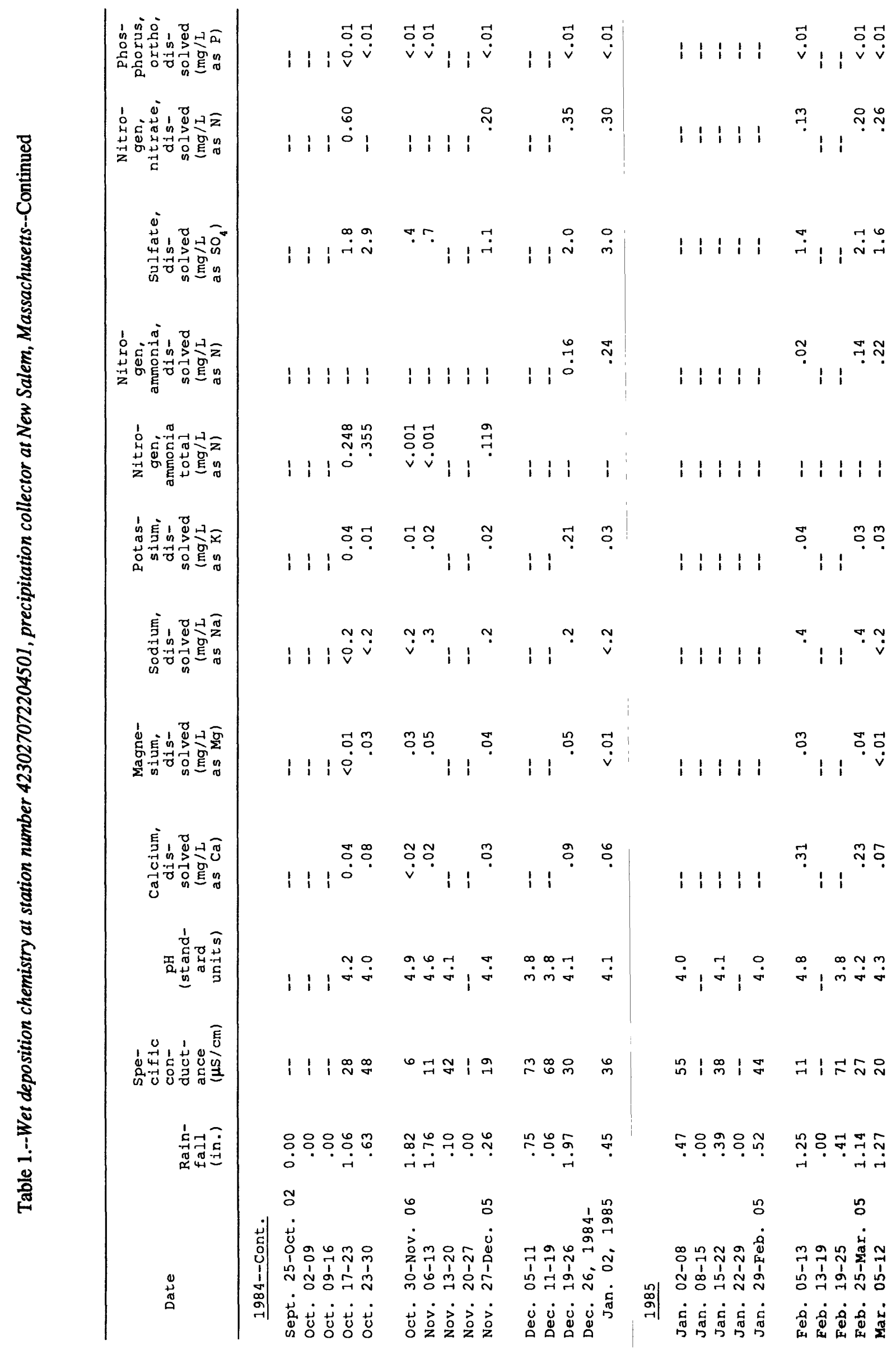




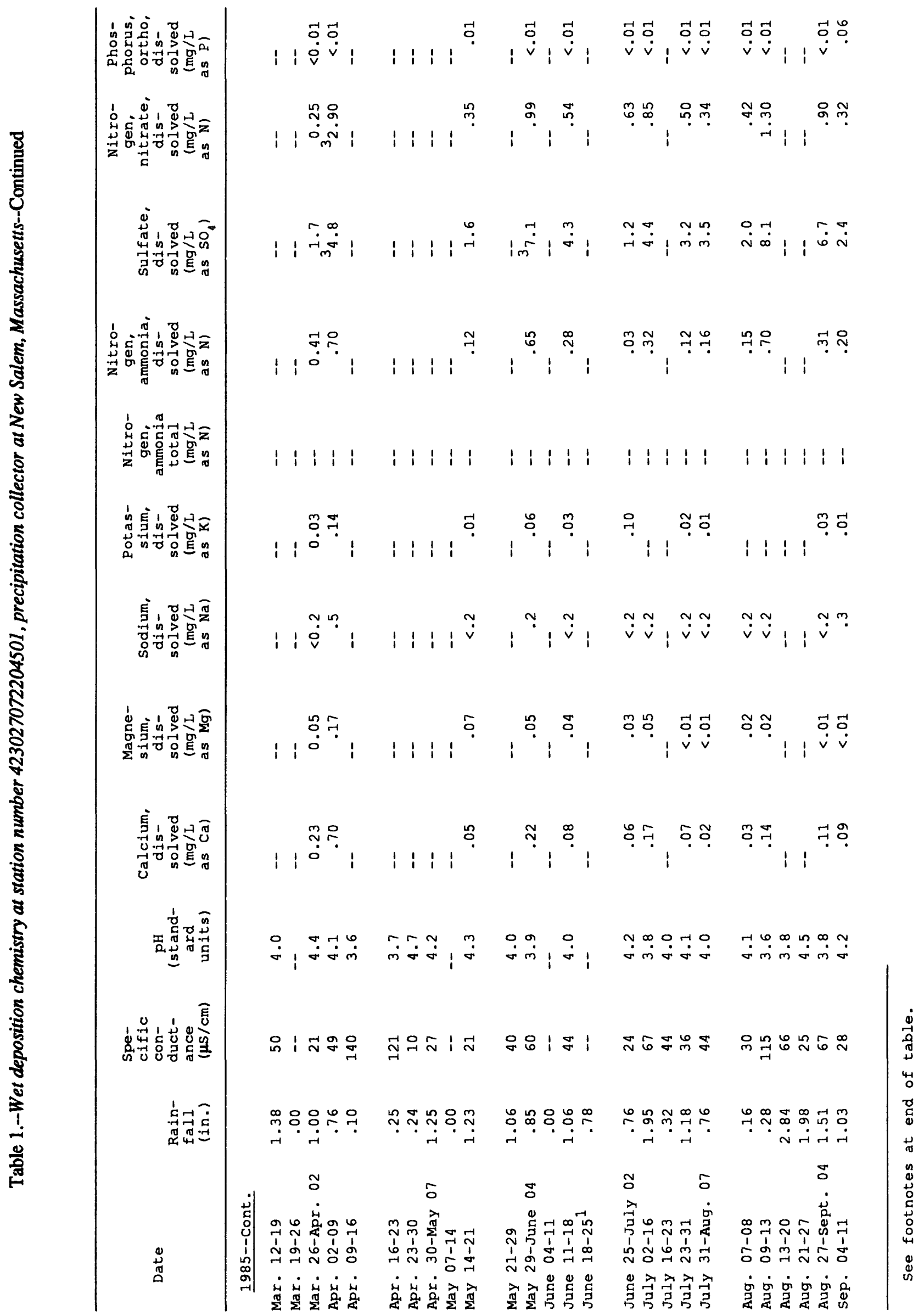




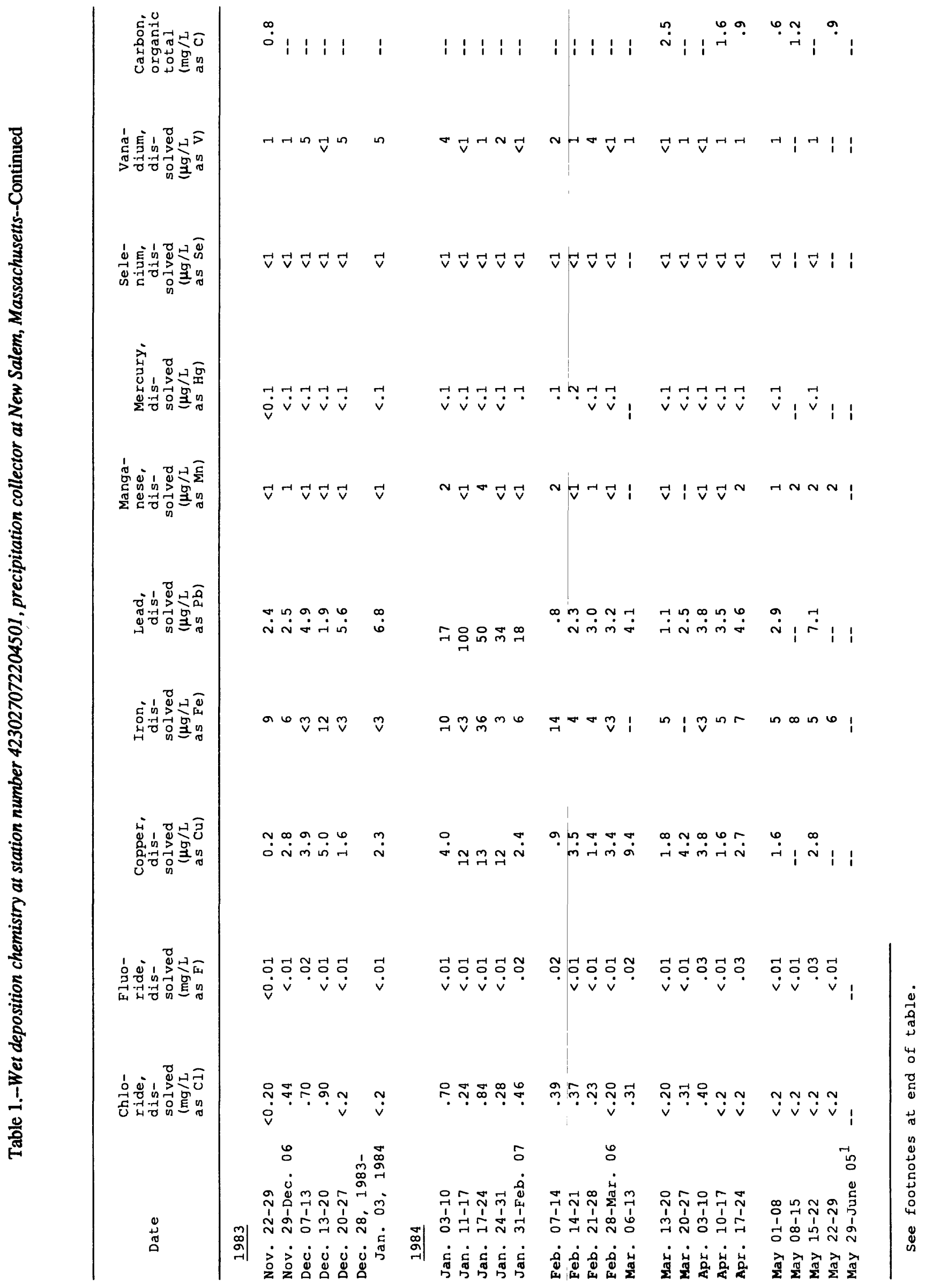




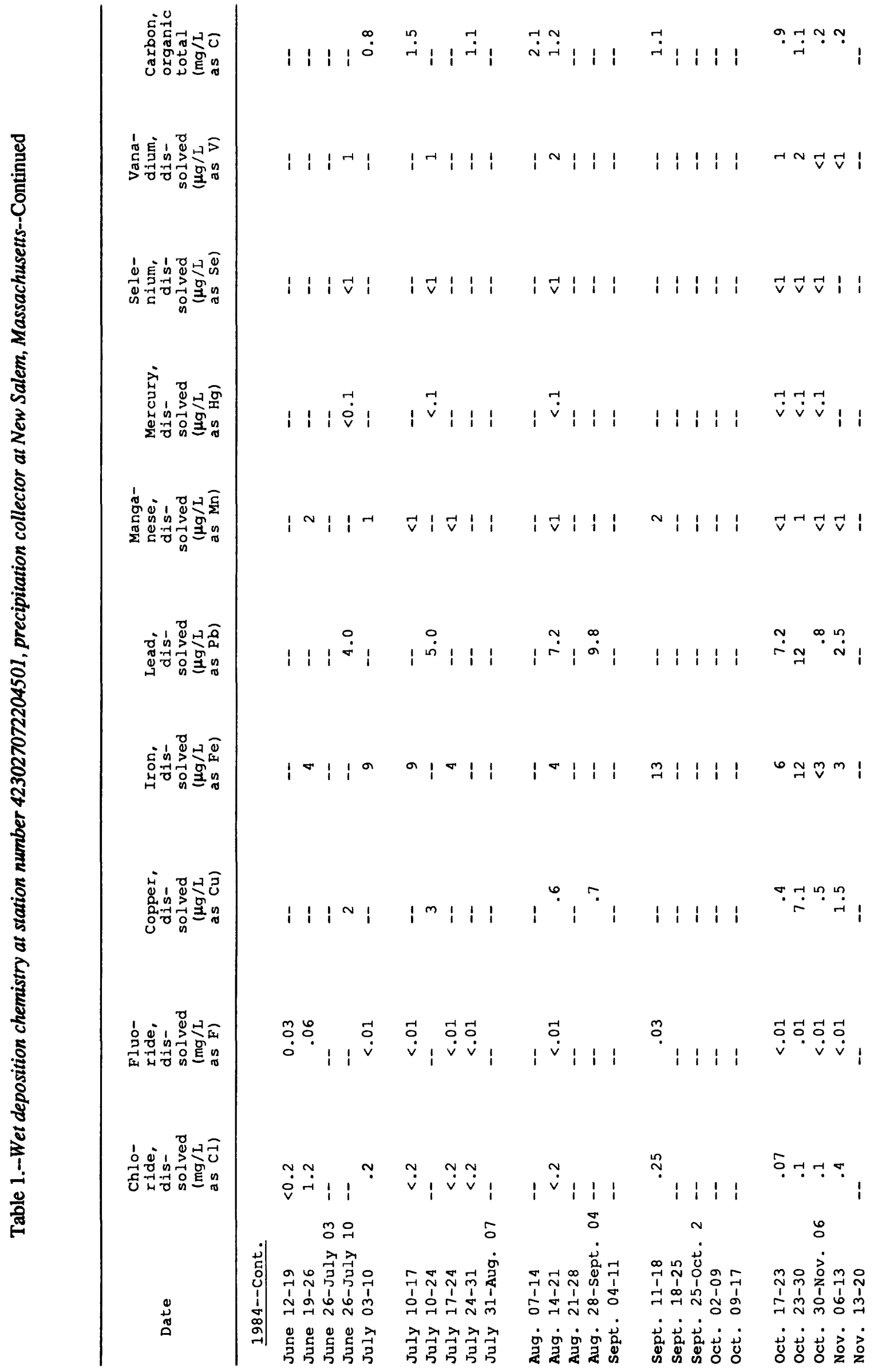




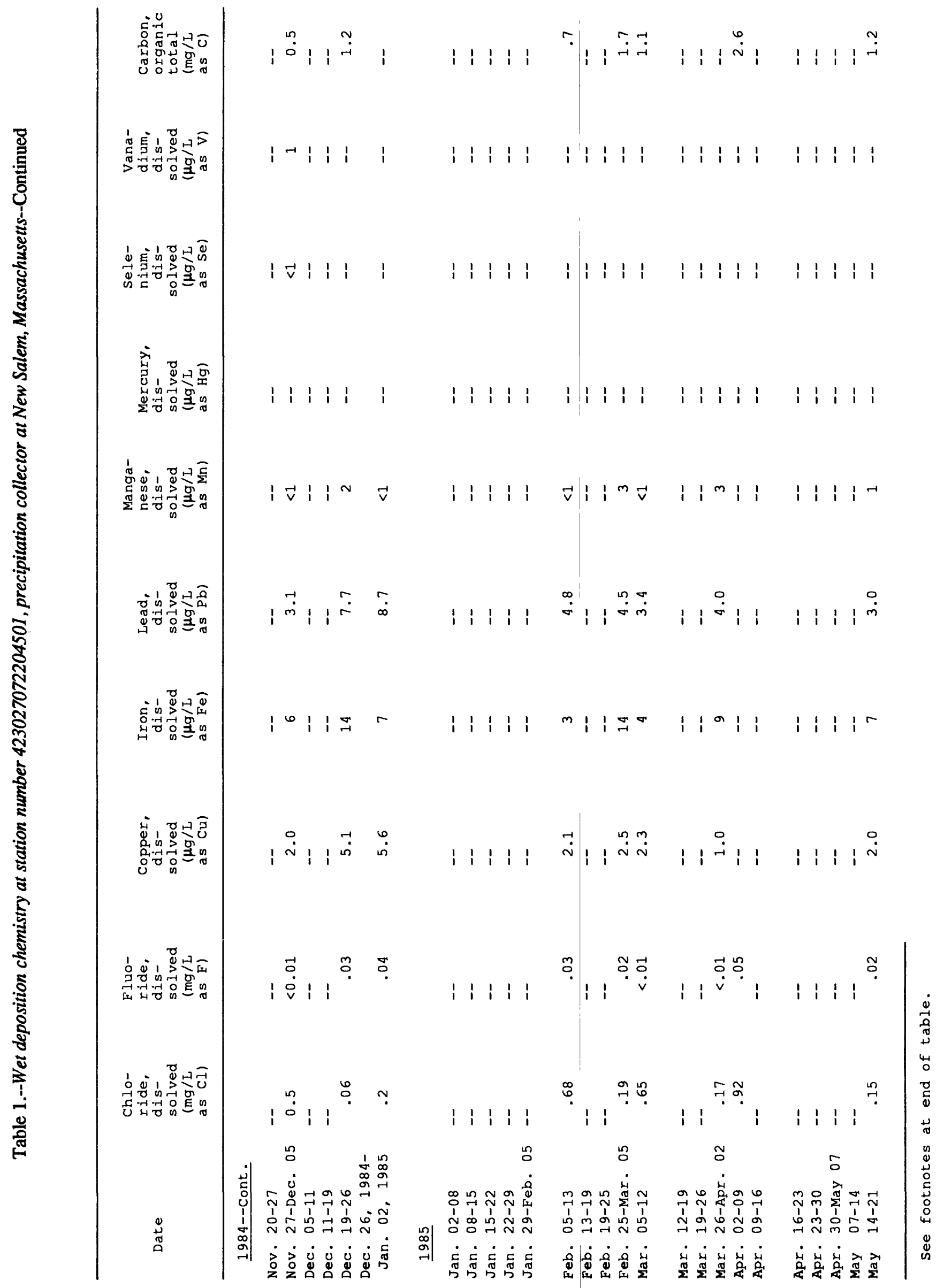




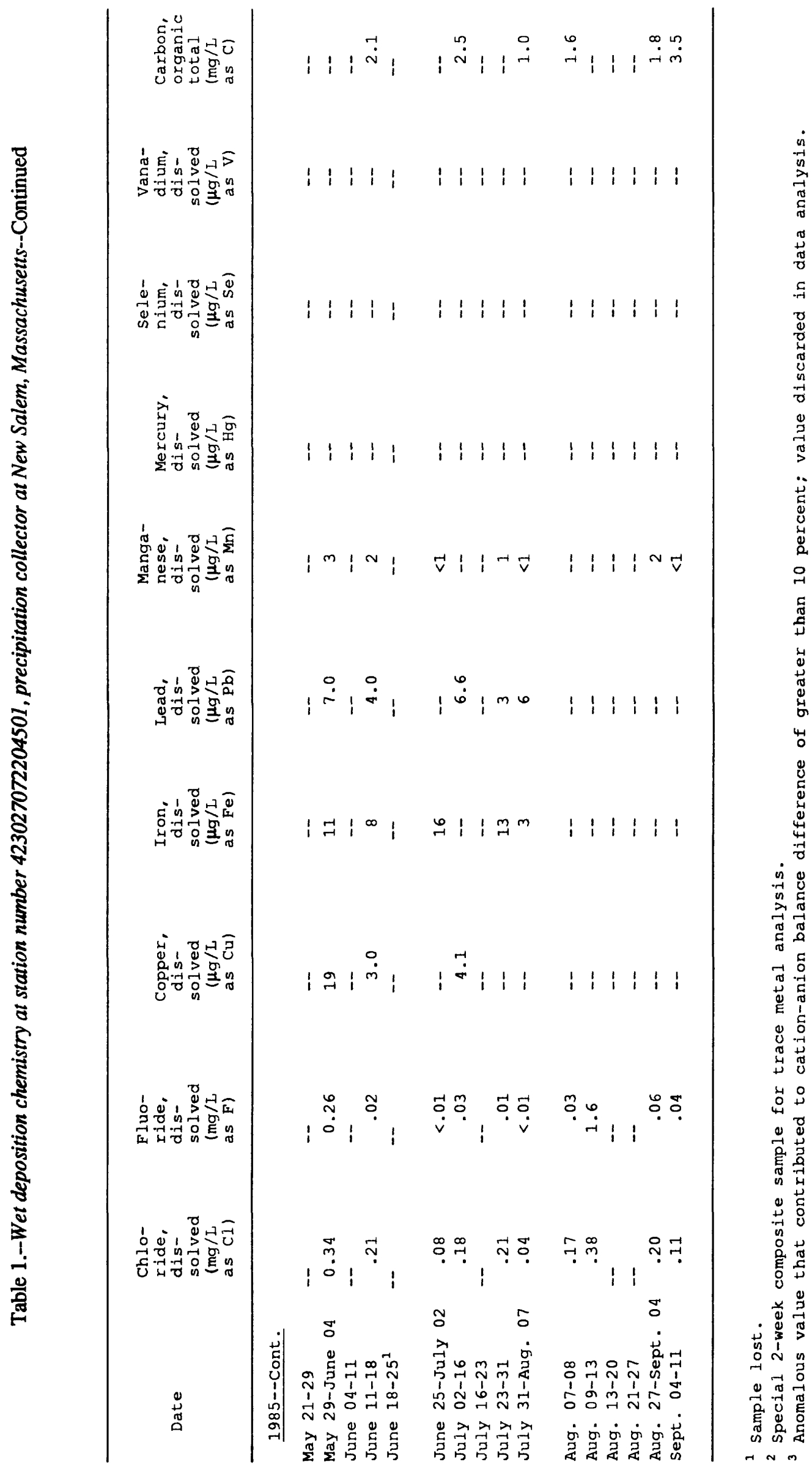


Table 2.--Daily mean discharge at station number 01174565, West Branch Swift River near Shutesbury, Massachusetts

[Period of record begins November 8,$1983 ;\left(\mathrm{ft}^{3} / \mathrm{s}\right) / \mathrm{mi}^{2}$, cubic feet per second per square mile; dashes indicate not applicable]

Discharge, in cubic feet per second, water year bctober 1983 to September 1984

\begin{tabular}{|c|c|c|c|c|c|c|c|c|c|c|c|c|}
\hline Day & Oct & Nov & Dec & Jan & Feb & Mar & Apr & May & June & July & Aug & Sept \\
\hline $\begin{array}{l}1 \\
2 \\
3 \\
4 \\
5\end{array}$ & $\begin{array}{l}-- \\
-- \\
-- \\
--\end{array}$ & $\begin{array}{l}-- \\
-- \\
-- \\
-- \\
--\end{array}$ & $\begin{array}{l}35 \\
29 \\
25 \\
24 \\
24\end{array}$ & $\begin{array}{l}34 \\
29 \\
27 \\
26 \\
24\end{array}$ & $\begin{array}{l}15 \\
15 \\
19 \\
39 \\
44\end{array}$ & $\begin{array}{l}51 \\
43 \\
37 \\
35 \\
32\end{array}$ & $\begin{array}{r}40 \\
48 \\
57 \\
65 \\
238\end{array}$ & $\begin{array}{r}26 \\
24 \\
24 \\
100 \\
60\end{array}$ & $\begin{array}{r}205 \\
159 \\
114 \\
78 \\
61\end{array}$ & $\begin{array}{l}7.4 \\
7.6 \\
7.2 \\
6.5 \\
5.6\end{array}$ & $\begin{array}{l}4.3 \\
3.9 \\
3.8 \\
3.5 \\
3.2\end{array}$ & $\begin{array}{l}2.1 \\
1.7 \\
1.9 \\
2.6 \\
2.8\end{array}$ \\
\hline $\begin{array}{r}6 \\
7 \\
8 \\
9 \\
10\end{array}$ & $\begin{array}{l}-- \\
-- \\
-- \\
--\end{array}$ & $\begin{array}{l}-- \\
-- \\
3.6 \\
3.8 \\
4.2\end{array}$ & $\begin{array}{r}26 \\
118 \\
59 \\
39 \\
34\end{array}$ & $\begin{array}{l}24 \\
24 \\
22 \\
21 \\
20\end{array}$ & $\begin{array}{l}38 \\
28 \\
22 \\
21 \\
20\end{array}$ & $\begin{array}{l}36 \\
35 \\
30 \\
29 \\
27\end{array}$ & $\begin{array}{r}353 \\
172 \\
121 \\
92 \\
77\end{array}$ & $\begin{array}{l}40 \\
35 \\
45 \\
70 \\
45\end{array}$ & $\begin{array}{l}50 \\
44 \\
38 \\
32 \\
27\end{array}$ & $\begin{array}{r}18 \\
164 \\
67 \\
29 \\
20\end{array}$ & $\begin{array}{l}2.8 \\
3.6 \\
5.6 \\
3.6 \\
3.3\end{array}$ & $\begin{array}{l}1.9 \\
1.4 \\
1.4 \\
1.4 \\
1.3\end{array}$ \\
\hline $\begin{array}{l}11 \\
12 \\
13 \\
14 \\
15\end{array}$ & $\begin{array}{l}-- \\
-- \\
-- \\
--\end{array}$ & $\begin{array}{r}23 \\
19 \\
12 \\
9.4 \\
8.5\end{array}$ & $\begin{array}{r}31 \\
29 \\
175 \\
299 \\
126\end{array}$ & $\begin{array}{l}21 \\
20 \\
20 \\
19 \\
18\end{array}$ & $\begin{array}{l}20 \\
25 \\
27 \\
29 \\
81\end{array}$ & $\begin{array}{l}25 \\
23 \\
21 \\
21 \\
24\end{array}$ & $\begin{array}{l}65 \\
65 \\
63 \\
68 \\
92\end{array}$ & $\begin{array}{l}35 \\
40 \\
60 \\
90 \\
60\end{array}$ & $\begin{array}{l}23 \\
20 \\
18 \\
18 \\
17\end{array}$ & $\begin{array}{r}15 \\
14 \\
11 \\
8.2 \\
6.9\end{array}$ & $\begin{array}{l}2.9 \\
2.3 \\
2.2 \\
2.2 \\
2.2\end{array}$ & $\begin{array}{l}1.2 \\
1.0 \\
1.0 \\
1.0 \\
1.2\end{array}$ \\
\hline $\begin{array}{l}16 \\
17 \\
18 \\
19 \\
20\end{array}$ & $\begin{array}{l}-- \\
-- \\
-- \\
--\end{array}$ & $\begin{array}{l}68 \\
55 \\
26 \\
19 \\
15\end{array}$ & $\begin{array}{l}83 \\
63 \\
52 \\
46 \\
38\end{array}$ & $\begin{array}{l}17 \\
15 \\
14 \\
14 \\
13\end{array}$ & $\begin{array}{l}405 \\
143 \\
184 \\
121 \\
112\end{array}$ & $\begin{array}{l}24 \\
29 \\
29 \\
31 \\
36\end{array}$ & $\begin{array}{r}131 \\
110 \\
73 \\
70 \\
65\end{array}$ & $\begin{array}{l}43 \\
37 \\
32 \\
29 \\
26\end{array}$ & $\begin{array}{l}15 \\
14 \\
15 \\
17 \\
14\end{array}$ & $\begin{array}{l}12 \\
18 \\
25 \\
24 \\
14\end{array}$ & $\begin{array}{r}1.7 \\
1.6 \\
1.6 \\
1.5 \\
10\end{array}$ & $\begin{array}{l}1.4 \\
1.4 \\
1.4 \\
1.1 \\
1.0\end{array}$ \\
\hline $\begin{array}{l}21 \\
22 \\
23 \\
24 \\
25\end{array}$ & $\begin{array}{l}-- \\
-- \\
-- \\
-- \\
--\end{array}$ & $\begin{array}{r}40 \\
37 \\
26 \\
25 \\
144\end{array}$ & $\begin{array}{l}35 \\
37 \\
40 \\
35 \\
30\end{array}$ & $\begin{array}{l}14 \\
14 \\
14 \\
17 \\
46\end{array}$ & $\begin{array}{l}85 \\
64 \\
54 \\
87 \\
86\end{array}$ & $\begin{array}{l}42 \\
69 \\
70 \\
54 \\
47\end{array}$ & $\begin{array}{l}62 \\
51 \\
45 \\
51 \\
50\end{array}$ & $\begin{array}{l}25 \\
22 \\
24 \\
36 \\
26\end{array}$ & $\begin{array}{l}13 \\
11 \\
11 \\
10 \\
12\end{array}$ & $\begin{array}{l}10 \\
9.2 \\
7.4 \\
6.7 \\
5.9\end{array}$ & $\begin{array}{l}4.0 \\
3.2 \\
2.4 \\
2.4 \\
2.1\end{array}$ & $\begin{array}{r}1.0 \\
.90 \\
.90 \\
.90 \\
.90\end{array}$ \\
\hline $\begin{array}{l}26 \\
27 \\
28 \\
29 \\
30 \\
31\end{array}$ & $\begin{array}{l}-- \\
-- \\
-- \\
-- \\
-- \\
--\end{array}$ & $\begin{array}{r}130 \\
73 \\
54 \\
62 \\
49 \\
--\end{array}$ & $\begin{array}{l}27 \\
25 \\
34 \\
62 \\
45 \\
37\end{array}$ & $\begin{array}{l}36 \\
24 \\
20 \\
19 \\
17 \\
17\end{array}$ & $\begin{array}{l}83 \\
61 \\
57 \\
63 \\
-- \\
--\end{array}$ & $\begin{array}{l}44 \\
43 \\
41 \\
42 \\
44 \\
38\end{array}$ & $\begin{array}{l}43 \\
36 \\
32 \\
29 \\
27 \\
--\end{array}$ & $\begin{array}{r}22 \\
20 \\
19 \\
173 \\
612 \\
521\end{array}$ & $\begin{array}{r}11 \\
9.2 \\
7.7 \\
7.6 \\
6.8 \\
--\end{array}$ & $\begin{array}{l}4.5 \\
5.5 \\
8.8 \\
6.8 \\
6.3 \\
4.9\end{array}$ & $\begin{array}{l}1.7 \\
1.4 \\
1.4 \\
1.4 \\
1.4 \\
1.8\end{array}$ & $\begin{array}{r}.90 \\
.90 \\
.90 \\
.90 \\
.90\end{array}$ \\
\hline $\begin{array}{l}\text { Tota } 1 \\
\text { Mean } \\
\text { Maximum } \\
\text { Minimum } \\
\text { (ft } / \mathrm{s} \text { ) } / \mathrm{mi}^{2} \\
\text { Inches }\end{array}$ & $\begin{array}{l}-- \\
-- \\
-- \\
2- \\
--\end{array}$ & $\begin{array}{l}-- \\
-- \\
-- \\
-- \\
-- \\
--\end{array}$ & $\begin{array}{r}1,762 \\
56.8 \\
299 \\
24 \\
4.48 \\
5.16\end{array}$ & $\begin{array}{l}660 \\
21.3 \\
46 \\
13 \\
1.68 \\
1.93\end{array}$ & $\begin{array}{c}2,048 \\
70.6 \\
405 \\
15 \\
8 \\
3 \quad 5.56 \\
6.00\end{array}$ & $\begin{array}{c}1,152 \\
37.2^{2} \\
70 \\
21 \\
2.93 \\
3.37\end{array}$ & $\begin{array}{c}2.491 \\
83.0^{2} \\
353 \\
27 \\
3 \\
7 \\
7.5 .54 \\
7.30\end{array}$ & $\begin{array}{l}2.421{ }^{1} \\
78.1 \\
612 \\
\\
19 \\
4 \\
0 \\
0.15 \\
7.09\end{array}$ & $\begin{array}{c}1,078.3 \\
35.9 \\
205 \\
\\
6.8 \\
5 \\
2.83 \\
9 \\
3.16\end{array}$ & $\begin{array}{c}556.4 \\
17.9 \\
164 \\
4.5 \\
1.41 \\
1.63\end{array}$ & $\begin{array}{c}89.0 \\
2.87 \\
10 \\
1.4 \\
.23 \\
.26\end{array}$ & $\begin{array}{c}39.30 \\
1.31 \\
2.8 \\
.90 \\
.10 \\
.12\end{array}$ \\
\hline
\end{tabular}


Table 2.--Daily mean discharge at station number 01174565, West Branch Swift River near Shutesbury, Massachusetts--Continued

Discharge, in cubic feet per second, water year October 1984 to September 1985

\begin{tabular}{|c|c|c|c|c|c|c|c|c|c|c|c|c|}
\hline Day & Oct & Nov & Dec & Jan & Feb & Mar & Apr & May & June & July & Aug & Sept \\
\hline $\begin{array}{l}1 \\
2 \\
3 \\
4 \\
5\end{array}$ & $\begin{array}{l}0.94 \\
7.6 \\
4.6 \\
3.1 \\
2.7\end{array}$ & $\begin{array}{r}2.5 \\
2.3 \\
2.1 \\
2.0 \\
32\end{array}$ & $\begin{array}{l}7.9 \\
7.2 \\
6.8 \\
8.8 \\
7.5\end{array}$ & $\begin{array}{l}20 \\
31 \\
27 \\
21 \\
17\end{array}$ & $\begin{array}{l}6.3 \\
6.2 \\
6.1 \\
6.0 \\
6.0\end{array}$ & $\begin{array}{l}18 \\
17 \\
14 \\
13 \\
17\end{array}$ & $\begin{array}{l}22 \\
22 \\
22 \\
23 \\
26\end{array}$ & $\begin{array}{l}6.9 \\
6.6 \\
13 \\
15 \\
11\end{array}$ & $\begin{array}{l}9.9 \\
8.1 \\
6.9 \\
6.0 \\
6.0\end{array}$ & $\begin{array}{l}6.9 \\
4.4 \\
3.3 \\
2.9 \\
2.6\end{array}$ & $\begin{array}{r}22 \\
12 \\
7.9 \\
5.5 \\
3.8\end{array}$ & $\begin{array}{r}13 \\
9.2 \\
7.3 \\
5.9 \\
5.1\end{array}$ \\
\hline $\begin{array}{r}6 \\
7 \\
8 \\
9 \\
10\end{array}$ & $\begin{array}{l}1.9 \\
1.9 \\
1.6 \\
1.6 \\
1.6\end{array}$ & $\begin{array}{l}27 \\
12 \\
7.7 \\
5.8 \\
6.3\end{array}$ & $\begin{array}{l}40 \\
17 \\
8.4 \\
6.7 \\
5.9\end{array}$ & $\begin{array}{l}15 \\
13 \\
12 \\
11 \\
11\end{array}$ & $\begin{array}{l}6.0 \\
6.0 \\
6.0 \\
6.0 \\
6.0\end{array}$ & $\begin{array}{l}14 \\
13 \\
14 \\
17 \\
17\end{array}$ & $\begin{array}{l}26 \\
22 \\
24 \\
23 \\
20\end{array}$ & $\begin{array}{c}12 \\
12 \\
10 \\
9.3 \\
8.8\end{array}$ & $\begin{array}{l}7.1 \\
5.8 \\
5.7 \\
6.6 \\
5.1\end{array}$ & $\begin{array}{l}2.8 \\
3.4 \\
2.9 \\
2.8 \\
2.4\end{array}$ & $\begin{array}{l}2.9 \\
2.5 \\
6.0 \\
4.2 \\
3.0\end{array}$ & $\begin{array}{l}7.6 \\
20 \\
14 \\
11 \\
22\end{array}$ \\
\hline $\begin{array}{l}11 \\
12 \\
13 \\
14 \\
15\end{array}$ & $\begin{array}{l}1.5 \\
1.5 \\
1.5 \\
1.4 \\
1.4\end{array}$ & $\begin{array}{l}9.9 \\
41 \\
25 \\
16 \\
12\end{array}$ & $\begin{array}{l}6.2 \\
7.4 \\
8.8 \\
12 \\
12\end{array}$ & $\begin{array}{l}10 \\
9.8 \\
9.4 \\
9.0 \\
8.8\end{array}$ & $\begin{array}{l}6.0 \\
6.2 \\
23 \\
25 \\
21\end{array}$ & $\begin{array}{r}18 \\
162 \\
106 \\
59 \\
45\end{array}$ & $\begin{array}{l}18 \\
17 \\
16 \\
14 \\
14\end{array}$ & $\begin{array}{l}8.2 \\
7.5 \\
7.2 \\
6.5 \\
5.8\end{array}$ & $\begin{array}{l}4.1 \\
6.2 \\
6.1 \\
5.4 \\
5.1\end{array}$ & $\begin{array}{l}2.4 \\
2.4 \\
4.3 \\
2.7 \\
2.6\end{array}$ & $\begin{array}{l}2.9 \\
2.8 \\
1.5 \\
1.3 \\
2.7\end{array}$ & $\begin{array}{r}17 \\
12 \\
8.9 \\
8.2 \\
5.6\end{array}$ \\
\hline $\begin{array}{l}16 \\
17 \\
18 \\
19 \\
20\end{array}$ & $\begin{array}{l}1.4 \\
1.3 \\
1.4 \\
1.4 \\
1.4\end{array}$ & $\begin{array}{r}12 \\
9.7 \\
8.1 \\
6.6 \\
6.0\end{array}$ & $\begin{array}{l}11 \\
11 \\
15 \\
16 \\
22\end{array}$ & $\begin{array}{l}8.6 \\
8.4 \\
8.2 \\
8.0 \\
8.0\end{array}$ & $\begin{array}{c}17 \\
15 \\
13 \\
10 \\
9.6\end{array}$ & $\begin{array}{l}37 \\
38 \\
33 \\
30 \\
29\end{array}$ & $\begin{array}{l}14 \\
13 \\
13 \\
12 \\
11\end{array}$ & $\begin{array}{l}5.9 \\
5.4 \\
18 \\
21 \\
15\end{array}$ & $\begin{array}{l}5.5 \\
8.9 \\
8.5 \\
7.2 \\
5.7\end{array}$ & $\begin{array}{l}3.1 \\
4.0 \\
2.5 \\
1.6 \\
1.3\end{array}$ & $\begin{array}{l}28 \\
12 \\
6.4 \\
5.4 \\
5.4\end{array}$ & $\begin{array}{l}5.1 \\
4.7 \\
4.2 \\
3.7 \\
3.4\end{array}$ \\
\hline $\begin{array}{l}21 \\
22 \\
23 \\
24 \\
25\end{array}$ & $\begin{array}{l}1.4 \\
1.6 \\
6.0 \\
3.9 \\
3.3\end{array}$ & $\begin{array}{l}5.7 \\
5.6 \\
5.7 \\
5.5 \\
5.1\end{array}$ & $\begin{array}{l}17 \\
26 \\
28 \\
20 \\
20\end{array}$ & $\begin{array}{l}8.0 \\
7.8 \\
7.6 \\
7.4 \\
7.2\end{array}$ & $\begin{array}{l}9.3 \\
9.4 \\
13 \\
22 \\
40\end{array}$ & $\begin{array}{l}29 \\
26 \\
23 \\
22 \\
21\end{array}$ & $\begin{array}{l}11 \\
10 \\
9.6 \\
8.6 \\
8.9\end{array}$ & $\begin{array}{l}16 \\
21 \\
13 \\
9.8 \\
7.6\end{array}$ & $\begin{array}{l}4.7 \\
4.4 \\
4.6 \\
5.6 \\
5.7\end{array}$ & $\begin{array}{l}1.2 \\
1.3 \\
1.1 \\
1.1 \\
1.4\end{array}$ & $\begin{array}{r}4.1 \\
3.3 \\
2.9 \\
2.4 \\
12\end{array}$ & $\begin{array}{l}3.1 \\
2.9 \\
2.8 \\
3.5 \\
5.0\end{array}$ \\
\hline $\begin{array}{l}26 \\
27 \\
28 \\
29 \\
30 \\
31\end{array}$ & $\begin{array}{l}4.2 \\
4.2 \\
3.6 \\
3.6 \\
3.3 \\
3.0\end{array}$ & $\begin{array}{r}5.1 \\
4.8 \\
4.7 \\
8.7 \\
11 \\
--\end{array}$ & $\begin{array}{l}19 \\
17 \\
15 \\
15 \\
25 \\
22\end{array}$ & $\begin{array}{l}7.1 \\
7.0 \\
6.8 \\
6.7 \\
6.5 \\
6.4\end{array}$ & $\begin{array}{l}32 \\
27 \\
21 \\
-- \\
-- \\
--\end{array}$ & $\begin{array}{l}19 \\
19 \\
19 \\
20 \\
19 \\
19\end{array}$ & $\begin{array}{r}8.2 \\
7.7 \\
7.9 \\
7.7 \\
7.4 \\
--\end{array}$ & $\begin{array}{r}6.8 \\
6.2 \\
9.9 \\
13 \\
9.2 \\
7.4\end{array}$ & $\begin{array}{r}5.0 \\
4.8 \\
6.4 \\
9.7 \\
9.4 \\
--\end{array}$ & $\begin{array}{l}3.5 \\
6.6 \\
3.5 \\
2.8 \\
2.5 \\
4.2\end{array}$ & $\begin{array}{r}21 \\
14 \\
9.0 \\
6.4 \\
7.8 \\
18\end{array}$ & $\begin{array}{l}4.1 \\
21 \\
33 \\
15 \\
9.3 \\
--\end{array}$ \\
\hline $\begin{array}{l}\text { Total } \\
\text { Mean } \\
\text { Maximum } \\
\text { Minimum } \\
\text { (ft } / \mathrm{s} \text { ) } / \mathrm{mi}^{2} \\
\text { Inches }\end{array}$ & $\begin{array}{c}79.84 \\
2.58 \\
7.6 \\
.94 \\
.20 \\
.23\end{array}$ & $\begin{array}{c}307.9 \\
10.3 \\
41 \\
2.0 \\
.81 \\
.90\end{array}$ & $\begin{array}{c}461.6 \\
14.9 \\
40 \\
5.9 \\
1.17 \\
1.35\end{array}$ & $\begin{array}{c}344.7 \\
11.1 \\
31 \\
6.4 \\
.88 \\
1.01\end{array}$ & $\begin{array}{c}380.1 \\
13.6 \\
40 \\
6.0 \\
1.07 \\
1.11\end{array}$ & $\begin{array}{l}947 \\
30.5 \\
162 \\
13 \\
2.41 \\
2.77\end{array}$ & $\begin{array}{c}459.0 \\
15.3 \\
26 \\
7.4 \\
1.20 \\
1.34\end{array}$ & $\begin{array}{c}325.0 \\
10.5 \\
21 \\
5.4 \\
.83 \\
.95\end{array}$ & $\begin{array}{c}190.2 \\
6.34 \\
9.9 \\
4.1 \\
.50 \\
.56\end{array}$ & $\begin{array}{l}90.5 \\
2.92 \\
6.9 \\
1.1 \\
.23 \\
.27\end{array}$ & $\begin{array}{c}239.1 \\
7.71 \\
28 \\
1.3 \\
.61 \\
.70\end{array}$ & $\begin{array}{r}287.6 \\
9.59 \\
33 \\
2.8 \\
.75 \\
.84\end{array}$ \\
\hline \multicolumn{2}{|c|}{$\begin{array}{l}\text { Calendar year } 1984 \\
\text { Water year } 1985\end{array}$} & $\begin{array}{l}\text { Total } 1 \\
\text { Total }\end{array}$ & $\begin{array}{r}11,384.21 \\
4,112.51\end{array}$ & $\begin{array}{l}\text { Mean } 31.1 \\
\text { Mean } 11.3\end{array}$ & $\begin{array}{l}\text { Maximum } \\
\text { Maximum }\end{array}$ & $\begin{array}{l}612 \\
n \\
162\end{array}$ & $\begin{array}{l}\text { Minimum } \\
\text { Minimum }\end{array}$ & $\begin{array}{l}.90 \\
.94\end{array}$ & $\begin{array}{l}\left(\mathrm{ft}^{3} / \mathrm{s}\right) / \mathrm{mi} \\
\left(\mathrm{ft}^{3} / \mathrm{s}\right) / \mathrm{mi}\end{array}$ & $\begin{array}{rr}2 & 2.45 \\
i^{2} & .89\end{array}$ & $\begin{array}{l}\text { Inches } \\
\text { Inches }\end{array}$ & $\begin{array}{l}33.3 \\
12.0\end{array}$ \\
\hline
\end{tabular}


Table 3.--Physical characteristics and concentrations of chemical constituents of streamflow at station number 01174565, West Branch Swift River near Shutesbury, Massachusetts

[ $\mathrm{ft}^{3} / \mathrm{s}$, cubic feet per second; $\mu \mathrm{S} / \mathrm{cm}$, microsiemens per centimeter at 25 degrees Celsius; ${ }^{\circ} \mathrm{C}$, degrees Celsius; $\mathrm{mg} / \mathrm{L}$, milligrams per liter; $\mu \mathrm{g} / \mathrm{L}$, micrograms per liter; $<$, less than; dashes indicate not analyzed]

\begin{tabular}{|c|c|c|c|c|c|c|c|c|c|}
\hline Date & Time & $\begin{array}{l}\text { Stream- } \\
\text { flow, } \\
\text { instan- } \\
\text { taneous } \\
\left(\mathrm{ft}^{3} / \mathrm{s}\right)\end{array}$ & $\begin{array}{l}\text { Spe- } \\
\text { cific } \\
\text { con- } \\
\text { duct- } \\
\text { ance } \\
(\mu \mathrm{s} / \mathrm{cm})\end{array}$ & $\begin{array}{l}\mathrm{pH} \\
\text { (stand- } \\
\text { ard } \\
\text { units) }\end{array}$ & $\begin{array}{l}\text { Temper- } \\
\text { ature } \\
\left({ }^{\circ} \mathrm{C}\right)\end{array}$ & $\begin{array}{l}\text { Hard- } \\
\text { ness } \\
(\mathrm{mg} / \mathrm{L} \\
\text { as } \\
\left.\mathrm{CaCO}_{3}\right)\end{array}$ & $\begin{array}{l}\text { Calcium, } \\
\text { dis- } \\
\text { solved } \\
\text { (mg/L } \\
\text { as } \mathrm{Ca} \text { ) }\end{array}$ & $\begin{array}{l}\text { Magne- } \\
\text { sium, } \\
\text { dis- } \\
\text { solved } \\
\text { (mg/L } \\
\text { as Mg) }\end{array}$ & $\begin{array}{l}\text { Sodium, } \\
\text { dis- } \\
\text { solved } \\
\text { (mg/L } \\
\text { as } \mathrm{Na} \text { ) }\end{array}$ \\
\hline $\begin{array}{l}11-01-83 \\
11-08-83 \\
11-15-83 \\
11-22-83 \\
11-29-83\end{array}$ & $\begin{array}{l}10: 20 \\
13: 15 \\
10: 00 \\
10: 00 \\
15: 00\end{array}$ & $\begin{array}{l}2.6 \\
3.6 \\
8.1 \\
40 \\
64\end{array}$ & $\begin{array}{l}39 \\
44 \\
42 \\
36 \\
37\end{array}$ & $\begin{array}{l}6.2 \\
6.9 \\
6.3 \\
5.7 \\
6.0\end{array}$ & $\begin{array}{l}3.5 \\
4.5 \\
3.5 \\
6.0 \\
4.5\end{array}$ & $\begin{array}{l}9 \\
9 \\
9 \\
8 \\
8\end{array}$ & $\begin{array}{l}2.6 \\
2.7 \\
2.7 \\
2.4 \\
2.3\end{array}$ & $\begin{array}{r}0.63 \\
.57 \\
.55 \\
.43 \\
.45\end{array}$ & $\begin{array}{l}3.3 \\
3.5 \\
2.9 \\
2.2 \\
2.7\end{array}$ \\
\hline $\begin{array}{l}12-06-83^{1} \\
12-13-83 \\
12-20-83 \\
12-27-83^{1} \\
01-03-84\end{array}$ & $\begin{array}{l}13: 15 \\
09: 15 \\
15: 00 \\
15: 00 \\
16: 00\end{array}$ & $\begin{array}{r}22 \\
165 \\
38 \\
24 \\
27\end{array}$ & $\begin{array}{l}34 \\
31 \\
33 \\
33 \\
--\end{array}$ & $\begin{array}{l}6.2 \\
5.7 \\
6.1 \\
6.1 \\
6.3\end{array}$ & $\begin{array}{r}2.5 \\
2.0 \\
1.0 \\
.5 \\
.5\end{array}$ & $\begin{array}{l}8 \\
7 \\
8 \\
8 \\
8\end{array}$ & $\begin{array}{l}2.4 \\
2.0 \\
2.3 \\
2.3 \\
2.3\end{array}$ & $\begin{array}{l}.45 \\
.41 \\
.47 \\
.51 \\
.52\end{array}$ & $\begin{array}{l}2.7 \\
2.5 \\
2.6 \\
2.9 \\
2.8\end{array}$ \\
\hline $\begin{array}{l}01-10-84 \\
01-17-84 \\
01-24-84 \\
01-27-84 \\
01-31-84\end{array}$ & $\begin{array}{l}14: 00 \\
08: 30 \\
10: 30 \\
16: 00 \\
09: 45\end{array}$ & $\begin{array}{l}19 \\
14 \\
12 \\
23 \\
17\end{array}$ & $\begin{array}{l}-\overline{36} \\
\overline{33} \\
--\end{array}$ & $\begin{array}{l}6.4 \\
6.6 \\
6.6 \\
5.7 \\
6.5\end{array}$ & $\begin{array}{r}.5 \\
.5 \\
.5 \\
-.5\end{array}$ & $\begin{array}{l}8 \\
8 \\
8 \\
6 \\
8\end{array}$ & $\begin{array}{l}2.4 \\
2.4 \\
2.4 \\
1.8 \\
2.3\end{array}$ & $\begin{array}{l}.49 \\
.44 \\
.54 \\
.40 \\
.45\end{array}$ & $\begin{array}{l}3.8 \\
3.1 \\
3.2 \\
2.6 \\
3.7\end{array}$ \\
\hline $\begin{array}{l}02-05-84 \\
02-05-84^{2} \\
02-07-84 \\
02-14-84 \\
02-15-84\end{array}$ & $\begin{array}{l}08: 15 \\
08: 30 \\
09: 00 \\
14: 00 \\
13: 45\end{array}$ & $\begin{array}{l}44 \\
-- \\
28 \\
28 \\
76\end{array}$ & $\begin{array}{l}-- \\
-- \\
-- \\
29 \\
34\end{array}$ & $\begin{array}{l}6.1 \\
-- \\
6.4 \\
6.0 \\
5.9\end{array}$ & $\begin{array}{r}.5 \\
-.5 \\
.5 \\
.5\end{array}$ & $\begin{array}{r}7 \\
-- \\
7 \\
7 \\
7\end{array}$ & $\begin{array}{l}2.2 \\
-- \\
2.2 \\
2.1 \\
2.1\end{array}$ & $\begin{array}{r}. .45 \\
-.46 \\
.49 \\
.45\end{array}$ & $\begin{array}{l}2.9 \\
-- \\
3.1 \\
2.8 \\
3.2\end{array}$ \\
\hline $\begin{array}{l}02-16-84 \\
02-16-84^{2} \\
02-17-84 \\
02-21-84 \\
02-28-84\end{array}$ & $\begin{array}{l}16: 00 \\
16: 05 \\
09: 30 \\
14: 00 \\
12: 00\end{array}$ & $\begin{array}{r}310 \\
-- \\
143 \\
83 \\
57\end{array}$ & $\begin{array}{l}26 \\
-- \\
26 \\
39 \\
31\end{array}$ & $\begin{array}{c}5.1 \\
-- \\
5.5 \\
6.1 \\
6.1\end{array}$ & $\begin{array}{r}.5 \\
-.5 \\
.5 \\
.5\end{array}$ & $\begin{array}{r}5 \\
-- \\
6 \\
6 \\
7\end{array}$ & $\begin{array}{l}1.5 \\
-- \\
1.8 \\
1.9 \\
2.1\end{array}$ & $\begin{array}{r}.31 \\
. .41 \\
.39 \\
.38\end{array}$ & $\begin{array}{l}1.8 \\
-- \\
2.1 \\
2.2 \\
2.8\end{array}$ \\
\hline $\begin{array}{l}03-06-84 \\
03-13-84 \\
03-20-84 \\
03-27-84 \\
03-27-84^{2}\end{array}$ & $\begin{array}{l}07: 45 \\
11: 00 \\
09: 00 \\
10: 15 \\
10: 20\end{array}$ & $\begin{array}{l}35 \\
25 \\
38 \\
42 \\
--\end{array}$ & $\begin{array}{l}-- \\
34 \\
30 \\
32 \\
--\end{array}$ & $\begin{array}{l}6.0 \\
6.2 \\
6.2 \\
6.1 \\
--\end{array}$ & $\begin{array}{r}.5 \\
.5 \\
1.0 \\
2.0 \\
--\end{array}$ & $\begin{array}{r}8 \\
8 \\
7 \\
6 \\
--\end{array}$ & $\begin{array}{l}2.2 \\
2.4 \\
2.1 \\
1.9 \\
--\end{array}$ & $\begin{array}{r}.49 \\
.50 \\
.39 \\
.42\end{array}$ & $\begin{array}{l}3.3 \\
3.4 \\
3.0 \\
2.6 \\
--\end{array}$ \\
\hline $\begin{array}{l}04-03-84 \\
04-03-84^{2} \\
04-05-84 \\
04-05-84^{2} \\
04-06-84\end{array}$ & $\begin{array}{l}14: 30 \\
14: 40 \\
16: 00 \\
16: 10 \\
10: 45\end{array}$ & $\begin{array}{r}54 \\
-- \\
249 \\
-\overline{262}\end{array}$ & $\begin{array}{l}28 \\
-- \\
27 \\
-- \\
25\end{array}$ & $\begin{array}{c}6.2 \\
-- \\
5.4 \\
-- \\
5.3\end{array}$ & $\begin{array}{c}5.0 \\
-- \\
-1.5 \\
-1.5\end{array}$ & $\begin{array}{r}6 \\
-- \\
-5 \\
--\end{array}$ & $\begin{array}{c}1.9 \\
-\overline{1.5} \\
-- \\
1.4\end{array}$ & $\begin{array}{r}-. \\
-.31 \\
.31\end{array}$ & $\begin{array}{c}2.6 \\
-- \\
-1.9 \\
-1.7\end{array}$ \\
\hline $\begin{array}{l}04-06-84^{2} \\
04-10-84^{1} \\
04-16-84 \\
04-16-84^{2} \\
04-17-84\end{array}$ & $\begin{array}{l}10: 50 \\
14: 00 \\
13: 00 \\
13: 05 \\
08: 15\end{array}$ & $\begin{array}{r}-- \\
73 \\
152 \\
-- \\
114\end{array}$ & $\begin{array}{l}-- \\
29 \\
28 \\
-- \\
27\end{array}$ & $\begin{array}{l}-- \\
6.1 \\
5.6 \\
-- \\
5.6\end{array}$ & $\begin{array}{l}-- \\
1.5 \\
4.5 \\
-- \\
5.5\end{array}$ & $\begin{array}{r}-- \\
6 \\
5 \\
- \\
5\end{array}$ & $\begin{array}{c}-- \\
1.7 \\
1.6 \\
-- \\
1.6\end{array}$ & $\begin{array}{r}.51 \\
.25 \\
-. \\
.31\end{array}$ & $\begin{array}{l}-- \\
4.2 \\
2.1 \\
-- \\
2.0\end{array}$ \\
\hline $\begin{array}{l}04-17-84^{2} \\
04-24-84 \\
05-01-84^{1} \\
05-08-84 \\
05-16-84\end{array}$ & $\begin{array}{l}08: 20 \\
14: 45 \\
15: 00 \\
14: 00 \\
09: 15\end{array}$ & $\begin{array}{l}-- \\
54 \\
26 \\
25 \\
30\end{array}$ & $\begin{array}{l}-- \\
32 \\
37 \\
35 \\
31\end{array}$ & $\begin{array}{l}-- \\
6.1 \\
6.3 \\
6.2 \\
6.1\end{array}$ & $\begin{array}{r}-- \\
7.0 \\
11.5 \\
10.5 \\
7.5\end{array}$ & $\begin{array}{r}-- \\
7 \\
7 \\
7 \\
6\end{array}$ & $\begin{array}{r}-- \\
2.0 \\
2.2 \\
2.1 \\
1.9\end{array}$ & $\begin{array}{r}. \\
.41 \\
.44 \\
.40 \\
.39\end{array}$ & $\begin{array}{l}-\overrightarrow{2.5} \\
3.1 \\
2.9 \\
2.6\end{array}$ \\
\hline
\end{tabular}


Table 3.--Physical characteristics and concentrations of chemical constituents of streamflow at station number 01174565, West Branch Swift River near Shutesbury, Massachusetts-Continued

\begin{tabular}{|c|c|c|c|c|c|c|c|c|c|c|}
\hline Date & $\begin{array}{l}\text { Potas- } \\
\text { sium, } \\
\text { dis- } \\
\text { solved } \\
\text { (mg/L } \\
\text { as K) }\end{array}$ & $\begin{array}{c}\text { Nitro- } \\
\text { gen: } \\
\text { ammonia } \\
\text { total } \\
\text { (mg/L } \\
\text { as N) }\end{array}$ & $\begin{array}{c}\text { Nitro- } \\
\text { gen, } \\
\text { ammonia, } \\
\text { dis- } \\
\text { solved } \\
\text { (mg/L } \\
\text { as N) }\end{array}$ & $\begin{array}{c}\text { Alka- } \\
\text { linity } \\
\text { field } \\
(\mathrm{mg} / \mathrm{L} \\
\mathrm{as} \\
\left.\mathrm{CaCO}_{3}\right)\end{array}$ & $\begin{array}{l}\text { Sulfate, } \\
\text { dis- } \\
\text { solved } \\
\text { (mg/L } \\
\text { as } \mathrm{SO}_{4} \text { ) }\end{array}$ & $\begin{array}{c}\text { Nitro- } \\
\text { gen, } \\
\text { nitrate, } \\
\text { dis- } \\
\text { solved } \\
\text { (mg/L } \\
\text { as } N \text { ) }\end{array}$ & $\begin{array}{l}\text { Phos- } \\
\text { phorus, } \\
\text { ortho, } \\
\text { dis- } \\
\text { solved } \\
\text { (mg/L } \\
\text { as P) }\end{array}$ & $\begin{array}{l}\text { Chlo- } \\
\text { ride, } \\
\text { dis- } \\
\text { solved } \\
\text { (mg/L } \\
\text { as Cl) }\end{array}$ & $\begin{array}{l}\text { Fluo- } \\
\text { ride, } \\
\text { dis- } \\
\text { solved } \\
\text { (mg/L } \\
\text { as F) }\end{array}$ & $\begin{array}{l}\text { silica, } \\
\text { dis- } \\
\text { solved } \\
\text { (mg/L } \\
\text { as } \\
\mathrm{SiO}_{2} \text { ) }\end{array}$ \\
\hline $\begin{array}{l}11-01-83 \\
11-08-83 \\
11-15-83 \\
11-22-83 \\
11-29-83\end{array}$ & $\begin{array}{l}0.56 \\
.54 \\
.50 \\
.41 \\
.37\end{array}$ & $\begin{array}{l}-- \\
-- \\
-- \\
-- \\
--\end{array}$ & $\begin{array}{r}0.02 \\
.12 \\
.01 \\
.03 \\
<.01\end{array}$ & $\begin{array}{l}-- \\
-- \\
-- \\
--\end{array}$ & $\begin{array}{l}7.8 \\
8.0 \\
8.4 \\
7.9 \\
7.8\end{array}$ & $\begin{array}{l}<0.05 \\
<.05 \\
<.05 \\
<.05 \\
<.05\end{array}$ & $\begin{array}{l}<0.01 \\
<.06 \\
<.06 \\
<.06 \\
<.01\end{array}$ & $\begin{array}{l}3.6 \\
4.1 \\
3.8 \\
2.3 \\
3.2\end{array}$ & $\begin{array}{r}<0.01 \\
.04 \\
<.01 \\
<.01 \\
<.01\end{array}$ & $\begin{array}{l}8.0 \\
8.1 \\
8.0 \\
7.4 \\
7.1\end{array}$ \\
\hline $\begin{array}{l}12-06-83 \\
12-13-83 \\
12-20-83 \\
12-27-83 \\
01-03-84\end{array}$ & $\begin{array}{l}.36 \\
.33 \\
.35 \\
.37 \\
.34\end{array}$ & $\begin{array}{l}-- \\
-- \\
-- \\
-- \\
--\end{array}$ & $\begin{array}{l}<.01 \\
<.01 \\
<.01 \\
.05 \\
<.01\end{array}$ & $\begin{array}{l}-- \\
-- \\
-- \\
-- \\
--\end{array}$ & $\begin{array}{l}7.9 \\
6.8 \\
7.2 \\
7.0 \\
7.7\end{array}$ & $\begin{array}{l}-- \\
<.05 \\
-- \\
-- \\
--\end{array}$ & $\begin{array}{l}<.01 \\
<.01 \\
<.01 \\
<.01 \\
<.01\end{array}$ & $\begin{array}{l}3.4 \\
3.7 \\
2.9 \\
3.4 \\
3.7\end{array}$ & $\begin{array}{r}.01 \\
.07 \\
.08 \\
.08 \\
<.01\end{array}$ & $\begin{array}{l}8.0 \\
6.5 \\
7.8 \\
8.2 \\
8.0\end{array}$ \\
\hline $\begin{array}{l}01-10-84 \\
01-17-84 \\
01-24-84 \\
01-27-84 \\
01-31-84\end{array}$ & $\begin{array}{l}.37 \\
.38 \\
.41 \\
.31 \\
.38\end{array}$ & $\begin{array}{rr}-- & \\
-- & \\
-- & \\
0.014 & .014\end{array}$ & $\begin{array}{l}<.01 \\
<.01 \\
<.01 \\
<.01 \\
<\end{array}$ & $\begin{array}{l}-- \\
-- \\
-- \\
-- \\
--\end{array}$ & $\begin{array}{l}7.6 \\
7.7 \\
7.6 \\
7.4 \\
7.8\end{array}$ & $\begin{array}{l}-- \\
-- \\
-- \\
--\end{array}$ & $\begin{array}{l}<.01 \\
<.01 \\
<.01 \\
<.06 \\
--\end{array}$ & $\begin{array}{l}5.9 \\
4.4 \\
4.9 \\
4.0 \\
6.6\end{array}$ & $\begin{array}{l}<.01 \\
<.01 \\
<.01 \\
.08 \\
.06\end{array}$ & $\begin{array}{l}8.1 \\
8.6 \\
8.7 \\
7.3 \\
8.1\end{array}$ \\
\hline $\begin{array}{l}02-05-84 \\
02-05-84 \\
02-07-84 \\
02-14-84 \\
02-15-84\end{array}$ & $\begin{array}{r}.36 \\
-.33 \\
.35 \\
.37\end{array}$ & $\begin{array}{l}<.001 \\
<.001 \\
<.033 \\
<.001\end{array}$ & $\begin{array}{l}-- \\
-- \\
-- \\
--\end{array}$ & $\begin{array}{l}-- \\
-- \\
-- \\
--\end{array}$ & $\begin{array}{c}7.7 \\
-- \\
7.8 \\
7.7 \\
7.2\end{array}$ & $\begin{array}{l}-- \\
-- \\
-- \\
--\end{array}$ & $\begin{array}{l}-- \\
-- \\
-- \\
-- \\
--\end{array}$ & $\begin{array}{c}5.1 \\
-- \\
5.2 \\
4.5 \\
5.2\end{array}$ & $\begin{array}{r}.07 \\
-.07 \\
.07 \\
.05\end{array}$ & $\begin{array}{r}7.1 \\
-- \\
7.2 \\
7.2 \\
6.4\end{array}$ \\
\hline $\begin{array}{l}02-16-84 \\
02-16-84 \\
02-17-84 \\
02-21-84 \\
02-28-84\end{array}$ & $\begin{array}{r}.35 \\
.36 \\
.34 \\
.34\end{array}$ & $\begin{array}{r}<.001 \\
<- \\
<.001 \\
.047 \\
<.001\end{array}$ & $\begin{array}{l}-- \\
-- \\
-- \\
--\end{array}$ & $\begin{array}{l}-- \\
-- \\
-- \\
--\end{array}$ & $\begin{array}{c}6.3 \\
-- \\
6.7 \\
7.2 \\
7.0\end{array}$ & $\begin{array}{l}-- \\
-- \\
-- \\
-- \\
--\end{array}$ & $\begin{array}{l}-- \\
-- \\
-- \\
--\end{array}$ & $\begin{array}{c}2.8 \\
- \\
3.3 \\
3.4 \\
4.0\end{array}$ & $\begin{array}{r}.05 \\
-.06 \\
.05 \\
.06\end{array}$ & $\begin{array}{r}4.5 \\
-- \\
5.4 \\
6.3 \\
6.4\end{array}$ \\
\hline $\begin{array}{l}03-06-84 \\
03-13-84 \\
03-20-84 \\
03-27-84 \\
03-27-84\end{array}$ & $\begin{array}{r}.38 \\
.41 \\
.37 \\
. .33 \\
--\end{array}$ & $\begin{array}{r}<.001 \\
.003 \\
<.001 \\
\\
+.021\end{array}$ & $\begin{array}{l}-- \\
-- \\
-- \\
--\end{array}$ & $\begin{array}{r}1.4 \\
1.5 \\
.8 \\
1.4 \\
--\end{array}$ & $\begin{array}{r}7.5 \\
7.5 \\
7.0 \\
7.2 \\
--\end{array}$ & $\begin{array}{l}-- \\
-- \\
-- \\
-- \\
--\end{array}$ & $\begin{array}{l}-- \\
-- \\
-- \\
-- \\
--\end{array}$ & $\begin{array}{r}6.0 \\
4.9 \\
5.1 \\
3.2 \\
--\end{array}$ & $\begin{array}{r}.04 \\
.07 \\
.06 \\
.07\end{array}$ & $\begin{array}{l}7.2 \\
8.0 \\
6.8 \\
6.2 \\
--\end{array}$ \\
\hline $\begin{array}{l}04-03-84 \\
04-03-84 \\
04-05-84 \\
04-05-84 \\
04-06-84\end{array}$ & $\begin{array}{l}-.34 \\
-- \\
- \\
- \\
.34\end{array}$ & $\begin{array}{r}.027 \\
-.191 \\
- \\
<.001\end{array}$ & $\begin{array}{l}-- \\
-- \\
-- \\
--\end{array}$ & $--_{-}^{.3} .3$ & $\begin{array}{c}7.0 \\
-- \\
5.8 \\
-- \\
5.9\end{array}$ & $\begin{array}{l}-- \\
-- \\
-- \\
-- \\
--\end{array}$ & $\begin{array}{l}-- \\
-- \\
-- \\
-- \\
--\end{array}$ & $\begin{array}{c}3.1 \\
- \\
2.1 \\
-1.8\end{array}$ & $\begin{array}{l}-.08 \\
<.01 \\
-- \\
<.01\end{array}$ & $\begin{array}{r}6.1 \\
-- \\
4.7 \\
-- \\
4.4\end{array}$ \\
\hline $\begin{array}{l}04-06-84 \\
04-10-84 \\
04-16-84 \\
04-16-84 \\
04-17-84\end{array}$ & $\begin{array}{r}-.49 \\
.28 \\
-. \\
.28\end{array}$ & $\begin{array}{r}-- \\
<.001 \\
.013 \\
-\quad .016\end{array}$ & $\begin{array}{l}-- \\
-- \\
-- \\
--\end{array}$ & $\begin{array}{r}-1.0 \\
.9 \\
-.6\end{array}$ & $\begin{array}{c}-- \\
7.2 \\
6.7 \\
-- \\
6.7\end{array}$ & $\begin{array}{l}-- \\
-- \\
-- \\
-- \\
--\end{array}$ & $\begin{array}{l}-- \\
-- \\
-- \\
-- \\
--\end{array}$ & $\begin{array}{c}-- \\
3.0 \\
2.2 \\
-- \\
2.2\end{array}$ & $\begin{array}{r}.07 \\
.05 \\
- \\
.05\end{array}$ & $\begin{array}{r}-- \\
3.9 \\
5.4 \\
-- \\
5.3\end{array}$ \\
\hline $\begin{array}{l}04-17-84 \\
04-24-84 \\
05-01-84 \\
05-08-84 \\
05-16-84\end{array}$ & $\begin{array}{r}.32 \\
.38 \\
.37 \\
.31\end{array}$ & $\begin{array}{r}- \\
.039 \\
.039 \\
.274 \\
.240\end{array}$ & $\begin{array}{l}-- \\
-- \\
-- \\
--\end{array}$ & $\begin{array}{r}-1.0 \\
1.8 \\
1.9 \\
.9\end{array}$ & $\begin{array}{l}-- \\
6.9 \\
7.0 \\
6.7 \\
6.3\end{array}$ & $\begin{array}{l}-- \\
-- \\
-- \\
-- \\
--\end{array}$ & $\begin{array}{l}-- \\
-- \\
-- \\
-- \\
--\end{array}$ & $\begin{array}{l}-- \\
3.1 \\
4.1 \\
3.8 \\
2.8\end{array}$ & $\begin{array}{r}.06 \\
.06 \\
.05 \\
.05\end{array}$ & $\begin{array}{l}-- \\
6.0 \\
6.3 \\
5.9 \\
5.9\end{array}$ \\
\hline
\end{tabular}

See footnotes at end of table. 
Table 3.--Physical characteristics and concentrations of chemical constituents of streamflow at station number 01174565, West Branch Swift River near Shutesbury, Massachusetts--Continued

\begin{tabular}{|c|c|c|c|c|c|c|c|c|c|c|}
\hline Date & $\begin{array}{c}\text { Solids, } \\
\text { sum of } \\
\text { consti- } \\
\text { tuents, } \\
\text { dis- } \\
\text { solved } \\
\text { (mg/L) }\end{array}$ & $\begin{array}{l}\text { Alum- } \\
\text { inum, } \\
\text { total } \\
\text { recov- } \\
\text { erable } \\
(\mu g / L \\
\text { as } A l)\end{array}$ & $\begin{array}{l}\text { Alum- } \\
\text { inum, } \\
\text { dis- } \\
\text { solved } \\
\text { ( } \mu \mathrm{g} / \mathrm{L} \\
\text { as Al) }\end{array}$ & $\begin{array}{c}\text { Arsenic, } \\
\text { dis- } \\
\text { solved } \\
\text { ( } \mu \text { g/L } \\
\text { as As) }\end{array}$ & $\begin{array}{l}\text { Arsenic, } \\
\text { total } \\
(\mu \mathrm{g} / \mathrm{L} \\
\text { as As) }\end{array}$ & $\begin{array}{c}\text { Copper, } \\
\text { total } \\
\text { recov- } \\
\text { erable } \\
(\mu \mathrm{g} / \mathrm{L} \\
\text { as Cu) }\end{array}$ & $\begin{array}{l}\text { Copper, } \\
\text { dis- } \\
\text { solved } \\
(\mu g / L \\
\text { as Cu) }\end{array}$ & $\begin{array}{l}\text { Iron, } \\
\text { total } \\
\text { recov- } \\
\text { erable } \\
(\mu \mathrm{g} / \mathrm{L} \\
\text { as } \mathrm{Fe})\end{array}$ & $\begin{array}{l}\text { Iron, } \\
\text { dis- } \\
\text { solved } \\
(\mu \mathrm{g} / \mathrm{L} \\
\text { as } \mathrm{Fe})\end{array}$ & $\begin{array}{l}\text { "Lead, } \\
\text { total } \\
\text { recov- } \\
\text { erable } \\
(\mu \mathrm{g} / \mathrm{L} \\
\text { as } \mathrm{Pb})\end{array}$ \\
\hline
\end{tabular}

\begin{tabular}{|c|c|c|c|}
\hline $\begin{array}{l}11-01-83 \\
11-08-83 \\
11-15-83 \\
11-22-83 \\
11-29-83\end{array}$ & $\begin{array}{l}-- \\
-- \\
-- \\
-- \\
--\end{array}$ & $\begin{array}{c}-- \\
-- \\
-- \\
-- \\
230^{\circ}\end{array}$ & $\begin{array}{r}<10 \\
70 \\
110 \\
220 \\
210\end{array}$ \\
\hline $\begin{array}{l}12-06-83 \\
12-13-83 \\
12-20-83 \\
12-27-83 \\
01-03-84\end{array}$ & $\begin{array}{l}-- \\
-- \\
-- \\
-- \\
--\end{array}$ & $\begin{array}{r}-- \\
290 \\
-- \\
-- \\
--\end{array}$ & $\begin{array}{r}140 \\
170 \\
130 \\
120 \\
20\end{array}$ \\
\hline $\begin{array}{l}01-10-84 \\
01-17-84 \\
01-24-84 \\
01-27-84 \\
01-31-84\end{array}$ & $\begin{array}{l}-- \\
-- \\
-- \\
24 \\
--\end{array}$ & $\begin{array}{l}-- \\
-- \\
-- \\
-- \\
--\end{array}$ & $\begin{array}{r}90 \\
150 \\
120 \\
140 \\
130\end{array}$ \\
\hline $\begin{array}{l}02-05-84 \\
02-05-84 \\
02-07-84 \\
02-14-84 \\
02-15-84\end{array}$ & $\begin{array}{l}-- \\
-- \\
-- \\
-- \\
--\end{array}$ & $\begin{array}{r}250 \\
-- \\
-- \\
-- \\
--\end{array}$ & $\begin{array}{r}240 \\
2110 \\
150 \\
90 \\
110\end{array}$ \\
\hline $\begin{array}{l}02-16-84 \\
02-16-84 \\
02-17-84 \\
02-21-84 \\
02-28-84\end{array}$ & $\begin{array}{l}-- \\
-- \\
21 \\
-- \\
24\end{array}$ & $\begin{array}{r}-- \\
-- \\
250 \\
-- \\
--\end{array}$ & $\begin{array}{r}200 \\
2190 \\
170 \\
250 \\
140\end{array}$ \\
\hline $\begin{array}{l}03-06-84 \\
03-13-84 \\
03-20-84 \\
03-27-84 \\
03-27-84\end{array}$ & $\begin{array}{l}28 \\
28 \\
26 \\
23 \\
--\end{array}$ & $\begin{array}{l}-- \\
-- \\
-- \\
-- \\
--\end{array}$ & $\begin{array}{r}120 \\
90 \\
110 \\
180 \\
230\end{array}$ \\
\hline $\begin{array}{l}04-03-84 \\
04-03-84 \\
04-05-84 \\
04-05-84 \\
04-06-84\end{array}$ & $\begin{array}{l}22 \\
-- \\
-- \\
-- \\
16\end{array}$ & $\begin{array}{r}-- \\
-- \\
540 \\
-- \\
250\end{array}$ & $\begin{array}{r}2<70 \\
<70 \\
2-- \\
110 \\
200\end{array}$ \\
\hline $\begin{array}{l}04-06-84 \\
04-10-84 \\
04-16-84 \\
04-16-84 \\
04-17-84\end{array}$ & $\begin{array}{l}-- \\
22 \\
19 \\
-- \\
19\end{array}$ & $\begin{array}{r}-- \\
-- \\
180 \\
-- \\
--\end{array}$ & $\begin{array}{r}290 \\
50 \\
130 \\
2150 \\
150\end{array}$ \\
\hline $\begin{array}{l}04-17-84 \\
04-24-84 \\
05-01-84 \\
05-08-84 \\
05-16-84\end{array}$ & $\begin{array}{l}-- \\
22 \\
25 \\
24 \\
21\end{array}$ & $\begin{array}{l}-- \\
-- \\
-- \\
-- \\
--\end{array}$ & $\begin{array}{r}2140 \\
30 \\
-- \\
80 \\
--\end{array}$ \\
\hline
\end{tabular}

\begin{tabular}{rr}
1 & \\
$<1$ & - \\
$<1$ & - \\
$<1$ & - \\
1 & 1 \\
$<1$ & - \\
$<1$ & $<1$ \\
$<1$ & - \\
$<1$ & - \\
$<1$ & - \\
$<1$ & - \\
$<1$ & - \\
$<1$ & - \\
$<1$ & - \\
1 & - \\
$<1$ & $<1$ \\
-- & - \\
$<1$ & - \\
$<1$ & - \\
$<1$ & - \\
$<1$ & - \\
-- & - \\
$<1$ & $<1$ \\
1 & - \\
1 & - \\
$<1$ & - \\
1 & - \\
1 & - \\
1 & - \\
-- & - \\
1 & - \\
-- & - \\
-- & - \\
-- & - \\
1 & 1 \\
-- & - \\
\hline 1 & - \\
1 & - \\
-- & - \\
1 & - \\
-- & - \\
1 & - \\
-- & - \\
$<1$ & - \\
-- & - \\
& \\
\hline & - \\
\hline & - \\
\hline & -
\end{tabular}

\begin{tabular}{rr}
-- & -- \\
-- & -- \\
-- & -- \\
\hline 1 & -- \\
-- & - \\
$<1$ & - \\
-- & -- \\
-- & -- \\
-- & -- \\
-- & -- \\
-- & -- \\
-- & -- \\
-- & -- \\
-- & -- \\
$<1$ & 6 \\
-- & -- \\
-- & -- \\
-- & -- \\
-- & -- \\
-- & -- \\
-- & -- \\
$<1$ & 2 \\
-- & -- \\
-- & -- \\
-- & -- \\
-- & -- \\
-- & -- \\
-- & -- \\
-- & -- \\
-- & -- \\
-- & -- \\
-- & 2 \\
-- & -- \\
1 & 2 \\
-- & -- \\
-- & -- \\
-1 & - \\
-- & -- \\
-- & -- \\
-- & -- \\
-- & -- \\
-- & -- \\
-- & -- \\
&
\end{tabular}

\begin{tabular}{|c|c|}
\hline 0.1 & -- \\
\hline .1 & - \\
\hline 4.8 & - \\
\hline .5 & 360 \\
\hline .2 & - \\
\hline .6 & 250 \\
\hline .3 & -- \\
\hline$\cdot 1$ & - \\
\hline 3.5 & - \\
\hline 2.5 & - \\
\hline 9.8 & - \\
\hline 5.1 & $\cdots$ \\
\hline & \\
\hline & 110 \\
\hline 23 & - \\
\hline .1 & - \\
\hline 2.5 & - \\
\hline 2.0 & - \\
\hline 7 & 180 \\
\hline 1.7 & - \\
\hline 1.8 & - \\
\hline .1 & -- \\
\hline 2.3 & - \\
\hline 3.7 & - \\
\hline .8 & - \\
\hline- & \\
\hline 2.5 & - \\
\hline 7 & 470 \\
\hline-- & - \\
\hline 2.2 & 130 \\
\hline-- & - \\
\hline 2.0 & -- \\
\hline 1.4 & 130 \\
\hline 2.0 & - \\
\hline-- & - \\
\hline .9 & - \\
\hline 3.0 & - \\
\hline & \\
\hline
\end{tabular}

63
47
48

$\begin{array}{r}-- \\ -- \\ -- \\ 6 \\ -- \\ 5 \\ -- \\ -- \\ -- \\ -- \\ -- \\ -- \\ -- \\ 9 \\ -- \\ -- \\ -- \\ -- \\ \hline 2 \\ -- \\ -- \\ -- \\ -- \\ -- \\ -- \\ -- \\ -- \\ -2 \\ - \\ \hline \\ -- \\ \hline \\ -- \\ -- \\ -- \\ -- \\ -- \\ \hline\end{array}$

See footnotes at end of table. 


\begin{tabular}{|c|c|c|c|c|c|c|c|c|c|c|}
\hline Date & $\begin{array}{l}\text { Lead, } \\
\text { dis- } \\
\text { solved } \\
(\mu g / L \\
\text { as } \mathrm{Pb})\end{array}$ & $\begin{array}{l}\text { Manga- } \\
\text { nese, } \\
\text { total } \\
\text { recov- } \\
\text { erable } \\
(\mu g / L \\
\text { as } M n)\end{array}$ & $\begin{array}{l}\text { Manga- } \\
\text { nese, } \\
\text { dis- } \\
\text { solved } \\
(\mu g / L \\
\text { as } M n)\end{array}$ & $\begin{array}{l}\text { Mercury, } \\
\text { total } \\
\text { recov- } \\
\text { erable } \\
(\mu g / L \\
\text { as } \mathrm{Hg})\end{array}$ & $\begin{array}{l}\text { Mercury, } \\
\text { dis- } \\
\text { solved } \\
\text { ( } \mu g / L \\
\text { as } \mathrm{Hg})\end{array}$ & $\begin{array}{l}\text { Sele- } \\
\text { nium, } \\
\text { total } \\
(\mu \mathrm{g} / \mathrm{L} \\
\mathrm{as} \mathrm{Se})\end{array}$ & $\begin{array}{l}\text { Sele- } \\
\text { nium, } \\
\text { dis- } \\
\text { solved } \\
(\mu g / L \\
\text { as Se) }\end{array}$ & $\begin{array}{l}\text { Vana- } \\
\text { dium, } \\
\text { dis- } \\
\text { solved } \\
(\mu \mathrm{g} / \mathrm{L} \\
\text { as V) }\end{array}$ & $\begin{array}{c}\text { Carbon, } \\
\text { organic } \\
\text { total } \\
\text { (mg/L } \\
\text { as C) }\end{array}$ & $\begin{array}{l}\text { Carbon, } \\
\text { organic, } \\
\text { dis- } \\
\text { solved } \\
\text { (mg/L } \\
\text { as C) }\end{array}$ \\
\hline $11-01-83$ & 0.8 & -- & 25 & -- & 0.2 & -- & $<1$ & $<1$ & -- & -- \\
\hline $11-08-83$ & 5.8 & -- & 15 & -- & .3 & -- & $<1$ & $<1$ & -- & -- \\
\hline $11-15-83$ & .3 & -- & 25 & -- & .1 & -- & $<1$ & $<1$ & -- & -- \\
\hline $11-22-83$ & .9 & -- & 68 & -- & .3 & -- & $<1$ & 2 & 5.7 & -- \\
\hline $11-29-83$ & .1 & 60 & 55 & $<0.1$ & $<.1$ & $<1$ & $<1$ & 1 & 4.0 & -- \\
\hline $12-06-83$ & .5 & -- & 35 & -- & $<.1$ & -- & $<1$ & $<1$ & -- & -- \\
\hline $12-13-83$ & .9 & 50 & 45 & $<.1$ & $<.1$ & $<1$ & $<1$ & $<1$ & 4.5 & -- \\
\hline $12-20-83$ & .7 & -- & 40 & -- & $<.1$ & -- & $<1$ & $<1$ & -- & -- \\
\hline $12-27-83$ & 3.9 & -- & 43 & -- & .2 & -- & $<1$ & $<1$ & -- & 2.2 \\
\hline $01-03-84$ & .4 & -- & 35 & -- & .2 & -- & $<1$ & $<1$ & -- & 1.5 \\
\hline $01-10-84$ & 1.5 & -- & 28 & -- & $<.1$ & -- & $<1$ & $<1$ & -- & 3.5 \\
\hline $01-17-84$ & .3 & -- & 26 & -- & $<.1$ & -- & $<\overline{1}$ & $<\overline{1}$ & -- & 2.4 \\
\hline $01-24-84$ & 9.0 & -- & 22 & -- & .2 & -- & $<1$ & $<1$ & 2.0 & -- \\
\hline $01-27-84$ & 5.8 & -- & 38 & -- & $<.1$ & -- & 2 & $<1$ & - & -- \\
\hline $01-31-84$ & 1.6 & -- & 30 & -- & $<.1$ & -- & $<1$ & $<1$ & -- & 9.4 \\
\hline $02-05-84$ & 1.4 & 50 & $2^{49}$ & $<.1$ & $<.1$ & $<1$ & $<1$ & $<1$ & -- & 2.7 \\
\hline $02-05-84$ & -- & -- & ${ }^{2} 21$ & -- & -- & -- & -- & -- & -- & -- \\
\hline $02-07-84$ & 4.2 & -- & 45 & -- & $<.1$ & -- & $<1$ & $<1$ & -- & 2.6 \\
\hline $02-14-84$ & .3 & -- & 41 & -- & $<.1$ & -- & $<1$ & $<1$ & -- & 2.3 \\
\hline $02-15-84$ & .8 & -- & 53 & -- & $<.1$ & -- & $<1$ & $<1$ & -- & 2.8 \\
\hline $02-16-84$ & 1.5 & -- & 120 & -- & .1 & -- & $<1$ & 4 & -- & 5.2 \\
\hline $02-16-84$ & - & -- & 296 & -- & -- & -- & - & -- & -- & -- \\
\hline $02-17-84$ & 1.6 & 100 & 99 & $<.1$ & $<.1$ & $<1$ & $<1$ & 1 & -- & 4.1 \\
\hline $02-21-84$ & .8 & -- & 65 & -- & $<.1$ & -- & $<1$ & $<1$ & -- & 3.3 \\
\hline $02-28-84$ & .7 & -- & 39 & -- & $<.1$ & -- & $<1$ & $<1$ & 2.2 & -- \\
\hline $03-06-84$ & .8 & -- & 32 & -- & $<.1$ & -- & $<1$ & $<1$ & 2.4 & -- \\
\hline $03-13-84$ & .8 & -- & 26 & -- & .1 & -- & $<1$ & 1 & 1.7 & -- \\
\hline $03-20-84$ & 1.1 & -- & 34 & -- & .1 & -- & $<1$ & $<1$ & 1.8 & -- \\
\hline $03-27-84$ & .7 & -- & 38 & -- & $\therefore 1$ & -- & $<1$ & $<1$ & 2.0 & -- \\
\hline $03-27-84$ & -- & -- & 227 & -- & -- & -- & -- & -- & -- & -- \\
\hline $04-03-84$ & .6 & -- & 34 & -- & $<.1$ & -- & $<1$ & $<1$ & 1.8 & -- \\
\hline $04-03-84$ & -- & -- & 234 & -- & -- & -- & - & -- & -- & -- \\
\hline $04-05-84$ & .4 & 90 & 21 & -- & -- & -- & -- & 1 & 5.4 & -- \\
\hline $04-05-84$ & -- & -- & 250 & -- & -- & -- & -- & -- & -- & -- \\
\hline $04-06-84$ & 1.0 & 80 & 76 & $<.1$ & $<.1$ & $<1$ & $<1$ & $<1$ & 7.2 & -- \\
\hline $04-06-84$ & -- & -- & 247 & -- & -- & -- & -- & -- & -- & -- \\
\hline $04-10-84$ & 1.4 & -- & 27 & -- & $<.1$ & -- & $<1$ & 3 & -- & 2.1 \\
\hline $04-16-84$ & .7 & 40 & 34 & $<.1$ & $<.1$ & $<1$ & $<1$ & $<1$ & -- & 2.5 \\
\hline $04-16-84$ & -- & - & 233 & -- & -- & -- & - & - & -- & -- \\
\hline $04-17-84$ & .4 & -- & 37 & -- & $<.1$ & -- & $<1$ & $<1$ & -- & 2.6 \\
\hline $04-17-84$ & -- & -- & $2_{37}$ & -- & -- & -- & -- & -- & -- & -- \\
\hline $04-24-84$ & .6 & -- & 31 & -- & $<.1$ & -- & $<1$ & $<1$ & -- & 1.9 \\
\hline $05-01-84$ & -- & -- & 26 & -- & -- & -- & -- & -- & -- & 1.8 \\
\hline $05-08-84$ & 1.4 & -- & 28 & -- & $<.1$ & -- & $<1$ & $<1$ & -- & 1.9 \\
\hline $05-16-84$ & -- & -- & 33 & -- & -- & -- & - & - & -- & 2.2 \\
\hline
\end{tabular}

See footnotes at end of table. 
Table 3.--Physical characteristics and concentrations of chemical constituents of streamflow at station number 01174565, West Branch Swift River near Shutesbury, Massachusetts--Continued

\begin{tabular}{|c|c|c|c|c|c|c|c|c|c|}
\hline Date & Time & $\begin{array}{l}\text { Stream- } \\
\text { flow, } \\
\text { instan- } \\
\text { taneous } \\
\left(\mathrm{ft}^{3} / \mathrm{s}\right)\end{array}$ & $\begin{array}{l}\text { Spe- } \\
\text { cific } \\
\text { con- } \\
\text { duct- } \\
\text { ance } \\
(\mu s / \mathrm{cm})\end{array}$ & $\begin{array}{c}\mathrm{pH} \\
\text { (stand- } \\
\text { ard } \\
\text { units) }\end{array}$ & $\begin{array}{l}\text { Temper- } \\
\text { ature } \\
\left({ }^{\circ} \mathrm{C}\right)\end{array}$ & $\begin{array}{l}\text { Hard- } \\
\text { hess } \\
(\mathrm{mg} / \mathrm{L} \\
\mathrm{as} \\
\left.\mathrm{CaCO}_{3}\right)\end{array}$ & $\begin{array}{l}\text { Calcium, } \\
\text { dis- } \\
\text { solved } \\
\text { (mg/L } \\
\text { as } \mathrm{Ca} \text { ) }\end{array}$ & $\begin{array}{l}\text { Magne- } \\
\text { sium, } \\
\text { dis- } \\
\text { solved } \\
\text { (mg/L } \\
\text { as Mg) }\end{array}$ & $\begin{array}{l}\text { sodium, } \\
\text { dis- } \\
\text { solved } \\
\text { (mg/L } \\
\text { as Na) }\end{array}$ \\
\hline $\begin{array}{l}05-22-84^{1} \\
05-29-84 \\
05-30-84 \\
05-30-84^{2} \\
05-31-84\end{array}$ & $\begin{array}{l}14: 00 \\
16: 45 \\
10: 30 \\
10: 40 \\
09: 30\end{array}$ & $\begin{array}{r}22 \\
193 \\
753 \\
-- \\
630\end{array}$ & $\begin{array}{l}35 \\
22 \\
23 \\
-- \\
22\end{array}$ & $\begin{array}{c}6.4 \\
5.6 \\
5.1 \\
-- \\
5.1\end{array}$ & $\begin{array}{l}14.5 \\
11.5 \\
10.5 \\
-- \\
10.5\end{array}$ & $\begin{array}{r}7 \\
5 \\
5 \\
-- \\
4\end{array}$ & $\begin{array}{c}2.2 \\
1.6 \\
1.4 \\
- \\
1.3\end{array}$ & $\begin{array}{r}0.48 \\
.36 \\
-.33 \\
-\quad .29\end{array}$ & $\begin{array}{r}3.0 \\
1.8 \\
1.5 \\
- \\
1.5\end{array}$ \\
\hline $\begin{array}{l}06-05-84 \\
06-12-84 \\
06-19-84 \\
06-26-84 \\
07-03-84\end{array}$ & $\begin{array}{l}15: 30 \\
15: 00 \\
14: 30 \\
11: 00 \\
13: 45\end{array}$ & $\begin{array}{l}60 \\
20 \\
17 \\
10 \\
6.8\end{array}$ & $\begin{array}{l}29 \\
37 \\
40 \\
42 \\
44\end{array}$ & $\begin{array}{l}6.1 \\
6.5 \\
6.7 \\
6.8 \\
6.6\end{array}$ & $\begin{array}{l}14.5 \\
17.5 \\
17.0 \\
14.0 \\
17.0\end{array}$ & $\begin{array}{l}6 \\
8 \\
8 \\
9 \\
9\end{array}$ & $\begin{array}{l}1.9 \\
2.3 \\
2.4 \\
2.7 \\
2.6\end{array}$ & $\begin{array}{l}.35 \\
.45 \\
.50 \\
.59 \\
.55\end{array}$ & $\begin{array}{l}2.3 \\
3.2 \\
3.4 \\
3.9 \\
4.0\end{array}$ \\
\hline $\begin{array}{l}07-10-84 \\
07-17-84 \\
07-24-84 \\
07-31-84 \\
08-07-84\end{array}$ & $\begin{array}{l}12: 30 \\
09: 45 \\
13: 30 \\
13: 00 \\
10: 00\end{array}$ & $\begin{array}{r}21 \\
19 \\
6.8 \\
4.9 \\
2.8\end{array}$ & $\begin{array}{l}31 \\
30 \\
36 \\
38 \\
45\end{array}$ & $\begin{array}{l}6.0 \\
6.1 \\
6.6 \\
6.5 \\
6.6\end{array}$ & $\begin{array}{l}17.0 \\
16.5 \\
20.0 \\
17.5 \\
18.0\end{array}$ & $\begin{array}{r}7 \\
7 \\
8 \\
-- \\
--\end{array}$ & $\begin{array}{l}1.9 \\
2.1 \\
2.4 \\
-- \\
--\end{array}$ & $\begin{array}{r}.43 \\
.41 \\
.47 \\
--\end{array}$ & $\begin{array}{l}2.6 \\
2.6 \\
3.5 \\
-- \\
--\end{array}$ \\
\hline $\begin{array}{l}08-14-84 \\
08-21-84 \\
08-28-84 \\
09-04-84 \\
09-11-84\end{array}$ & $\begin{array}{l}14: 15 \\
13: 45 \\
15: 00 \\
14: 15 \\
13: 15\end{array}$ & $\begin{array}{l}2.3 \\
3.4 \\
1.6 \\
2.9 \\
1.3\end{array}$ & $\begin{array}{l}46 \\
36 \\
45 \\
41 \\
--\end{array}$ & $\begin{array}{l}6.5 \\
6.6 \\
6.8 \\
6.7 \\
6.8\end{array}$ & $\begin{array}{l}20.0 \\
-- \\
17.0 \\
13.0 \\
--\end{array}$ & $\begin{array}{l}-- \\
-- \\
-- \\
-- \\
10\end{array}$ & $\begin{array}{l}-- \\
-- \\
-- \\
-- \\
3.1\end{array}$ & $\begin{array}{l}-- \\
-- \\
-- \\
-- \\
\quad .58\end{array}$ & $\begin{array}{l}-- \\
-- \\
-- \\
-- \\
4.6\end{array}$ \\
\hline $\begin{array}{l}09-18-84 \\
09-25-84^{1} \\
10-02-84 \\
10-09-84 \\
10-16-84^{1}\end{array}$ & $\begin{array}{l}13: 45 \\
14: 00 \\
14: 45 \\
09: 30 \\
13: 00\end{array}$ & $\begin{array}{r}1.3 \\
.9 \\
15 \\
1.7 \\
1.3\end{array}$ & $\begin{array}{l}47 \\
49 \\
37 \\
43 \\
47\end{array}$ & $\begin{array}{l}6.8 \\
6.8 \\
6.4 \\
6.6 \\
6.7\end{array}$ & $\begin{array}{r}10.0 \\
15.0 \\
9.0 \\
9.0 \\
9.0\end{array}$ & $\begin{array}{r}11 \\
12 \\
8 \\
9 \\
10\end{array}$ & $\begin{array}{l}3.2 \\
3.5 \\
2.5 \\
2.8 \\
3.0\end{array}$ & $\begin{array}{l}.61 \\
.69 \\
.49 \\
.56 \\
.67\end{array}$ & $\begin{array}{l}4.6 \\
5.4 \\
3.0 \\
3.9 \\
4.2\end{array}$ \\
\hline $\begin{array}{l}10-23-84 \\
10-30-84 \\
11-06-84 \\
11-13-84 \\
11-20-84\end{array}$ & $\begin{array}{l}15: 00 \\
12: 30 \\
10: 15 \\
14: 15 \\
14: 15\end{array}$ & $\begin{array}{l}3.3 \\
3.3 \\
27 \\
23 \\
6.6\end{array}$ & $\begin{array}{l}40 \\
37 \\
32 \\
33 \\
37\end{array}$ & $\begin{array}{l}6.5 \\
6.4 \\
5.6 \\
5.8 \\
6.0\end{array}$ & $\begin{array}{r}12.5 \\
10.0 \\
7.5 \\
7.0 \\
1.0\end{array}$ & $\begin{array}{r}8 \\
-- \\
-- \\
7 \\
8\end{array}$ & $\begin{array}{l}2.5 \\
-- \\
-- \\
2.1 \\
2.3\end{array}$ & $\begin{array}{l}.51 \\
-- \\
.45 \\
.50\end{array}$ & $\begin{array}{l}3.3 \\
-- \\
-- \\
2.5 \\
2.9\end{array}$ \\
\hline $\begin{array}{l}11-27-84^{1} \\
12-05-84 \\
12-11-84^{1} \\
12-18-84 \\
12-26-84\end{array}$ & $\begin{array}{l}09: 30 \\
09: 30 \\
09: 45 \\
13: 00 \\
12: 45\end{array}$ & $\begin{array}{l}4.4 \\
6.6 \\
5.8 \\
16 \\
19\end{array}$ & $\begin{array}{l}44 \\
39 \\
39 \\
34 \\
35\end{array}$ & $\begin{array}{l}6.1 \\
6.4 \\
6.2 \\
5.9 \\
5.9\end{array}$ & $\begin{array}{r}1.0 \\
.5 \\
1.0 \\
2.5 \\
.5\end{array}$ & $\begin{array}{l}9 \\
8 \\
8 \\
8 \\
7\end{array}$ & $\begin{array}{l}2.6 \\
2.4 \\
2.3 \\
2.2 \\
2.2\end{array}$ & $\begin{array}{l}.54 \\
.55 \\
.51 \\
.57 \\
.48\end{array}$ & $\begin{array}{l}3.3 \\
3.3 \\
3.0 \\
2.9 \\
2.8\end{array}$ \\
\hline $\begin{array}{l}01-02-85 \\
01-08-85 \\
01-16-85 \\
01-22-85 \\
01-29-85\end{array}$ & $\begin{array}{l}10: 30 \\
13: 30 \\
11: 00 \\
13: 00 \\
12: 45\end{array}$ & $\begin{array}{r}31 \\
12 \\
7.4 \\
4.1 \\
1.9\end{array}$ & $\begin{array}{l}34 \\
-- \\
42 \\
38 \\
35\end{array}$ & $\begin{array}{l}5.8 \\
6.3 \\
6.1 \\
6.1 \\
6.3\end{array}$ & $\begin{array}{r}2.5 \\
.5 \\
.5 \\
.5 \\
.5\end{array}$ & $\begin{array}{l}7 \\
8 \\
9 \\
8 \\
8\end{array}$ & $\begin{array}{l}2.1 \\
2.4 \\
2.6 \\
2.5 \\
2.4\end{array}$ & $\begin{array}{l}.45 \\
.45 \\
.54 \\
.52 \\
.52\end{array}$ & $\begin{array}{l}2.6 \\
2.7 \\
2.9 \\
2.9 \\
3.0\end{array}$ \\
\hline $\begin{array}{l}02-05-85 \\
02-13-85 \\
02-19-85 \\
02-25-85 \\
03-05-85\end{array}$ & $\begin{array}{l}13: 00 \\
15: 30 \\
14: 00 \\
09: 30 \\
09: 30\end{array}$ & $\begin{array}{l}2.5 \\
36 \\
9.9 \\
40 \\
16\end{array}$ & $\begin{array}{l}39 \\
39 \\
35 \\
30 \\
36\end{array}$ & $\begin{array}{l}6.3 \\
6.1 \\
6.1 \\
5.6 \\
6.2\end{array}$ & $\begin{array}{r}.5 \\
.5 \\
1.0 \\
1.0 \\
.0\end{array}$ & $\begin{array}{l}9 \\
8 \\
8 \\
7 \\
8\end{array}$ & $\begin{array}{l}2.6 \\
2.5 \\
2.3 \\
2.0 \\
2.3\end{array}$ & $\begin{array}{l}.57 \\
.52 \\
.45 \\
.43 \\
.53\end{array}$ & $\begin{array}{l}3.1 \\
3.4 \\
3.0 \\
2.6 \\
3.0\end{array}$ \\
\hline
\end{tabular}


Table 3.--Physical characteristics and concentrations of chemical constituents of streamflow at station number 01174565, West Branch Swift River near Shutesbury, Massachusetts--Continued

\begin{tabular}{|c|c|c|c|c|c|c|c|c|c|c|}
\hline Date & $\begin{array}{l}\text { Potas- } \\
\text { sium, } \\
\text { dis- } \\
\text { solved } \\
\text { (mg/L } \\
\text { as K) }\end{array}$ & $\begin{array}{c}\text { Nitro- } \\
\text { gen, } \\
\text { ammonia } \\
\text { total } \\
\text { (mg/L } \\
\text { as N) }\end{array}$ & $\begin{array}{l}\text { Nitro- } \\
\text { gen, } \\
\text { ammonia, } \\
\text { dis- } \\
\text { solved } \\
\text { (mg/L } \\
\text { as N) }\end{array}$ & $\begin{array}{c}\text { Alka- } \\
\text { linity } \\
\text { field } \\
\text { (mg/L } \\
\text { as } \\
\mathrm{CaCO}_{3} \text { ) }\end{array}$ & $\begin{array}{l}\text { Sulfate, } \\
\text { dis- } \\
\text { solved } \\
(\mathrm{mg} / \mathrm{L} \\
\text { as } \mathrm{SO}_{4} \text { ) }\end{array}$ & $\begin{array}{l}\text { Nitro- } \\
\text { gen, } \\
\text { nitrate, } \\
\text { dis- } \\
\text { solved } \\
\text { (mg/L } \\
\text { as N) }\end{array}$ & $\begin{array}{l}\text { Phos- } \\
\text { phorus, } \\
\text { ortho, } \\
\text { dis- } \\
\text { solved } \\
\text { (mg/L } \\
\text { as P) }\end{array}$ & $\begin{array}{l}\text { Chlo- } \\
\text { ride, } \\
\text { dis- } \\
\text { solved } \\
\text { (mg/L } \\
\text { as Cl) }\end{array}$ & $\begin{array}{l}\text { Fluo- } \\
\text { ride, } \\
\text { dis- } \\
\text { solved } \\
\text { (mg/L } \\
\text { as F) }\end{array}$ & $\begin{array}{c}\text { silica, } \\
\text { dis- } \\
\text { solved } \\
\text { (mg/L } \\
\text { as } \\
\mathrm{SiO}_{2} \text { ) }\end{array}$ \\
\hline $\begin{array}{l}05-22-84 \\
05-29-84 \\
05-30-84 \\
05-30-84 \\
05-31-84\end{array}$ & $\begin{array}{r}0.37 \\
.29 \\
.33 \\
-\quad .38 \\
.28\end{array}$ & $\begin{array}{r}<0.001 \\
.024 \\
.038 \\
-\quad \\
.078\end{array}$ & $\begin{array}{l}-- \\
-- \\
-- \\
-- \\
--\end{array}$ & $\begin{array}{r}1.4 \\
.6 \\
.2 \\
--\end{array}$ & $\begin{array}{r}7.1 \\
5.9 \\
5.1 \\
-- \\
5.6\end{array}$ & $\begin{array}{l}-- \\
-- \\
-- \\
-- \\
--\end{array}$ & $\begin{array}{l}-- \\
-- \\
-- \\
-- \\
--\end{array}$ & $\begin{array}{c}3.7 \\
1.9 \\
1.5 \\
-- \\
1.3\end{array}$ & $\begin{array}{r}0.05 \\
.05 \\
-.05 \\
-\quad .04\end{array}$ & $\begin{array}{r}6.1 \\
5.2 \\
4.6 \\
- \\
4.9\end{array}$ \\
\hline $\begin{array}{l}06-05-84 \\
06-12-84 \\
06-19-84 \\
06-26-84 \\
07-03-84\end{array}$ & $\begin{array}{l}.29 \\
.38 \\
.41 \\
.44 \\
.48\end{array}$ & $\begin{array}{r}.034 \\
.274 \\
.003 \\
<.001 \\
.016\end{array}$ & $\begin{array}{l}-- \\
-- \\
-- \\
-- \\
--\end{array}$ & $\begin{array}{r}.3 \\
2.7 \\
4.1 \\
- \\
4.3\end{array}$ & $\begin{array}{l}7.2 \\
6.7 \\
6.8 \\
6.9 \\
6.8\end{array}$ & $\begin{array}{l}-- \\
-- \\
-- \\
-- \\
--\end{array}$ & $\begin{array}{l}-- \\
-- \\
-- \\
-- \\
--\end{array}$ & $\begin{array}{l}2.4 \\
3.8 \\
4.6 \\
5.2 \\
5.2\end{array}$ & $\begin{array}{l}.01 \\
.06 \\
.05 \\
.08 \\
.07\end{array}$ & $\begin{array}{l}6.9 \\
7.9 \\
8.0 \\
8.0 \\
7.6\end{array}$ \\
\hline $\begin{array}{l}07-10-84 \\
07-17-84 \\
07-24-84 \\
07-31-84 \\
08-07-84\end{array}$ & $\begin{array}{r}.28 \\
.33 \\
.36 \\
--\end{array}$ & $\begin{array}{r}.039 \\
.005 \\
.069 \\
--\end{array}$ & $\begin{array}{l}<0.01 \\
-- \\
-- \\
-- \\
--\end{array}$ & $\begin{array}{l}.5 \\
2.1 \\
2.8 \\
1.8 \\
6.4\end{array}$ & $\begin{array}{l}6.2 \\
6.2 \\
6.0 \\
-- \\
--\end{array}$ & $\begin{array}{l}<0.05 \\
-- \\
-- \\
-- \\
--\end{array}$ & $\begin{array}{l}-- \\
-- \\
-- \\
-- \\
--\end{array}$ & $\begin{array}{l}3.1 \\
3.1 \\
4.2 \\
-- \\
--\end{array}$ & $\begin{array}{r}.07 \\
.08 \\
<.01 \\
--\end{array}$ & $\begin{array}{c}7.1 \\
7.6 \\
8.0 \\
-- \\
--\end{array}$ \\
\hline $\begin{array}{l}08-14-84 \\
08-21-84 \\
08-28-84 \\
09-04-84 \\
09-11-84\end{array}$ & $\begin{array}{l}-- \\
-- \\
-- \\
-- \\
\quad .67\end{array}$ & $\begin{array}{l}-- \\
-- \\
-- \\
-- \\
<.001\end{array}$ & $\begin{array}{l}-- \\
-- \\
-- \\
-- \\
--\end{array}$ & $\begin{array}{l}5.7 \\
3.5 \\
5.7 \\
5.5 \\
6.1\end{array}$ & $\begin{array}{l}-- \\
-- \\
-- \\
-- \\
6.8\end{array}$ & $\begin{array}{l}-- \\
-- \\
-- \\
-- \\
--\end{array}$ & $\begin{array}{l}-- \\
-- \\
-- \\
-- \\
--\end{array}$ & $\begin{array}{l}-- \\
-- \\
-- \\
-- \\
6.1\end{array}$ & $\begin{array}{l}-- \\
-- \\
-- \\
-- \\
\quad .08\end{array}$ & $\begin{array}{l}-- \\
-- \\
-- \\
-- \\
7.8\end{array}$ \\
\hline $\begin{array}{l}09-18-84 \\
09-25-84 \\
10-02-84 \\
10-09-84 \\
10-16-84\end{array}$ & $\begin{aligned} .62 \\
3.76 \\
1.1 \\
.76 \\
.77\end{aligned}$ & $\begin{array}{l}.142 \\
.133 \\
.020 \\
.052 \\
.002\end{array}$ & $\begin{array}{l}-- \\
-- \\
-- \\
-- \\
--\end{array}$ & $\begin{array}{l}5.7 \\
6.3 \\
2.6 \\
3.9 \\
5.5\end{array}$ & $\begin{array}{l}7.0 \\
7.1 \\
7.5 \\
6.7 \\
6.9\end{array}$ & $\begin{array}{l}.09 \\
.09 \\
.04 \\
.03 \\
.04\end{array}$ & $\begin{array}{r}<0.01 \\
<.01 \\
<.01 \\
<.01 \\
<.01\end{array}$ & $\begin{array}{l}5.8 \\
6.6 \\
3.4 \\
4.3 \\
5.2\end{array}$ & $\begin{array}{r}.04 \\
.05 \\
.08 \\
.05 \\
<.01\end{array}$ & $\begin{array}{l}8.1 \\
8.5 \\
6.7 \\
7.6 \\
8.0\end{array}$ \\
\hline $\begin{array}{l}10-23-84 \\
10-30-84 \\
11-06-84 \\
11-13-84 \\
11-20-84\end{array}$ & $\begin{array}{r}3_{1.5} \\
-- \\
-- \\
.56 \\
.47\end{array}$ & $\begin{array}{r}-- \\
<.001 \\
<.001 \\
<.001 \\
.040\end{array}$ & $\begin{array}{l}-- \\
-- \\
-- \\
-- \\
--\end{array}$ & $\begin{array}{r}3.1 \\
2.7 \\
.4 \\
.6 \\
1.3\end{array}$ & $\begin{array}{c}7.1 \\
-- \\
-- \\
7.2 \\
7.3\end{array}$ & $\begin{array}{l}.06 \\
-- \\
-- \\
--\end{array}$ & $\begin{array}{l}<.01 \\
-- \\
-- \\
<.01 \\
<.01\end{array}$ & $\begin{array}{l}3.8 \\
-- \\
-- \\
2.9 \\
3.2\end{array}$ & $\begin{aligned} &-.08 \\
&-- \\
& .06 \\
& .05\end{aligned}$ & $\begin{array}{c}7.4 \\
-- \\
-- \\
7.3 \\
8.3\end{array}$ \\
\hline $\begin{array}{l}11-27-84 \\
12-05-84 \\
12-11-84 \\
12-18-84 \\
12-26-84\end{array}$ & $\begin{array}{l}.54 \\
.49 \\
.44 \\
.38 \\
.40\end{array}$ & $\begin{array}{r}<.001 \\
.011 \\
.034 \\
.009\end{array}$ & $\begin{array}{l}-- \\
-- \\
-- \\
-- \\
--\end{array}$ & $\begin{array}{l}2.0 \\
2.0 \\
1.9 \\
1.0 \\
1.0\end{array}$ & $\begin{array}{l}8.3 \\
8.3 \\
7.2 \\
7.3 \\
7.8\end{array}$ & $\begin{array}{l}<.05 \\
.03 \\
.03 \\
.03 \\
.02\end{array}$ & $\begin{array}{l}<.01 \\
<.01 \\
<.01 \\
<.01 \\
<.01\end{array}$ & $\begin{array}{l}4.1 \\
3.8 \\
3.3 \\
3.0 \\
3.3\end{array}$ & $\begin{array}{l}<.01 \\
.05 \\
.04 \\
.18 \\
.04\end{array}$ & $\begin{array}{l}8.7 \\
8.3 \\
8.3 \\
8.1 \\
8.0\end{array}$ \\
\hline $\begin{array}{l}01-02-85 \\
01-08-85 \\
01-16-85 \\
01-22-85 \\
01-29-85\end{array}$ & $\begin{array}{l}.35 \\
.45 \\
.42 \\
.44 \\
.44\end{array}$ & $\begin{array}{l}-- \\
-- \\
-- \\
-- \\
--\end{array}$ & $\begin{array}{l}<.01 \\
<.01 \\
.01 \\
.04 \\
.09\end{array}$ & $\begin{array}{l}.6 \\
1.4 \\
2.1 \\
2.3 \\
2.1\end{array}$ & $\begin{array}{r}6.9 \\
31 \\
8.2 \\
7.5 \\
7.5\end{array}$ & $\begin{array}{l}.01 \\
.04 \\
.01 \\
.27 \\
.02\end{array}$ & $\begin{array}{l}<.01 \\
<.01 \\
<.01 \\
<.01 \\
<.01\end{array}$ & $\begin{array}{l}3.3 \\
5.3 \\
4.1 \\
3.4 \\
3.2\end{array}$ & $\begin{array}{l}.06 \\
.41 \\
.02 \\
.10 \\
.03\end{array}$ & $\begin{array}{l}7.6 \\
8.3 \\
9.1 \\
9.0 \\
9.1\end{array}$ \\
\hline $\begin{array}{l}02-05-85 \\
02-13-85 \\
02-19-85 \\
02-25-85 \\
03-05-85\end{array}$ & $\begin{array}{l}.43 \\
.57 \\
.43 \\
.39 \\
.39\end{array}$ & $\begin{array}{l}-- \\
-- \\
-- \\
-- \\
--\end{array}$ & $\begin{array}{r}.04 \\
.02 \\
.01 \\
.03 \\
<.01\end{array}$ & $\begin{array}{r}2.5 \\
1.0 \\
1.4 \\
.6 \\
1.2\end{array}$ & $\begin{array}{r}4_{1.5} \\
8.4 \\
7.6 \\
6.6 \\
7.8\end{array}$ & $\begin{array}{l}.01 \\
.27 \\
.04 \\
.01 \\
.02\end{array}$ & $\begin{array}{l}<.01 \\
<.01 \\
<.01 \\
<.01 \\
<.01\end{array}$ & $\begin{array}{l}4.74 \\
4.3 \\
3.4 \\
2.7 \\
9.8\end{array}$ & $\begin{array}{l}<.01 \\
.41 \\
.10 \\
.05 \\
.04\end{array}$ & $\begin{array}{l}9.2 \\
7.3 \\
8.0 \\
6.9 \\
7.0\end{array}$ \\
\hline
\end{tabular}

See footnotes at end of table. 
Table 3.--Physical characteristics and concentrations of chemical constituents of streamflow at station number 01174565, West Branch Swift River near Shutesbury, Massachusetts--Continued

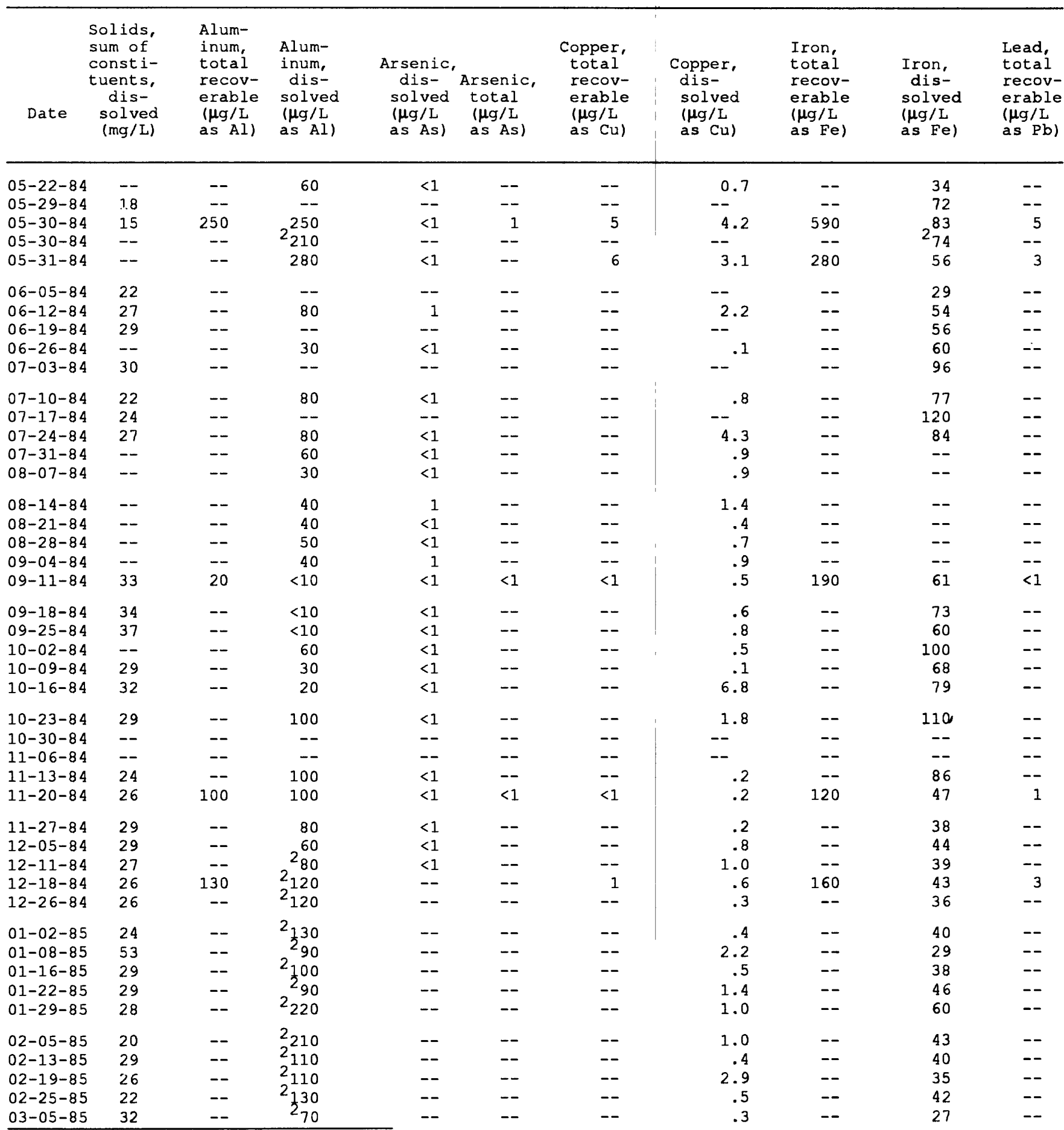

See footnotes at end of table. 


\begin{tabular}{|c|c|c|c|c|c|c|c|c|c|c|}
\hline Date & $\begin{array}{l}\text { Lead, } \\
\text { dis- } \\
\text { solved } \\
(\mu g / L \\
\text { as } \mathrm{Pb})\end{array}$ & $\begin{array}{l}\text { Manga- } \\
\text { nese, } \\
\text { total } \\
\text { recov- } \\
\text { erable } \\
(\mu g / L \\
\text { as } \mathrm{Mn})\end{array}$ & $\begin{array}{l}\text { Manga- } \\
\text { nese, } \\
\text { dis- } \\
\text { solved } \\
(\mu g / L \\
\text { as } M n)\end{array}$ & $\begin{array}{c}\text { Mercury, } \\
\text { total } \\
\text { recov- } \\
\text { erablele } \\
\text { ( } \mu \mathrm{g} / \mathrm{L} \\
\text { as } \mathrm{Hg})\end{array}$ & $\begin{array}{c}\text { Mercury, } \\
\text { dis- } \\
\text { solved } \\
(\mu g / L \\
\text { as } \mathrm{Hg})\end{array}$ & $\begin{array}{l}\text { Sele- } \\
\text { nium, } \\
\text { total } \\
(\mu \mathrm{g} / \mathrm{L} \\
\text { as Se) }\end{array}$ & $\begin{array}{l}\text { Sele- } \\
\text { nium, } \\
\text { dis- } \\
\text { solved } \\
(\mu g / L \\
\text { as } \mathrm{Se})\end{array}$ & $\begin{array}{l}\text { Vana- } \\
\text { dium, } \\
\text { dis- } \\
\text { solved } \\
(\mu g / L \\
\text { as V) }\end{array}$ & $\begin{array}{l}\text { Carbon, } \\
\text { organic } \\
\text { total } \\
\text { (mg/L } \\
\text { as C) }\end{array}$ & $\begin{array}{c}\text { Carbon, } \\
\text { organic, } \\
\text { dis- } \\
\text { solved } \\
\text { (mg/L } \\
\text { as C) }\end{array}$ \\
\hline $05-22-84$ & 0.3 & -- & 21 & -- & $<0.1$ & -- & $<1$ & $<1$ & $\cdots$ & 2.0 \\
\hline $05-29-84$ & -- & -- & 47 & -- & -- & -- & -- & -- & -- & 5.1 \\
\hline $05-30-84$ & .6 & 120 & 72 & 0.1 & .1 & $<1$ & $<1$ & $<1$ & 5.6 & 5.6 \\
\hline $05-30-84$ & -- & - & 269 & -- & -- & -- & -- & -- & -- & -- \\
\hline $05-31-84$ & .4 & -- & 48 & -- & $<.1$ & -- & $<1$ & $<1$ & 4.2 & -- \\
\hline $06-05-84$ & -- & -- & 36 & -- & -- & -- & -- & -- & -- & 2.4 \\
\hline $06-12-84$ & .7 & - & 24 & -- & $<.1$ & -- & $<1$ & $<1$ & -- & 2.1 \\
\hline $06-19-84$ & -- & -- & 17 & -- & -- & -- & - & - & -- & 2.0 \\
\hline $06-26-84$ & .8 & -- & 11 & -- & $<.1$ & -- & $<1$ & $<1$ & -- & 1.8 \\
\hline $07-03-84$ & -- & -- & 10 & -- & -- & -- & -- & -- & -- & 2.2 \\
\hline $07-10-84$ & .4 & -- & 57 & -- & $<.1$ & -- & $<1$ & $<1$ & -- & 4.1 \\
\hline $07-17-84$ & -- & -- & 43 & - & -- & -- & -- & -- & -- & 4.3 \\
\hline $07-24-84$ & .7 & -- & 11 & -- & $<.1$ & -- & $<1$ & $<1$ & -- & -- \\
\hline $07-31-84$ & 1.7 & -- & -- & -- & .3 & -- & $<1$ & $<1$ & -- & 2.6 \\
\hline $08-07-84$ & 1.2 & -- & -- & -- & $<.1$ & - & $<1$ & $<1$ & -- & -- \\
\hline $08-14-84$ & .8 & -- & -- & -- & $<.1$ & -- & $<1$ & $<1$ & -- & 2.8 \\
\hline $08-21-84$ & .6 & -- & -- & -- & $<.1$ & -- & $<1$ & $<1$ & -- & 2.7 \\
\hline $08-28-84$ & 1.5 & -- & -- & -- & $<.1$ & -- & $<1$ & $<1$ & -- & 2.0 \\
\hline $09-04-84$ & 1.4 & -- & -- & -- & $<.1$ & -- & $<1$ & $<1$ & -- & .8 \\
\hline $09-11-84$ & .6 & $<10$ & 4 & .4 & $<.1$ & $<1$ & $<1$ & $<1$ & -- & .9 \\
\hline $09-18-84$ & .4 & -- & 5 & -- & $<.1$ & -- & $<1$ & $<1$ & -- & 1.5 \\
\hline $09-25-84$ & .8 & -- & 5 & -- & $<.1$ & -- & $<1$ & $<1$ & -- & 1.6 \\
\hline $10-02-84$ & .6 & -- & 33 & -- & $<.1$ & -- & $<1$ & $<1$ & -- & 10 \\
\hline $10-09-84$ & .6 & - & 10 & -- & $<.1$ & -- & $<1$ & $<1$ & -- & -- \\
\hline $10-16-84$ & .1 & -- & 8 & -- & $<.1$ & -- & $<1$ & $<1$ & -- & 2.2 \\
\hline $10-23-84$ & .1 & -- & 16 & -- & $<.1$ & -- & $<1$ & $<1$ & -- & 4.9 \\
\hline $10-30-84$ & -- & -- & -- & -- & -- & -- & -- & -- & -- & -- \\
\hline $11-06-84$ & -- & -- & -- & -- & -- & -- & -- & -- & -- & -- \\
\hline $11-13-84$ & .6 & -- & 64 & -- & $<.1$ & -- & $<1$ & $<1$ & -- & 6.1 \\
\hline $11-20-84$ & .3 & 30 & 29 & $<.1$ & -- & $<1$ & $<1$ & $<1$ & -- & 3.7 \\
\hline $11-27-84$ & .1 & -- & 19 & -- & -- & -- & $<1$ & $<1$ & -- & 2.9 \\
\hline $12-05-84$ & .2 & -- & 20 & - & -- & -- & $<1$ & $<1$ & 2.7 & -- \\
\hline $12-11-84$ & 2.6 & -- & 17 & -- & .2 & -- & $<1$ & $<1$ & -- & 2.2 \\
\hline $12-18-84$ & .1 & 30 & 32 & -- & -- & -- & -- & -- & -- & 2.7 \\
\hline $12-26-84$ & .7 & -- & 37 & -- & -- & -- & -- & -- & -- & -- \\
\hline $01-02-85$ & .4 & -- & 40 & -- & -- & -- & -- & -- & -- & -- \\
\hline $01-08-85$ & .9 & -- & 25 & -- & -- & -- & -- & -- & -- & 2.3 \\
\hline $01-16-85$ & 1.0 & -- & 20 & -- & -- & -- & -- & -- & -- & 2.2 \\
\hline $01-22-85$ & .8 & -- & 17 & -- & -- & -- & -- & -- & -- & 2.8 \\
\hline $01-29-85$ & .1 & -- & 13 & -- & -- & -- & -- & -- & -- & 1.5 \\
\hline $02-05-85$ & .1 & -- & 11 & -- & -- & -- & -- & -- & -- & 2.1 \\
\hline $02-13-85$ & .5 & -- & 39 & -- & -- & -- & -- & - & -- & 2.7 \\
\hline $02-19-85$ & .2 & -- & 28 & -- & -- & -- & -- & -- & -- & 2.1 \\
\hline $02-25-85$ & .1 & -- & 62 & -- & -- & -- & -- & -- & -- & 3.6 \\
\hline $03-05-85$ & .1 & -- & 28 & -- & -- & -- & -- & -- & -- & 2.0 \\
\hline
\end{tabular}

See footnotes at end of table. 
Table 3.--Physical characteristics and concentrations of chemical constituents of streamflow at station number 01174565, West Branch Swift River near Shutesbury, Massachusetts--Continued

\begin{tabular}{|c|c|c|c|c|c|c|c|c|c|}
\hline Date & Time & $\begin{array}{l}\text { Stream- } \\
\text { flow, } \\
\text { instan- } \\
\text { taneous } \\
\left(\mathrm{ft}^{3} / \mathrm{s}\right)\end{array}$ & $\begin{array}{l}\text { Spe- } \\
\text { cific } \\
\text { con- } \\
\text { duct- } \\
\text { ance } \\
(\mu \mathrm{s} / \mathrm{cm})\end{array}$ & $\begin{array}{c}\mathrm{pH} \\
\text { (stand- } \\
\text { ard } \\
\text { units) }\end{array}$ & $\begin{array}{l}\text { Temper- } \\
\text { ature } \\
\left(^{\circ} \mathrm{C}\right)\end{array}$ & $\begin{array}{c}\text { Hard- } \\
\text { ness } \\
(\mathrm{mg} / \mathrm{L} \\
\mathrm{as} \\
\left.\mathrm{CaCO}_{3}\right)\end{array}$ & $\begin{array}{l}\text { Calcium, } \\
\text { dis- } \\
\text { solved } \\
\text { (mg/L } \\
\text { as Ca) }\end{array}$ & $\begin{array}{l}\text { Magne- } \\
\text { sium, } \\
\text { dis- } \\
\text { solved } \\
\text { (mg/L } \\
\text { as Mg) }\end{array}$ & $\begin{array}{l}\text { Sodium, } \\
\text { dis- } \\
\text { solved } \\
\text { (mg/I } \\
\text { as } \mathrm{Na} \text { ) }\end{array}$ \\
\hline $\begin{array}{l}03-12-85 \\
03-19-85 \\
03-26-85 \\
04-02-85 \\
04-09-85\end{array}$ & $\begin{array}{l}09: 45 \\
10: 30 \\
16: 00 \\
13: 30 \\
13: 00\end{array}$ & $\begin{array}{r}100 \\
34 \\
20 \\
23 \\
26\end{array}$ & $\begin{array}{l}27 \\
33 \\
34 \\
33 \\
35\end{array}$ & $\begin{array}{l}5.4 \\
6.2 \\
6.4 \\
6.3 \\
6.2\end{array}$ & $\begin{array}{r}2.0 \\
.5 \\
4.0 \\
4.0 \\
6.0\end{array}$ & $\begin{array}{r}6 \\
7 \\
-- \\
7 \\
7\end{array}$ & $\begin{array}{r}1.7 \\
2.2 \\
-- \\
2.1 \\
2.2\end{array}$ & $\begin{array}{r}0.37 \\
. .44 \\
-- \\
.42 \\
.44\end{array}$ & $\begin{array}{r}1.9 \\
2.6 \\
-- \\
2.7 \\
3.0\end{array}$ \\
\hline $\begin{array}{l}04-16-85 \\
04-23-85 \\
04-30-85 \\
05-07-85 \\
05-14-85\end{array}$ & $\begin{array}{l}11: 30 \\
14: 30 \\
13: 15 \\
12: 30 \\
13: 15\end{array}$ & $\begin{array}{c}14 \\
10 \\
8.6 \\
14 \\
7.4\end{array}$ & $\begin{array}{l}37 \\
38 \\
-- \\
37 \\
43\end{array}$ & $\begin{array}{l}6.4 \\
6.5 \\
6.6 \\
6.5 \\
6.6\end{array}$ & $\begin{array}{r}9.5 \\
12.0 \\
12.0 \\
10.0 \\
17.0\end{array}$ & $\begin{array}{l}8 \\
8 \\
9 \\
5 \\
9\end{array}$ & $\begin{array}{l}2.4 \\
2.4 \\
2.7 \\
1.5 \\
2.6\end{array}$ & $\begin{array}{l}.51 \\
.53 \\
.46 \\
.31 \\
.52\end{array}$ & $\begin{array}{l}3.2 \\
3.5 \\
3.8 \\
2.1 \\
3.8\end{array}$ \\
\hline $\begin{array}{l}05-21-85 \\
05-28-85 \\
06-04-85^{1} \\
06-11-85^{1} \\
06-18-85\end{array}$ & $\begin{array}{l}12: 30 \\
13: 15 \\
13: 30 \\
14: 00 \\
13: 00\end{array}$ & $\begin{array}{l}11 \\
8.6 \\
5.6 \\
4.1 \\
15\end{array}$ & $\begin{array}{l}34 \\
41 \\
41 \\
45 \\
41\end{array}$ & $\begin{array}{l}6.4 \\
6.4 \\
6.6 \\
6.1 \\
6.6\end{array}$ & $\begin{array}{l}14.0 \\
14.5 \\
15.5 \\
16.0 \\
15.5\end{array}$ & $\begin{array}{r}-8 \\
-9 \\
-\frac{9}{9}\end{array}$ & $\begin{array}{c}2.4 \\
-2.7 \\
-- \\
2.6\end{array}$ & 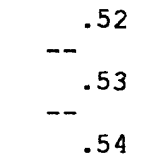 & $\begin{array}{c}3.1 \\
-\overline{3.8} \\
-- \\
3.7\end{array}$ \\
\hline $\begin{array}{l}06-25-85 \\
07-02-85 \\
07-10-85^{1} \\
07-16-85 \\
07-23-85\end{array}$ & $\begin{array}{l}14: 30 \\
13: 30 \\
13: 00 \\
13: 30 \\
14: 00\end{array}$ & $\begin{array}{l}5.5 \\
4.5 \\
2.6 \\
2.5 \\
1.1\end{array}$ & $\begin{array}{l}46 \\
39 \\
47 \\
45 \\
56\end{array}$ & $\begin{array}{l}6.6 \\
6.5 \\
6.6 \\
6.8 \\
6.8\end{array}$ & $\begin{array}{l}14.5 \\
-- \\
20.0 \\
-- \\
17.0\end{array}$ & $\begin{array}{r}-- \\
-- \\
--\end{array}$ & $\begin{array}{c}-- \\
-\overline{-} .7 \\
--\end{array}$ & $\begin{array}{l}-.53 \\
-. .59\end{array}$ & $\begin{array}{c}-- \\
-- \\
-- \\
--\end{array}$ \\
\hline $\begin{array}{l}07-30-85^{1} \\
07-30-85^{1} \\
08-07-85 \\
08-13-85 \\
08-20-85\end{array}$ & $\begin{array}{l}12: 15 \\
13: 15 \\
10: 45 \\
12: 30 \\
16: 00\end{array}$ & $\begin{array}{c}2.6 \\
-- \\
2.6 \\
1.7 \\
5.2\end{array}$ & $\begin{array}{l}50 \\
-- \\
48 \\
51 \\
39\end{array}$ & $\begin{array}{c}6.7 \\
-- \\
6.6 \\
6.8 \\
6.3\end{array}$ & $\begin{array}{l}17.0 \\
-- \\
15.5 \\
15.0 \\
19.0\end{array}$ & $\begin{array}{r}11 \\
9 \\
-- \\
10 \\
--\end{array}$ & $\begin{array}{c}3.5 \\
2.7 \\
-- \\
3.2 \\
--\end{array}$ & $\begin{array}{r}.60 \\
-.46 \\
-.\end{array}$ & $\begin{array}{r}5.1 \\
3.8 \\
-- \\
4.6 \\
--\end{array}$ \\
\hline $\begin{array}{l}08-26-85 \\
09-04-85 \\
09-10-85\end{array}$ & $\begin{array}{l}15: 45 \\
11: 45 \\
14: 45\end{array}$ & $\begin{array}{l}20 \\
6.2 \\
--\end{array}$ & $\begin{array}{l}32 \\
36 \\
32\end{array}$ & $\begin{array}{l}6.0 \\
6.2 \\
6.1\end{array}$ & $\begin{array}{l}16.5 \\
16.0 \\
15.0\end{array}$ & $\begin{array}{r}7 \\
-- \\
--\end{array}$ & $\begin{array}{l}2.1 \\
-- \\
--\end{array}$ & $--^{.40}$ & $\begin{array}{l}2.3 \\
-- \\
--\end{array}$ \\
\hline
\end{tabular}


Table 3.--Physical characteristics and concentrations of chemical constituents of streamflow at station number 01174565, West Branch Swift River near Shutesbury, Massachusetts--Continued

\begin{tabular}{|c|c|c|c|c|c|c|c|c|c|c|}
\hline Date & $\begin{array}{l}\text { Potas- } \\
\text { sium, } \\
\text { dis- } \\
\text { solved } \\
\text { (mg/L } \\
\text { as K) }\end{array}$ & $\begin{array}{l}\text { Nitro- } \\
\text { gen, } \\
\text { ammonia } \\
\text { total } \\
\text { (mg/L } \\
\text { as N) }\end{array}$ & $\begin{array}{c}\text { Nitro- } \\
\text { gen, } \\
\text { ammonia, } \\
\text { dis- } \\
\text { solved } \\
\text { (mg/L } \\
\text { as N) }\end{array}$ & $\begin{array}{c}\text { Alka- } \\
\text { linity } \\
\text { field } \\
\text { (mg/L } \\
\text { as } \\
\mathrm{CaCO}_{3} \text { ) }\end{array}$ & $\begin{array}{l}\text { Sulfate, } \\
\text { dis- } \\
\text { solved } \\
\text { (mg/L } \\
\text { as } \mathrm{SO}_{4} \text { ) }\end{array}$ & $\begin{array}{c}\text { Nitro- } \\
\text { gen, } \\
\text { nitrate, } \\
\text { dis- } \\
\text { solved } \\
\text { (mg/L } \\
\text { as N) }\end{array}$ & $\begin{array}{l}\text { Phos- } \\
\text { phorus, } \\
\text { ortho, } \\
\text { dis- } \\
\text { solved } \\
\text { (mg/L } \\
\text { as P) }\end{array}$ & $\begin{array}{l}\text { Chlo- } \\
\text { ride, } \\
\text { dis- } \\
\text { solved } \\
\text { (mg/L } \\
\text { as Cl) }\end{array}$ & $\begin{array}{l}\text { Fluo- } \\
\text { ride, } \\
\text { dis- } \\
\text { solved } \\
\text { (mg人L } \\
\text { as F) }\end{array}$ & $\begin{array}{l}\text { Silica, } \\
\text { dis- } \\
\text { solved } \\
\text { (mg/L } \\
\text { as } \\
\mathrm{SiO}_{2} \text { ) }\end{array}$ \\
\hline $\begin{array}{l}03-12-85 \\
03-19-85 \\
03-26-85 \\
04-02-85 \\
04-09-85\end{array}$ & $\begin{array}{r}0.59 \\
-.40 \\
-\quad \\
.37 \\
.39\end{array}$ & $\begin{array}{l}-- \\
-- \\
-- \\
-- \\
--\end{array}$ & $\begin{array}{l}0.13 \\
<.01 \\
<.01 \\
.27 \\
.08\end{array}$ & $\begin{array}{l}0.4 \\
1.4 \\
1.4 \\
1.0 \\
1.2\end{array}$ & $\begin{array}{r}8.0 \\
8.0 \\
-\overline{4} .2 \\
7.3\end{array}$ & $\begin{array}{r}0.12 \\
-.02 \\
-\quad \\
.02 \\
.03\end{array}$ & $\begin{array}{r}<0.01 \\
<.01 \\
-- \\
.03 \\
<.01\end{array}$ & $\begin{array}{c}2.6 \\
-.86 \\
-4.22 \\
4.1\end{array}$ & $\begin{array}{r}0.06 \\
-.04 \\
-\quad \\
.03 \\
.05\end{array}$ & $\begin{array}{r}4.7 \\
7.3 \\
-- \\
6.4 \\
6.3\end{array}$ \\
\hline $\begin{array}{l}04-16-85 \\
04-23-85 \\
04-30-85 \\
05-07-85 \\
05-14-85\end{array}$ & $\begin{array}{l}.42 \\
.45 \\
.47 \\
.32 \\
.50\end{array}$ & $\begin{array}{l}-- \\
-- \\
-- \\
-- \\
--\end{array}$ & $\begin{array}{r}.01 \\
.03 \\
<.01 \\
<.01 \\
.05\end{array}$ & $\begin{array}{l}1.8 \\
2.3 \\
2.9 \\
1.8 \\
2.9\end{array}$ & $\begin{array}{l}6.6 \\
6.7 \\
6.9 \\
4.2 \\
6.6\end{array}$ & $\begin{array}{l}.02 \\
.02 \\
.03 \\
.03 \\
.02\end{array}$ & $\begin{array}{l}<.01 \\
<.01 \\
<.01 \\
<.01 \\
<.01\end{array}$ & $\begin{array}{l}3.7 \\
4.2 \\
4.5 \\
2.2 \\
4.8\end{array}$ & $\begin{array}{l}.07 \\
.05 \\
.06 \\
.06 \\
.06\end{array}$ & $\begin{array}{l}6.1 \\
5.5 \\
5.2 \\
3.3 \\
4.8\end{array}$ \\
\hline $\begin{array}{l}05-21-85 \\
05-28-85 \\
06-04-85 \\
06-11-85 \\
06-18-85\end{array}$ & $\begin{array}{r}-.40 \\
-.44 \\
-.45\end{array}$ & $\begin{array}{l}-- \\
-- \\
-- \\
-- \\
--\end{array}$ & $\begin{array}{c}<.01 \\
-- \\
-.01 \\
- \\
<.01\end{array}$ & $\begin{array}{l}1.8 \\
2.9 \\
2.9 \\
3.7 \\
2.9\end{array}$ & $\begin{array}{c}6.4 \\
-- \\
6.1 \\
-- \\
6.6\end{array}$ & $\begin{array}{r}-.02 \\
-\quad .01 \\
- \\
-.04\end{array}$ & $\begin{array}{l}-.01 \\
--.01 \\
-- \\
<.01\end{array}$ & $\begin{array}{c}3.8 \\
-- \\
5.4 \\
-- \\
5.4\end{array}$ & $\begin{array}{r}-.08 \\
-.06\end{array}$ & $\begin{array}{l}4.8 \\
-- \\
5.8 \\
-- \\
6.0\end{array}$ \\
\hline $\begin{array}{l}06-25-85 \\
07-02-85 \\
07-10-85 \\
07-16-85 \\
07-23-85\end{array}$ & --.44 & $\begin{array}{l}-- \\
-- \\
-- \\
-- \\
--\end{array}$ & $\begin{array}{l}-- \\
<.01 \\
-- \\
<.01 \\
--\end{array}$ & $\begin{array}{l}4.1 \\
2.9 \\
4.9 \\
4.6 \\
5.5\end{array}$ & $\begin{array}{c}-- \\
49 \\
-- \\
5.5 \\
--\end{array}$ & $\begin{array}{c}-. \\
-.04 \\
\ldots\end{array}$ & $\begin{array}{l}-- \\
<.01 \\
-- \\
<.01 \\
--\end{array}$ & $\begin{array}{c}-- \\
6.4 \\
-- \\
--\end{array}$ & $-_{--}^{-.04}$ & $\begin{array}{c}-- \\
6.2 \\
-- \\
--\end{array}$ \\
\hline $\begin{array}{l}07-30-85 \\
07-30-85 \\
08-07-85 \\
08-13-85 \\
08-20-85\end{array}$ & $\begin{array}{r}.56 \\
-.47 \\
-. \\
-\end{array}$ & $\begin{array}{l}-- \\
-- \\
-- \\
-- \\
--\end{array}$ & $\begin{array}{r}<.06 \\
<-.01 \\
-.02\end{array}$ & $\begin{array}{c}4.9 \\
-- \\
3.7 \\
4.5 \\
2.5\end{array}$ & $\begin{array}{c}6.6 \\
6.9 \\
-- \\
5.5 \\
--\end{array}$ & $\begin{array}{r}.08 \\
-.03 \\
\ldots-\end{array}$ & $\begin{array}{l}<.01 \\
<.01 \\
-- \\
<.01 \\
--\end{array}$ & $\begin{array}{c}6.7 \\
4.5 \\
-- \\
5.6 \\
--\end{array}$ & $\begin{array}{r}.09 \\
-.06 \\
--.07\end{array}$ & $\begin{array}{c}6.7 \\
5.2 \\
-- \\
6.5 \\
--\end{array}$ \\
\hline $\begin{array}{l}08-26-85 \\
09-04-85 \\
09-10-85\end{array}$ & $\begin{array}{l}.27 \\
--\end{array}$ & $\begin{array}{l}-- \\
-- \\
--\end{array}$ & $\begin{array}{l}<.01 \\
-- \\
--\end{array}$ & $\begin{array}{r}1.0 \\
-- \\
.6\end{array}$ & $\begin{array}{l}6.1 \\
-- \\
--\end{array}$ & --.01 & $\begin{array}{l}<.01 \\
-- \\
--\end{array}$ & $\begin{array}{l}2.3 \\
-- \\
--\end{array}$ & --.08 & $\begin{array}{l}6.9 \\
-- \\
--\end{array}$ \\
\hline
\end{tabular}

See footnotes at end of table. 
Table 3.--Physical characteristics and concentrations of chemical constituents of streamflow at station number 01174565, West Branch Swift River near Shutesbury, Massachusetts--Continued

\begin{tabular}{|c|c|c|c|c|c|c|c|c|c|c|}
\hline Date & $\begin{array}{l}\text { Solids, } \\
\text { sum of } \\
\text { consti- } \\
\text { tuents, } \\
\text { dis- } \\
\text { solved } \\
\text { (mg/L) }\end{array}$ & $\begin{array}{l}\text { Alum- } \\
\text { inum, } \\
\text { total } \\
\text { recov- } \\
\text { erable } \\
\text { ( } \mu \mathrm{g} / \mathrm{L} \\
\text { as Al) }\end{array}$ & $\begin{array}{l}\text { Alum- } \\
\text { inum, } \\
\text { dis- } \\
\text { solved } \\
(\mu \mathrm{g} / \mathrm{L} \\
\text { as Al) }\end{array}$ & $\begin{array}{c}\text { Arsenic, } \\
\text { dis- } \\
\text { solved } \\
\text { ( } \mu \text { g/L } \\
\text { as As) }\end{array}$ & $\begin{array}{c}\text { Arsenic, } \\
\text { total } \\
(\mu \mathrm{g} / \mathrm{L} \\
\text { as As) }\end{array}$ & $\begin{array}{c}\text { Copper, } \\
\text { total } \\
\text { recov- } \\
\text { erable } \\
(\mu g / L \\
\text { as } \mathrm{Cu})\end{array}$ & $\begin{array}{l}\text { Copper, } \\
\text { dis- } \\
\text { solved } \\
\text { ( } \mu \mathrm{g} / \mathrm{L} \\
\text { as } \mathrm{Cu})\end{array}$ & $\begin{array}{l}\text { Iron, } \\
\text { total } \\
\text { recov- } \\
\text { erable } \\
(\mu g / L \\
\text { as Fe) }\end{array}$ & $\begin{array}{l}\text { Iron, } \\
\text { dis- } \\
\text { solved } \\
\text { ( } \mu \text { g/L } \\
\text { as } \mathrm{Fe})\end{array}$ & $\begin{array}{l}\text { Lead, } \\
\text { total } \\
\text { recov- } \\
\text { erable } \\
(\mu g / L \\
\text { as } \mathrm{Pb})\end{array}$ \\
\hline $03-12-85$ & 21 & -- & ${ }^{2} 260$ & -- & -- & -- & 1.8 & -- & 51 & -- \\
\hline $03-19-85$ & 23 & -- & $2<10$ & -- & -- & -- & .6 & -- & 24 & -- \\
\hline $03-26-85$ & -- & -- & ${ }^{2} 70$ & -- & -- & -- & 1.0 & -- & -- & -- \\
\hline $04-02-85$ & 14 & -- & 290 & -- & -- & -- & .8 & -- & 21 & -- \\
\hline $04-09-85$ & 25 & -- & ${ }^{2} 90$ & -- & -- & -- & .1 & -- & 24 & -- \\
\hline $04-16-85$ & 24 & -- & ${ }^{2} 60$ & -- & -- & -- & .4 & -- & 27 & -- \\
\hline $04-23-85$ & 25 & -- & ${ }^{2} 70$ & -- & -- & -- & .1 & -- & 35 & -- \\
\hline $04-30-85$ & 26 & -- & $2^{--}$ & -- & -- & -- & -- & -- & 34 & -- . \\
\hline $05-07-85$ & 15 & -- & 280 & -- & -- & -- & $<.1$ & -- & 25 & -- \\
\hline $05-14-85$ & 26 & -- & ${ }^{2} 50$ & -- & -- & -- & $<.1$ & -- & 47 & -- \\
\hline $05-21-85$ & 23 & -- & ${ }^{2} 100$ & -- & -- & -- & 13.0 & -- & 44 & -- \\
\hline $05-28-85$ & -- & -- & $2^{--}$ & -- & -- & $-\cdot$ & -- & -- & -- & -- \\
\hline $06-04-85$ & 27 & -- & ${ }^{2} 60$ & -- & -- & -- & $<.1$ & -- & 52 & -- \\
\hline $06-11-85$ & -- & -- & $2^{--}$ & -- & -- & -- & -- & -- & -- & -- \\
\hline $06-18-85$ & 27 & -- & 250 & -- & -- & -- & $<.1$ & -- & 67 & -- \\
\hline $06-25-85$ & -- & -- & $2^{--}$ & -- & -- & -- & -- & -- & -- & -- \\
\hline $07-02-85$ & 41 & -- & ${ }^{2} 40$ & -- & -- & -- & .1 & -- & 74 & -- \\
\hline $07-10-85$ & -- & -- & $2^{--}$ & -- & -- & -- & -- & -- & -- & -- \\
\hline $07-16-85$ & 30 & -- & ${ }^{2} 30$ & -- & -- & -- & $<.1$ & -- & 110 & -- \\
\hline $07-23-85$ & -- & -- & -- & -- & -- & -- & -- & -- & -- & -- \\
\hline $07-30-85$ & 33 & -- & 270 & -- & -- & -- & 2.0 & -- & 98 & $=-$ \\
\hline $07-30-85$ & -- & -- & -- & -- & -- & -- & 1.0 & -- & 34 & -- \\
\hline $08-07-85$ & -- & -- & $2^{--}$ & -- & -- & -- & -- & -- & -- & -- \\
\hline $08-13-85$ & 30 & -- & 230 & -- & -- & -- & 1.0 & -- & 86 & -- \\
\hline $08-20-85$ & -- & -- & -- & -- & -- & -- & -- & -- & -- & -- \\
\hline $08-26-85$ & 21 & -- & $2_{50}$ & -- & -- & -- & $<.1$ & -- & 10,0 & -- \\
\hline $09-04-85$ & -- & -- & -- & -- & -- & -- & -- & -- & -- & -- \\
\hline $09-10-85$ & -- & -- & -- & -- & -- & -- & -- & -- & -- & -- \\
\hline \multicolumn{11}{|c|}{ See footnotes at end of table. } \\
\hline
\end{tabular}


Table 3.--Physical characteristics and concentrations of chemical constituents of streamflow at station number 01174565, West Branch Swift River near Shutesbury, Massachusetts--Continued

\begin{tabular}{|c|c|c|c|c|c|c|c|c|c|c|}
\hline Date & $\begin{array}{l}\text { Lead, } \\
\text { dis- } \\
\text { solved } \\
(\mu \mathrm{g} / \mathrm{L} \\
\text { as } \mathrm{Pb})\end{array}$ & $\begin{array}{l}\text { Manga- } \\
\text { nese, } \\
\text { total } \\
\text { recov- } \\
\text { erable } \\
\text { ( } \mu \text { g } / L \\
\text { as } M n)\end{array}$ & $\begin{array}{l}\text { Manga- } \\
\text { nese, } \\
\text { dis- } \\
\text { solved } \\
(\mu g / L \\
\text { as } M n)\end{array}$ & $\begin{array}{c}\text { Mercury, } \\
\text { total } \\
\text { recov- } \\
\text { erable } \\
\text { ( } \mu \mathrm{g} / \mathrm{L} \\
\text { as } \mathrm{Hg})\end{array}$ & $\begin{array}{l}\text { Mercury, } \\
\text { dis- } \\
\text { solved } \\
(\mu g / L \\
\text { as } \mathrm{Hg})\end{array}$ & $\begin{array}{l}\text { Sele- } \\
\text { nium, } \\
\text { total } \\
\text { ( } \mu \mathrm{g} / \mathrm{L} \\
\text { as } \mathrm{Se})\end{array}$ & $\begin{array}{l}\text { Sele- } \\
\text { nium, } \\
\text { dis- } \\
\text { solved } \\
\text { ( } \mu \text { g/L } \\
\text { as Se) }\end{array}$ & $\begin{array}{l}\text { Vana- } \\
\text { dium, } \\
\text { dis- } \\
\text { solved } \\
(\mu g / L \\
\text { as V) }\end{array}$ & $\begin{array}{c}\text { Carbon, } \\
\text { organic } \\
\text { total } \\
\text { (mg/L } \\
\text { as C) }\end{array}$ & $\begin{array}{c}\text { Carbon, } \\
\text { organic, } \\
\text { dis- } \\
\text { solved } \\
\text { (mg/L } \\
\text { as C) }\end{array}$ \\
\hline $03-12-85$ & 0.8 & -- & 99 & -- & -- & -- & -- & -- & -- & -- \\
\hline $03-19-85$ & .5 & -- & 36 & -- & -- & -- & -- & -- & -- & 2.8 \\
\hline $03-26-85$ & $<.1$ & -- & -- & -- & -- & -- & -- & -- & -- & 2.5 \\
\hline $04-02-85$ & .4 & -- & 21 & -- & -- & -- & -- & -- & -- & 2.9 \\
\hline $04-09-85$ & .3 & -- & 21 & -- & -- & -- & -- & -- & -- & 2.8 \\
\hline $04-16-85$ & .5 & -- & 14 & -- & -- & -- & -- & -- & -- & 2.8 \\
\hline $04-23-85$ & .2 & -- & 11 & -- & -- & -- & -- & -- & -- & 2.7 \\
\hline $04-30-85$ & -- & -- & -- & -- & - & -- & -- & -- & -- & 2.3 \\
\hline $05-07-85$ & $<.1$ & -- & 8 & - & -- & -- & -- & -- & -- & 3.1 \\
\hline $05-14-85$ & 1.0 & -- & 8 & -- & -- & -- & -- & -- & -- & 3.0 \\
\hline $05-21-85$ & 4.0 & -- & 13 & -- & -- & -- & -- & -- & -- & 3.0 \\
\hline $05-28-85$ & -- & -- & -- & -- & -- & -- & -- & -- & -- & -- \\
\hline $06-04-85$ & $<.1$ & -- & 7 & -- & -- & -- & -- & -- & -- & 2.9 \\
\hline $06-11-85$ & -- & -- & -- & -- & -- & -- & -- & -- & -- & -- \\
\hline $06-18-85$ & $<.1$ & -- & 8 & -- & -- & -- & -- & -- & -- & 2.4 \\
\hline $06-25-85$ & -- & -- & -- & -- & -- & -- & -- & -- & -- & -- \\
\hline $07-02-85$ & 1.2 & -- & 7 & -- & -- & -- & -- & -- & -- & -- \\
\hline $07-10-85$ & -- & -- & -- & -- & -- & -- & -- & -- & -- & - \\
\hline $07-16-85$ & $<.1$ & -- & 6 & -- & -- & -- & -- & -- & -- & 2.7 \\
\hline $07-23-85$ & -- & -- & -- & -- & -- & -- & -- & -- & -- & -- \\
\hline $07-30-85$ & $<.1$ & -- & 5 & -- & -- & -- & -- & -- & -- & -- \\
\hline $07-30-85$ & $<.1$ & -- & 7 & -- & -- & -- & -- & -- & -- & 2.3 \\
\hline $08-07-85$ & -- & -- & -- & -- & -- & -- & -- & -- & -- & -- \\
\hline $08-13-85$ & $<.1$ & -- & 8 & -- & -- & -- & -- & -- & -- & 2.5 \\
\hline $08-20-85$ & -- & -- & -- & -- & -- & -- & -- & -- & -- & -- \\
\hline $08-26-85$ & $<.1$ & -- & 28 & -- & -- & -- & -- & -- & -- & -- \\
\hline $09-04-85$ & -- & -- & -- & -- & -- & -- & -- & -- & -- & -- \\
\hline $09-10-85$ & -- & -- & -- & -- & -- & -- & -- & -- & -- & -- \\
\hline
\end{tabular}

1 Analysis was used in calculation of volume-weighted base flow stream chemistry.

2 Sample filtered through 0.1 micrometer pore filter.

3 Value omitted from the data set for regression analysis because it was spurious in plot (Note: $r^{2}$ went from 0.32 to 0.62 when these two points were omitted).

4 Anomalous value that contributed to cation-anion balance difference of greater than 10 percent; value discarded in data analysis. 
Table 4.--Physical characteristics and concentrations of chemical constituents of streamflow at station number 01174555, West Branch Swift River near Cooleyville, Massachusetts

$\left[\mathrm{ft}^{3} / \mathrm{s}\right.$, cubic feet per second; $\mu \mathrm{S} / \mathrm{cm}$, microsiemens per centimeter, ${ }^{\circ} \mathrm{C}$, degrees Celsius; $\mathrm{mg} / \mathrm{L}$, milligrams per liter, $\mu \mathrm{g} / \mathrm{L}$, micrograms per liter; $<$, less than; dashes indicate not analyzed]

\begin{tabular}{|c|c|c|c|c|c|c|c|c|c|}
\hline Date & Time & $\begin{array}{l}\text { Stream- } \\
\text { flow, } \\
\text { instan- } \\
\text { taneous } \\
\left(\mathrm{ft}^{3} / \mathrm{s}\right)\end{array}$ & $\begin{array}{l}\text { Spe- } \\
\text { cific } \\
\text { con- } \\
\text { duct- } \\
\text { ance } \\
(\mu s / \mathrm{cm})\end{array}$ & $\begin{array}{c}\mathrm{pH} \\
\text { (stand- } \\
\text { ard } \\
\text { units) }\end{array}$ & $\begin{array}{l}\text { Temper- } \\
\text { ature } \\
\left({ }^{\circ} \mathrm{C}\right)\end{array}$ & $\begin{array}{l}\text { Hard- } \\
\text { ness } \\
(\mathrm{mg} / \mathrm{L} \\
\mathrm{as} \\
\mathrm{CaCO}_{3}\end{array}$ & $\begin{array}{c}\text { Calcium, } \\
\text { dis- } \\
\text { solved } \\
\text { (mg/L } \\
\text { as Ca) }\end{array}$ & $\begin{array}{l}\text { Magne- } \\
\text { sium, } \\
\text { dis- } \\
\text { solved } \\
\text { (mg/L } \\
\text { as Mg) }\end{array}$ & $\begin{array}{l}\text { Sodium, } \\
\text { dis- } \\
\text { solved } \\
\text { (mg/L } \\
\text { as Na) }\end{array}$ \\
\hline $04-05-84$ & $15: 00$ & 126 & 26 & 5.0 & 1.5 & -- & -- & -- & -- \\
\hline $04-05-84^{1}$ & $15: 10$ & -- & -- & -- & -- & -- & -- & -- & -- \\
\hline $05-30-84$ & $14: 15$ & -- & 26 & 4.7 & 10.5 & 4 & 1.2 & 0.31 & 1.4 \\
\hline $05-30-84^{1}$ & $14: 20$ & -- & -- & -- & -- & -- & -- & -- & -- \\
\hline $08-28-84$ & $14: 15$ & -- & 21 & 5.7 & 19.0 & -- & -- & -- & -- \\
\hline $09-11-84$ & $16: 45$ & .06 & 21 & 5.7 & 18.5 & 4 & 1.2 & .18 & 2.1 \\
\hline $10-02-84$ & -- & -- & 33 & 5.0 & 9.0 & -- & -- & -- & -- \\
\hline $10-17-84$ & $09: 30$ & -- & 29 & 5.8 & 8.0 & -- & -- & -- & -- \\
\hline $12-18-84$ & $15: 45$ & 7.6 & 36 & 4.9 & 3.0 & 6 & 1.7 & .38 & 2.2 \\
\hline $04-30-85$ & $12: 15$ & -- & 30 & 5.1 & 14.5 & -- & -- & -- & -- \\
\hline $05-07-85$ & $14: 30$ & -- & 31 & 5.1 & 10.0 & -- & -- & -- & -- \\
\hline $05-28-85$ & $12: 30$ & -- & 28 & 5.1 & 15.0 & -- & -- & -- & -- \\
\hline $06-25-85$ & $13: 30$ & -- & 25 & 5.2 & 15.0 & -- & -- & -- & -- \\
\hline $07-23-85$ & $13: 00$ & -- & 25 & 5.3 & 17.0 & -- & -- & -- & -- \\
\hline $08-07-85$ & $12: 00$ & -- & 33 & 5.0 & 17.0 & -- & -- & -- & -- \\
\hline $08-26-85$ & $10: 15$ & 8.1 & 30 & 4.8 & 16.0 & 5 & 1.6 & .31 & 1.8 \\
\hline
\end{tabular}

See footnote at end of table.

\begin{tabular}{|c|c|c|c|c|c|c|c|c|c|c|}
\hline Date & $\begin{array}{l}\text { Potas- } \\
\text { sium, } \\
\text { dis- } \\
\text { solved } \\
\text { (mg/L } \\
\text { as K) }\end{array}$ & $\begin{array}{c}\text { Nitro- } \\
\text { gen, } \\
\text { ammonia } \\
\text { total } \\
\text { (mg/L } \\
\text { as N) }\end{array}$ & $\begin{array}{c}\text { Nitro- } \\
\text { gen, } \\
\text { ammonia, } \\
\text { dis- } \\
\text { solved } \\
\text { (mg/L } \\
\text { as N) }\end{array}$ & $\begin{array}{c}\text { Alka- } \\
\text { linity } \\
\text { field } \\
\text { (mg/L } \\
\text { as } \\
\mathrm{CaCO}_{3} \text { ) }\end{array}$ & $\begin{array}{l}\text { Sulfate, } \\
\text { dis- } \\
\text { solved } \\
(\mathrm{mg} / \mathrm{L} \\
\left.\text { as } \mathrm{SO}_{4}\right)\end{array}$ & $\begin{array}{c}\text { Nitro- } \\
\text { gen, } \\
\text { nitrate, } \\
\text { dis- } \\
\text { solved } \\
\text { (mg/L } \\
\text { as } N \text { ) }\end{array}$ & $\begin{array}{l}\text { Phos- } \\
\text { phorus, } \\
\text { ortho, } \\
\text { dis- } \\
\text { solved } \\
\text { (mg/L } \\
\text { as P) }\end{array}$ & $\begin{array}{l}\text { Chlo- } \\
\text { ride, } \\
\text { dis- } \\
\text { solved } \\
\text { (mg/L } \\
\text { as Cl) }\end{array}$ & $\begin{array}{l}\text { Fluo- } \\
\text { ride, } \\
\text { dis- } \\
\text { solved } \\
\text { (mg/L } \\
\text { as F) }\end{array}$ & $\begin{array}{l}\text { silica, } \\
\text { dis- } \\
\text { solved } \\
\text { (mg/L } \\
\text { as } \\
\mathrm{SiO}_{2} \text { ) }\end{array}$ \\
\hline $04-05-84$ & -- & $<0.001$ & -- & 0.0 & -- & -- & -- & -- & -- & -- \\
\hline $04-05-84$ & -- & -- & -- & -- & -- & -- & -- & -- & -- & -- \\
\hline $05-30-84$ & 0.26 & .073 & -- & .0 & 5.1 & -- & -- & 1.6 & 0.05 & 4.2 \\
\hline $05-30-84$ & -- & -- & -- & -- & -- & -- & -- & -- & -- & -- \\
\hline $08-28-84$ & -- & -- & -- & .2 & -- & -- & -- & -- & -- & -- \\
\hline $09-11-84$ & .42 & .117 & -- & .8 & 4.4 & -- & -- & 2.1 & .07 & 4.7 \\
\hline $10-02-84$ & -- & -- & -- & -- & -- & -- & -- & -- & -- & -- \\
\hline $10-17-84$ & -- & -- & -- & .6 & -- & -- & -- & -- & -- & -- \\
\hline $12-18-84$ & .30 & .008 & -- & .0 & -- & 0.28 & -- & 2.7 & .37 & 8.0 \\
\hline $04-30-85$ & -- & -- & -- & .3 & -- & -- & -- & -- & -- & -- \\
\hline $05-07-85$ & -- & -- & -- & .0 & -- & $\cdots$ & -- & -- & -- & -- \\
\hline $05-28-85$ & -- & -- & -- & .0 & -- & -- & -- & -- & -- & -- \\
\hline $06-25-85$ & -- & -- & -- & .1 & -- & -- & -- & -- & -- & -- \\
\hline $07-23-85$ & -- & -- & -- & -- & -- & -- & -- & -- & -- & -- \\
\hline $08-07-85$ & -- & -- & -- & .0 & -- & -- & -- & -- & -- & - \\
\hline $08-26-85$ & .16 & -- & $<0.01$ & .0 & 5.5 & .01 & $<0.01$ & 1.4 & .06 & 6.7 \\
\hline
\end{tabular}




\begin{tabular}{|c|c|c|c|c|c|c|c|c|c|c|}
\hline Date & $\begin{array}{l}\text { Solids, } \\
\text { sum of } \\
\text { constit- } \\
\text { uents, } \\
\text { dis- } \\
\text { solved } \\
(\mathrm{mg} / \mathrm{L})\end{array}$ & $\begin{array}{l}\text { Alum- } \\
\text { inum, } \\
\text { total } \\
\text { recov- } \\
\text { erable } \\
(\mu \mathrm{g} / \mathrm{L} \\
\text { as Al) }\end{array}$ & $\begin{array}{l}\text { Alum- } \\
\text { inum, } \\
\text { dis- } \\
\text { solved } \\
(\mu g / L \\
\text { as AI) }\end{array}$ & $\begin{array}{c}\text { Arsenic, } \\
\text { dis- } \\
\text { solved } \\
\text { ( } \mu \text { g/L } \\
\text { as As) }\end{array}$ & $\begin{array}{c}\text { Arsenic, } \\
\text { total } \\
\text { ( } \mu \text { g/L } \\
\text { as As) }\end{array}$ & $\begin{array}{c}\text { Copper, } \\
\text { total } \\
\text { recov- } \\
\text { erable } \\
(\mu g / L \\
\text { as } \mathrm{Cu})\end{array}$ & $\begin{array}{l}\text { Copper, } \\
\text { dis- } \\
\text { solved } \\
(\mu \mathrm{g} / \mathrm{L} \\
\text { as } \mathrm{Cu})\end{array}$ & $\begin{array}{l}\text { Iron, } \\
\text { total } \\
\text { recov- } \\
\text { erable } \\
(\mu \mathrm{g} / \mathrm{L} \\
\text { as } \mathrm{Fe})\end{array}$ & $\begin{array}{l}\text { Iron, } \\
\text { dis- } \\
\text { solved } \\
(\mu \mathrm{g} / \mathrm{L} \\
\text { as } \mathrm{Fe})\end{array}$ & $\begin{array}{l}\text { Lead, } \\
\text { total } \\
\text { recov- } \\
\text { erable } \\
(\mu g / L \\
\text { as } \mathrm{Pb})\end{array}$ \\
\hline $04-05-84$ & -- & -- & -- & -- & -- & -- & -- & -- &,-- & -- \\
\hline $04-05-84$ & -- & -- & ${ }^{1} 150$ & -- & -- & -- & -- & -- & ${ }^{1} 41$ & -- \\
\hline $05-30-84$ & 14 & -- & ,- & -- & -- & -- & -- & -- & 89 & -- \\
\hline $05-30-84$ & -- & -- & ${ }^{1} 290$ & -- & -- & -- & -- & -- & 193 & -- \\
\hline $08-28-84$ & -- & -- & -- & -- & -- & -- & -- & -- & -- & -- \\
\hline $09-11-84$ & -- & 90 & 80 & $<1$ & $<1$ & 1 & 1.6 & 460 & 200 & 1 \\
\hline $10-02-84$ & -- & - & -- & -- & -- & -- & - & -- & - & -- \\
\hline $10-17-84$ & -- & -- & -- & -- & -- & -- & -- & -- & -- & -- \\
\hline $12-18-84$ & -- & 200 & 180 & -- & -- & $<1$ & 1.8 & 200 & 62 & 2 \\
\hline $04-30-85$ & -- & -- & -- & -- & -- & -- & -- & -- & -- & -- \\
\hline $05-07-85$ & -- & -- & -- & -- & -- & -- & -- & -- & -- & -- \\
\hline $05-28-85$ & -- & -- & -- & -- & -- & -- & -- & -- & -- & -- \\
\hline $06-25-85$ & -- & -- & -- & -- & -- & -- & -- & -- & -- & -- \\
\hline $07-23-85$ & -- & -- & -- & -- & -- & -- & -- & -- & -- & -- \\
\hline $08-07-85$ & -- & -- & -- & -- & -- & -- & -- & -- & -- & -- \\
\hline $08-26-85$ & -- & -- & 260 & -- & -- & -- & -- & -- & 160 & -- \\
\hline
\end{tabular}

See footnote at end of table.

\begin{tabular}{|c|c|c|c|c|c|c|c|c|c|c|}
\hline Date & $\begin{array}{c}\text { Lead, } \\
\text { dis- } \\
\text { solved } \\
(\mu g / L \\
\text { as } \mathrm{Pb})\end{array}$ & $\begin{array}{l}\text { Manga- } \\
\text { nese, } \\
\text { total } \\
\text { recov- } \\
\text { erable } \\
(\mu g / L \\
\text { as } M n)\end{array}$ & $\begin{array}{l}\text { Manga- } \\
\text { nese, } \\
\text { dis- } \\
\text { solved } \\
\text { ( } \mu \text { g/L } \\
\text { as } M n)\end{array}$ & $\begin{array}{l}\text { Mercury, } \\
\text { total } \\
\text { recov- } \\
\text { erable } \\
(\mu g / L \\
\text { as } \mathrm{Hg})\end{array}$ & $\begin{array}{c}\text { Mercury, } \\
\text { dis- } \\
\text { solved } \\
(\mu g / L \\
\text { as Hg) }\end{array}$ & $\begin{array}{l}\text { Sele- } \\
\text { nium, } \\
\text { total } \\
(\mu g / L \\
\text { as Se) }\end{array}$ & $\begin{array}{l}\text { Sele- } \\
\text { nium, } \\
\text { dis- } \\
\text { solved } \\
\text { ( } \mu \text { g/L } \\
\text { as Se) }\end{array}$ & $\begin{array}{l}\text { Vana- } \\
\text { dium, } \\
\text { dis- } \\
\text { solved } \\
(\mu \mathrm{g} / \mathrm{L} \\
\text { as V) }\end{array}$ & $\begin{array}{c}\text { Carbon, } \\
\text { organic } \\
\text { total } \\
\text { (mg/L } \\
\text { as C) }\end{array}$ & $\begin{array}{c}\text { Carbon, } \\
\text { organic, } \\
\text { dis- } \\
\text { solved } \\
\text { (mg/L } \\
\text { as C) }\end{array}$ \\
\hline $04-05-84$ & -- & -- &,-- & -- & -- & -- & -- & -- & 3.5 & -- \\
\hline $04-05-84$ & -- & -- & ${ }^{1} 58$ & -- & -- & -- & -- & -- & -- & -- \\
\hline $05-30-84$ & -- & -- & 71 & -- & -- & -- & -- & -- & 5.7 & -- \\
\hline $05-30-84$ & -- & -- & ${ }^{1} 64$ & -- & -- & -- & -- & -- & -- & -- \\
\hline $08-28-84$ & -- & -- & -- & -- & -- & -- & -- & -- & -- & -- \\
\hline $09-11-84$ & 0.7 & 30 & 37 & $<0.1$ & 0.3 & $<1$ & $<1$ & $<1$ & -- & 4.3 \\
\hline $10-02-84$ & -- & -- & -- & -- & -- & -- & -- & -- & -- & - \\
\hline $10-17-84$ & -- & -- & -- & -- & -- & -- & -- & -- & -- & -- \\
\hline $12-18-84$ & $<1$ & 100 & 88 & -- & -- & -- & -- & -- & -- & 3.5 \\
\hline $04-30-85$ & -- & -- & -- & -- & -- & -- & -- & -- & -- & -- \\
\hline $05-07-85$ & -- & -- & -- & -- & -- & -- & -- & -- & -- & -- \\
\hline $05-28-85$ & -- & -- & -- & -- & -- & -- & -- & -- & -- & -- \\
\hline $06-25-85$ & -- & -- & -- & -- & -- & -- & -- & -- & -- & -- \\
\hline $07-23-85$ & -- & -- & -- & -- & -- & -- & -- & -- & -- & -- \\
\hline $08-07-85$ & -- & -- & -- & -- & -- & -- & -- & -- & -- & -- \\
\hline $08-26-85$ & -- & -- & 110 & -- & -- & -- & -- & -- & -- & 7.1 \\
\hline
\end{tabular}

1 Sample filtered through 0.1 micrometer pore filter. 
Table 5.--Physical characteristics and concentrations of chemical constituents of streamflow at station number 01174560, West Branch Swift River at Cooleyville, Massachusetts

$\left[\mathrm{ft}^{3} / \mathrm{s}\right.$, cubic feet per second; $\mu \mathrm{S} / \mathrm{cm}$, microsiemens per centimeter at 25 degrees Celsius; ${ }^{\circ} \mathrm{C}$, degrees Celsius; $\mathrm{mg} / \mathrm{L}$, milligrams per liter, $\mu \mathrm{g} / \mathrm{L}$, micrograms per liter; <, less than; dashes indicate not analyzed]

\begin{tabular}{|c|c|c|c|c|c|c|c|c|c|}
\hline Date & Time & $\begin{array}{l}\text { Stream- } \\
\text { flow, } \\
\text { instan- } \\
\text { taneous } \\
\left(\mathrm{ft}^{3} / \mathrm{s}\right)\end{array}$ & $\begin{array}{l}\text { Spe- } \\
\text { cific } \\
\text { con- } \\
\text { duct- } \\
\text { ance } \\
(\mu \mathrm{s} / \mathrm{cm})\end{array}$ & $\begin{array}{l}\mathrm{pH} \\
\text { (stand- } \\
\text { ard } \\
\text { units) }\end{array}$ & $\begin{array}{l}\text { Temper- } \\
\text { ature } \\
\left({ }^{\circ} \mathrm{C}\right)\end{array}$ & $\begin{array}{l}\text { Hard- } \\
\text { ness } \\
(\mathrm{mg} / \mathrm{L} \\
\mathrm{as} \\
\left.\mathrm{CaCO}_{3}\right)\end{array}$ & $\begin{array}{c}\text { Calcium, } \\
\text { dis- } \\
\text { solved } \\
\text { (mg/L } \\
\text { as Ca) }\end{array}$ & $\begin{array}{c}\text { Magne- } \\
\text { sium, } \\
\text { dis- } \\
\text { solved } \\
\text { (mg/L } \\
\text { as Mg) }\end{array}$ & $\begin{array}{c}\text { Sodium, } \\
\text { dis- } \\
\text { solved } \\
\text { (mg/I } \\
\text { as Na) }\end{array}$ \\
\hline $04-05-84$ & $09: 45$ & 87 & 29 & 5.2 & 1.5 & 5 & 1.6 & 0.32 & 2.1 \\
\hline $04-05-84^{1}$ & $09: 50$ & -- & -- & -- & -- & -- & -- & -- & -- \\
\hline $04-06-84$ & $11: 00$ & -- & 22 & 5.0 & -- & 4 & 1.3 & .23 & 1.5 \\
\hline $05-30-84$ & $13: 45$ & -- & 25 & 4.8 & 10.5 & 4 & 1.2 & .19 & 1.2 \\
\hline $05-30-84^{1}$ & $13: 50$ & -- & -- & -- & -- & -- & -- & -- & -- \\
\hline $08-28-84$ & $13: 40$ & -- & 28 & 6.3 & 16.5 & -- & -- & -- & -- \\
\hline $09-11-84$ & $15: 45$ & .16 & 29 & 6.3 & 16.0 & 6 & 1.8 & .34 & $2: 4$ \\
\hline $10-02-84$ & -- & -- & 29 & 5.9 & 8.5 & -- & -- & -- & -- \\
\hline $10-17-84$ & $08: 50$ & -- & 30 & 6.2 & 8.0 & -- & -- & -- & -- \\
\hline $12-19-84$ & $10: 30$ & 11 & 33 & 5.3 & 2.0 & 7 & 1.9 & .43 & 2.5 \\
\hline $04-30-85$ & $11: 45$ & -- & 30 & 5.9 & 15.0 & -- & -- & -- & -- \\
\hline $05-28-85$ & $11: 45$ & -- & 28 & 5.8 & 14.0 & -- & -- & -- & -- \\
\hline $06-25-85$ & $13: 45$ & -- & 27 & 6.2 & 13.5 & -- & -- & -- & -- \\
\hline $07-23-85$ & $12: 15$ & -- & 30 & 6.3 & 16.0 & -- & -- & -- & -- \\
\hline $08-07-85$ & $12: 30$ & -- & 32 & 5.9 & 15.5 & -- & -- & -- & -- \\
\hline $08-26-85$ & $12: 00$ & 13 & 30 & 5.3 & 16.0 & 6 & 1.9 & .31 & 2.0 \\
\hline
\end{tabular}

See footnote at end of table.

\begin{tabular}{|c|c|c|c|c|c|c|c|c|c|c|}
\hline Date & $\begin{array}{l}\text { Potas- } \\
\text { sium, } \\
\text { dis- } \\
\text { solved } \\
\text { (mg/L } \\
\text { as K) }\end{array}$ & $\begin{array}{l}\text { Nitro- } \\
\text { gen, } \\
\text { ammonia } \\
\text { total } \\
\text { (mg/L } \\
\text { as N) }\end{array}$ & $\begin{array}{l}\text { Nitro- } \\
\text { gen, } \\
\text { ammonia, } \\
\text { dis- } \\
\text { solved } \\
\text { (mg/L } \\
\text { as N) }\end{array}$ & $\begin{array}{c}\text { Alka- } \\
\text { linity } \\
\text { field } \\
\text { (mg/L } \\
\text { as } \\
\mathrm{CaCO}_{3} \text { ) }\end{array}$ & $\begin{array}{l}\text { Sulfate, } \\
\text { dis- } \\
\text { solved } \\
\text { (mg/L } \\
\text { as } \mathrm{SO}_{4} \text { ) }\end{array}$ & $\begin{array}{c}\text { Nitro- } \\
\text { gen, } \\
\text { nitrate, } \\
\text { dis- } \\
\text { solved } \\
\text { (mg/L } \\
\text { as N) }\end{array}$ & $\begin{array}{l}\text { Phos- } \\
\text { phorus, } \\
\text { ortho, } \\
\text { dis- } \\
\text { solved } \\
\text { (mg/L } \\
\text { as P) }\end{array}$ & $\begin{array}{l}\text { Chlo- } \\
\text { ride, } \\
\text { dis- } \\
\text { solved } \\
\text { (mg/L } \\
\text { as Cl) }\end{array}$ & $\begin{array}{l}\text { Flub- } \\
\text { ride, } \\
\text { dis- } \\
\text { solved } \\
\text { (mg/L } \\
\text { as F) }\end{array}$ & $\begin{array}{c}\text { Silica, } \\
\text { dis- } \\
\text { solved } \\
\text { (mg/L } \\
\text { as } \\
\mathrm{SiO}_{2} \text { ) }\end{array}$ \\
\hline $04-05-84$ & 0.30 & $<0.001$ & -- & 0.0 & 6.4 & -- & -- & 2.6 & $<0.01$ & 5.1 \\
\hline $04-05-84$ & -- & -- & -- & -- & -- & -- & -- & -- & -- & -- \\
\hline $04-06-84$ & .32 & -- & $<0.01$ & .3 & 6.1 & $<0.05$ & $<0.06$ & 1.8 & .05 & 4.0 \\
\hline $05-30-84$ & .29 & .030 & -- & .0 & 5.3 & -- & -- & 1.6 & .04 & 4.1 \\
\hline $05-30-84$ & -- & -- & -- & -- & -- & -- & -- & -- & -- & -- \\
\hline $08-28-84$ & -- & -- & -- & 1.4 & -- & -- & -- & -- & -- & -- \\
\hline $09-11-84$ & .51 & .064 & -- & 1.6 & 7.0 & -- & -- & 2.1 & .06 & 6.7 \\
\hline $10-02-84$ & -- & -- & -- & -- & -- & -- & -- & -- & -- & -- \\
\hline $10-17-84$ & -- & -- & -- & 1.8 & -- & -- & -- & -- & -- & -- \\
\hline $12-19-84$ & .32 & .023 & -- & .4 & 7.6 & .01 & $<.01$ & -- & .08 & 8.1 \\
\hline $04-30-85$ & -- & -- & -- & .7 & -- & -- & -- & -- & -- & -- \\
\hline $05-28-85$ & -- & -- & -- & .6 & -- & -- & -- & -- & -- & -- \\
\hline $06-25-85$ & -- & -- & -- & 1.0 & -- & -- & -- & -- & -- & -- \\
\hline $07-23-85$ & -- & -- & -- & -- & -- & -- & -- & -- & -- & -- \\
\hline $08-07-85$ & -- & -- & -- & 1.3 & -- & -- & -- & -- & -- & -- \\
\hline $08-26-85$ & .22 & -- & $<.01$ & .2 & 6.0 & .01 & $<.01$ & 1.7 & .1 & 6.9 \\
\hline
\end{tabular}


Table 5.--Physical characteristics and concentrations of chemical constituents of streamflow at station number 01174560, West Branch Swift River at Cooleyville, Massachusetts--Continued

\begin{tabular}{|c|c|c|c|c|c|c|c|c|c|c|}
\hline Date & $\begin{array}{l}\text { Solids, } \\
\text { sum of } \\
\text { constit- } \\
\text { uents, } \\
\text { dis- } \\
\text { solved } \\
\text { (mg/L) }\end{array}$ & $\begin{array}{l}\text { Alum- } \\
\text { inum, } \\
\text { total } \\
\text { recov- } \\
\text { erable } \\
(\mu \mathrm{g} / \mathrm{L} \\
\text { as Al) }\end{array}$ & $\begin{array}{l}\text { Alum- } \\
\text { inum, } \\
\text { dis- } \\
\text { solved } \\
(\mu g / L \\
\text { as Al) }\end{array}$ & $\begin{array}{c}\text { Arsenic, } \\
\text { dis- } \\
\text { solved } \\
(\mu g / L \\
\text { as As) }\end{array}$ & $\begin{array}{c}\text { Arsenic, } \\
\text { total } \\
\text { ( } \mu \text { g/L } \\
\text { as As) }\end{array}$ & $\begin{array}{c}\text { Copper, } \\
\text { total } \\
\text { recov- } \\
\text { erable } \\
(\mu g / L \\
\text { as } \mathrm{Cu})\end{array}$ & $\begin{array}{l}\text { Copper, } \\
\text { dis- } \\
\text { solved } \\
(\mu g / L \\
\text { as Cu) }\end{array}$ & $\begin{array}{l}\text { Iron, } \\
\text { total } \\
\text { recov- } \\
\text { erable } \\
(\mu g / L \\
\text { as } \mathrm{Fe})\end{array}$ & $\begin{array}{l}\text { Iron, } \\
\text { dis- } \\
\text { solved } \\
(\mu \mathrm{g} / \mathrm{L} \\
\text { as } \mathrm{Fe})\end{array}$ & $\begin{array}{l}\text { Lead, } \\
\text { total } \\
\text { recov- } \\
\text { erable } \\
(\mu g / L \\
\text { as } P b)\end{array}$ \\
\hline $04-05-84$ & -- & 240 & 180 & 1 & 1 & 1 & 2.0 & 130 & 27 & 1 \\
\hline $04-05-84$ & -- & -- & $1_{140}$ & -- & -- & -- & -- & -- & $1_{23}$ & -- \\
\hline $04-06-84$ & 16 & -- & -- & -- & -- & -- & -- & -- & 20 & -- \\
\hline $05-30-84$ & -- & -- & -- & -- & -- & -- & -- & -- & 70 & -- \\
\hline $05-30-84$ & -- & -- & $1_{280}$ & -- & -- & -- & -- & -- & ${ }^{1} 85$ & -- \\
\hline $08-28-84$ & -- & -- & -- & -- & -- & -- & -- & -- & -- & -- \\
\hline $09-11-84$ & -- & 20 & 10 & $<1$ & $<1$ & 1 & 1.1 & 100 & 14 & 2 \\
\hline $10-02-84$ & -- & -- & -- & -- & -- & -- & -- & - & -- & -- \\
\hline $10-17-84$ & -- & -- & -- & -- & -- & -- & -- & -- & -- & -- \\
\hline $12-19-84$ & -- & 160 & 160 & -- & -- & 1 & .1 & 160 & 44 & 3 \\
\hline $04-30-85$ & -- & -- & -- & -- & -- & -- & -- & -- & -- & -- \\
\hline $05-28-85$ & -- & -- & -- & -- & -- & -- & -- & -- & -- & -- \\
\hline $06-25-85$ & -- & -- & -- & -- & -- & -- & -- & -- & -- & -- \\
\hline $07-23-85$ & -- & -- & -- & -- & -- & -- & -- & -- & -- & -- \\
\hline $08-07-85$ & -- & -- & -- & -- & -- & -- & -- & -- & -- & -- \\
\hline $08-26-85$ & 20 & -- & 190 & -- & -- & -- & -- & -- & 82 & -- \\
\hline
\end{tabular}

See footnote at end of table.

\begin{tabular}{|c|c|c|c|c|c|c|c|c|c|c|}
\hline Date & $\begin{array}{l}\text { Lead, } \\
\text { dis- } \\
\text { solved } \\
(\mu g / L \\
\text { as } \mathrm{Pb})\end{array}$ & $\begin{array}{l}\text { Manga- } \\
\text { nese, } \\
\text { total } \\
\text { recov- } \\
\text { erable } \\
\text { ( } \mu \text { g } / \mathrm{L} \\
\text { as } \mathrm{Mn})\end{array}$ & $\begin{array}{l}\text { Manga- } \\
\text { nese, } \\
\text { dis- } \\
\text { solved } \\
\text { ( } \mu \text { g/L } \\
\text { as Mn) }\end{array}$ & $\begin{array}{l}\text { Mercury, } \\
\text { total } \\
\text { recov- } \\
\text { erable } \\
(\mu g / L \\
\text { as } \mathrm{Hg})\end{array}$ & $\begin{array}{c}\text { Mercury, } \\
\text { dis- } \\
\text { solved } \\
(\mu g / L \\
\text { as } H g)\end{array}$ & $\begin{array}{l}\text { Sele- } \\
\text { nium, } \\
\text { total } \\
\text { ( } \mu \mathrm{g} / \mathrm{L} \\
\text { as } \mathrm{Se})\end{array}$ & $\begin{array}{l}\text { Sele- } \\
\text { nium, } \\
\text { dis- } \\
\text { solved } \\
(\mu \mathrm{g} / \mathrm{L} \\
\text { as Se) }\end{array}$ & $\begin{array}{l}\text { Vana- } \\
\text { dium, } \\
\text { dis- } \\
\text { solved } \\
(\mu \mathrm{g} / \mathrm{L} \\
\text { as V) }\end{array}$ & $\begin{array}{l}\text { Carbon, } \\
\text { organic } \\
\text { total } \\
\text { (mg/L } \\
\text { as C) }\end{array}$ & $\begin{array}{c}\text { Carbon, } \\
\text { organic, } \\
\text { dis- } \\
\text { solved } \\
\text { (mg/L } \\
\text { as C) }\end{array}$ \\
\hline $04-05-84$ & 0.8 & 50 & 51 & $<0.1$ & $<0.1$ & $<1$ & $<1$ & $<1$ & 4.5 & -- \\
\hline $04-05-84$ & -- & -- & ${ }^{1} 48$ & -- & -- & -- & -- & - & -- & -- \\
\hline $04-06-84$ & -- & -- & 81 & -- & -- & -- & -- & -- & -- & -- \\
\hline $05-30-84$ & -- & -- & 70 & -- & -- & -- & -- & -- & 5.5 & -- \\
\hline $05-30-84$ & -- & -- & ${ }^{1} 75$ & -- & -- & -- & -- & -- & -- & -- \\
\hline $08-28-84$ & -- & -- & -- & -- & -- & -- & -- & -- & -- & -- \\
\hline $09-11-84$ & .5 & $<10$ & 4 & $<.1$ & $<.1$ & $<1$ & $<1$ & $<1$ & -- & 1.2 \\
\hline $10-02-84$ & -- & -- & -- & -- & -- & -- & - & -- & -- & -- \\
\hline $10-17-84$ & -- & -- & -- & -- & -- & -- & -- & -- & -- & -- \\
\hline $12-19-84$ & .7 & 60 & 53 & -- & -- & -- & -- & -- & -- & 3.1 \\
\hline $04-30-85$ & -- & -- & -- & -- & -- & -- & -- & -- & -- & -- \\
\hline $05-28-85$ & -- & -- & -- & -- & -- & -- & -- & -- & -- & -- \\
\hline $06-25-85$ & -- & -- & -- & -- & -- & -- & -- & -- & -- & -- \\
\hline $07-23-85$ & -- & -- & -- & -- & -- & -- & -- & -- & -- & -- \\
\hline $08-07-85$ & -- & -- & -- & -- & -- & -- & -- & -- & -- & -- \\
\hline $08-26-85$ & -- & -- & 43 & -- & -- & -- & -- & -- & -- & 5.7 \\
\hline
\end{tabular}

1 Sample filtered through 0.1 micrometer pore filter. 
Table 6.--Physical characteristics and concentrations of chemical constituents of streamflow at station number 01174563, West Branch Swift River tributary at Cooleyville, Massachusetts

$\left[\mathrm{ft}^{3} / \mathrm{s}\right.$, cubic feet per second; $\mu \mathrm{S} / \mathrm{cm}$, microsiemens per centimeter at 25 degrees Celsius; ${ }^{\circ} \mathrm{C}$, degrees Celsius; $\mathrm{mg} / \mathrm{L}$, milligrams per liter; $\mu \mathrm{g} / \mathrm{L}$, micrograms per liter, <, less than; dashes indicate not analyzed]

\begin{tabular}{|c|c|c|c|c|c|c|c|c|c|}
\hline Date & Time & $\begin{array}{l}\text { Stream- } \\
\text { flow, } \\
\text { instan- } \\
\text { taneous } \\
\left(\mathrm{ft}^{3} / \mathrm{s}\right)\end{array}$ & $\begin{array}{l}\text { Spe- } \\
\text { cific } \\
\text { con- } \\
\text { duct- } \\
\text { ance } \\
(\mu \mathrm{s} / \mathrm{cm})\end{array}$ & $\begin{array}{c}\mathrm{pH} \\
\text { (stand- } \\
\text { ard } \\
\text { units) }\end{array}$ & $\begin{array}{l}\text { Temper- } \\
\text { ature } \\
\left({ }^{\circ} \mathrm{C}\right)\end{array}$ & $\begin{array}{l}\text { Hard- } \\
\text { ness } \\
(\mathrm{mg} / \mathrm{L} \\
\mathrm{as} \\
\left.\mathrm{CaCO}_{3}\right)\end{array}$ & $\begin{array}{c}\text { Calcium, } \\
\text { dis- } \\
\text { solved } \\
\text { (mg/L } \\
\text { as Ca) }\end{array}$ & $\begin{array}{l}\text { Magne- } \\
\text { sium, } \\
\text { dis- } \\
\text { solved } \\
\text { (mg/L } \\
\text { as Mg) }\end{array}$ & $\begin{array}{l}\text { Sodium, } \\
\text { dis- } \\
\text { solved } \\
\text { (mg/L } \\
\text { as Na) }\end{array}$ \\
\hline $04-05-84$ & $09: 30$ & 44 & 21 & 5.8 & 2.0 & 5 & 1.5 & 0.33 & 1.2 \\
\hline $04-05-84^{1}$ & $09: 45$ & -- & -- & -- & -- & 5 & 1.5 & .34 & 1.2 \\
\hline $04-06-84$ & $11: 00$ & - & 20 & 5.2 & -- & 4 & 1.2 & .26 & .9 \\
\hline $05-30-84$ & $13: 30$ & -- & 21 & 4.9 & 10.5 & 4 & 1.2 & .23 & .8 \\
\hline $05-30-84^{1}$ & $13: 40$ & -- & -- & -- & -- & -- & -- & -- & -- \\
\hline $08-28-84$ & $13: 30$ & -- & 20 & 6.0 & 20.0 & -- & -- & -- & -- \\
\hline $09-11-84$ & $15: 00$ & .2 & 21 & 6.1 & 21.5 & 5 & 1.4 & .29 & 1.6 \\
\hline $10-02-84$ & -- & -- & 28 & 5.9 & 9.0 & -- & -- & -- & -- \\
\hline $10-17-84$ & $08: 30$ & -- & 21 & 6.0 & 8.0 & -- & -- & -- & -- \\
\hline $12-19-84$ & $08: 30$ & 4.4 & 28 & 5.9 & 2.0 & 6 & 1.9 & .41 & 1.8 \\
\hline $04-30-85$ & $11: 30$ & -- & 26 & 6.0 & 11.5 & -- & -- & -- & -- \\
\hline $05-28-85$ & $11: 30$ & -- & 24 & 5.9 & 16.0 & -- & -- & -- & -- \\
\hline $06-25-85$ & $14: 00$ & -- & 23 & 5.8 & 17.5 & -- & -- & -- & -- \\
\hline $07-23-85$ & $12: 00$ & -- & 22 & 6.0 & 21.5 & -- & -- & -- & -- \\
\hline $08-07-85$ & $12: 45$ & -- & 21 & 5.8 & 20.0 & -- & -- & -- & -- \\
\hline $08-26-85$ & $13: 15$ & 5.4 & 25 & 5.6 & 17.0 & 6 & 1.9 & .38 & 1.5 \\
\hline
\end{tabular}

see footnote at end of table.

\begin{tabular}{|c|c|c|c|c|c|c|c|c|c|c|}
\hline Date & $\begin{array}{l}\text { Potas- } \\
\text { sium, } \\
\text { dis- } \\
\text { solved } \\
\text { (mg/L } \\
\text { as K) }\end{array}$ & $\begin{array}{l}\text { Nitro- } \\
\text { gen, } \\
\text { ammonia } \\
\text { total } \\
\text { (mg/L } \\
\text { as N) }\end{array}$ & $\begin{array}{l}\text { Nitro- } \\
\text { gen, } \\
\text { ammonia, } \\
\text { dis- } \\
\text { solved } \\
\text { (mg/L } \\
\text { as N) }\end{array}$ & $\begin{array}{c}\text { Alka- } \\
\text { linity } \\
\text { field } \\
\text { (mg/L } \\
\text { as } \\
\mathrm{CaCO}_{3} \text { ) }\end{array}$ & $\begin{array}{l}\text { Sulfate, } \\
\text { dis- } \\
\text { solved } \\
\text { (mg/L } \\
\text { as } \mathrm{SO}_{4} \text { ) }\end{array}$ & $\begin{array}{c}\text { Nitro- } \\
\text { gen, } \\
\text { nitrate, } \\
\text { dis- } \\
\text { solved } \\
\text { (mg/L } \\
\text { as N) }\end{array}$ & $\begin{array}{l}\text { Phos- } \\
\text { phorus, } \\
\text { ortho, } \\
\text { dis- } \\
\text { solved } \\
\text { (mg/L } \\
\text { as P) }\end{array}$ & $\begin{array}{l}\text { Chlo- } \\
\text { ride, } \\
\text { dis- } \\
\text { solved } \\
\text { (mg/L } \\
\text { as Cl) }\end{array}$ & $\begin{array}{l}\text { Fluo- } \\
\text { ride, } \\
\text { dis- } \\
\text { solved } \\
\text { (mg/L } \\
\text { as F) }\end{array}$ & $\begin{array}{l}\text { silica, } \\
\text { dis- } \\
\text { solved } \\
\text { (mg/L } \\
\text { as } \\
\mathrm{SiO}_{2} \text { ) }\end{array}$ \\
\hline $04-05-84$ & 0.22 & $<0.001$ & -- & 0.2 & 5.9 & -- & -- & 0.78 & $<0.01$ & 5.5 \\
\hline $04-05-84$ & -- & -- & -- & -- & -- & -- & -- & -- & -- & 5.5 \\
\hline $04-06-84$ & .25 & -- & $<0.01$ & .3 & 6.0 & $<0.05$ & $<0.06$ & .78 & .05 & 4.4 \\
\hline $05-30-84$ & .24 & .066 & -- & .0 & 5.0 & -- & -- & .69 & .04 & 4.5 \\
\hline $05-30-84$ & -- & -- & -- & -- & -- & -- & -- & -- & -- & -- \\
\hline $08-28-84$ & -- & -- & -- & 1.3 & -- & -- & -- & -- & -- & -- \\
\hline $09-11-84$ & .35 & .081 & -- & 1.2 & 5.2 & - & -- & 1.0 & .08 & 5.2 \\
\hline $10-02-84$ & -- & -- & -- & -- & -- & -- & -- & $-\infty$ & -- & -- \\
\hline $10-17-84$ & -- & -- & -- & 1.4 & -- & -- & -- & -- & -- & -- \\
\hline $12-19-84$ & .33 & .015 & -- & .4 & -- & .01 & -- & 1.2 & .06 & 7.8 \\
\hline $04-30-85$ & -- & -- & -- & 1.0 & -- & -- & -- & - & -- & -- \\
\hline $05-28-85$ & -- & -- & -- & 1.2 & -- & -- & -- & -- & -- & -- \\
\hline $06-25-85$ & -- & -- & -- & 1.9 & -- & -- & -- & -- & -- & -- \\
\hline $07-23-85$ & -- & -- & -- & -- & -- & -- & -- & -- & -- & -- \\
\hline $08-07-85$ & -- & -- & -- & .7 & -- & $--\quad$ & -- & -- & -- & -- \\
\hline $08-26-85$ & .22 & -- & $<.01$ & .7 & 5.7 & .01 & $<.01$ & 1.0 & .06 & 6.4 \\
\hline
\end{tabular}


Table 6.--Physical characteristics and concentrations of chemical constituents of streamflow at station number 01174563, West Branch Swift River tributary at Cooleyville, Massachusetts--Continued

\begin{tabular}{|c|c|c|c|c|c|c|c|c|c|c|}
\hline Date & $\begin{array}{l}\text { Solids, } \\
\text { sum of } \\
\text { consti- } \\
\text { tuents, } \\
\text { dis- } \\
\text { solved } \\
\text { (mg/L) }\end{array}$ & $\begin{array}{l}\text { Alum- } \\
\text { inum, } \\
\text { total } \\
\text { recov- } \\
\text { erable } \\
(\mu \mathrm{g} / \mathrm{L} \\
\text { as } \mathrm{Al})\end{array}$ & $\begin{array}{l}\text { Alum- } \\
\text { inum, } \\
\text { dis- } \\
\text { solved } \\
(\mu \mathrm{g} / \mathrm{L} \\
\text { as Al) }\end{array}$ & $\begin{array}{c}\text { Arsenic, } \\
\text { dis- } \\
\text { solved } \\
\text { ( } \mu \text { g/L } \\
\text { as As) }\end{array}$ & $\begin{array}{c}\text { Arsenic, } \\
\text { total } \\
\text { ( } \mu \text { g/L } \\
\text { as As) }\end{array}$ & $\begin{array}{c}\text { Copper, } \\
\text { total } \\
\text { recov- } \\
\text { erable } \\
(\mu g / L \\
\text { as } C u)\end{array}$ & $\begin{array}{l}\text { Copper, } \\
\text { dis- } \\
\text { solved } \\
(\mu g / L \\
\text { as } \mathrm{Cu})\end{array}$ & $\begin{array}{l}\text { Iron, } \\
\text { total } \\
\text { recov- } \\
\text { erable } \\
(\mu \mathrm{g} / \mathrm{L} \\
\text { as Fe) }\end{array}$ & $\begin{array}{l}\text { Iron, } \\
\text { dis- } \\
\text { solved } \\
(\mu \mathrm{g} / \mathrm{L} \\
\text { as } \mathrm{Fe})\end{array}$ & $\begin{array}{c}\text { Lead, } \\
\text { total } \\
\text { recov- } \\
\text { erable } \\
(\mu g / L \\
\text { as } \mathrm{Pb})\end{array}$ \\
\hline $04-05-84$ & 16 & 320 & 150 & $<1$ & 1 & 1 & 1.2 & 160 & 30 & 1 \\
\hline $04-05-84$ & -- & -- & ${ }^{1} 110$ & -- & -- & -- & -- & -- & $1_{25}$ & -- \\
\hline $04-06-84$ & 14 & -- & -- & -- & -- & -- & -- & & 16 & - \\
\hline $05-30-84$ & -- & -- & -- & -- & -- & -- & -- & -- & 69 & -- \\
\hline $05-30-84$ & -- & -- & ${ }^{1} 260$ & -- & -- & -- & -- & -- & ${ }^{1} 65$ & -- \\
\hline $08-28-84$ & -- & -- & -- & -- & -- & -- & -- & -- & -- & -- \\
\hline $09-11-84$ & -- & 80 & 50 & $<1$ & $<1$ & 1 & 2.3 & 290 & 88 & 2 \\
\hline $10-02-84$ & -- & - & -- & -- & -- & -- & -- & -- & -- & -- \\
\hline $10-17-84$ & -- & -- & -- & -- & -- & -- & -- & -- & -- & -- \\
\hline $12-19-84$ & -- & $<10$ & $<10$ & -- & -- & 1 & 1.6 & 130 & 38 & 6 \\
\hline $04-30-85$ & -- & -- & -- & -- & -- & -- & -- & -- & -- & -- \\
\hline $05-28-85$ & -- & -- & -- & -- & -- & -- & -- & -- & -- & -- \\
\hline $06-25-85$ & - & -- & -- & -- & -- & -- & -- & -- & -- & -- \\
\hline $07-23-85$ & -- & - & -- & -- & -- & -- & - & -- & - & -- \\
\hline $08-07-85$ & -- & -- & -- & -- & -- & -- & -- & -- & -- & -- \\
\hline $08-26-85$ & 18 & -- & 160 & -- & -- & -- & -- & -- & 130 & -- \\
\hline
\end{tabular}

See footnote at end of table.

\begin{tabular}{|c|c|c|c|c|c|c|c|c|c|c|}
\hline Date & $\begin{array}{l}\text { Lead, } \\
\text { dis- } \\
\text { solved } \\
(\mu g / L \\
\text { as } \mathrm{Pb})\end{array}$ & $\begin{array}{l}\text { Manga- } \\
\text { nese, } \\
\text { total } \\
\text { recov- } \\
\text { erable } \\
\text { ( } \mu \text { g/L } \\
\text { as } M n)\end{array}$ & $\begin{array}{l}\text { Manga- } \\
\text { nese, } \\
\text { dis- } \\
\text { solved } \\
(\mu g / L \\
\text { as Mn) }\end{array}$ & $\begin{array}{c}\text { Mercury, } \\
\text { total } \\
\text { recov- } \\
\text { erable } \\
(\mu g / L \\
\text { as } \mathrm{Hg})\end{array}$ & $\begin{array}{c}\text { Mercury, } \\
\text { dis- } \\
\text { solved } \\
\text { ( } \mu g / L \\
\text { as } \mathrm{Hg})\end{array}$ & $\begin{array}{l}\text { Sele- } \\
\text { nium, } \\
\text { total } \\
(\mu g / L \\
\text { as } \mathrm{Se})\end{array}$ & $\begin{array}{l}\text { Sele- } \\
\text { nium, } \\
\text { dis- } \\
\text { solved } \\
\text { ( } \mu \text { g/L } \\
\text { as } S e)\end{array}$ & $\begin{array}{l}\text { Vana- } \\
\text { dium, } \\
\text { dis- } \\
\text { solved } \\
(\mu g / L \\
\text { as V) }\end{array}$ & $\begin{array}{c}\text { Carbon, } \\
\text { organic } \\
\text { total } \\
\text { (mg/L } \\
\text { as C) }\end{array}$ & $\begin{array}{c}\text { Carbon, } \\
\text { organic, } \\
\text { dis- } \\
\text { solved } \\
\text { (mg/L } \\
\text { as C) }\end{array}$ \\
\hline $04-05-84$ & 0.5 & 50 & 38 & $<0.1$ & $<0.1$ & $<1$ & $<1$ & $<1$ & 2.7 & -- \\
\hline $04-05-84$ & - & - & $1_{31}$ & -- & -- & -- & - & - & - & -- \\
\hline $04-06-84$ & -- & -- & 56 & -- & -- & -- & -- & -- & -- & -- \\
\hline $05-30-84$ & -- & -- & 53 & -- & -- & -- & -- & -- & 4.8 & -- \\
\hline $05-30-84$ & -- & -- & ${ }^{1} 53$ & -- & -- & -- & -- & -- & -- & -- \\
\hline $08-28-84$ & -- & -- & -- & -- & -- & - & -- & -- & -- & -- \\
\hline $09-11-84$ & .4 & 20 & 16 & .6 & $<.1$ & $<1$ & $<1$ & $<1$ & -- & 2.3 \\
\hline $10-02-84$ & -- & -- & -- & -- & -- & -- & -- & -- & -- & - \\
\hline $10-17-84$ & -- & -- & -- & -- & -- & -- & -- & -- & - & -- \\
\hline $12-19-84$ & 1.1 & $<10$ & 5 & -- & -- & -- & -- & -- & -- & 2.8 \\
\hline $04-30-85$ & -- & -- & -- & -- & -- & - & -- & -- & -- & -- \\
\hline $05-28-85$ & -- & -- & - & -- & -- & -- & -- & -- & - & -- \\
\hline $06-25-85$ & -- & -- & -- & -- & -- & -- & -- & -- & - & -- \\
\hline $07-23-85$ & -- & -- & -- & -- & -- & -- & -- & -- & -- & -- \\
\hline $08-07-85$ & -- & -- & -- & -- & -- & -- & -- & -- & -- & -- \\
\hline $08-26-85$ & -- & -- & 24 & -- & -- & -- & -- & -- & -- & 6.5 \\
\hline
\end{tabular}

1 Sample filtered through 0.1 micrometer pore filter. 
Table 7.--Physical characteristics and concentrations of chemical constituents of streamflow at station number 01174564, West Branch Swift River tributary 2 at Cooleyville, Massachusetts

$\left[\mathrm{ft}^{3} / \mathrm{s}\right.$, cubic feet per second; $\mu \mathrm{S} / \mathrm{cm}$, microsiemens per centimeter at 25 degrees Celsius; ${ }^{\circ} \mathrm{C}$, degrees Celsius; $\mathrm{mg} / \mathrm{L}$, milligrams per liter; $\mu \mathrm{g} / \mathrm{L}$, micrograms per liter, <, less than; dashes indicate not analyzed]

\begin{tabular}{|c|c|c|c|c|c|c|c|c|c|}
\hline Date & Time & $\begin{array}{l}\text { Stream- } \\
\text { flow, } \\
\text { instan- } \\
\text { taneous } \\
\left(\mathrm{ft}^{3} / \mathrm{s}\right)\end{array}$ & $\begin{array}{l}\text { Spe- } \\
\text { cific } \\
\text { con- } \\
\text { duct- } \\
\text { ance } \\
(\mu s / \mathrm{cm})\end{array}$ & $\begin{array}{c}\mathrm{pH} \\
\text { (stand- } \\
\text { ard } \\
\text { units) }\end{array}$ & $\begin{array}{l}\text { Temper- } \\
\text { ature } \\
\left({ }^{\circ} \mathrm{C}\right)\end{array}$ & $\begin{array}{c}\text { Hard- } \\
\text { ness } \\
(\mathrm{mg} / \mathrm{L} \\
\mathrm{as} \\
\mathrm{CaCO}_{3} \text { ) }\end{array}$ & $\begin{array}{l}\text { Calcium, } \\
\text { dis- } \\
\text { solved } \\
\text { (mg/L } \\
\text { as Ca) }\end{array}$ & $\begin{array}{l}\text { Magne- } \\
\text { sium, } \\
\text { dis- } \\
\text { solved } \\
\text { (mg/L } \\
\text { as Mg) }\end{array}$ & $\begin{array}{l}\text { Sodium, } \\
\text { dis- } \\
\text { solved } \\
\text { (mg/L } \\
\text { as } \mathrm{Na} \text { ) }\end{array}$ \\
\hline $04-05-84$ & $08: 00$ & 14 & 71 & 6.6 & 4.0 & 12 & 3.5 & 0.69 & 7.8 \\
\hline $04-05-84^{1}$ & $08: 10$ & -- & - & -- & -- & - & -- & -- & - \\
\hline $04-06-84$ & $11: 00$ & -- & 55 & 6.6 & -- & 10 & 2.9 & .62 & 6.2 \\
\hline $05-30-84$ & $13: 25$ & -- & 39 & 6.5 & 11.0 & 8 & 2.4 & .40 & 4.3 \\
\hline $05-30-84^{1}$ & $13: 30$ & -- & -- & -- & -- & -- & -- & -- & -- \\
\hline $08-28-84$ & $12: 20$ & -- & 79 & 7.3 & 17.0 & -- & -- & -- & -- \\
\hline $09-11-84$ & $14: 30$ & .24 & 77 & 7.2 & 17.5 & 19 & 5.6 & 1.2 & 6.3 \\
\hline $10-02-84$ & -- & -- & 90 & 7.0 & 9.0 & -- & -- & -- & -- \\
\hline $10-17-84$ & $08: 15$ & -- & 86 & 7.1 & 9.0 & -- & -- & -- & -- \\
\hline $12-18-84$ & $14: 45$ & .84 & 105 & 7.0 & 4.0 & 19 & 5.6 & 1.2 & 9.6 \\
\hline $04-30-85$ & $11: 15$ & -- & 86 & 6.9 & 11.0 & -- & -- & -- & -- \\
\hline $05-28-85$ & $11: 00$ & -- & 99 & 6.9 & 13.5 & -- & -- & -- & -- \\
\hline $06-25-85$ & $14: 15$ & -- & 89 & 7.0 & 14.5 & -- & -- & -- & -- \\
\hline $07-23-85$ & $11: 55$ & -- & 93 & 7.1 & 17.0 & -- & -- & -- & -- \\
\hline $08-07-85$ & $13: 00$ & -- & 105 & 7.1 & 17.5 & -- & -- & -- & -- \\
\hline $08-26-85$ & $14: 30$ & .68 & 103 & 7.1 & 16.0 & 20 & 6.0 & 1.3 & 8.2 \\
\hline
\end{tabular}

See footnote at end of table.

\begin{tabular}{|c|c|c|c|c|c|c|c|c|c|c|}
\hline Date & $\begin{array}{l}\text { Potas- } \\
\text { sium, } \\
\text { dis- } \\
\text { solved } \\
\text { (mg/L } \\
\text { as K) }\end{array}$ & $\begin{array}{l}\text { Nitro- } \\
\text { gen, } \\
\text { ammonia } \\
\text { total } \\
\text { (mg/L } \\
\text { as N) }\end{array}$ & $\begin{array}{l}\text { Nitro- } \\
\text { gen, } \\
\text { ammonia, } \\
\text { dis- } \\
\text { solved } \\
\text { (mg/L } \\
\text { as } N \text { ) }\end{array}$ & $\begin{array}{c}\text { Alka- } \\
\text { linity } \\
\text { field } \\
\text { (mg/L } \\
\text { as } \\
\mathrm{CaCO}_{3} \text { ) }\end{array}$ & $\begin{array}{l}\text { Sulfate, } \\
\text { dis- } \\
\text { solved } \\
\text { (mg/L } \\
\text { as } \mathrm{SO}_{4} \text { ) }\end{array}$ & $\begin{array}{c}\text { Nitro- } \\
\text { gen, } \\
\text { nitrate, } \\
\text { dis- } \\
\text { solved } \\
\text { (mg/L } \\
\text { as } N \text { ) }\end{array}$ & $\begin{array}{l}\text { Phos- } \\
\text { phorus, } \\
\text { ortho, } \\
\text { dis- } \\
\text { solved } \\
\text { (mg/L } \\
\text { as P) }\end{array}$ & $\begin{array}{l}\text { Chlo- } \\
\text { ride, } \\
\text { dis- } \\
\text { solved } \\
\text { (mg/L } \\
\text { as Cl) }\end{array}$ & $\begin{array}{l}\text { Fluo- } \\
\text { ride, } \\
\text { dis- } \\
\text { solved } \\
\text { (mg/L } \\
\text { as F) }\end{array}$ & $\begin{array}{l}\text { Silica, } \\
\text { dis- } \\
\text { solved } \\
\text { (mg/L } \\
\text { as } \\
\mathrm{SiO}_{2} \text { ) }\end{array}$ \\
\hline $04-05-84$ & 0.61 & 0.036 & -- & 4.9 & 5.7 & -- & -- & 12 & $<0.01$ & 7.8 \\
\hline $04-05-84$ & -- & -- & -- & -- & -- & -- & -- & -- & -- & -- \\
\hline $04-06-84$ & .54 & -- & 0.05 & 4.1 & 7.7 & $<0.05$ & $<0.06$ & 9.0 & .08 & 7.9 \\
\hline $05-30-84$ & .50 & .043 & -- & -- & 6.2 & -- & -- & 4.9 & .04 & 6.3 \\
\hline $05-30-84$ & -- & -- & -- & -- & -- & -- & -- & -- & -- & -- \\
\hline $08-28-84$ & -- & -- & -- & 15.0 & -- & -- & -- & -- & -- & -- \\
\hline $09-11-84$ & 1.0 & .225 & -- & 14.3 & 7.9 & -- & -- & 8.7 & .11 & 11 \\
\hline $10-02-84$ & -- & -- & -- & -- & -- & -- & -- & -- & -- & -- \\
\hline $10-17-84$ & -- & -- & -- & -- & -- & -- & -- & -- & -- & -- \\
\hline $12-18-84$ & 1.0 & .004 & -- & 9.4 & 8.8 & -- & $<.01$ & 23 & -- & 11 \\
\hline $04-30-85$ & -- & -- & -- & 9.0 & -- & -- & -- & -- & -- & -- \\
\hline $05-28-85$ & -- & -- & -- & 9.8 & -- & -- & -- & -- & -- & -- \\
\hline $06-25-85$ & -- & -- & -- & 11.5 & -- & -- & -- & -- & -- & -- \\
\hline $07-23-85$ & -- & -- & -- & -- & -- & -- & -- & -- & -- & -- \\
\hline $08-07-85$ & -- & -- & -- & 13.1 & -- & -- & -- & -- & -- & -- \\
\hline $08-26-85$ & 1.0 & -- & $<.01$ & 11.5 & 7.7 & .06 & $<.01$ & 13 & .11 & 11 \\
\hline
\end{tabular}


Table 7.--Physical characteristics and concentrations of chemical constituents of streamflow at station number 01174564, West Branch Swift River tributary 2 at Cooleyville, Massachusetts--Continued

\begin{tabular}{|c|c|c|c|c|c|c|c|c|c|c|}
\hline Date & $\begin{array}{l}\text { Solids, } \\
\text { sum of } \\
\text { consti- } \\
\text { tuents, } \\
\text { dis- } \\
\text { solved } \\
\text { (mg/L) }\end{array}$ & $\begin{array}{l}\text { Alum- } \\
\text { inum, } \\
\text { total } \\
\text { recov- } \\
\text { erable } \\
\text { ( } \mu \text { g } / L \\
\text { as Al) }\end{array}$ & $\begin{array}{l}\text { Alum- } \\
\text { inum, } \\
\text { dis- } \\
\text { solved } \\
(\mu \mathrm{g} / \mathrm{L} \\
\text { as Al) }\end{array}$ & $\begin{array}{l}\text { Arsenic, } \\
\text { dis- } \\
\text { solved } \\
\text { ( } \mu \text { g/L } \\
\text { as As) }\end{array}$ & $\begin{array}{c}\text { Arsenic, } \\
\text { total } \\
\text { ( } \mu g / L \\
\text { as As) }\end{array}$ & $\begin{array}{l}\text { Copper, } \\
\text { total } \\
\text { recov- } \\
\text { erable } \\
(\mu g / L \\
\text { as } C u)\end{array}$ & $\begin{array}{l}\text { Copper, } \\
\text { dis- } \\
\text { solved } \\
(\mu g / L \\
\text { as Cu) }\end{array}$ & $\begin{array}{l}\text { Iron, } \\
\text { total } \\
\text { recov- } \\
\text { erable } \\
(\mu g / L \\
\text { as } \mathrm{Fe})\end{array}$ & $\begin{array}{c}\text { Iron, } \\
\text { dis- } \\
\text { solved } \\
(\mu g / L \\
\text { as } \mathrm{Fe})\end{array}$ & $\begin{array}{r}\text { Lead, } \\
\text { total } \\
\text { recov- } \\
\text { erable } \\
(\mu g / L \\
\text { as } \mathrm{Pb})\end{array}$ \\
\hline $04-05-84$ & 41 & 600 & 70 & $<1$ & 1 & 2 & 0.2 & 650 & 51 & 2 \\
\hline $04-05-84$ & -- & - & $1<10$ & -- & -- & -- & -- & -- & ${ }^{1} 15$ & -- \\
\hline $04-06-84$ & 38 & -- & -- & -- & -- & -- & -- & -- & 7 & -- \\
\hline $05-30-84$ & -- & -- & -- & -- & -- & -- & -- & -- & 43 & -- \\
\hline $05-30-84$ & -- & -- & ${ }^{1} 110$ & -- & -- & -- & -- & -- & ${ }^{1} 35$ & -- \\
\hline $08-28-84$ & -- & -- & -- & -- & -- & -- & -- & -- & -- & -- \\
\hline $09-11-84$ & -- & 20 & 10 & $<1$ & $<1$ & 1 & .4 & 280 & 200 & 3 \\
\hline $10-02-84$ & -- & -- & -- & -- & -- & -- & -- & -- & -- & -- \\
\hline $10-17-84$ & -- & -- & -- & -- & -- & -- & -- & -- & -- & -- \\
\hline $12-18-84$ & 66 & $<10$ & $<10$ & -- & -- & $<1$ & .3 & 250 & 73 & 2 \\
\hline $04-30-85$ & -- & -- & -- & -- & -- & -- & -- & -- & -- & -- \\
\hline $05-28-85$ & -- & -- & -- & -- & -- & -- & -- & -- & -- & -- \\
\hline $06-25-85$ & -- & -- & -- & -- & -- & -- & -- & -- & -- & -- \\
\hline $07-23-85$ & -- & -- & -- & -- & -- & -- & -- & -- & -- & -- \\
\hline $08-07-85$ & -- & -- & -- & -- & -- & -- & -- & -- & -- & -- \\
\hline $08-26-85$ & 56 & -- & 60 & -- & -- & -- & -- & -- & 200 & -- \\
\hline
\end{tabular}

See footnote at end of table.

\begin{tabular}{|c|c|c|c|c|c|c|c|c|c|c|}
\hline Date & $\begin{array}{l}\text { Lead, } \\
\text { dis- } \\
\text { solved } \\
\text { ( } \mu \mathrm{g} / \mathrm{L} \\
\text { as } \mathrm{Pb} \text { ) }\end{array}$ & $\begin{array}{l}\text { Manga- } \\
\text { nese, } \\
\text { total } \\
\text { recov- } \\
\text { erable } \\
(\mu g / L \\
\text { as } M n)\end{array}$ & $\begin{array}{l}\text { Manga- } \\
\text { nese, } \\
\text { dis- } \\
\text { solved } \\
\text { ( } \mu \mathrm{g} / \mathrm{L} \\
\text { as } \mathrm{Mn})\end{array}$ & $\begin{array}{l}\text { Mercury, } \\
\text { total } \\
\text { recov- } \\
\text { erable } \\
(\mu g / L \\
\text { as Hg) }\end{array}$ & $\begin{array}{c}\text { Mercury, } \\
\text { dis- } \\
\text { solved } \\
(\mu g / L \\
\text { as Hg) }\end{array}$ & $\begin{array}{l}\text { Sele- } \\
\text { nium, } \\
\text { total } \\
\text { ( } \mu \text { g/L } \\
\text { as Se) }\end{array}$ & $\begin{array}{l}\text { Sele- } \\
\text { nium, } \\
\text { dis- } \\
\text { solved } \\
\text { ( } \mu \text { g/L } \\
\text { as Se) }\end{array}$ & $\begin{array}{l}\text { Vana- } \\
\text { dium, } \\
\text { dis- } \\
\text { solved } \\
(\mu g / L \\
\text { as V) }\end{array}$ & $\begin{array}{c}\text { Carbon, } \\
\text { organic } \\
\text { total } \\
\text { (mg/L } \\
\text { as C) }\end{array}$ & $\begin{array}{l}\text { Carbon, } \\
\text { organic, } \\
\text { dis- } \\
\text { solved } \\
\text { (mg/L } \\
\text { as C) }\end{array}$ \\
\hline $04-05-84$ & 2.5 & 30 & 6 & $<0.1$ & 0.1 & $<1$ & $<1$ & $<1$ & 3.8 & -- \\
\hline $04-05-84$ & - & -- & $1<1$ & -- & -- & -- & - & - & -- & -- \\
\hline $04-06-84$ & -- & -- & 1 & -- & -- & -- & -- & -- & -- & -- \\
\hline $05-30-84$ & - & -- & 2 & -- & -- & -- & -- & - & 3.7 & -- \\
\hline $05-30-84$ & -- & -- & $1_{2}$ & -- & -- & -- & -- & -- & -- & -- \\
\hline $08-28-84$ & -- & -- & -- & -- & -- & -- & -- & -- & -- & -- \\
\hline $09-11-84$ & .3 & $<10$ & 2 & $<.1$ & $<.1$ & $<1$ & $<1$ & $<1$ & -- & 1.1 \\
\hline $10-02-84$ & -- & -- & -- & -- & -- & - & -- & -- & -- & -- \\
\hline $10-17-84$ & -- & -- & -- & -- & -- & -- & -- & -- & -- & -- \\
\hline $12-18-84$ & 1.0 & 10 & 4 & -- & -- & -- & -- & -- & -- & 1.8 \\
\hline $04-30-85$ & - & -- & -- & - & -- & $\ldots$ & -- & -- & -- & -- \\
\hline $05-28-85$ & -- & -- & -- & - & -- & -- & -- & -- & -- & -- \\
\hline $06-25-85$ & -- & -- & -- & -- & -- & -- & -- & -- & -- & -- \\
\hline $07-23-85$ & -- & -- & -- & -- & -- & -- & -- & -- & -- & -- \\
\hline $08-07-85$ & -- & -- & -- & -- & -- & -- & -- & -- & - & -- \\
\hline $08-26-85$ & -- & -- & 5 & -- & -- & -- & -- & -- & -- & 3.7 \\
\hline
\end{tabular}

1 Sample filtered through 0.1 micrometer pore filter. 
Table 8.--Physical characteristics and concentrations of chemical constituents of ground water at station number 422800072232901, Swift River till well near Cooleyville, Massachusetts

$\left[\mu \mathrm{S} / \mathrm{cm}\right.$, microsiemens per centimeter at 25 degrees Celsius; ${ }^{\circ} \mathrm{C}$, degrees Celsius; $\mathrm{mg} / \mathrm{L}$, milligrams per liter; $\mu \mathrm{g} / \mathrm{L}$, micrograms per liter, <, less than; dashes indicate not analyzed]

\begin{tabular}{|c|c|c|c|c|c|c|c|c|}
\hline Date & Time & $\begin{array}{l}\text { Spe- } \\
\text { cific } \\
\text { con- } \\
\text { duct- } \\
\text { ance } \\
(\mu s / \mathrm{cm})\end{array}$ & $\begin{array}{c}\mathrm{pH} \\
\text { (stand- } \\
\text { ard } \\
\text { units) }\end{array}$ & $\begin{array}{l}\text { Temper- } \\
\text { ature } \\
\left({ }^{\circ} \mathrm{C}\right)\end{array}$ & $\begin{array}{l}\text { Hard- } \\
\text { ness } \\
(\mathrm{mg} / \mathrm{L} \\
\mathrm{ass} \\
\left.\mathrm{CaCO}_{3}\right)\end{array}$ & $\begin{array}{l}\text { Calcium, } \\
\text { dis- } \\
\text { solved } \\
\text { (mg/L } \\
\text { as } \mathrm{Ca} \text { ) }\end{array}$ & $\begin{array}{l}\text { Magne- } \\
\text { sium, } \\
\text { dis- } \\
\text { solved } \\
\text { (mg/L } \\
\text { as Mg) }\end{array}$ & $\begin{array}{c}\text { Sodium, } \\
\text { dis- } \\
\text { solved } \\
\text { (mg/L } \\
\text { as } N a \text { ) }\end{array}$ \\
\hline $12-29-83$ & $16: 00$ & 37 & 5.7 & -- & 9 & 2.0 & 1.0 & 2.0 \\
\hline $01-30-84$ & $13: 30$ & 29 & 5.9 & 7.0 & 8 & 2.0 & .72 & 1.6 \\
\hline $02-29-84$ & $14: 30$ & -- & 5.8 & 7.5 & 8 & 2.2 & .70 & 1.4 \\
\hline $03-28-84$ & $13: 15$ & 25 & 5.7 & 6.0 & 7 & 1.9 & .58 & 1.2 \\
\hline $06-06-84$ & $15: 15$ & 28 & 5.9 & -- & 7 & 1.8 & .52 & 1.4 \\
\hline $07-17-84$ & $10: 30$ & 34 & 6.1 & 15.5 & 8 & 2.2 & .67 & 1.6 \\
\hline $02-19-85$ & $15: 00$ & 34 & 5.8 & 7.0 & 9 & 2.1 & .84 & 1.8 \\
\hline $03-19-85$ & $12: 30$ & 32 & 5.5 & 6.5 & 9 & 2.3 & .73 & 1.4 \\
\hline $04-10-85$ & $14: 30$ & 31 & 5.5 & 7.5 & 8 & 2.1 & .66 & 1.4 \\
\hline $08-20-85$ & $15: 30$ & 41 & 5.8 & 19.0 & 11 & 3.0 & .96 & 1.9 \\
\hline
\end{tabular}

\begin{tabular}{|c|c|c|c|c|c|c|c|c|c|c|}
\hline Date & $\begin{array}{l}\text { Potas- } \\
\text { sium, } \\
\text { dis- } \\
\text { solved } \\
\text { (mg/L } \\
\text { as K) }\end{array}$ & $\begin{array}{c}\text { Nitro- } \\
\text { gen, } \\
\text { ammonia } \\
\text { total } \\
\text { (mg/L } \\
\text { as N) }\end{array}$ & $\begin{array}{c}\text { Nitro- } \\
\text { gen, } \\
\text { ammonia, } \\
\text { dis- } \\
\text { solved } \\
\text { (mg/L } \\
\text { as N) }\end{array}$ & $\begin{array}{c}\text { Alka- } \\
\text { linity } \\
\text { field } \\
\text { (mg/L } \\
\text { as } \\
\mathrm{CaCO}_{3} \text { ) }\end{array}$ & $\begin{array}{l}\text { Sulfate, } \\
\text { dis- } \\
\text { solved } \\
\text { (mg/L } \\
\left.\text { as } \mathrm{SO}_{4}\right)\end{array}$ & $\begin{array}{c}\text { Nitro- } \\
\text { gen, } \\
\text { nitrate, } \\
\text { dis- } \\
\text { solved } \\
\text { (mg/L } \\
\text { as } N \text { ) }\end{array}$ & $\begin{array}{l}\text { Phos- } \\
\text { phorus, } \\
\text { ortho, } \\
\text { dis- } \\
\text { solved } \\
\text { (mg/L } \\
\text { as P) }\end{array}$ & $\begin{array}{l}\text { Chlo- } \\
\text { ride, } \\
\text { dis- } \\
\text { solved } \\
\text { (mg/L } \\
\text { as Cl) }\end{array}$ & $\begin{array}{l}\text { Fluo- } \\
\text { ride, } \\
\text { dis- } \\
\text { solved } \\
\text { (mg/L } \\
\text { as F) }\end{array}$ & $\begin{array}{c}\text { Silica, } \\
\text { dis- } \\
\text { solved } \\
\text { (mg/L } \\
\text { as } \\
\mathrm{SiO}_{2} \text { ) }\end{array}$ \\
\hline $\begin{array}{l}12-29-83 \\
01-30-84 \\
02-29-84 \\
03-28-84 \\
06-06-84\end{array}$ & $\begin{array}{r}0.86 \\
.61 \\
.60 \\
.49 \\
.71\end{array}$ & $\begin{aligned} &-- \\
&<0.001 \\
& .049 \\
& .012\end{aligned}$ & $\begin{array}{l}<0.01 \\
<.01 \\
-- \\
-- \\
--\end{array}$ & $\begin{array}{l}3 \\
2 \\
1 \\
1 \\
1\end{array}$ & $\begin{array}{l}12 \\
9.6 \\
10 \\
8.8 \\
9.9\end{array}$ & $\begin{array}{l}-- \\
-- \\
-- \\
--\end{array}$ & $\begin{array}{l}<0.06 \\
-- \\
-- \\
-- \\
--\end{array}$ & $\begin{array}{l}1.2 \\
1.2 \\
1.0 \\
.79 \\
.62\end{array}$ & $\begin{array}{r}<0.01 \\
.02 \\
.03 \\
<.01 \\
.05\end{array}$ & $\begin{array}{l}9.2 \\
8.6 \\
7.7 \\
6.9 \\
8.3\end{array}$ \\
\hline $\begin{array}{l}07-17-84 \\
02-19-85 \\
03-19-85 \\
04-10-85 \\
08-20-85\end{array}$ & $\begin{array}{l}.75 \\
.60 \\
.50 \\
.57 \\
.76\end{array}$ & $\begin{array}{l}.006 \\
\ldots \\
\ldots \\
-\infty\end{array}$ & $\begin{array}{r}- \\
<.02 \\
<.01 \\
<.01 \\
<.01\end{array}$ & $\begin{array}{l}2 \\
2 \\
2 \\
1 \\
4\end{array}$ & $\begin{array}{r}9.7 \\
.4 \\
7.8 \\
9.1 \\
12\end{array}$ & $\begin{array}{r}-- \\
<0.01 \\
.04 \\
<.01 \\
.01\end{array}$ & $\begin{array}{l}<-01 \\
<.01 \\
<.01 \\
<.01\end{array}$ & $\begin{array}{c}1.63 \\
.17 \\
1.1 \\
.87 \\
1.1\end{array}$ & $\begin{array}{r}5.01 \\
.73 \\
.10 \\
.01 \\
.07\end{array}$ & $\begin{array}{r}10 \\
9.0 \\
8.1 \\
8.3 \\
12\end{array}$ \\
\hline Date & $\begin{array}{l}\text { Alum- } \\
\text { inum, } \\
\text { dis- } \\
\text { solved } \\
(\mu \mathrm{g} / \mathrm{L} \\
\text { as Al) }\end{array}$ & $\begin{array}{l}\text { Copper, } \\
\text { dis- } \\
\text { solved } \\
\text { ( } \mu \mathrm{g} / \mathrm{L} \\
\text { as } \mathrm{Cu})\end{array}$ & $\begin{array}{l}\text { Iron, } \\
\text { dis- } \\
\text { solved } \\
(\mu \mathrm{g} / \mathrm{L} \\
\text { as } \mathrm{Fe})\end{array}$ & $\begin{array}{l}\text { Lead, } \\
\text { dis- } \\
\text { solved } \\
(\mu \mathrm{g} / \mathrm{L} \\
\text { as } \mathrm{Pb})\end{array}$ & $\begin{array}{l}\text { Manga- } \\
\text { nese, } \\
\text { dis- } \\
\text { solved } \\
(\mu g / L \\
\text { as } M n)\end{array}$ & $\begin{array}{c}\text { Mercury, } \\
\text { dis- } \\
\text { solved } \\
\text { ( } \mu \mathrm{g} / \mathrm{L} \\
\text { as } \mathrm{Hg} \text { ) }\end{array}$ & $\begin{array}{l}\text { Sele- } \\
\text { nium, } \\
\text { dis- } \\
\text { solved } \\
(\mu g / L \\
\text { as } \mathrm{Se})\end{array}$ & $\begin{array}{l}\text { Vana- } \\
\text { dium, } \\
\text { dis- } \\
\text { solved } \\
(\mu g / L \\
\text { as V) }\end{array}$ & $\begin{array}{l}\text { Carbon, } \\
\text { organic } \\
\text { total } \\
\text { (mg/L } \\
\text { as C) }\end{array}$ & $\begin{array}{c}\text { Carbon, } \\
\text { organic, } \\
\text { dis- } \\
\text { solved } \\
\text { (mg/L } \\
\text { as C) }\end{array}$ \\
\hline $\begin{array}{l}12-29-83 \\
01-30-84 \\
02-29-84 \\
03-28-84 \\
06-06-84\end{array}$ & $\begin{array}{r}<10 \\
30 \\
20 \\
60 \\
--\end{array}$ & $\begin{array}{r}2.1 \\
.9 \\
1.8 \\
1.8 \\
--\end{array}$ & $\begin{array}{r}220 \\
6 \\
17 \\
15 \\
47\end{array}$ & $\begin{array}{r}0.4 \\
1.6 \\
.3 \\
.5\end{array}$ & $\begin{array}{r}300 \\
50 \\
36 \\
23 \\
43\end{array}$ & $\begin{array}{r}<0.1 \\
.6 \\
<.1 \\
<.1 \\
\end{array}$ & $\begin{array}{l}<1 \\
<1 \\
<1 \\
<1 \\
--\end{array}$ & $\begin{array}{l}<1 \\
<1 \\
<1 \\
<1 \\
--\end{array}$ & $\begin{array}{l}-- \\
-- \\
1.6 \\
--\end{array}$ & $\begin{array}{c}1.6 \\
3.1 \\
-- \\
- \\
1.0\end{array}$ \\
\hline $\begin{array}{l}07-17-84 \\
02-19-85 \\
03-19-85 \\
04-10-85 \\
08-20-85\end{array}$ & $\begin{array}{l}10 \\
-- \\
-- \\
30 \\
30\end{array}$ & $\begin{array}{r}.4 \\
1.5 \\
-\quad .9 \\
3.0\end{array}$ & $\begin{array}{r}15 \\
510 \\
38 \\
94 \\
11\end{array}$ & $\begin{array}{r}.6 \\
.7 \\
-\quad \\
.1 \\
2.0\end{array}$ & $\begin{array}{l}48 \\
42 \\
23 \\
30 \\
24\end{array}$ & $\begin{array}{l}<.1 \\
-- \\
-- \\
-- \\
--\end{array}$ & $\begin{array}{l}<1 \\
-- \\
-- \\
-- \\
--\end{array}$ & $\begin{array}{l}<1 \\
-- \\
-- \\
-- \\
--\end{array}$ & $\begin{array}{l}-- \\
-- \\
-- \\
--\end{array}$ & $\begin{array}{l}-- \\
-- \\
-- \\
-- \\
--\end{array}$ \\
\hline
\end{tabular}

1 Anomalous value that contributed to cation-anion balance difference of greater than 10 percent; value discarded in data analysis. 
Table 9.--Physical characteristics and concentrations of chemical constituents of ground water at station number 422803072231801, Swift River sand and gravel well near Cooleyville, Massachusetts

$\left[\mu \mathrm{S} / \mathrm{cm}\right.$, microsiemens per centimeter at 25 degrees Celsius; ${ }^{\circ} \mathrm{C}$, degrees Celsius; $\mathrm{mg} / \mathrm{L}$, milligrams per liter;

$\mu \mathrm{g} / \mathrm{L}$, micrograms per liter, <, less than; dashes indicate not analyzed]

\begin{tabular}{|c|c|c|c|c|c|c|c|c|c|}
\hline Date & Time & $\begin{array}{l}\text { Spe- } \\
\text { cific } \\
\text { con- } \\
\text { duct- } \\
\text { ance } \\
(\mu \mathrm{s} / \mathrm{cm})\end{array}$ & $\begin{array}{l}\mathrm{pH} \\
\text { (stand- } \\
\text { ard } \\
\text { units) }\end{array}$ & $\begin{array}{l}\text { Temper- } \\
\text { ature } \\
\left({ }^{\circ} \mathrm{C}\right)\end{array}$ & $\begin{array}{l}\text { Hard- } \\
\text { ness } \\
\text { (mg/L } \\
\text { as } \\
\mathrm{CaCO}_{3} \text { ) }\end{array}$ & $\begin{array}{c}\text { Calcium, } \\
\text { dis- } \\
\text { solved } \\
\text { (mg/L } \\
\text { as } \mathrm{Ca} \text { ) }\end{array}$ & $\begin{array}{c}\text { Magne- } \\
\text { sium, } \\
\text { dis- } \\
\text { solved } \\
\text { (mg/L } \\
\text { as Mg) }\end{array}$ & $\begin{array}{l}\text { Sodium, } \\
\text { dis- } \\
\text { solved } \\
\text { (mg/L } \\
\text { as } \mathrm{Na} \text { ) }\end{array}$ & $\begin{array}{l}\text { Potas- } \\
\text { sium, } \\
\text { dis- } \\
\text { solved } \\
\text { (mg/L } \\
\text { as } K \text { ) }\end{array}$ \\
\hline $\begin{array}{l}12-20-83 \\
01-30-84 \\
02-28-84 \\
03-28-84 \\
04-25-84\end{array}$ & $\begin{array}{l}13: 45 \\
11: 30 \\
14: 45 \\
14: 45 \\
16: 00\end{array}$ & $\begin{array}{l}34 \\
33 \\
-- \\
30 \\
32\end{array}$ & $\begin{array}{l}-- \\
6.0 \\
5.8 \\
6.0 \\
5.9\end{array}$ & $\begin{array}{l}9.0 \\
7.5 \\
7.5 \\
7.0 \\
7.0\end{array}$ & $\begin{array}{l}9 \\
9 \\
8 \\
8 \\
8\end{array}$ & $\begin{array}{l}2.6 \\
2.6 \\
2.7 \\
2.5 \\
2.6\end{array}$ & $\begin{array}{r}0.50 \\
.49 \\
.42 \\
.49 \\
.44\end{array}$ & $\begin{array}{l}2.1 \\
2.0 \\
2.0 \\
1.9 \\
1.8\end{array}$ & $\begin{array}{r}0.84 \\
.81 \\
.78 \\
.78 \\
.75\end{array}$ \\
\hline $\begin{array}{l}04-25-84 \\
06-06-84 \\
07-17-84 \\
09-12-84 \\
10-24-84\end{array}$ & $\begin{array}{l}16: 15 \\
14: 00 \\
09: 30 \\
14: 00 \\
09: 30\end{array}$ & $\begin{array}{l}32 \\
29 \\
31 \\
24 \\
30\end{array}$ & $\begin{array}{l}5.9 \\
5.8 \\
5.7 \\
5.8 \\
5.8\end{array}$ & $\begin{array}{l}7.0 \\
-- \\
11.0 \\
9.0 \\
9.0\end{array}$ & $\begin{array}{l}8 \\
9 \\
9 \\
8 \\
8\end{array}$ & $\begin{array}{l}2.6 \\
2.6 \\
2.6 \\
2.5 \\
2.5\end{array}$ & $\begin{array}{l}.47 \\
.49 \\
.51 \\
.46 \\
.47\end{array}$ & $\begin{array}{l}1.9 \\
1.8 \\
1.8 \\
1.9 \\
2.1\end{array}$ & $\begin{array}{r}-- \\
.76 \\
.79 \\
.94 \\
.79\end{array}$ \\
\hline $\begin{array}{l}11-20-84 \\
12-20-84 \\
01-29-85 \\
02-19-85 \\
03-19-85\end{array}$ & $\begin{array}{l}13: 45 \\
08: 45 \\
13: 15 \\
14: 00 \\
12: 00\end{array}$ & $\begin{array}{l}31 \\
35 \\
32 \\
31 \\
31\end{array}$ & $\begin{array}{l}5.7 \\
5.7 \\
5.8 \\
5.7 \\
5.8\end{array}$ & $\begin{array}{l}7.0 \\
8.5 \\
7.5 \\
7.5 \\
6.0\end{array}$ & $\begin{array}{r}-- \\
8 \\
8 \\
8 \\
8\end{array}$ & $\begin{array}{r}-- \\
2.5 \\
2.5 \\
2.5 \\
2.5\end{array}$ & $\begin{array}{r}-- \\
.51 \\
.52 \\
.44 \\
.52\end{array}$ & $\begin{array}{l}-- \\
2.0 \\
1.9 \\
1.9 \\
1.9\end{array}$ & $\begin{array}{r}- \\
.77 \\
.85 \\
.76 \\
.73\end{array}$ \\
\hline $\begin{array}{l}04-10-85 \\
06-11-85 \\
08-20-85\end{array}$ & $\begin{array}{l}12: 45 \\
13: 30 \\
13: 50\end{array}$ & $\begin{array}{l}31 \\
31 \\
33\end{array}$ & $\begin{array}{l}5.8 \\
5.2 \\
5.7\end{array}$ & $\begin{array}{r}7.0 \\
8.0 \\
11.0\end{array}$ & $\begin{array}{l}8 \\
9 \\
8\end{array}$ & $\begin{array}{l}2.5 \\
2.7 \\
2.5\end{array}$ & $\begin{array}{l}.48 \\
.49 \\
.48\end{array}$ & $\begin{array}{l}1.9 \\
1.8 \\
1.9\end{array}$ & $\begin{array}{l}.75 \\
.74 \\
.75\end{array}$ \\
\hline
\end{tabular}

\begin{tabular}{|c|c|c|c|c|c|c|c|c|c|c|}
\hline Date & $\begin{array}{c}\text { Nitro- } \\
\text { gen, } \\
\text { ammonia } \\
\text { total } \\
\text { (mg/L } \\
\text { as N) }\end{array}$ & $\begin{array}{l}\text { Nitro- } \\
\text { gen, } \\
\text { ammonia, } \\
\text { dis- } \\
\text { solved } \\
\text { (mg/L } \\
\text { as N) }\end{array}$ & $\begin{array}{c}\text { Alka- } \\
\text { linity } \\
\text { field } \\
\text { (mg/L } \\
\text { as } \\
\mathrm{CaCO}_{3} \text { ) }\end{array}$ & $\begin{array}{l}\text { Sulfate, } \\
\text { dis- } \\
\text { solved } \\
\text { (mg/L } \\
\text { as } \mathrm{SO}_{4} \text { ) }\end{array}$ & $\begin{array}{c}\text { Nitro- } \\
\text { gen, } \\
\text { nitrate, } \\
\text { dis- } \\
\text { solved } \\
\text { (mg/L } \\
\text { as N) }\end{array}$ & $\begin{array}{l}\text { Phos- } \\
\text { phorus, } \\
\text { ortho, } \\
\text { dis- } \\
\text { solved } \\
\text { (mg/L } \\
\text { as P) }\end{array}$ & $\begin{array}{l}\text { Chlo- } \\
\text { ride, } \\
\text { dis- } \\
\text { solved } \\
\text { (mg/L } \\
\text { as Cl) }\end{array}$ & $\begin{array}{l}\text { Fluo- } \\
\text { ride, } \\
\text { dis- } \\
\text { solved } \\
\text { (mg/L } \\
\text { as F) }\end{array}$ & $\begin{array}{l}\text { Silica, } \\
\text { dis- } \\
\text { solved } \\
\text { (mg/L } \\
\text { as } \\
\mathrm{SiO}_{2} \text { ) }\end{array}$ & $\begin{array}{l}\text { Alum- } \\
\text { inum, } \\
\text { dis- } \\
\text { solved } \\
(\mu g / L \\
\text { as Al) }\end{array}$ \\
\hline $\begin{array}{l}12-20-83 \\
01-30-84 \\
02-28-84 \\
03-28-84 \\
04-25-84\end{array}$ & $\begin{aligned} &-- \\
&-- \\
&<0.001 \\
& .016 \\
& .049\end{aligned}$ & $\begin{array}{l}0.10 \\
<.01 \\
-- \\
-- \\
--\end{array}$ & $\begin{array}{r}6 \\
7 \\
7 \\
6 \\
--\end{array}$ & $\begin{array}{l}6.0 \\
6.3 \\
6.5 \\
6.2 \\
6.3\end{array}$ & $\begin{array}{l}<0.05 \\
-- \\
-- \\
-- \\
--\end{array}$ & $\begin{array}{l}<0.06 \\
-- \\
-- \\
-- \\
--\end{array}$ & $\begin{array}{l}1.2 \\
1.3 \\
1.3 \\
1.1 \\
1.2\end{array}$ & $\begin{array}{r}0.03 \\
.03 \\
.05 \\
<.01 \\
.04\end{array}$ & $\begin{array}{l}11 \\
11 \\
11 \\
10 \\
10\end{array}$ & $\begin{array}{r}20 \\
30 \\
20 \\
<10 \\
20\end{array}$ \\
\hline $\begin{array}{l}04-25-84 \\
06-06-84 \\
07-17-84 \\
09-12-84 \\
10-24-84\end{array}$ & $\begin{array}{r}-.059 \\
.019 \\
<.001 \\
--\end{array}$ & $\begin{array}{l}-- \\
-- \\
-- \\
-- \\
--\end{array}$ & $\begin{array}{l}7 \\
6 \\
6 \\
6 \\
5\end{array}$ & $\begin{array}{l}-- \\
7.2 \\
6.1 \\
6.2 \\
6.5\end{array}$ & $\begin{array}{ll}-- & \\
-- & \\
-- & \\
-- & \\
& .04\end{array}$ & $\begin{array}{l}-- \\
-- \\
-- \\
-- \\
\quad<.01\end{array}$ & $\begin{array}{c}-- \\
1.3 \\
1.2 \\
1.1 \\
1.2\end{array}$ & $\begin{array}{c}- \\
<.01 \\
.05 \\
.03 \\
.03\end{array}$ & $\begin{array}{l}10 \\
10 \\
10 \\
11 \\
11\end{array}$ & $\begin{array}{c}<10 \\
-- \\
10 \\
90 \\
30\end{array}$ \\
\hline $\begin{array}{l}11-20-84 \\
12-20-84 \\
01-29-85 \\
02-19-85 \\
03-19-85\end{array}$ & $\begin{array}{l}<.001 \\
<.001 \\
-- \\
-- \\
--\end{array}$ & $\begin{array}{r}-- \\
-04 \\
.04 \\
.05 \\
<.01\end{array}$ & $\begin{array}{l}5 \\
5 \\
6 \\
6 \\
6\end{array}$ & $\begin{array}{r}-- \\
6.0 \\
5.8 \\
+6.3 \\
-1\end{array}$ & $\begin{array}{r}-04 \\
.02 \\
.04 \\
<.01\end{array}$ & $\begin{array}{l}<.01 \\
<.01 \\
<.01 \\
<.01\end{array}$ & $\begin{array}{c}-- \\
1.1 \\
.58 \\
1.1 \\
2.8\end{array}$ & $\begin{array}{r}-02 \\
.01 \\
.04 \\
.06\end{array}$ & $\begin{array}{l}-- \\
11 \\
11 \\
11 \\
10\end{array}$ & $\begin{array}{r}-- \\
<10 \\
90 \\
30 \\
30\end{array}$ \\
\hline $\begin{array}{l}04-10-85 \\
06-11-85 \\
08-20-85\end{array}$ & $\begin{array}{l}-- \\
-- \\
--\end{array}$ & $\begin{array}{r}<.01 \\
.03 \\
<.01\end{array}$ & $\begin{array}{l}6 \\
5 \\
5\end{array}$ & $\begin{array}{l}6.2 \\
6.3 \\
7.4\end{array}$ & $\begin{array}{l}.03 \\
.03 \\
.04\end{array}$ & $\begin{array}{l}<.01 \\
<.01 \\
<.01\end{array}$ & $\begin{array}{l}1.2 \\
1.2 \\
1.3\end{array}$ & $\begin{array}{l}.02 \\
.01 \\
.05\end{array}$ & $\begin{array}{l}11 \\
10 \\
11\end{array}$ & $\begin{array}{l}30 \\
10 \\
40\end{array}$ \\
\hline
\end{tabular}


Table 9.--Physical characteristics and concentrations of chemical constituents of ground water at station number 422803072231801, Swift River sand and gravel well near Cooleyville, Massachusetts--Continued

\begin{tabular}{|c|c|c|c|c|c|c|c|c|c|c|}
\hline Date & $\begin{array}{c}\text { Arsenic, } \\
\text { total } \\
\text { ( } \mu g / L \\
\text { as As) }\end{array}$ & $\begin{array}{c}\text { Copper, } \\
\text { dis- } \\
\text { solved } \\
\text { ( } \mu \mathrm{g} / \mathrm{L} \\
\text { as } \mathrm{Cu})\end{array}$ & $\begin{array}{l}\text { Iron, } \\
\text { dis- } \\
\text { solved } \\
(\mu \mathrm{g} / \mathrm{L} \\
\mathrm{as} \mathrm{Fe})\end{array}$ & $\begin{array}{l}\text { Lead, } \\
\text { dis- } \\
\text { solved } \\
(\mu g / L \\
\text { as } \mathrm{Pb})\end{array}$ & $\begin{array}{l}\text { Manga- } \\
\text { nese, } \\
\text { dis- } \\
\text { solved } \\
\text { ( } \mu \text { g/L } \\
\text { as } M n \text { ) }\end{array}$ & $\begin{array}{c}\text { Mercury, } \\
\text { dis- } \\
\text { solved } \\
\text { ( } \mu \text { g/L } \\
\text { as Hg) }\end{array}$ & $\begin{array}{l}\text { Sele- } \\
\text { nium, } \\
\text { dis- } \\
\text { solved } \\
\text { ( } \mu \text { g/L } \\
\text { as Se) }\end{array}$ & $\begin{array}{l}\text { Vana- } \\
\text { dium, } \\
\text { dis- } \\
\text { solved } \\
(\mu \mathrm{g} / \mathrm{L} \\
\text { as V) }\end{array}$ & $\begin{array}{c}\text { Carbon, } \\
\text { organic } \\
\text { total } \\
\text { (mg/L } \\
\text { as C) }\end{array}$ & $\begin{array}{c}\text { Carbon, } \\
\text { organic, } \\
\text { dis- } \\
\text { solved } \\
\text { (mg/L } \\
\text { as C) }\end{array}$ \\
\hline $12-20-83$ & -- & 0.1 & 10 & 0.1 & 19 & $<0.1$ & $<1$ & 1 & -- & 0.6 \\
\hline $01-30-84$ & -- & 1.0 & $<3$ & 1.8 & 5 & $<.1$ & $<1$ & $<1$ & -- & 3.5 \\
\hline $02-28-84$ & -- & 2.4 & 4 & .3 & 7 & $<.1$ & $<1$ & 1 & 0.9 & -- \\
\hline $03-28-84$ & -- & 3.2 & 8 & 1.5 & 5 & $<.1$ & $<\overline{1}$ & $<1$ & .7 & -- \\
\hline $04-25-84$ & -- & 2.8 & 12 & .2 & 5 & $<.1$ & $<1$ & $<1$ & -- & 1.0 \\
\hline $04-25-84$ & -- & -- & 20 & -- & 5 & -- & -- & -- & -- & -- \\
\hline $06-06-84$ & -- & -- & 5 & -- & 4 & -- & -- & -- & -- & .4 \\
\hline $07-17-84$ & -- & 1.6 & 5 & 1.5 & 5 & $<.1$ & $<1$ & $<1$ & -- & .5 \\
\hline $09-12-84$ & 1 & .2 & 81 & .1 & 5 & $<.1$ & $<1$ & $<1$ & -- & .2 \\
\hline $10-24-84$ & -- & .2 & 3 & .1 & 4 & $<.1$ & $<1$ & $<1$ & -- & .4 \\
\hline $11-20-84$ & -- & -- & -- & -- & -- & -- & -- & -- & -- & -- \\
\hline $12-20-84$ & -- & 1.4 & 4 & 1.0 & 5 & -- & -- & -- & -- & -- \\
\hline $01-29-85$ & -- & 1.0 & 4 & .1 & 4 & -- & -- & -- & -- & -- \\
\hline $02-19-85$ & -- & 1.6 & 4 & .1 & 5 & -- & -- & -- & -- & -- \\
\hline $03-19-85$ & -- & -- & 6 & -- & 4 & -- & -- & -- & -- & -- \\
\hline $04-10-85$ & -- & .1 & 5 & .2 & 4 & -- & -- & -- & -- & -- \\
\hline $06-11-85$ & -- & -- & 3 & -- & 5 & -- & -- & -- & -- & -- \\
\hline $08-20-85$ & -- & 1.0 & $<3$ & 1.0 & 4 & -- & -- & -- & -- & -- \\
\hline
\end{tabular}

1 Anomalous value that contributed to cation-anion balance difference of greater than 10 percent; value discarded in data analysis. 
Table 10.--Daily mean discharge at station number 01174050, East Branch Fever Brook near Petersham, Massachusetts

[Period of record begins November 29, 1983; $\left(\mathrm{ft}^{3} / \mathrm{s}\right) \mathrm{mi}^{2}$, cubic feet per second per square mile; dashes indicate not applicable]

Discharge, in cubic feet per second, water year october 1983 to September 1984

\begin{tabular}{|c|c|c|c|c|c|c|c|c|c|c|c|c|}
\hline Day & oct & Nov & Dec & Jan & Feb & Mar & Apr & May & June & July & Aug & Sept \\
\hline 1 & -- & -- & 16 & 8.3 & 4.0 & 12 & 13 & 7.0 & 130 & 1.4 & 2.1 & 0.44 \\
\hline 2 & -- & -- & 12 & 8.2 & 3.7 & 9.0 & 17 & 6.2 & 93 & 1.2 & 1.9 & .19 \\
\hline 3 & -- & -- & 11 & 7.8 & 4.0 & 7.9 & 19 & 6.2 & 61 & 1.1 & 1.8 & .27 \\
\hline 4 & -- & -- & 9.1 & 7.3 & 14 & 7.3 & 19 & 17 & 42 & 1.1 & 1.2 & .29 \\
\hline 5 & -- & -- & 9.9 & 7.1 & 17 & 6.5 & 43 & 23 & 28 & 1.1 & 1.1 & .19 \\
\hline 6 & -- & -- & 11 & 7.2 & 18 & 11 & 102 & 18 & 21 & 1.1 & 1.1 & .21 \\
\hline 7 & -- & -- & 26 & 7.3 & 12 & 11 & 62 & 15 & 22 & 11 & .97 & .19 \\
\hline 8 & -- & -- & 17 & 7.2 & 11 & 7.7 & 36 & 13 & 17 & 17 & .77 & .18 \\
\hline 9 & -- & -- & 14 & 6.5 & 11 & 6.8 & 28 & 21 & 12 & 8.3 & .68 & .21 \\
\hline 10 & -- & -- & 13 & 6.9 & 8.2 & 6.1 & 25 & 16 & 10 & 8.7 & .61 & .24 \\
\hline 11 & -- & -- & 7.5 & 7.1 & 10 & 5.3 & 23 & 14 & 8.7 & 7.6 & .60 & .22 \\
\hline 12 & -- & -- & 6.6 & 6.8 & 13 & 3.9 & 17 & 12 & 5.1 & 4.9 & .48 & .12 \\
\hline 13 & -- & -- & 21 & 5.0 & 14 & 3.2 & 15 & 17 & 5.1 & 2.2 & .45 & .21 \\
\hline 14 & -- & -- & 49 & 6.4 & 13 & 6.8 & 13 & 22 & 4.0 & 1.8 & .54 & .36 \\
\hline 15 & -- & -- & 39 & 5.1 & 25 & 5.7 & 17 & 19 & 2.0 & 1.6 & .70 & .33 \\
\hline 16 & -- & -- & 22 & 5.0 & 97 & 5.8 & 29 & 14 & 2.7 & 3.0 & .28 & .35 \\
\hline 17 & -- & -- & 13 & 4.1 & 60 & 5.9 & 35 & 14 & 2.7 & 4.5 & .32 & .39 \\
\hline 18 & -- & -- & 10 & 3.6 & 46 & 5.1 & 22 & 14 & 2.7 & 23 & .55 & .44 \\
\hline 19 & -- & -- & 10 & 5.4 & 39 & 9.3 & 16 & 13 & 3.4 & 30 & .42 & .44 \\
\hline 20 & -- & -- & 8.3 & 3.3 & 38 & 14 & 17 & 12 & 2.2 & 14 & 1.1 & .47 \\
\hline 21 & -- & -- & 6.0 & 3.3 & 29 & 16 & 17 & 12 & 1.6 & 12 & .82 & .22 \\
\hline 22 & -- & -- & 9.3 & 3.7 & 21 & 26 & 15 & 9.1 & 1.3 & 8.9 & .94 & .31 \\
\hline 23 & - & -- & 12 & 3.3 & 17 & 28 & 14 & 8.7 & 1.1 & 6.8 & 1.1 & .26 \\
\hline 24 & -- & -- & 11 & 5.0 & 19 & 20 & 14 & 9.2 & .87 & 5.8 & .41 & .22 \\
\hline 25 & -- & -- & 9.0 & 12 & 21 & 16 & 14 & 8.5 & 1.4 & 2.0 & .31 & .21 \\
\hline 26 & -- & -- & 9.0 & 11 & 15 & 16 & 14 & 7.7 & 1.9 & 1.8 & .23 & .27 \\
\hline 27 & -- & -- & 7.7 & 9.7 & 14 & 14 & 12 & 7.8 & 1.6 & 2.2 & .21 & .23 \\
\hline 28 & -- & -- & 9.2 & 7.5 & 16 & 16 & 10 & 5.1 & 1.5 & 2.1 & .17 & .28 \\
\hline 29 & $\ldots$ & 30 & 20 & 5.2 & 20 & 18 & 8.6 & 45 & 1.3 & 1.6 & .20 & .43 \\
\hline 30 & -- & 25 & 14 & 6.1 & - & 17 & 9.0 & 200 & 1.2 & 2.1 & .25 & .47 \\
\hline 31 & -- & -- & 9.7 & 6.1 & -- & 15 & - & 185 & -- & 2.5 & .33 & -- \\
\hline Total & -- & -- & 442.3 & 198.5 & 629.9 & 352.3 & 695.6 & 791.5 & 488.37 & 192.4 & 22.64 & 8.64 \\
\hline Mean & -- & -- & 14.3 & 6.40 & 21.7 & 11.4 & 23.2 & 25.5 & 16.3 & 6.21 & .73 & .29 \\
\hline Maximum & - & -- & 49 & 12 & 97 & 28 & 102 & 200 & 130 & 30 & 2.1 & .47 \\
\hline Minjimum & - & -- & 6.0 & 3.3 & 3.7 & 3.2 & 8.6 & 5.1 & .87 & 1.1 & .17 & .12 \\
\hline$\left(f t^{3} / s\right) m i^{2}$ & 2 & -- & 2.57 & 1.15 & 3.91 & 2.04 & 4.17 & 4.59 & 2.93 & 1.12 & .13 & .05 \\
\hline Inches & -- & -- & 2.96 & 1.33 & 4.21 & 2.36 & 4.65 & 5.30 & 3.27 & 1.29 & .15 & .06 \\
\hline
\end{tabular}


Discharge, in cubic feet per second, water year October 1984 to september 1985

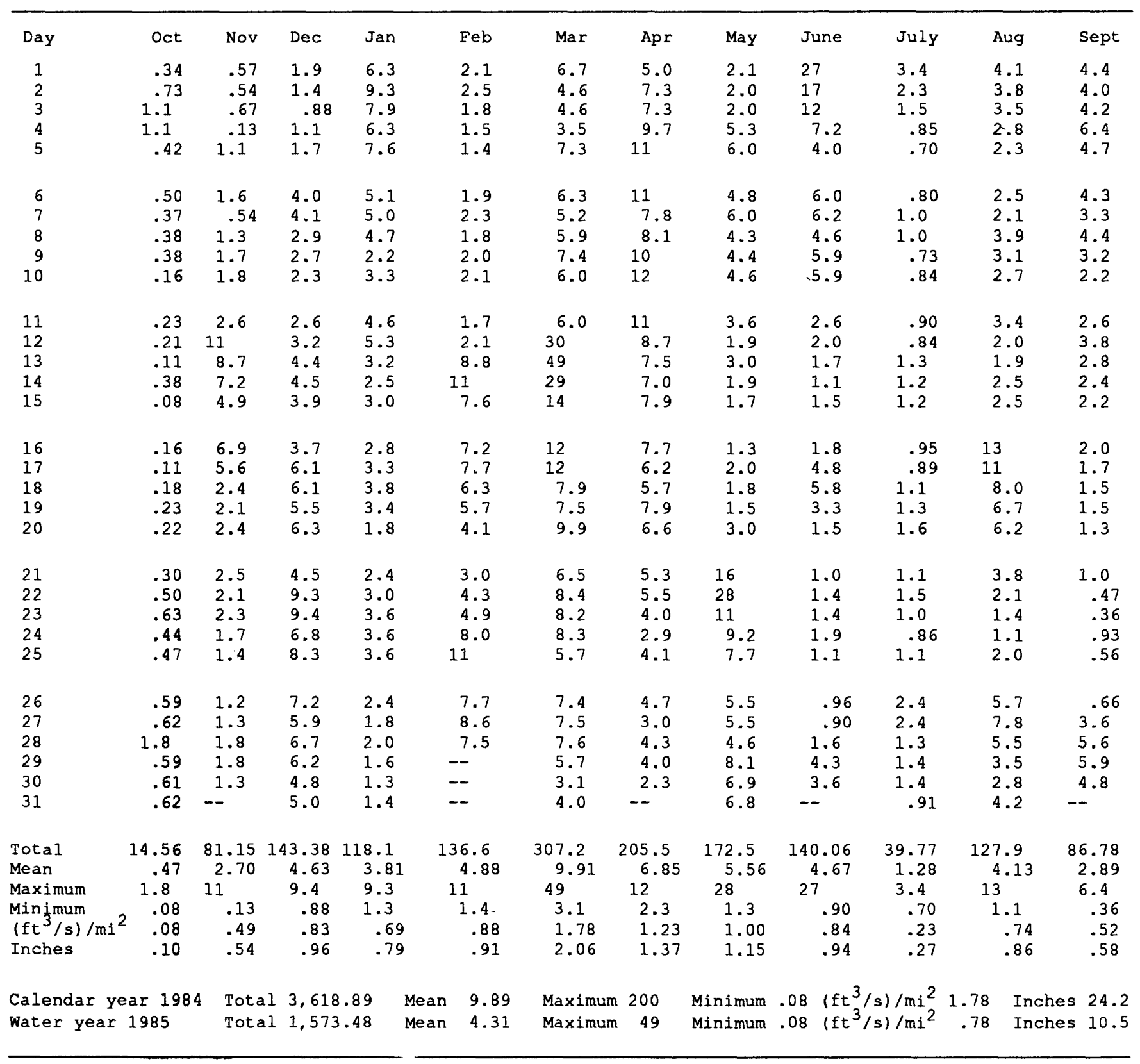


Table 11.--Physical characteristics and concentrations of chemical constituents of streamflow at station number 01174050, East Branch Fever Brook near Petersham, Massachusetts

$\left[\mathrm{ft}^{3} / \mathrm{s}\right.$, cubic feet per second; $\mu \mathrm{S} / \mathrm{cm}$, microsiemens per centimeter at 25 degrees Celsius; ${ }^{\circ} \mathrm{C}$, degrees Celsius; $\mathrm{mg} / \mathrm{L}$,milligrams per liter $; \mu \mathrm{g} / \mathrm{L}$, micrograms per liter, <, less than; dashes indicate not analyzed]

\begin{tabular}{|c|c|c|c|c|c|c|c|c|c|}
\hline Date & Time & $\begin{array}{l}\text { Stream- } \\
\text { flow, } \\
\text { instan- } \\
\text { taneous } \\
\left(\mathrm{ft}^{3} / \mathrm{s}\right)\end{array}$ & $\begin{array}{l}\text { Spe- } \\
\text { cific } \\
\text { con- } \\
\text { duct- } \\
\text { ance } \\
(\mu \mathrm{s} / \mathrm{cm})\end{array}$ & $\begin{array}{l}\mathrm{pH} \\
\text { (stand- } \\
\text { ard } \\
\text { units) }\end{array}$ & $\begin{array}{l}\text { Temper- } \\
\text { ature } \\
\left({ }^{\circ} \mathrm{C}\right)\end{array}$ & $\begin{array}{l}\text { Hard- } \\
\text { ness } \\
\text { (mg/L } \\
\text { as } \\
\mathrm{CaCO}_{3} \text { ) }\end{array}$ & $\begin{array}{l}\text { Calcium, } \\
\text { dis- } \\
\text { solved } \\
\text { (mg/L } \\
\text { as } \mathrm{Ca} \text { ) }\end{array}$ & $\begin{array}{l}\text { Magne- } \\
\text { sium, } \\
\text { dis- } \\
\text { solved } \\
\text { (mg/L } \\
\text { as Mg) }\end{array}$ & $\begin{array}{l}\text { Sodium, } \\
\text { dis- } \\
\text { solved } \\
\text { (mg/L } \\
\text { as } \mathrm{Na} \text { ) }\end{array}$ \\
\hline $\begin{array}{l}11-01-83 \\
11-08-83 \\
11-15-83 \\
11-22-83 \\
11-29-83\end{array}$ & $\begin{array}{l}15: 10 \\
14: 30 \\
13: 15 \\
15: 00 \\
09: 30\end{array}$ & $\begin{array}{r}1.0 \\
1.6 \\
3.0 \\
7.4 \\
22\end{array}$ & $\begin{array}{l}69 \\
67 \\
62 \\
62 \\
53\end{array}$ & $\begin{array}{l}6.4 \\
6.8 \\
6.2 \\
6.1 \\
5.7\end{array}$ & $\begin{array}{l}5.5 \\
6.0 \\
4.0 \\
6.5 \\
3.0\end{array}$ & $\begin{array}{r}13 \\
13 \\
12 \\
11 \\
8\end{array}$ & $\begin{array}{l}3.3 \\
3.3 \\
3.3 \\
2.9 \\
2.2\end{array}$ & $\begin{array}{l}1.1 \\
1.1 \\
1.0 \\
.91 \\
.72\end{array}$ & $\begin{array}{l}6.4 \\
6.0 \\
5.5 \\
6.0 \\
5.5\end{array}$ \\
\hline $\begin{array}{l}12-06-83 \\
12-13-83 \\
12-20-83 \\
12-27-83 \\
01-03-84\end{array}$ & $\begin{array}{l}09: 15 \\
13: 45 \\
10: 00 \\
09: 00 \\
08: 15\end{array}$ & $\begin{array}{l}9.2 \\
21 \\
8.2 \\
7.1 \\
8.9\end{array}$ & $\begin{array}{l}52 \\
43 \\
47 \\
58 \\
--\end{array}$ & $\begin{array}{l}5.7 \\
5.6 \\
5.7 \\
5.9 \\
5.9\end{array}$ & $\begin{array}{r}2.0 \\
2.0 \\
.5 \\
.5 \\
.5\end{array}$ & $\begin{array}{r}9 \\
9 \\
9 \\
8 \\
11\end{array}$ & $\begin{array}{l}2.4 \\
2.3 \\
2.3 \\
2.3 \\
2.9\end{array}$ & $\begin{array}{l}.76 \\
.74 \\
.69 \\
.53 \\
.99\end{array}$ & $\begin{array}{l}5.4 \\
4.2 \\
5.0 \\
5.8 \\
6.3\end{array}$ \\
\hline $\begin{array}{l}01-10-84 \\
01-17-84 \\
01-24-84 \\
01-27-84 \\
01-31-84\end{array}$ & $\begin{array}{l}08: 00 \\
14: 00 \\
14: 00 \\
17: 25 \\
14: 30\end{array}$ & $\begin{array}{c}6.3 \\
6.2 \\
4.6 \\
10 \\
5.0\end{array}$ & $\begin{array}{l}-- \\
57 \\
-- \\
74 \\
--\end{array}$ & $\begin{array}{l}5.9 \\
5.9 \\
6.0 \\
5.7 \\
6.2\end{array}$ & $--_{-.5}^{.5}$ & $\begin{array}{l}11 \\
10 \\
10 \\
11 \\
10\end{array}$ & $\begin{array}{l}2.9 \\
2.6 \\
2.7 \\
2.8 \\
2.7\end{array}$ & $\begin{array}{l}.91 \\
.83 \\
.86 \\
.93 \\
.89\end{array}$ & $\begin{array}{l}6.3 \\
5.8 \\
5.9 \\
8.1 \\
5.8\end{array}$ \\
\hline $\begin{array}{l}02-05-84 \\
02-07-84 \\
02-14-84 \\
02-15-84 \\
02-16-84\end{array}$ & $\begin{array}{l}13: 15 \\
14: 15 \\
09: 00 \\
15: 10 \\
07: 45\end{array}$ & $\begin{array}{l}17 \\
11 \\
13 \\
25 \\
97\end{array}$ & $\begin{array}{l}-- \\
-- \\
43 \\
50 \\
46\end{array}$ & $\begin{array}{l}6.0 \\
6.8 \\
5.8 \\
5.9 \\
5.6\end{array}$ & $\begin{array}{r}.5 \\
.5 \\
.5 \\
-- \\
--\end{array}$ & $\begin{array}{r}10 \\
10 \\
10 \\
9 \\
8\end{array}$ & $\begin{array}{l}2.5 \\
2.5 \\
2.4 \\
2.3 \\
1.9\end{array}$ & $\begin{array}{l}.83 \\
.82 \\
.87 \\
.79 \\
.77\end{array}$ & $\begin{array}{l}5.4 \\
6.2 \\
5.6 \\
5.0 \\
4.9\end{array}$ \\
\hline $\begin{array}{l}02-16-84^{2} \\
02-17-84 \\
02-21-84 \\
02-28-84 \\
03-06-84\end{array}$ & $\begin{array}{l}07: 50 \\
14: 00 \\
15: 45 \\
09: 00 \\
13: 45\end{array}$ & $\begin{array}{l}-- \\
60 \\
29 \\
15 \\
13\end{array}$ & $\begin{array}{l}-- \\
38 \\
44 \\
-- \\
--\end{array}$ & $\begin{array}{l}-- \\
5.7 \\
5.8 \\
5.7 \\
5.9\end{array}$ & $\begin{array}{r}- \\
.5 \\
.5 \\
.5 \\
.5\end{array}$ & $\begin{array}{r}-- \\
8 \\
8 \\
7 \\
8\end{array}$ & $\begin{array}{l}-- \\
2.0 \\
1.9 \\
2.0 \\
2.2\end{array}$ & $\begin{array}{r}- \\
.64 \\
.67 \\
.59 \\
.71\end{array}$ & $\begin{array}{l}-- \\
4.5 \\
4.9 \\
5.3 \\
5.5\end{array}$ \\
\hline $\begin{array}{l}03-13-84^{1} \\
03-20-84 \\
03-27-84 \\
03-27-84^{2} \\
04-03-84\end{array}$ & $\begin{array}{l}14: 00 \\
14: 00 \\
14: 15 \\
14: 20 \\
10: 30\end{array}$ & $\begin{array}{l}3.0 \\
16 \\
14 \\
-- \\
23\end{array}$ & $\begin{array}{l}48 \\
52 \\
41 \\
-- \\
49\end{array}$ & $\begin{array}{r}6.0 \\
5.9 \\
5.8 \\
-- \\
5.9\end{array}$ & $\begin{array}{r}.5 \\
1.0 \\
2.5 \\
-- \\
5.0\end{array}$ & $\begin{array}{r}10 \\
9 \\
7 \\
-- \\
7\end{array}$ & $\begin{array}{r}2.5 \\
2.3 \\
1.9 \\
-- \\
2.0\end{array}$ & $\begin{array}{r}.84 \\
.72 \\
.64 \\
-.6 \\
.60\end{array}$ & $\begin{array}{c}6.2 \\
5.8 \\
5.0 \\
-- \\
5.4\end{array}$ \\
\hline $\begin{array}{l}04-05-84 \\
04-06-84 \\
04-06-84^{2} \\
04-10-84 \\
04-10-84^{2}\end{array}$ & $\begin{array}{l}18: 30 \\
14: 00 \\
14: 10 \\
09: 15 \\
09: 20\end{array}$ & $\begin{array}{r}102 \\
59 \\
-- \\
23 \\
--\end{array}$ & $\begin{array}{l}31 \\
41 \\
-- \\
41 \\
--\end{array}$ & $\begin{array}{c}5.8 \\
5.7 \\
-- \\
5.7 \\
--\end{array}$ & $\begin{array}{c}-- \\
2.0 \\
-- \\
2.0 \\
--\end{array}$ & $\begin{array}{r}6 \\
6 \\
-- \\
6 \\
--\end{array}$ & $\begin{array}{c}1.7 \\
1.7 \\
-- \\
1.9 \\
--\end{array}$ & $\begin{array}{r}.54 \\
-.50 \\
-. \\
-.34\end{array}$ & $\begin{array}{r}3.9 \\
4.9 \\
-- \\
2.4 \\
--\end{array}$ \\
\hline $\begin{array}{l}04-17-84 \\
04-17-84^{2} \\
04-24-84 \\
05-01-84^{1} \\
05-08-84\end{array}$ & $\begin{array}{l}13: 30 \\
13: 40 \\
09: 30 \\
08: 45 \\
09: 00\end{array}$ & $\begin{array}{l}37 \\
-- \\
15 \\
6.3 \\
11\end{array}$ & $\begin{array}{l}43 \\
-- \\
52 \\
47 \\
52\end{array}$ & $\begin{array}{l}5.7 \\
-- \\
5.9 \\
6.3 \\
5.9\end{array}$ & $\begin{array}{l}6.5 \\
-- \\
10.0 \\
16.0 \\
15.0\end{array}$ & $\begin{array}{r}7 \\
-- \\
8 \\
8 \\
8\end{array}$ & $\begin{array}{l}1.8 \\
-- \\
2.0 \\
2.1 \\
2.0\end{array}$ & $\begin{array}{r}.56 \\
-. \\
.64 \\
.65 \\
.67\end{array}$ & $\begin{array}{c}4.6 \\
-- \\
5.1 \\
5.2 \\
5.6\end{array}$ \\
\hline
\end{tabular}

See footnotes at end of table. 


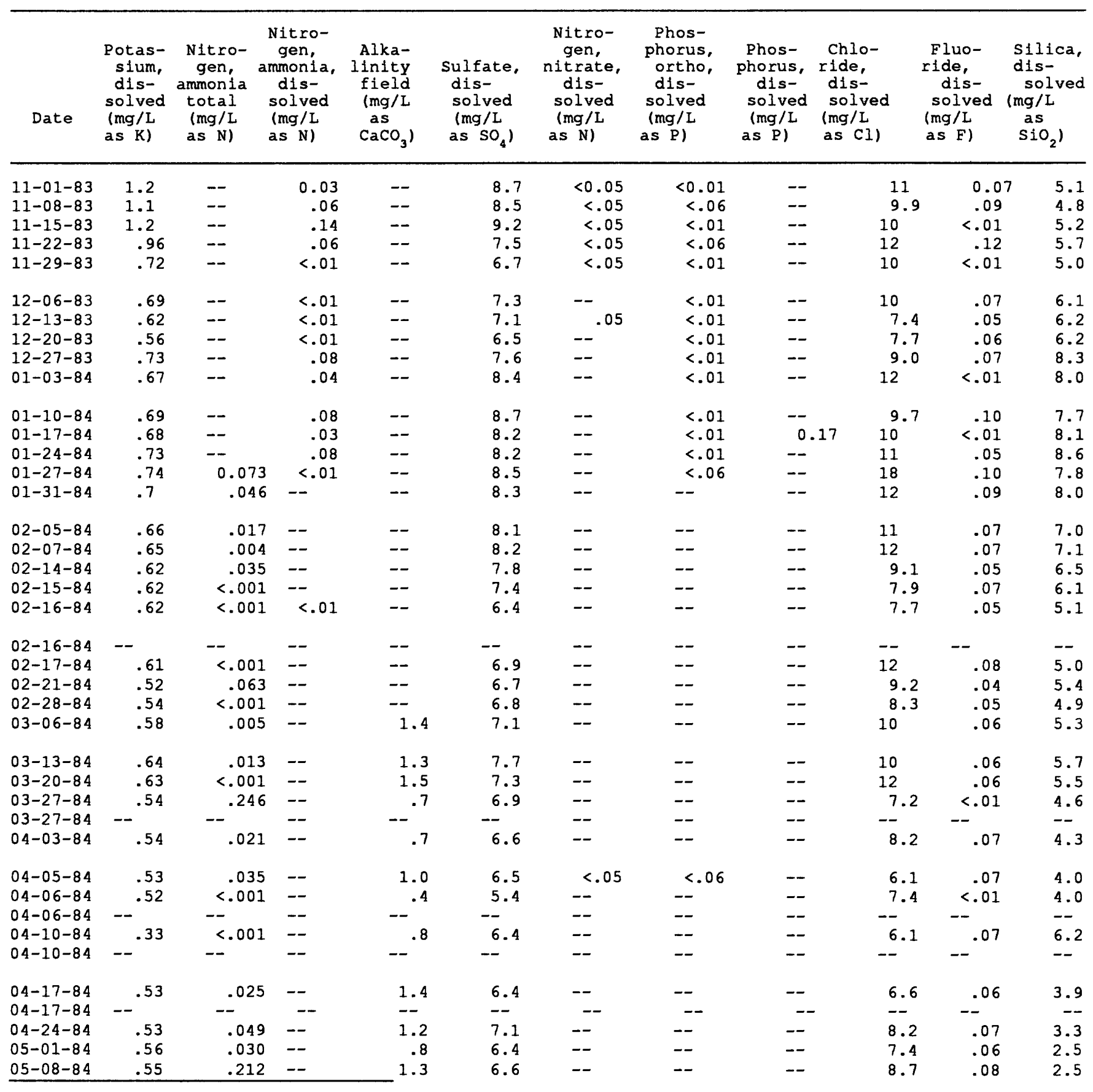

See footnotes at end of table. 
Table 11.--Physical characteristics and concentrations of chemical constituents of streamflow at station number 01174050, East Branch Fever Brook near Petersham, Massachusetts--Continued

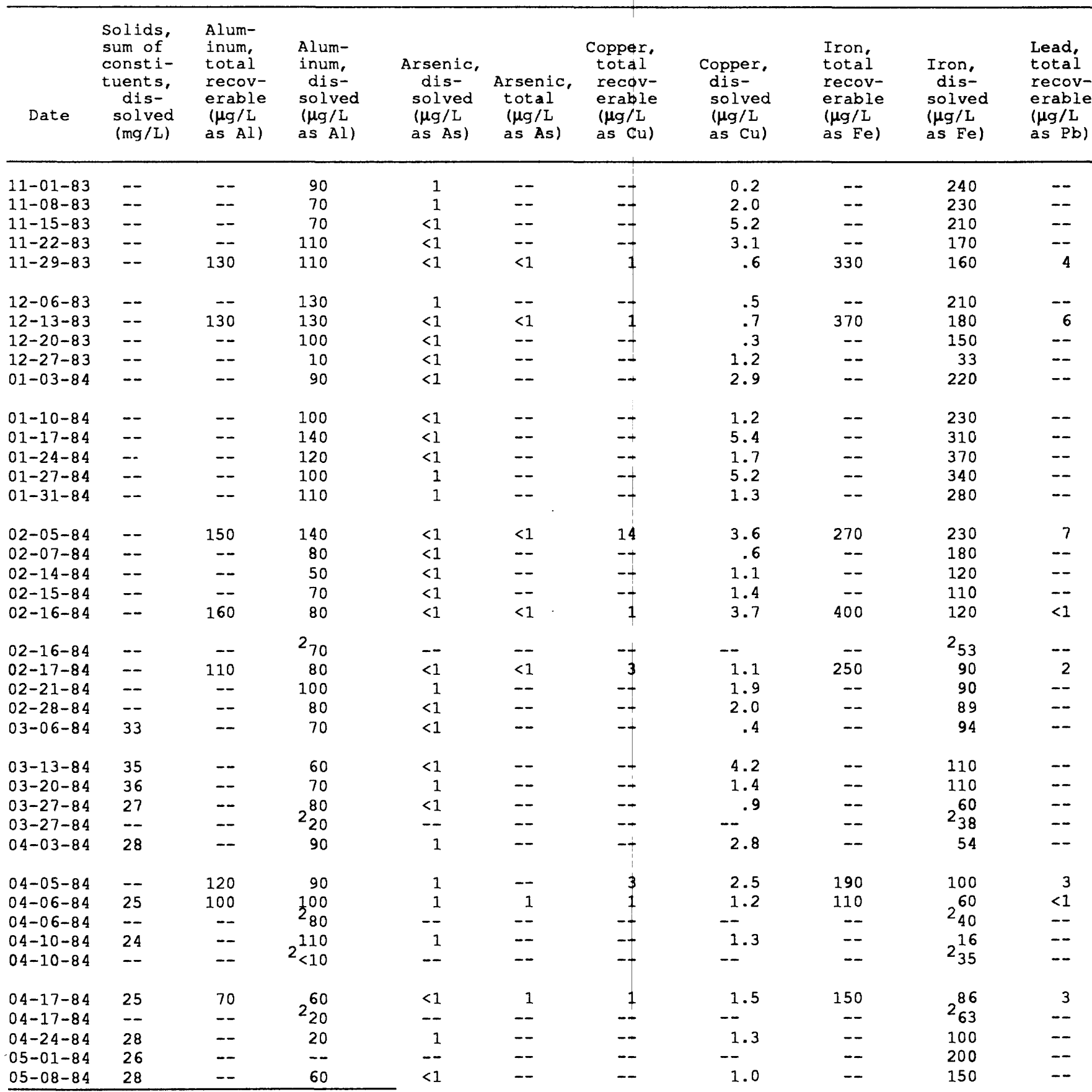

See footnotes at end of table. 
Table 11.--Physical characteristics and concentrations of chemical constituents of streamflow at station number 01174050, East Branch Fever Brook near Petersham, Massachusetts--Continued

\begin{tabular}{|c|c|c|c|c|c|c|c|c|c|c|}
\hline Date & $\begin{array}{l}\text { Lead, } \\
\text { dis- } \\
\text { solved } \\
(\mu g / L \\
\text { as } \mathrm{Pb})\end{array}$ & $\begin{array}{l}\text { Manga- } \\
\text { nese, } \\
\text { total } \\
\text { recov- } \\
\text { erable } \\
\text { ( } \mu g / L \\
\text { as } M n)\end{array}$ & $\begin{array}{l}\text { Manga- } \\
\text { nese, } \\
\text { dis- } \\
\text { solved } \\
\text { ( } \mu \mathrm{g} / \mathrm{L} \\
\text { as } \mathrm{Mn})\end{array}$ & $\begin{array}{l}\text { Mercury, } \\
\text { total } \\
\text { recov- } \\
\text { erable } \\
(\mu g / L \\
\text { as } \mathrm{Hg})\end{array}$ & $\begin{array}{c}\text { Mercury, } \\
\text { dis- } \\
\text { solved } \\
(\mu g / L \\
\text { as Hg) }\end{array}$ & $\begin{array}{l}\text { Sele- } \\
\text { nium, } \\
\text { total } \\
\text { ( } \mu g / L \\
\text { as Se) }\end{array}$ & $\begin{array}{l}\text { Sele- } \\
\text { nium, } \\
\text { dis- } \\
\text { solved } \\
(\mu g / L \\
\text { as } S e)\end{array}$ & $\begin{array}{l}\text { Vana- } \\
\text { dium, } \\
\text { dis- } \\
\text { solved } \\
(\mu g / L \\
\text { as V) }\end{array}$ & $\begin{array}{c}\text { Carbon, } \\
\text { organic } \\
\text { total } \\
\text { (mg/L } \\
\text { as C) }\end{array}$ & $\begin{array}{l}\text { Carbon, } \\
\text { organic, } \\
\text { dis- } \\
\text { solved } \\
\text { (mg/L } \\
\text { as C) }\end{array}$ \\
\hline $\begin{array}{l}11-01-83 \\
11-08-83 \\
11-15-83 \\
11-22-83 \\
11-29-83\end{array}$ & $\begin{array}{r}0.5 \\
.3 \\
.4 \\
1.1 \\
.4\end{array}$ & $\begin{array}{l}-- \\
-- \\
-- \\
50\end{array}$ & $\begin{array}{l}33 \\
35 \\
37 \\
51 \\
45\end{array}$ & $\begin{array}{l}-- \\
-- \\
-- \\
-- \\
<0.1\end{array}$ & $\begin{array}{r}<0.1 \\
.2 \\
.1 \\
.2 \\
<.1\end{array}$ & $\begin{array}{l}-- \\
-- \\
-- \\
-- \\
<1\end{array}$ & $\begin{array}{l}<1 \\
<1 \\
<1 \\
<1 \\
<1\end{array}$ & $\begin{array}{l}1 \\
1 \\
2 \\
2 \\
1\end{array}$ & $\begin{array}{l}-- \\
-- \\
-- \\
7.6 \\
7.5\end{array}$ & $\begin{array}{l}-- \\
-- \\
-- \\
-- \\
--\end{array}$ \\
\hline $\begin{array}{l}12-06-83 \\
12-13-83 \\
12-20-83 \\
12-27-83 \\
01-03-84\end{array}$ & $\begin{array}{r}.7 \\
.8 \\
1.0 \\
.3 \\
4.3\end{array}$ & $\begin{array}{l}-- \\
50 \\
-- \\
-- \\
--\end{array}$ & $\begin{array}{l}50 \\
45 \\
47 \\
43 \\
55\end{array}$ & $\begin{array}{l}-- \\
<.1 \\
-- \\
-- \\
--\end{array}$ & $\begin{array}{r}<.1 \\
<.1 \\
<.1 \\
.9 \\
<.1\end{array}$ & $\begin{array}{l}-- \\
<1 \\
-- \\
-- \\
--\end{array}$ & $\begin{array}{l}1 \\
<1 \\
<1 \\
<1 \\
<1\end{array}$ & $\begin{array}{r}<1 \\
<1 \\
1 \\
2 \\
1\end{array}$ & $\begin{array}{l}-- \\
6.0 \\
-- \\
-\end{array}$ & $\begin{array}{l}-- \\
-- \\
6.3 \\
5.9 \\
4.8\end{array}$ \\
\hline $\begin{array}{l}01-10-84 \\
01-17-84 \\
01-24-84 \\
01-27-84 \\
01-31-84\end{array}$ & $\begin{array}{r}4.4 \\
13.0 \\
2.9 \\
6.5 \\
1.9\end{array}$ & $\begin{array}{l}-- \\
-- \\
-- \\
-- \\
--\end{array}$ & $\begin{array}{l}52 \\
50 \\
53 \\
75 \\
53\end{array}$ & $\begin{array}{l}-- \\
-- \\
-- \\
-- \\
--\end{array}$ & $\begin{array}{l}<.1 \\
.2 \\
.2 \\
.1 \\
.3\end{array}$ & $\begin{array}{l}-- \\
-- \\
-- \\
--\end{array}$ & $\begin{array}{l}<1 \\
<1 \\
<1 \\
<1 \\
<1\end{array}$ & $\begin{array}{r}1 \\
<1 \\
<1 \\
<1 \\
1\end{array}$ & $\begin{array}{l}5.1 \\
-- \\
-- \\
-- \\
--\end{array}$ & $\begin{array}{l}-- \\
-- \\
-- \\
-- \\
6.7\end{array}$ \\
\hline $\begin{array}{l}02-05-84 \\
02-07-84 \\
02-14-84 \\
02-15-84 \\
02-16-84\end{array}$ & $\begin{array}{r}1.1 \\
.4 \\
1.3 \\
1.0 \\
1.0\end{array}$ & $\begin{array}{l}50 \\
-- \\
-- \\
-- \\
60\end{array}$ & $\begin{array}{l}57 \\
53 \\
41 \\
41 \\
68\end{array}$ & $\begin{array}{l}<.1 \\
-- \\
-- \\
--1\end{array}$ & $\begin{array}{r}<.1 \\
.3 \\
<.1 \\
.1 \\
.2\end{array}$ & $\begin{array}{l}<1 \\
-- \\
-- \\
-- \\
<1\end{array}$ & $\begin{array}{l}<1 \\
<1 \\
<1 \\
<1 \\
<1\end{array}$ & $\begin{array}{r}<1 \\
<1 \\
2 \\
1 \\
1\end{array}$ & $\begin{array}{l}-- \\
-- \\
-- \\
-- \\
--\end{array}$ & $\begin{array}{l}6.2 \\
4.0 \\
3.4 \\
3.9 \\
4.2\end{array}$ \\
\hline $\begin{array}{l}02-16-84 \\
02-17-84 \\
02-21-84 \\
02-28-84 \\
03-06-84\end{array}$ & $\begin{array}{r}-1.2 \\
.6 \\
1.0 \\
1.0\end{array}$ & $\begin{array}{l}-- \\
90 \\
-- \\
--\end{array}$ & $\begin{array}{l}28 \\
91 \\
58 \\
34 \\
31\end{array}$ & $\begin{array}{l}-- \\
<.1 \\
-- \\
--\end{array}$ & $\begin{array}{r}- \\
<.1 \\
<.1 \\
<.1 \\
.1\end{array}$ & $\begin{array}{l}-- \\
<1 \\
-- \\
-- \\
--\end{array}$ & $\begin{array}{l}-- \\
<1 \\
<1 \\
<1 \\
<1\end{array}$ & $\begin{array}{r}-- \\
2 \\
<1 \\
<1 \\
<1\end{array}$ & $\begin{array}{l}-- \\
-- \\
-- \\
4.2 \\
4.5\end{array}$ & $\begin{array}{l}-- \\
5.0 \\
4.3 \\
-- \\
--\end{array}$ \\
\hline $\begin{array}{l}03-13-84 \\
03-20-84 \\
03-27-84 \\
03-27-84 \\
04-03-84\end{array}$ & $\begin{array}{r}1.2 \\
.9 \\
.7 \\
-1.0\end{array}$ & $\begin{array}{l}-- \\
-- \\
-- \\
-- \\
--\end{array}$ & $\begin{array}{r}33 \\
37 \\
25 \\
25 \\
21\end{array}$ & $\begin{array}{l}-- \\
-- \\
-- \\
-- \\
--\end{array}$ & $\begin{array}{r}\quad .1 \\
<.1 \\
<.1 \\
-\quad \\
<.1\end{array}$ & $\begin{array}{l}-- \\
-- \\
-- \\
--\end{array}$ & $\begin{array}{l}<1 \\
<1 \\
<1 \\
-- \\
<1\end{array}$ & $\begin{array}{l}<1 \\
<1 \\
<1 \\
-- \\
<1\end{array}$ & $\begin{array}{r}3.9 \\
3.6 \\
3.0 \\
-- \\
2.3\end{array}$ & $\begin{array}{l}-- \\
-- \\
-- \\
-- \\
--\end{array}$ \\
\hline $\begin{array}{l}04-05-84 \\
04-06-84 \\
04-06-84 \\
04-10-84 \\
04-10-84\end{array}$ & $\begin{array}{r}1.1 \\
.9 \\
-- \\
-.\end{array}$ & $\begin{array}{l}20 \\
40 \\
-- \\
-- \\
--\end{array}$ & $\begin{array}{r}25 \\
34 \\
234 \\
34 \\
226\end{array}$ & $\begin{array}{l}-- \\
<.1 \\
-- \\
-- \\
--\end{array}$ & $\begin{array}{l}<.1 \\
<.1 \\
-- \\
<.1 \\
--\end{array}$ & $\begin{array}{l}-- \\
<1 \\
-- \\
-- \\
--\end{array}$ & $\begin{array}{l}<1 \\
<1 \\
-- \\
<1 \\
--\end{array}$ & $\begin{array}{l}1 \\
<1 \\
-< \\
<1 \\
--\end{array}$ & $\begin{array}{l}-- \\
3.6 \\
-- \\
--\end{array}$ & $\begin{array}{l}3.0 \\
-- \\
--\end{array}$ \\
\hline $\begin{array}{l}04-17-84 \\
04-17-84 \\
04-24-84 \\
05-01-84 \\
05-08-84\end{array}$ & $\begin{array}{c}.6 \\
-1.1 \\
-1.2\end{array}$ & $\begin{array}{l}30 \\
-- \\
-- \\
-- \\
--\end{array}$ & $\begin{array}{r}26 \\
26 \\
31 \\
38 \\
32\end{array}$ & $\begin{array}{l}<.1 \\
-- \\
-- \\
-- \\
--\end{array}$ & $\begin{array}{l}<.1 \\
-- \\
<.1 \\
-- \\
<.1\end{array}$ & $\begin{array}{l}<1 \\
-- \\
-- \\
-- \\
--\end{array}$ & $\begin{array}{l}<1 \\
-- \\
<1 \\
-- \\
<1\end{array}$ & $\begin{array}{l}<1 \\
-- \\
<1 \\
-- \\
<1\end{array}$ & $\begin{array}{l}-- \\
-- \\
3.9 \\
--\end{array}$ & $\begin{array}{r}4.0 \\
-- \\
3.9 \\
4.3 \\
4.3\end{array}$ \\
\hline
\end{tabular}

See footnotes at end of table. 
Table 11.--Physical characteristics and concentrations of chemical constituents of streamflow at station number 01174050, East Branch Fever Brook near Petersham, Massachusetts--Continued

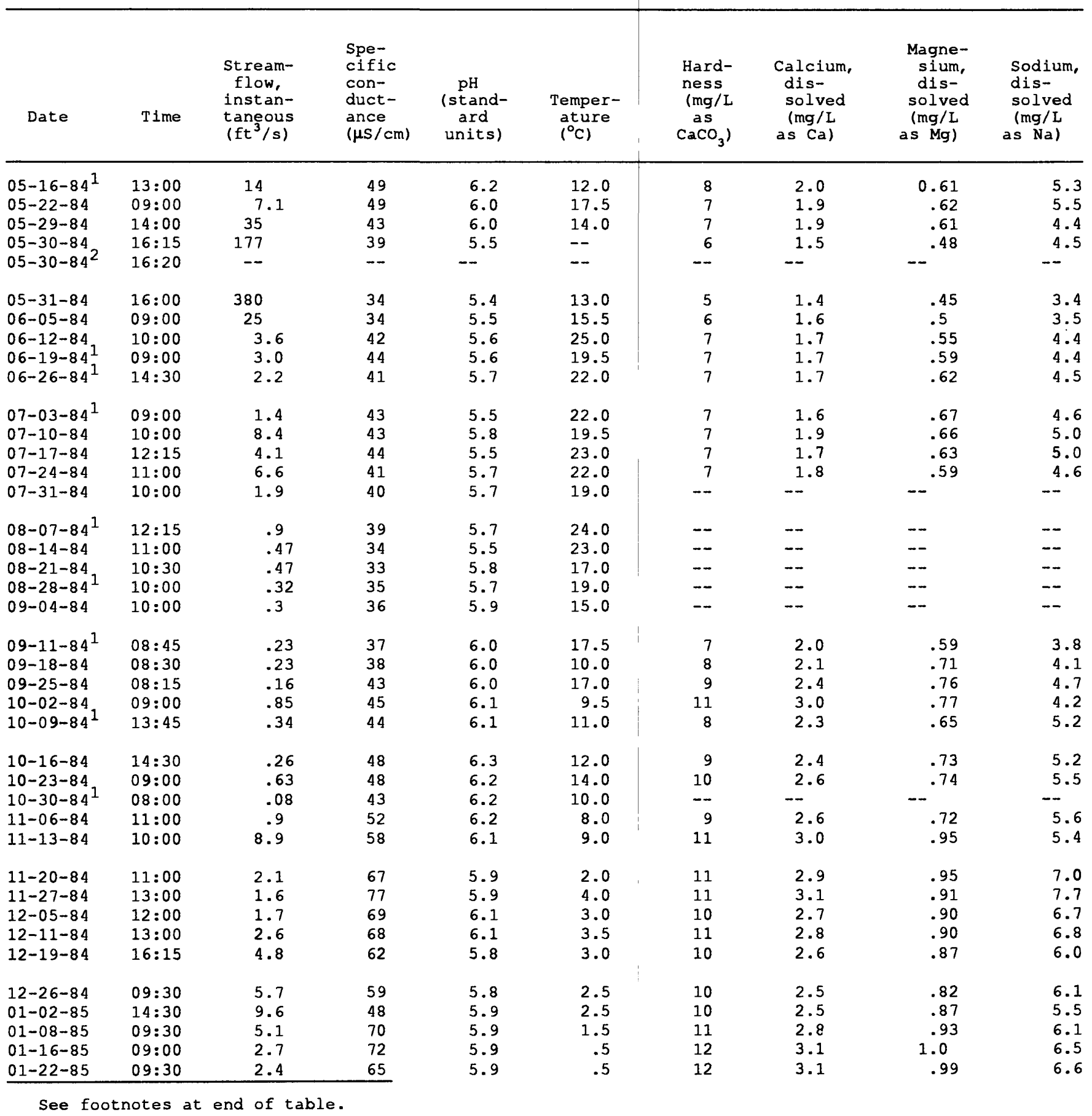


Table 11.--Physical characteristics and concentrations of chemical constituents of streamflow at station number 01174050, East Branch Fever Brook near Petersham, Massachusetts--Continued

\begin{tabular}{|c|c|c|c|c|c|c|c|c|c|c|}
\hline Date & $\begin{array}{l}\text { Potas- } \\
\text { sium, } \\
\text { dis- } \\
\text { solved } \\
\text { (mg/L } \\
\text { as K) }\end{array}$ & $\begin{array}{l}\text { Nitro- } \\
\text { gen, } \\
\text { ammonia } \\
\text { total } \\
\text { (mg/L } \\
\text { as N) }\end{array}$ & $\begin{array}{l}\text { Nitro- } \\
\text { gen, } \\
\text { ammonia, } \\
\text { dis- } \\
\text { solved } \\
\text { (mg/L } \\
\text { as N) }\end{array}$ & $\begin{array}{l}\text { Alka- } \\
\text { linity } \\
\text { field } \\
\text { (mg/L } \\
\mathrm{as} \\
\mathrm{CaCO}_{3} \text { ) }\end{array}$ & $\begin{array}{l}\text { Sulfate, } \\
\text { dis- } \\
\text { solved } \\
\text { (mg/L } \\
\text { as } \mathrm{SO}_{4} \text { ) }\end{array}$ & $\begin{array}{l}\text { Nitro- } \\
\text { gen, } \\
\text { nitrate, } \\
\text { dis- } \\
\text { solved } \\
\text { (mg/L } \\
\text { as N) }\end{array}$ & $\begin{array}{l}\text { Phos- } \\
\text { phorus, } \\
\text { ortho, } \\
\text { dis- } \\
\text { solved } \\
\text { (mg/L } \\
\text { as P) }\end{array}$ & $\begin{array}{l}\text { Chlo- } \\
\text { ride, } \\
\text { dis- } \\
\text { solved } \\
\text { (mg/L } \\
\text { as Cl) }\end{array}$ & $\begin{array}{l}\text { Fluo- } \\
\text { ride, } \\
\text { dis- } \\
\text { solved } \\
\text { (mg/L } \\
\text { as F) }\end{array}$ & $\begin{array}{l}\text { Silica, } \\
\text { dis- } \\
\text { solved } \\
\text { (mg/L } \\
\text { as } \\
\mathrm{SiO}_{2} \text { ) }\end{array}$ \\
\hline $\begin{array}{l}05-16-84 \\
05-22-84 \\
05-29-84 \\
05-30-84 \\
05-30-84\end{array}$ & $\begin{array}{r}0.54 \\
.45 \\
.56 \\
. .53\end{array}$ & $\begin{array}{r}0.252 \\
<.001 \\
.067 \\
.076\end{array}$ & $\begin{array}{l}-- \\
-- \\
-- \\
--\end{array}$ & $\begin{array}{r}0.8 \\
1.3 \\
1.5 \\
.6\end{array}$ & $\begin{array}{r}6.1 \\
6.5 \\
5.7 \\
4.4 \\
--\end{array}$ & $\begin{array}{l}-- \\
-- \\
-- \\
-- \\
--\end{array}$ & $\begin{array}{l}-- \\
-- \\
-- \\
-- \\
--\end{array}$ & $\begin{array}{l}7.9 \\
8.0 \\
7.3 \\
6.4 \\
--\end{array}$ & $\begin{array}{r}0.09 \\
.06 \\
.06 \\
.07\end{array}$ & $\begin{array}{r}2.9 \\
1.9 \\
2.9 \\
3.5 \\
--\end{array}$ \\
\hline $\begin{array}{l}05-31-84 \\
06-05-84 \\
06-12-84 \\
06-19-84 \\
06-26-84\end{array}$ & $\begin{array}{l}.48 \\
.35 \\
.47 \\
.52 \\
.46\end{array}$ & $\begin{array}{r}.068 \\
.027 \\
.080 \\
.008 \\
<.001\end{array}$ & $\begin{array}{l}-- \\
-- \\
-- \\
-- \\
--\end{array}$ & $\begin{array}{r}.2 \\
.6 \\
.9 \\
1.5 \\
1.7\end{array}$ & $\begin{array}{l}5.6 \\
6.3 \\
5.1 \\
4.9 \\
4.3\end{array}$ & $\begin{array}{l}<0.05 \\
-- \\
-- \\
-- \\
--\end{array}$ & $\begin{array}{l}-- \\
-- \\
-- \\
-- \\
--\end{array}$ & $\begin{array}{l}5.0 \\
4.8 \\
6.2 \\
6.8 \\
6.5\end{array}$ & $\begin{array}{l}.04 \\
.04 \\
.06 \\
.07 \\
.08\end{array}$ & $\begin{array}{l}4.1 \\
4.5 \\
4.1 \\
4.2 \\
3.9\end{array}$ \\
\hline $\begin{array}{l}07-03-84 \\
07-10-84 \\
07-17-84 \\
07-24-84 \\
07-31-84\end{array}$ & $\begin{array}{r}.48 \\
.58 \\
.45 \\
.37\end{array}$ & $\begin{array}{r}.032 \\
.075 \\
.015 \\
.058\end{array}$ & $\begin{array}{l}-- \\
-- \\
-- \\
-- \\
--\end{array}$ & $\begin{array}{r}1.4 \\
1.9 \\
.5 \\
1.4 \\
2.7\end{array}$ & $\begin{array}{r}4.0 \\
4.6 \\
4.0 \\
4.0 \\
--\end{array}$ & $\begin{array}{l}-- \\
-- \\
-- \\
-- \\
--\end{array}$ & $\begin{array}{l}-- \\
-- \\
-- \\
-- \\
--\end{array}$ & $\begin{array}{r}6.9 \\
7.4 \\
7.0 \\
6.5 \\
--\end{array}$ & $\begin{array}{r}.07 \\
.10 \\
.08 \\
.12 \\
-\end{array}$ & $\begin{array}{r}3.7 \\
4.1 \\
3.6 \\
3.8 \\
--\end{array}$ \\
\hline $\begin{array}{l}08-07-84 \\
08-14-84 \\
08-21-84 \\
08-28-84 \\
09-04-84\end{array}$ & $\begin{array}{l}-- \\
-- \\
-- \\
-- \\
--\end{array}$ & $\begin{array}{l}-- \\
-- \\
-- \\
-- \\
--\end{array}$ & $\begin{array}{l}-- \\
-- \\
-- \\
-- \\
--\end{array}$ & $\begin{array}{l}2.1 \\
2.2 \\
2.9 \\
3.1 \\
3.5\end{array}$ & $\begin{array}{l}-- \\
-- \\
-- \\
-- \\
--\end{array}$ & $\begin{array}{l}-- \\
-- \\
-- \\
-- \\
--\end{array}$ & $\begin{array}{l}-- \\
-- \\
-- \\
-- \\
--\end{array}$ & $\begin{array}{l}-- \\
-- \\
-- \\
-- \\
--\end{array}$ & $\begin{array}{l}-- \\
-- \\
-- \\
-- \\
--\end{array}$ & $\begin{array}{l}-- \\
-- \\
-- \\
-- \\
--\end{array}$ \\
\hline $\begin{array}{l}09-11-84 \\
09-18-84 \\
09-25-84 \\
10-02-84 \\
10-09-84\end{array}$ & $\begin{array}{c}.52 \\
.50 \\
.64 \\
1.0 \\
.71\end{array}$ & $\begin{array}{l}.037 \\
.170 \\
.190 \\
.033 \\
.028\end{array}$ & $\begin{array}{l}-- \\
-- \\
-- \\
-- \\
--\end{array}$ & $\begin{array}{l}3.8 \\
4.5 \\
4.4 \\
3.4 \\
4.3\end{array}$ & $\begin{array}{l}2.7 \\
2.9 \\
2.9 \\
6.0 \\
3.3\end{array}$ & $\begin{array}{r}-09 \\
.09 \\
.05 \\
.07\end{array}$ & $\begin{array}{l}-- \\
<0.01 \\
<.01 \\
<.01 \\
<.01\end{array}$ & $\begin{array}{l}5.4 \\
5.7 \\
6.3 \\
6.0 \\
7.1\end{array}$ & $\begin{array}{l}.07 \\
.09 \\
.06 \\
.07 \\
.09\end{array}$ & $\begin{array}{l}7.3 \\
7.8 \\
7.9 \\
8.0 \\
8.0\end{array}$ \\
\hline $\begin{array}{l}10-16-84 \\
10-23-84 \\
10-30-84 \\
11-06-84 \\
11-13-84\end{array}$ & $\begin{array}{c}.89 \\
1.4 \\
-- \\
1.1 \\
1.5\end{array}$ & $\begin{array}{r}.021 \\
.011 \\
.026 \\
<.001 \\
<.001\end{array}$ & $\begin{array}{l}-- \\
-- \\
-- \\
-- \\
--\end{array}$ & $\begin{array}{r}4.9 \\
5.5 \\
6.8 \\
-- \\
2.7\end{array}$ & $\begin{array}{l}3.8 \\
3.7 \\
- \\
4.2 \\
7.4\end{array}$ & $\begin{array}{l}.08 \\
-.10 \\
-- \\
--\end{array}$ & $\begin{array}{r}.01 \\
<.01 \\
-- \\
<.01 \\
<.01\end{array}$ & $\begin{array}{r}7.6 \\
7.6 \\
-- \\
8.0 \\
8.7\end{array}$ & $\begin{array}{r}<.01 \\
.07 \\
-\quad \\
.07 \\
.07\end{array}$ & $\begin{array}{r}8.0 \\
7.4 \\
-- \\
6.2 \\
4.9\end{array}$ \\
\hline $\begin{array}{l}11-20-84 \\
11-27-84 \\
12-05-84 \\
12-11-84 \\
12-19-84\end{array}$ & $\begin{array}{l}1.2 \\
1.2 \\
1.0 \\
1.0 \\
.85\end{array}$ & $\begin{array}{r}.006 \\
<.001 \\
.013 \\
.032 \\
.031\end{array}$ & $\begin{array}{l}-- \\
-- \\
-- \\
-- \\
--\end{array}$ & $\begin{array}{l}1.7 \\
2.9 \\
2.7 \\
2.1 \\
2.5\end{array}$ & $\begin{array}{l}7.5 \\
7.7 \\
7.3 \\
6.6 \\
6.8\end{array}$ & $\begin{array}{r}-- \\
<.01 \\
<.01 \\
.05 \\
--\end{array}$ & $\begin{array}{l}.02 \\
<.01 \\
<.01 \\
<.01 \\
<.01\end{array}$ & $\begin{array}{l}10 \\
13 \\
10 \\
10 \\
13\end{array}$ & $\begin{array}{r}<.01 \\
<.01 \\
.06 \\
.05\end{array}$ & $\begin{array}{l}5.0 \\
4.6 \\
4.1 \\
5.3 \\
5.7\end{array}$ \\
\hline $\begin{array}{l}12-26-84 \\
01-02-85 \\
01-08-85 \\
01-16-85 \\
01-22-85\end{array}$ & $\begin{array}{l}.85 \\
.77 \\
.85 \\
.92 \\
1.0\end{array}$ & $\begin{array}{l}-- \\
-- \\
-- \\
-- \\
--\end{array}$ & $\begin{array}{r}- \\
0.01 \\
.04 \\
.07 \\
.07\end{array}$ & $\begin{array}{l}1.4 \\
1.6 \\
2.0 \\
2.9 \\
3.3\end{array}$ & $\begin{array}{l}7.2 \\
6.4 \\
8.3 \\
7.9 \\
7.9\end{array}$ & $\begin{array}{l}.02 \\
.04 \\
.06 \\
.02 \\
.02\end{array}$ & $\begin{array}{l}<.01 \\
<.01 \\
.05 \\
<.01 \\
<.01\end{array}$ & $\begin{array}{l}10 \\
8.5 \\
11 \\
15 \\
27\end{array}$ & $\begin{array}{l}.04 \\
.06 \\
.09 \\
.05 \\
.13\end{array}$ & $\begin{array}{l}5.5 \\
5.6 \\
6.3 \\
7.2 \\
7.7\end{array}$ \\
\hline
\end{tabular}


Table 11.--Physical characteristics and concentrations of chemical constituents of streamflow at station number 01174050, East Branch Fever Brook near Petersham, Massachusetts--Continued

\begin{tabular}{|c|c|c|c|c|c|c|c|c|c|c|}
\hline Date & $\begin{array}{l}\text { Solids, } \\
\text { sum of } \\
\text { consti- } \\
\text { tuents, } \\
\text { dis- } \\
\text { solved } \\
\text { (mg/L) }\end{array}$ & $\begin{array}{l}\text { Alum- } \\
\text { inum, } \\
\text { total } \\
\text { recov- } \\
\text { erable } \\
(\mu g / L \\
\text { as Al) }\end{array}$ & $\begin{array}{l}\text { Alum- } \\
\text { inum, } \\
\text { dis- } \\
\text { solved } \\
(\mu g / L \\
\text { as Al) }\end{array}$ & $\begin{array}{c}\text { Arsenic, } \\
\text { dis- } \\
\text { solved } \\
\text { ( } \mu g / L \\
\text { as As) }\end{array}$ & $\begin{array}{l}\text { Arsenic, } \\
\text { total } \\
\text { ( } \mu \text { g/L } \\
\text { as As) }\end{array}$ & $\begin{array}{c}\text { Copper, } \\
\text { total } \\
\text { recov- } \\
\text { erable } \\
\text { ( } \mu g / L \\
\text { as C } 4)\end{array}$ & $\begin{array}{c}\text { Copper, } \\
\text { dis- } \\
\text { solved } \\
(\mu g / L \\
\text { as } \mathrm{Cu})\end{array}$ & $\begin{array}{l}\text { Iron, } \\
\text { total } \\
\text { recov- } \\
\text { erable } \\
(\mu g / L \\
\text { as } \mathrm{Fe})\end{array}$ & $\begin{array}{l}\text { Iron, } \\
\text { dis- } \\
\text { solved } \\
(\mu g / L \\
\text { as } \mathrm{Fe})\end{array}$ & $\begin{array}{l}\text { Lead, } \\
\text { total } \\
\text { recov- } \\
\text { erable } \\
(\mu g / L \\
\text { as } P b)\end{array}$ \\
\hline $05-16-84$ & 26 & -- & -- & -- & -- & -- & - & -- & 230 & -- \\
\hline $05-22-84$ & 26 & - & 70 & $<1$ & -- & -- & 6 & - & 150 & -- \\
\hline $05-29-84$ & 25 & -- & 70 & -- & -- & -- & -- & -- & 290 & -- \\
\hline $05-30-84$ & 22 & 130 & 110 & $<1$ & $<1$ & 1 & 2 & 340 & 190 & 2 \\
\hline $05-30-84$ & -- & -- & ${ }^{2} 80$ & -- & -- & -- & -- & -- & ${ }^{2} 120$ & -- \\
\hline $05-31-84$ & 21 & 150 & 150 & $<1$ & $<1$ & 3 & $<0.1$ & 420 & 140 & 3 \\
\hline $06-05-84$ & 22 & -- & -- & -- & -- & -- & -- & -- & 150 & -- \\
\hline $06-12-84$ & 24 & -- & 140 & $<1$ & -- & -- & 3.0 & -- & 350 & $\because$ \\
\hline $06-19-84$ & 25 & -- & -- & -- & -- & -- & -- & -- & 510 & -- \\
\hline $06-26-84$ & 24 & -- & 80 & $<1$ & -- & -- & 1.8 & -- & 540 & -- \\
\hline $07-03-84$ & 24 & -- & -- & -- & -- & -- & -- & -- & 660 & -- \\
\hline $07-10-84$ & 26 & -- & $<10$ & 1 & -- & -- & 1.4 & -- & 420 & -- \\
\hline $07-17-84$ & 23 & -- & -- & -- & -- & -- & -- & -- & 480 & -- \\
\hline $07-24-84$ & 23 & -- & 30 & 1 & -- & -- & 2.5 & - & 490 & -- \\
\hline $07-31-84$ & -- & -- & 110 & $<1$ & -- & -- & 3.2 & -- & - & -- \\
\hline $08-07-84$ & -- & -- & 70 & $<1$ & -- & -- & 7.5 & -- & -- & -- \\
\hline $08-14-84$ & -- & -- & 80 & 1 & -- & -- & 3.6 & -- & -- & -- \\
\hline $08-21-84$ & -- & -- & 70 & $<1$ & -- & -- & 1.2 & -- & -- & -- \\
\hline $08-28-84$ & -- & -- & 90 & $<1$ & -- & -- & .9 & -- & -- & -- \\
\hline $09-04-84$ & -- & -- & 80 & 1 & -- & -- & 5.1 & -- & -- & -- \\
\hline $09-11-84$ & -- & 90 & 60 & $<1$ & $<1$ & 1 & .9 & 1200 & 570 & $<1$ \\
\hline $09-18-84$ & 28 & -- & 70 & $<1$ & -- & -- & 4.2 & - & 650 & -- \\
\hline $09-25-84$ & 30 & -- & 30 & $<1$ & -- & -- & 1.2 & -- & 790 & -- \\
\hline $10-02-84$ & 32 & -- & 70 & $<1$ & -- & -- & 2.5 & -- & 570 & -- \\
\hline $10-09-84$ & 31 & -- & 60 & $<1$ & -- & -- & 1.2 & -- & 520 & -- \\
\hline $10-16-84$ & 33 & -- & 70 & $<1$ & -- & -- & 1.8 & -- & 670 & -- \\
\hline $10-23-84$ & 34 & -- & 130 & $<1$ & -- & -- & 2.2 & -- & 660 & -- \\
\hline $10-30-84$ & $\ldots$ & -- & -- & -- & -- & -- & -- & -- & -- & -- \\
\hline $11-06-84$ & -- & -- & 30 & $<1$ & -- & $-\infty$ & 3.6 & -- & 420 & -- \\
\hline $11-13-84$ & 34 & -- & 110 & $<1$ & -- & -- & 1.9 & -- & 270 & -- \\
\hline $11-20-84$ & 36 & 90 & 90 & $<1$ & $<1$ & $<1$ & 2.5 & 360 & 230 & 1 \\
\hline $11-27-84$ & 40 & -- & 80 & $<1$ & -- & -- & .2 & -- & 230 & -- \\
\hline $12-05-84$ & 35 & -- & 240 & $<1$ & -- & -- & 1.2 & -- & 210 & -- \\
\hline $12-11-84$ & 35 & -- & ${ }^{2} 70$ & $<1$ & -- & -- & .7 & -- & 260 & -- \\
\hline $12-19-84$ & 38 & $<10$ & $2<10$ & -- & -- & 4 & .4 & 430 & 260 & 4 \\
\hline $12-26-84$ & 34 & -- & ${ }^{2} 100$ & -- & -- & -- & .9 & -- & 170 & -- \\
\hline $01-02-85$ & 32 & -- & 280 & -- & -- & -- & .4 & -- & 180 & -- \\
\hline $01-08-85$ & 38 & -- & ${ }^{2} 80$ & -- & -- & -- & 1.6 & -- & 190 & -- \\
\hline $01-16-85$ & 44 & -- & ${ }^{2} 100$ & -- & - & -- & .3 & -- & 340 & -- \\
\hline $01-22-85$ & 57 & -- & ${ }^{2} 90$ & -- & -- & -- & 2.5 & -- & 410 & -- \\
\hline
\end{tabular}

see footnotes at end of table. 
Table 11.--Physical characteristics and concentrations of chemical constituents of streamflow at station number 01174050, East Branch Fever Brook near Petersham, Massachusetts--Continued

\begin{tabular}{|c|c|c|c|c|c|c|c|c|c|c|}
\hline Date & $\begin{array}{l}\text { Lead, } \\
\text { dis- } \\
\text { solved } \\
\text { ( } \mu \mathrm{g} / \mathrm{L} \\
\text { as } \mathrm{Pb})\end{array}$ & $\begin{array}{l}\text { Manga- } \\
\text { nese, } \\
\text { total } \\
\text { recov- } \\
\text { erable } \\
(\mu g / L \\
\text { as } M n)\end{array}$ & $\begin{array}{l}\text { Manga- } \\
\text { nese, } \\
\text { dis- } \\
\text { solved } \\
(\mu g / L \\
\text { as } M n)\end{array}$ & $\begin{array}{l}\text { Mercury, } \\
\text { total } \\
\text { recov- } \\
\text { erable } \\
\text { ( } \mu \mathrm{g} / \mathrm{L} \\
\text { as } \mathrm{Hg})\end{array}$ & $\begin{array}{l}\text { Mercury, } \\
\text { dis- } \\
\text { solved } \\
(\mu g / L \\
\text { as } \mathrm{Hg})\end{array}$ & $\begin{array}{c}\text { Sele- } \\
\text { nium, } \\
\text { total } \\
(\mu g / L \\
\text { as } \mathrm{Se})\end{array}$ & $\begin{array}{c}\text { Sele- } \\
\text { nium, } \\
\text { dis- } \\
\text { solved } \\
\text { ( } \mu \text { g/L } \\
\text { as Se) }\end{array}$ & $\begin{array}{c}\text { Vana- } \\
\text { dium, } \\
\text { dis- } \\
\text { solved } \\
(\mu g / L \\
\text { as V) }\end{array}$ & $\begin{array}{l}\text { Carbon, } \\
\text { organic } \\
\text { total } \\
(\mathrm{mg} / \mathrm{L} \\
\text { as C) }\end{array}$ & $\begin{array}{l}\text { Carbon, } \\
\text { organic, } \\
\text { dis- } \\
\text { solved } \\
\text { (mg/L } \\
\text { as C) }\end{array}$ \\
\hline $05-16-84$ & -- & -- & 28 & -- & -- & -- & -- & -- & -- & 4.5 \\
\hline $05-22-84$ & -- & -- & 24 & -- & $<0.1$ & -- & $<1$ & $<1$ & $=-$ & 4.7 \\
\hline $05-29-84$ & -- & -- & 40 & -- & -- & -- & $<1$ & $<1$ & -- & 6.2 \\
\hline $05-30-84$ & 1 & 40 & 40 & $<0.1$ & $<.1$ & $<1$ & $<1$ & $<1$ & 5.6 & 6.0 \\
\hline $05-30-84$ & -- & -- & ${ }^{2} 34$ & -- & -- & -- & -- & -- & -- & -- \\
\hline $05-31-84$ & $<0.1$ & 40 & 36 & $<.1$ & $<.1$ & $<1$ & $<1$ & 1 & 5.4 & -- \\
\hline $06-05-84$ & -- & -- & 33 & -- & -- & -- & -- & -- & -- & 5.1 \\
\hline $06-12-84$ & 1.3 & -- & 60 & -- & $<.1$ & -- & $<1$ & 1 & -- & 7.6 \\
\hline $06-19-84$ & -- & -- & 55 & -- & -- & -- & -- & -- & -- & 7.9 \\
\hline $06-26-84$ & .9 & -- & 59 & -- & .1 & -- & $<1$ & $<1$ & -- & 8.4 \\
\hline $07-03-84$ & -- & -- & 95 & -- & -- & -- & -- & -- & -- & 8.5 \\
\hline $07-10-84$ & .9 & -- & 51 & -- & $<.1$ & -- & $<1$ & $<1$ & -- & 8.6 \\
\hline $07-17-84$ & -- & -- & 66 & -- & -- & -- & -- & -- & -- & 8.8 \\
\hline $07-24-84$ & 1.5 & -- & 41 & -- & .3 & -- & $<1$ & 2 & -- & -- \\
\hline $07-31-84$ & 3.3 & -- & -- & -- & .4 & -- & $<1$ & 2 & -- & 9.7 \\
\hline $08-07-84$ & 1.9 & -- & -- & -- & .3 & -- & $<1$ & 2 & -- & -- \\
\hline $08-14-84$ & 2.1 & -- & -- & -- & $<.1$ & -- & $<1$ & 2 & -- & 10 \\
\hline $08-21-84$ & 1.3 & -- & -- & -- & $<.1$ & -- & $<1$ & 1 & -- & 9.6 \\
\hline $08-28-84$ & 1.2 & -- & -- & -- & $<.1$ & -- & $<1$ & 1 & -- & 8.8 \\
\hline $09-04-84$ & 2.2 & -- & -- & -- & $<.1$ & -- & $<1$ & 1 & -- & -- \\
\hline $09-11-84$ & 1.3 & 90 & 68 & .3 & .2 & $<1$ & $<1$ & $<1$ & -- & -- \\
\hline $09-18-84$ & 1.8 & -- & 56 & -- & $<.1$ & -- & $<1$ & $<1$ & -- & 10 \\
\hline $09-25-84$ & 1.3 & -- & 100 & -- & $<.1$ & -- & $<1$ & 1 & -- & 9.5 \\
\hline $10-02-84$ & 1.4 & -- & 85 & -- & $<.1$ & -- & $<1$ & $<1$ & -- & 5.3 \\
\hline $10-09-84$ & 1.1 & -- & 47 & -- & $<.1$ & -- & $<1$ & 1 & -- & -- \\
\hline $10-16-84$ & 1.3 & -- & 77 & -- & $<.1$ & -- & $<1$ & 1 & -- & 11 \\
\hline $10-23-84$ & 1.9 & -- & 80 & -- & .2 & -- & $<1$ & 1 & -- & 12 \\
\hline $10-30-84$ & -- & -- & -- & -- & -- & -- & -- & -- & -- & -- \\
\hline $11-06-84$ & 1.5 & -- & 62 & -- & $<.1$ & -- & $<1$ & 1 & -- & 9.4 \\
\hline $11-13-84$ & .6 & -- & 45 & -- & $<.1$ & -- & $<1$ & $<1$ & -- & 8.8 \\
\hline $11-20-84$ & 1.1 & 40 & 38 & $<.1$ & -- & $<1$ & $<1$ & 1 & -- & 10 \\
\hline $11-27-84$ & .3 & -- & 37 & -- & -- & -- & $<1$ & $<1$ & -- & 8.8 \\
\hline $12-05-84$ & .4 & -- & 28 & -- & -- & -- & $<1$ & $<1$ & 7.6 & -- \\
\hline $12-11-84$ & .3 & -- & 33 & -- & .3 & -- & $<1$ & $<1$ & -- & -- \\
\hline $12-19-84$ & .3 & 40 & 33 & -- & -- & -- & -- & -- & -- & 5.8 \\
\hline $12-26-84$ & .2 & -- & -- & -- & -- & -- & -- & -- & -- & -- \\
\hline $01-02-85$ & .4 & -- & 26 & -- & -- & -- & -- & -- & -- & -- \\
\hline $01-08-85$ & .8 & -- & 27 & -- & -- & -- & -- & -- & -- & 6.0 \\
\hline $01-16-85$ & .2 & -- & 35 & -- & -- & -- & -- & -- & -- & 5.8 \\
\hline $01-22-85$ & .1 & -- & 37 & -- & -- & -- & -- & -- & -- & 5.6 \\
\hline
\end{tabular}

see footnotes at end of table. 
Table 11.--Physical characteristics and concentrations of chemical constituents of streamflow at station number 01174050, East Branch Fever Brook near Petersham, Massachusetts--Continued

\begin{tabular}{|c|c|c|c|c|c|c|c|c|c|}
\hline Date & Time & $\begin{array}{l}\text { Stream- } \\
\text { flow, } \\
\text { instan- } \\
\text { taneous } \\
\left(\mathrm{ft}^{3} / \mathrm{s}\right)\end{array}$ & $\begin{array}{l}\text { Spe- } \\
\text { cific } \\
\text { con- } \\
\text { duct- } \\
\text { ance } \\
(\mu \mathrm{s} / \mathrm{cm})\end{array}$ & $\begin{array}{c}\mathrm{pH} \\
\text { (stand- } \\
\text { ard } \\
\text { units) }\end{array}$ & $\begin{array}{l}\text { Temper- } \\
\text { ature } \\
\left({ }^{\circ} \mathrm{C}\right)\end{array}$ & $\begin{array}{l}\text { Hard- } \\
\text { ness } \\
(\mathrm{mg} / \mathrm{L} \\
\mathrm{as} \\
\left.\mathrm{CaCO}_{3}\right)\end{array}$ & $\begin{array}{l}\text { Calcium, } \\
\text { dis- } \\
\text { solved } \\
\text { (mg/L } \\
\text { as Ca) }\end{array}$ & $\begin{array}{l}\text { Magne- } \\
\text { sium, } \\
\text { dis- } \\
\text { solved } \\
\text { (mg/L } \\
\text { as Mg) }\end{array}$ & $\begin{array}{l}\text { Sodium, } \\
\text { dis- } \\
\text { solved } \\
\text { (mg/I } \\
\text { as } \mathrm{Na} \text { ) }\end{array}$ \\
\hline $\begin{array}{l}01-29-85^{1} \\
02-05-85 \\
02-13-85 \\
02-19-85^{1} \\
02-25-85\end{array}$ & $\begin{array}{l}09: 30 \\
09: 30 \\
16: 45 \\
09: 30 \\
15: 45\end{array}$ & $\begin{array}{l}0.96 \\
1.3 \\
11 \\
3.9 \\
13\end{array}$ & $\begin{array}{l}70 \\
69 \\
61 \\
69 \\
53\end{array}$ & $\begin{array}{l}6.0 \\
6.1 \\
6.0 \\
6.1 \\
5.8\end{array}$ & $\begin{array}{r}0.5 \\
.5 \\
.5 \\
2.0 \\
3.5\end{array}$ & $\begin{array}{l}12 \\
13 \\
11 \\
11 \\
10\end{array}$ & $\begin{array}{l}3.1 \\
3.4 \\
2.9 \\
3.0 \\
2.5\end{array}$ & $\begin{array}{l}1.0 \\
1.0 \\
.88 \\
.96 \\
.85\end{array}$ & $\begin{array}{l}6.8 \\
6.9 \\
5.7 \\
7.1 \\
5.0\end{array}$ \\
\hline $\begin{array}{l}03-05-85 \\
03-12-85 \\
03-19-85 \\
03-26-85 \\
04-02-85\end{array}$ & $\begin{array}{l}13: 30 \\
11: 30 \\
14: 30 \\
09: 00 \\
09: 45\end{array}$ & $\begin{array}{l}7.1 \\
29 \\
11 \\
6.0 \\
6.7\end{array}$ & $\begin{array}{l}57 \\
-- \\
54 \\
50 \\
52\end{array}$ & $\begin{array}{l}6.0 \\
5.9 \\
5.7 \\
6.0 \\
6.0\end{array}$ & $\begin{array}{l}2.5 \\
3.0 \\
3.0 \\
2.5 \\
5.5\end{array}$ & $\begin{array}{r}10 \\
8 \\
8 \\
-- \\
9\end{array}$ & $\begin{array}{r}2.5 \\
2.1 \\
2.1 \\
-- \\
2.3\end{array}$ & $\begin{array}{r}.81 \\
.61 \\
.78 \\
-\quad \\
.76\end{array}$ & $\begin{array}{r}5.9 \\
4.1 \\
5.3 \\
-- \\
5.4\end{array}$ \\
\hline $\begin{array}{l}04-09-85 \\
04-16-85 \\
04-23-851 \\
04-30-85 \\
05-07-85\end{array}$ & $\begin{array}{l}10: 00 \\
09: 30 \\
10: 15 \\
09: 30 \\
09: 15\end{array}$ & $\begin{array}{r}11 \\
5.7 \\
1.9 \\
2.2 \\
4.6\end{array}$ & $\begin{array}{l}55 \\
57 \\
59 \\
59 \\
57\end{array}$ & $\begin{array}{l}6.0 \\
5.9 \\
6.0 \\
6.2 \\
6.1\end{array}$ & $\begin{array}{l}8.0 \\
12.0 \\
15.0 \\
14.0 \\
11.5\end{array}$ & $\begin{array}{r}9 \\
9 \\
9 \\
10 \\
7\end{array}$ & $\begin{array}{l}2.4 \\
2.4 \\
2.4 \\
2.5 \\
1.7\end{array}$ & $\begin{array}{l}.75 \\
.77 \\
.84 \\
.80 \\
.59\end{array}$ & $\begin{array}{l}5.9 \\
6.4 \\
6.6 \\
6.2 \\
4.2\end{array}$ \\
\hline $\begin{array}{l}05-14-85^{1} \\
05-21-85 \\
05-28-85 \\
06-04-85 \\
06-11-85\end{array}$ & $\begin{array}{l}09: 30 \\
08: 30 \\
08: 00 \\
09: 00 \\
09: 15\end{array}$ & $\begin{array}{l}1.3 \\
1.8 \\
2.9 \\
6.6 \\
2.4\end{array}$ & $\begin{array}{l}60 \\
58 \\
50 \\
56 \\
58\end{array}$ & $\begin{array}{l}6.1 \\
6.0 \\
5.7 \\
5.7 \\
5.6\end{array}$ & $\begin{array}{l}19.5 \\
17.0 \\
19.0 \\
18.0 \\
18.5\end{array}$ & $\begin{array}{r}9 \\
10 \\
-- \\
9 \\
--\end{array}$ & $\begin{array}{l}2.5 \\
2.5 \\
-- \\
--\end{array}$ & $\begin{array}{r}.75 \\
-.87 \\
-\quad .75 \\
-\end{array}$ & $\begin{array}{l}6.7 \\
6.7 \\
-- \\
6.5 \\
--\end{array}$ \\
\hline $\begin{array}{l}06-18-85 \\
06-25-85 \\
07-02-85 \\
07-10-85 \\
07-16-85\end{array}$ & $\begin{array}{l}09: 30 \\
16: 30 \\
09: 00 \\
14: 15 \\
09: 30\end{array}$ & $\begin{array}{r}5.1 \\
.9 \\
2.4 \\
.8 \\
.95\end{array}$ & $\begin{array}{l}54 \\
58 \\
57 \\
65 \\
58\end{array}$ & $\begin{array}{l}5.8 \\
5.5 \\
5.7 \\
5.7 \\
5.7\end{array}$ & $\begin{array}{l}17.0 \\
19.0 \\
18.5 \\
25.0 \\
21.5\end{array}$ & $\begin{array}{r}8 \\
-- \\
-9 \\
--\end{array}$ & $\begin{array}{c}2.1 \\
-- \\
-2.3 \\
-2.5\end{array}$ & $\begin{array}{r}-.68 \\
-.73 \\
.81\end{array}$ & $\begin{array}{c}6.6 \\
-- \\
-6.9 \\
-6.4\end{array}$ \\
\hline $\begin{array}{l}07-23-85^{1} \\
07-30-85^{1} \\
08-08-85 \\
08-13-85 \\
08-20-85\end{array}$ & $\begin{array}{l}10: 10 \\
09: 15 \\
09: 00 \\
09: 15 \\
19: 30\end{array}$ & $\begin{array}{l}.48 \\
.53 \\
1.4 \\
.62 \\
4.2\end{array}$ & $\begin{array}{l}56 \\
54 \\
56 \\
63 \\
64\end{array}$ & $\begin{array}{l}5.8 \\
5.9 \\
5.6 \\
5.7 \\
5.6\end{array}$ & $\begin{array}{l}19.5 \\
20.5 \\
19.5 \\
18.5 \\
19.5\end{array}$ & $\begin{array}{l}-- \\
10 \\
-- \\
10 \\
--\end{array}$ & $\begin{array}{l}-- \\
-2.6 \\
-- \\
--\end{array}$ & --.78 & $\begin{array}{c}-- \\
6.1 \\
-- \\
6.2 \\
--\end{array}$ \\
\hline $\begin{array}{l}08-27-85 \\
09-04-85 \\
09-10-85\end{array}$ & $\begin{array}{l}09: 00 \\
09: 00 \\
09: 30\end{array}$ & $\begin{array}{l}7.0 \\
5.5 \\
4.0\end{array}$ & $\begin{array}{l}65 \\
68 \\
57\end{array}$ & $\begin{array}{l}5.7 \\
5.6 \\
5.7\end{array}$ & $\begin{array}{l}19.0 \\
19.5 \\
17.5\end{array}$ & $\begin{array}{l}10 \\
-- \\
--\end{array}$ & $\begin{array}{l}2.5 \\
-- \\
--\end{array}$ & $--^{.84}$ & $\begin{array}{l}6.5 \\
-- \\
--\end{array}$ \\
\hline
\end{tabular}


Table 11.--Physical characteristics and concentrations of chemical constituents of streamflow at station number 01174050, East Branch Fever Brook near Petersham, Massachusetts--Continued

\begin{tabular}{|c|c|c|c|c|c|c|c|c|c|c|c|}
\hline Date & $\begin{array}{l}\text { Potas- } \\
\text { sium, } \\
\text { dis- } \\
\text { solved } \\
\text { (mg/L } \\
\text { as K) }\end{array}$ & $\begin{array}{c}\text { Nitro- } \\
\text { gen, } \\
\text { ammonia } \\
\text { total } \\
\text { (mg/L } \\
\text { as } N \text { ) }\end{array}$ & $\begin{array}{c}\text { Nitro- } \\
\text { gen, } \\
\text { ammonia, } \\
\text { dis- } \\
\text { solved } \\
\text { (mg/L } \\
\text { as N) }\end{array}$ & $\begin{array}{c}\text { Alka- } \\
\text { linity } \\
\text { field } \\
\text { (mg/L } \\
\text { as } \\
\mathrm{CaCO}_{3} \text { ) }\end{array}$ & $\begin{array}{l}\text { Sulfate, } \\
\text { dis- } \\
\text { solved } \\
\left(\mathrm{mg}^{\prime} \mathrm{L}\right. \\
\left.\text { as } \mathrm{SO}_{4}\right)\end{array}$ & $\begin{array}{c}\text { Nitro- } \\
\text { gen, } \\
\text { nitrate, } \\
\text { dis- } \\
\text { solved } \\
\text { (mg/L } \\
\text { as N) }\end{array}$ & $\begin{array}{l}\text { Phos- } \\
\text { phorus, } \\
\text { ortho, } \\
\text { dis- } \\
\text { solved } \\
\text { (mg/L } \\
\text { as P) }\end{array}$ & $\begin{array}{c}\text { Phos- } \\
\text { phorus, } \\
\text { dis- } \\
\text { solved } \\
\text { (mg/L } \\
\text { as P) }\end{array}$ & $\begin{array}{l}\text { Chlo- } \\
\text { ride, } \\
\text { dis- } \\
\text { solved } \\
\text { (mg/L } \\
\text { as Cl) }\end{array}$ & $\begin{array}{l}\text { Fluo- } \\
\text { ride, } \\
\text { dis- } \\
\text { solved } \\
\text { (mg/L } \\
\text { as F) }\end{array}$ & $\begin{array}{l}\text { Silica, } \\
\text { dis- } \\
\text { solved } \\
\text { (mg/L } \\
\text { as } \\
\mathrm{SiO}_{2} \text { ) }\end{array}$ \\
\hline $\begin{array}{l}01-29-85 \\
02-05-85 \\
02-13-85 \\
02-19-85 \\
02-25-85\end{array}$ & $\begin{array}{r}1.0 \\
.91 \\
.95 \\
.90 \\
.77\end{array}$ & $\begin{array}{l}-- \\
-- \\
-- \\
-- \\
--\end{array}$ & $\begin{array}{r}0.16 \\
.16 \\
.14 \\
.08 \\
.09\end{array}$ & $\begin{array}{l}-- \\
3.3 \\
3.1 \\
2.0 \\
1.4\end{array}$ & $\begin{array}{l}7.7 \\
8.4 \\
7.5 \\
8.2 \\
3.5\end{array}$ & $\begin{array}{r}0.03 \\
.09 \\
.15 \\
.11 \\
<.01\end{array}$ & $\begin{array}{r}<0.01 \\
<.01 \\
<.01 \\
<.01 \\
<.01\end{array}$ & $\begin{array}{l}-- \\
-- \\
-- \\
-- \\
--\end{array}$ & $\begin{array}{l}11 \\
12 \\
9.8 \\
3<.01 \\
6.6\end{array}$ & $\begin{array}{l}0.04 \\
<.01 \\
<.01 \\
.10 \\
.13\end{array}$ & $\begin{array}{l}8.2 \\
8.5 \\
7.7 \\
7.7 \\
6.3\end{array}$ \\
\hline $\begin{array}{l}03-05-85 \\
03-12-85 \\
03-19-85 \\
03-26-85 \\
04-02-85\end{array}$ & $\begin{array}{r}.70 \\
.66 \\
.64 \\
-\quad \\
-65\end{array}$ & $\begin{array}{l}-- \\
-- \\
-- \\
-- \\
--\end{array}$ & $\begin{array}{r}<.01 \\
.03 \\
<.01 \\
<.01 \\
.22\end{array}$ & $\begin{array}{r}1.2 \\
.8 \\
.8 \\
1.0 \\
1.2\end{array}$ & $\begin{array}{r}6.5 \\
7.5 \\
6.0 \\
-\overline{3} .2\end{array}$ & $\begin{array}{r}.43 \\
.04 \\
-.51 \\
-- \\
<.01\end{array}$ & $\begin{array}{l}<.01 \\
<.01 \\
<.01 \\
-- \\
<.01\end{array}$ & $\begin{array}{l}-- \\
-- \\
-- \\
-- \\
--\end{array}$ & $\begin{array}{c}6.3 \\
6.0 \\
7.8 \\
-\frac{3}{3} .05\end{array}$ & $\begin{array}{r}.10 \\
.06 \\
.16 \\
-.03\end{array}$ & $\begin{array}{l}5.1 \\
4.3 \\
4.3 \\
-- \\
2.9\end{array}$ \\
\hline $\begin{array}{l}04-09-85 \\
04-16-85 \\
04-23-85 \\
04-30-85 \\
05-07-85\end{array}$ & $\begin{array}{l}.68 \\
.68 \\
.73 \\
.76 \\
.25\end{array}$ & $\begin{array}{l}-- \\
-- \\
-- \\
-- \\
--\end{array}$ & $\begin{array}{r}.12 \\
.02 \\
.03 \\
.05 \\
<.01\end{array}$ & $\begin{array}{l}1.0 \\
1.4 \\
2.1 \\
2.3 \\
2.3\end{array}$ & $\begin{array}{r}6.4 \\
5.9 \\
5.7 \\
5.1 \\
32.7\end{array}$ & $\begin{array}{r}.02 \\
<.01 \\
<.01 \\
<.01 \\
.02\end{array}$ & $\begin{array}{l}<.01 \\
<.01 \\
<.01 \\
<.01 \\
<.01\end{array}$ & $\begin{array}{l}-- \\
-- \\
-- \\
-- \\
--\end{array}$ & $\begin{array}{r}9.5 \\
9.5 \\
9.9 \\
\frac{1}{3} 0 \\
4.0\end{array}$ & $\begin{array}{l}.07 \\
.32 \\
.06 \\
.07 \\
.04\end{array}$ & $\begin{array}{l}3.0 \\
2.3 \\
1.7 \\
1.3 \\
1.3\end{array}$ \\
\hline $\begin{array}{l}05-14-85 \\
05-21-85 \\
05-28-85 \\
06-04-85 \\
06-11-85\end{array}$ & $\begin{array}{r}.72 \\
-.61 \\
-. .51\end{array}$ & $\begin{array}{l}-- \\
-- \\
-- \\
--\end{array}$ & $\begin{array}{r}.05 \\
-.04 \\
-. \\
-.04\end{array}$ & $\begin{array}{l}2.1 \\
2.1 \\
1.6 \\
1.2 \\
1.2\end{array}$ & $\begin{array}{c}5.5 \\
5.6 \\
-- \\
7.4 \\
--\end{array}$ & $\begin{array}{c}<.01 \\
<.01 \\
-- \\
{ }_{--}\end{array}$ & $\begin{array}{l}<.01 \\
<.01 \\
-- \\
<.01 \\
--\end{array}$ & $\begin{array}{l}-- \\
-- \\
-- \\
-- \\
--\end{array}$ & $\begin{array}{l}11 \\
11 \\
-- \\
9.7 \\
--\end{array}$ & $\begin{array}{r}.09 \\
-.10 \\
-.12\end{array}$ & $\begin{array}{l}1.2 \\
1.3 \\
-- \\
3.2 \\
--\end{array}$ \\
\hline $\begin{array}{l}06-18-85 \\
06-25-85 \\
07-02-85 \\
07-10-85 \\
07-16-85\end{array}$ & $\begin{array}{r}-.48 \\
-.61 \\
-.46\end{array}$ & $\begin{array}{l}-- \\
-- \\
-- \\
--\end{array}$ & $\begin{array}{r}<.01 \\
-- \\
-.09 \\
-.05\end{array}$ & $\begin{array}{l}1.4 \\
1.8 \\
1.9 \\
2.7 \\
2.3\end{array}$ & $\begin{array}{c}5.3 \\
-- \\
6.0 \\
-- \\
3.4\end{array}$ & $\begin{array}{r}-.07 \\
-- \\
-.01 \\
.02\end{array}$ & $\begin{array}{l}<.01 \\
-- \\
<.01 \\
-- \\
<.01\end{array}$ & $\begin{array}{l}-- \\
-- \\
-- \\
-- \\
--\end{array}$ & $\begin{array}{l}\frac{10}{3} .1 \\
-- \\
11\end{array}$ & --.06 & $\begin{array}{l}2.9 \\
-- \\
3.7 \\
-- \\
3.7\end{array}$ \\
\hline $\begin{array}{l}07-23-85 \\
07-30-85 \\
08-08-85 \\
08-13-85 \\
08-20-85\end{array}$ & ${ }_{-.}^{-.39}$ & $\begin{array}{l}-- \\
-- \\
-- \\
-- \\
--\end{array}$ & ${ }_{--}^{-} .07$ & $\begin{array}{l}2.3 \\
3.2 \\
2.1 \\
3.4 \\
2.7\end{array}$ & $\begin{array}{c}-- \\
-3.4 \\
-- \\
--\end{array}$ & ${ }_{--}^{-} .02$ & $\begin{array}{l}-- \\
<.01 \\
-- \\
<.01 \\
--\end{array}$ & $\begin{array}{l}-- \\
-- \\
-- \\
-- \\
--\end{array}$ & $\begin{array}{l}-- \\
-- \\
-- \\
10 \\
--\end{array}$ & --.09 & $\begin{array}{l}-- \\
4.9 \\
-- \\
4.6 \\
--\end{array}$ \\
\hline $\begin{array}{l}08-27-85 \\
09-04-85 \\
09-10-85\end{array}$ & $-_{--}^{.56}$ & $\begin{array}{l}-- \\
-- \\
--\end{array}$ & $\begin{array}{l}<.01 \\
-- \\
--\end{array}$ & $\begin{array}{c}2.5 \\
-- \\
2.8\end{array}$ & $\begin{array}{l}4.5 \\
-- \\
--\end{array}$ & ${ }_{--}^{.01}$ & $\begin{array}{l}<.01 \\
-- \\
--\end{array}$ & $\begin{array}{l}-- \\
-- \\
--\end{array}$ & $\begin{array}{l}10 \\
-- \\
--\end{array}$ & $-{ }_{--}^{.09}$ & $\begin{array}{l}3.9 \\
-- \\
--\end{array}$ \\
\hline
\end{tabular}

See footnotes at end of table. 
Table 11.--Physical characteristics and concentrations of chemical constituents of streamflow at station number 01174050, East Branch Fever Brook near Petersham, Massachusetts--Continued

\begin{tabular}{|c|c|c|c|c|c|c|c|c|c|c|}
\hline Date & $\begin{array}{l}\text { Solids, } \\
\text { sum of } \\
\text { consti- } \\
\text { tuents, } \\
\text { dis- } \\
\text { solved } \\
\text { (mg/L) }\end{array}$ & $\begin{array}{l}\text { Alum- } \\
\text { inum, } \\
\text { total } \\
\text { recov- } \\
\text { erable } \\
(\mu g / L \\
\text { as Al) }\end{array}$ & $\begin{array}{l}\text { Alum- } \\
\text { inum, } \\
\text { dis- } \\
\text { solved } \\
(\mu g / L \\
\text { as Al) }\end{array}$ & $\begin{array}{c}\text { Arsenic, } \\
\text { dis- } \\
\text { solved } \\
\text { ( } \mu g / L \\
\text { as As) }\end{array}$ & $\begin{array}{c}\text { Arsenic, } \\
\text { total } \\
(\mu g / L \\
\text { as As) }\end{array}$ & $\begin{array}{c}\text { Copper } \\
\text { total } \\
\text { recov- } \\
\text { erable } \\
\text { ( } \mu \text { g/L } \\
\text { as Cu) }\end{array}$ & $\begin{array}{c}\text { Copper, } \\
\text { dis- } \\
\text { solved } \\
\text { ( } \mu g / L \\
\text { as Cu) }\end{array}$ & $\begin{array}{l}\text { Iron, } \\
\text { total } \\
\text { recov- } \\
\text { erable } \\
(\mu g / L \\
\text { as Fe) }\end{array}$ & $\begin{array}{l}\text { Iron, } \\
\text { dis- } \\
\text { solved } \\
(\mu g / L \\
\text { as Fe) }\end{array}$ & $\begin{array}{l}\text { Lead, } \\
\text { total } \\
\text { recov- } \\
\text { erable } \\
(\mu g / L \\
\text { as Pb) }\end{array}$ \\
\hline $\begin{array}{l}01-29-85 \\
02-05-85 \\
02-13-85 \\
02-19-85 \\
02-25-85\end{array}$ & $\begin{array}{l}-- \\
45 \\
39 \\
-- \\
24\end{array}$ & $\begin{array}{l}-- \\
-- \\
-- \\
-- \\
--\end{array}$ & $\begin{array}{r}{ }_{2} 220 \\
2_{220} 20 \\
270 \\
280 \\
2_{100}\end{array}$ & $\begin{array}{l}-- \\
-- \\
-- \\
-- \\
--\end{array}$ & $\begin{array}{l}-- \\
-- \\
-- \\
-- \\
--\end{array}$ & $\begin{array}{l}-- \\
-- \\
-- \\
-- \\
--\end{array}$ & $\begin{array}{l}0.6 \\
5.0 \\
2.1 \\
1.6 \\
1.4\end{array}$ & $\begin{array}{l}-- \\
-- \\
-- \\
-- \\
--\end{array}$ & $\begin{array}{l}430 \\
550 \\
470 \\
290 \\
190\end{array}$ & $\begin{array}{l}-- \\
-- \\
-- \\
-- \\
--\end{array}$ \\
\hline $\begin{array}{l}03-05-85 \\
03-12-85 \\
03-19-85 \\
03-26-85 \\
04-02-85\end{array}$ & $\begin{array}{l}31 \\
26 \\
30 \\
-- \\
14\end{array}$ & $\begin{array}{l}-- \\
-- \\
-- \\
-- \\
--\end{array}$ & $\begin{array}{r}280 \\
270 \\
2300 \\
280 \\
2_{80}\end{array}$ & $\begin{array}{l}-- \\
-- \\
-- \\
-- \\
--\end{array}$ & $\begin{array}{l}-- \\
-- \\
-- \\
-- \\
--\end{array}$ & $\begin{array}{l}-- \\
-- \\
-- \\
--\end{array}$ & $\begin{array}{r}1.7 \\
1.1 \\
1.3 \\
.5 \\
.6\end{array}$ & $\begin{array}{l}-- \\
-- \\
-- \\
--\end{array}$ & $\begin{array}{r}110 \\
100 \\
76 \\
-- \\
130\end{array}$ & $\begin{array}{l}-- \\
-- \\
-- \\
-- \\
--\end{array}$ \\
\hline $\begin{array}{l}04-09-85 \\
04-16-85 \\
04-23-85 \\
04-30-85 \\
05-07-85\end{array}$ & $\begin{array}{l}30 \\
29 \\
30 \\
29 \\
17\end{array}$ & $\begin{array}{l}-- \\
-- \\
-- \\
-- \\
--\end{array}$ & $\begin{array}{r}270 \\
260 \\
270 \\
2790 \\
2_{100}\end{array}$ & $\begin{array}{l}-- \\
-- \\
-- \\
-- \\
--\end{array}$ & $\begin{array}{l}-- \\
-- \\
-- \\
-- \\
--\end{array}$ & $\begin{array}{l}-- \\
-- \\
-- \\
-- \\
--\end{array}$ & $\begin{array}{r}1.7 \\
.8 \\
.1 \\
<.1 \\
2.0\end{array}$ & $\begin{array}{l}-- \\
-- \\
-- \\
-- \\
--\end{array}$ & $\begin{array}{l}110 \\
120 \\
190 \\
280 \\
240\end{array}$ & $\begin{array}{l}-- \\
-- \\
-- \\
-- \\
--\end{array}$ \\
\hline $\begin{array}{l}05-14-85 \\
05-21-85 \\
05-28-85 \\
06-04-85 \\
06-11-85\end{array}$ & $\begin{array}{l}30 \\
30 \\
-- \\
32 \\
--\end{array}$ & $\begin{array}{l}-- \\
-- \\
-- \\
-- \\
--\end{array}$ & $\begin{array}{r}280 \\
280 \\
2-- \\
2<10 \\
--\end{array}$ & $\begin{array}{l}-- \\
-- \\
-- \\
-- \\
--\end{array}$ & $\begin{array}{l}-- \\
-- \\
-- \\
-- \\
--\end{array}$ & $\begin{array}{l}-- \\
-- \\
-- \\
-- \\
--\end{array}$ & $\begin{array}{l}<.1 \\
1.0 \\
-- \\
3.0 \\
--\end{array}$ & $\begin{array}{l}-- \\
-- \\
-- \\
--\end{array}$ & $\begin{array}{r}250 \\
300 \\
-- \\
310 \\
--\end{array}$ & $\begin{array}{l}-- \\
-- \\
-- \\
-- \\
--\end{array}$ \\
\hline $\begin{array}{l}06-18-85 \\
06-25-85 \\
07-02-85 \\
07-10-85 \\
07-16-85\end{array}$ & $\begin{array}{l}30 \\
-- \\
22 \\
-- \\
31\end{array}$ & $\begin{array}{l}-- \\
-- \\
-- \\
-- \\
--\end{array}$ & $\begin{array}{r}270 \\
2<70 \\
=- \\
260\end{array}$ & $\begin{array}{l}-- \\
-- \\
-- \\
-- \\
--\end{array}$ & $\begin{array}{l}-- \\
-- \\
-- \\
-- \\
--\end{array}$ & $\begin{array}{l}-- \\
-- \\
-- \\
-- \\
--\end{array}$ & $\begin{array}{l}<.1 \\
-- \\
<.1 \\
-- \\
<.1\end{array}$ & $\begin{array}{l}-- \\
-- \\
-- \\
-- \\
--\end{array}$ & $\begin{array}{r}390 \\
-- \\
580 \\
-- \\
600\end{array}$ & $\begin{array}{l}-- \\
-- \\
-- \\
--\end{array}$ \\
\hline $\begin{array}{l}07-23-85 \\
07-30-85\end{array}$ & -- & -- & $2--$ & -- & -- & -- & $--\overline{2}$ & -- & $6 \overline{0}$ & -- \\
\hline $\begin{array}{l}08-08-85 \\
08-13-85\end{array}$ & $\overline{32}$ & -- & $2--$ & -- & -- & $\begin{array}{l}-- \\
--\end{array}$ & $\begin{array}{l}-- \\
<.1\end{array}$ & -- & 730 & $\begin{array}{l}-- \\
--\end{array}$ \\
\hline $08-20-85$ & -- & -- & -- & -- & -- & -- & -- & -- & -- & -- \\
\hline $\begin{array}{l}08-27-85 \\
09-04-85 \\
09-10-85\end{array}$ & $\begin{array}{l}31 \\
-- \\
--\end{array}$ & $\begin{array}{l}-- \\
-- \\
--\end{array}$ & $\begin{array}{l}280 \\
-- \\
--\end{array}$ & $\begin{array}{l}-- \\
-- \\
--\end{array}$ & $\begin{array}{l}-- \\
-- \\
--\end{array}$ & $\begin{array}{l}-- \\
-- \\
--\end{array}$ & $\begin{array}{l}3.0 \\
-- \\
--\end{array}$ & $\begin{array}{l}-- \\
--\end{array}$ & $\begin{array}{r}480 \\
-- \\
--\end{array}$ & $\begin{array}{l}-- \\
-- \\
--\end{array}$ \\
\hline
\end{tabular}


Table 11.--Physical characteristics and concentrations of chemical constituents of streamflow at station number 01174050, East Branch Fever Brook near Petersham, Massachusetts--Continued

\begin{tabular}{|c|c|c|c|c|c|c|c|c|c|c|}
\hline Date & $\begin{array}{l}\text { Lead, } \\
\text { dis- } \\
\text { solved } \\
(\mu \mathrm{g} / \mathrm{L} \\
\text { as } \mathrm{Pb})\end{array}$ & $\begin{array}{l}\text { Manga- } \\
\text { nese, } \\
\text { total } \\
\text { recov- } \\
\text { erable } \\
(\mu g / L \\
\text { as } M n)\end{array}$ & $\begin{array}{l}\text { Manga- } \\
\text { nese, } \\
\text { dis- } \\
\text { solved } \\
(\mu g / L \\
\text { as } M n)\end{array}$ & $\begin{array}{c}\text { Mercury, } \\
\text { total } \\
\text { recov- } \\
\text { erable } \\
(\mu g / L \\
\text { as } \mathrm{Hg})\end{array}$ & $\begin{array}{l}\text { Mercury, } \\
\text { dis- } \\
\text { solved } \\
\text { ( } \mu \text { g/L } \\
\text { as Hg) }\end{array}$ & $\begin{array}{l}\text { Sele- } \\
\text { nium, } \\
\text { total } \\
(\mu \mathrm{g} / \mathrm{L} \\
\text { as Se) }\end{array}$ & $\begin{array}{l}\text { Sele- } \\
\text { nium, } \\
\text { dis- } \\
\text { solved } \\
(\mu g / L \\
\text { as Se) }\end{array}$ & $\begin{array}{l}\text { Vana- } \\
\text { dium, } \\
\text { dis- } \\
\text { solved } \\
(\mu g / L \\
\text { as V) }\end{array}$ & $\begin{array}{c}\text { Carbon, } \\
\text { organic } \\
\text { total } \\
\text { (mg/L } \\
\text { as C) }\end{array}$ & $\begin{array}{l}\text { Carbon, } \\
\text { organic, } \\
\text { dis- } \\
\text { solved } \\
\text { (mg } / L \\
\text { as C) }\end{array}$ \\
\hline $\begin{array}{l}01-29-85 \\
02-05-85 \\
02-13-85 \\
02-19-85 \\
02-25-85\end{array}$ & $\begin{array}{l}0.1 \\
.1 \\
.7 \\
.3 \\
.1\end{array}$ & $\begin{array}{l}-- \\
-- \\
-- \\
-- \\
--\end{array}$ & $\begin{array}{l}36 \\
39 \\
64 \\
52 \\
56\end{array}$ & $\begin{array}{l}-- \\
-- \\
-- \\
--\end{array}$ & $\begin{array}{l}-- \\
-- \\
-- \\
-- \\
--\end{array}$ & $\begin{array}{l}-- \\
-- \\
-- \\
-- \\
--\end{array}$ & $\begin{array}{l}-- \\
-- \\
-- \\
--\end{array}$ & $\begin{array}{l}-- \\
-- \\
-- \\
-- \\
--\end{array}$ & $\begin{array}{l}-- \\
-- \\
-- \\
--\end{array}$ & $\begin{array}{l}5.6 \\
5.8 \\
5.2 \\
5.3 \\
5.5\end{array}$ \\
\hline $\begin{array}{l}03-05-85 \\
03-12-85 \\
03-19-85 \\
03-26-85 \\
04-02-85\end{array}$ & $\begin{array}{r}1.0 \\
.8 \\
.6 \\
.7 \\
1.0\end{array}$ & $\begin{array}{l}-- \\
-- \\
-- \\
-- \\
--\end{array}$ & $\begin{array}{l}28 \\
28 \\
31 \\
-- \\
31\end{array}$ & $\begin{array}{l}-- \\
-- \\
-- \\
-- \\
--\end{array}$ & $\begin{array}{l}-- \\
-- \\
-- \\
-- \\
--\end{array}$ & $\begin{array}{l}-- \\
-- \\
-- \\
-- \\
--\end{array}$ & $\begin{array}{l}-- \\
-- \\
-- \\
-- \\
--\end{array}$ & $\begin{array}{l}-- \\
-- \\
-- \\
-- \\
--\end{array}$ & $\begin{array}{l}-- \\
-- \\
-- \\
-- \\
--\end{array}$ & $\begin{array}{r}4.3 \\
-- \\
4.5 \\
4.6 \\
5.1\end{array}$ \\
\hline $\begin{array}{l}04-09-85 \\
04-16-85 \\
04-23-85 \\
04-30-85 \\
05-07-85\end{array}$ & $\begin{array}{r}1.4 \\
.4 \\
.3 \\
1.0 \\
--\end{array}$ & $\begin{array}{l}-- \\
-- \\
-- \\
-- \\
--\end{array}$ & $\begin{array}{l}29 \\
29 \\
38 \\
37 \\
26\end{array}$ & $\begin{array}{l}-- \\
-- \\
-- \\
--\end{array}$ & $\begin{array}{l}-- \\
-- \\
-- \\
-- \\
--\end{array}$ & $\begin{array}{l}-- \\
-- \\
-- \\
-- \\
--\end{array}$ & $\begin{array}{l}-- \\
-- \\
-- \\
-- \\
--\end{array}$ & $\begin{array}{l}-- \\
-- \\
-- \\
-- \\
--\end{array}$ & $\begin{array}{l}-- \\
-- \\
-- \\
-- \\
--\end{array}$ & $\begin{array}{l}5.2 \\
5.3 \\
5.7 \\
5.9 \\
6.3\end{array}$ \\
\hline $\begin{array}{l}05-14-85 \\
05-21-85 \\
05-28-85 \\
06-04-85 \\
06-11-85\end{array}$ & $\begin{array}{l}1.0 \\
<.1 \\
-- \\
2.0 \\
--\end{array}$ & $\begin{array}{l}-- \\
-- \\
-- \\
-- \\
--\end{array}$ & $\begin{array}{l}40 \\
35 \\
-- \\
36 \\
--\end{array}$ & $\begin{array}{l}-- \\
-- \\
-- \\
-- \\
--\end{array}$ & $\begin{array}{l}-- \\
-- \\
-- \\
-- \\
--\end{array}$ & $\begin{array}{l}-- \\
-- \\
-- \\
-- \\
--\end{array}$ & $\begin{array}{l}-- \\
-- \\
-- \\
-- \\
--\end{array}$ & $\begin{array}{l}-- \\
-- \\
-- \\
-- \\
--\end{array}$ & $\begin{array}{l}-- \\
-- \\
-- \\
-- \\
--\end{array}$ & $\begin{array}{c}6.3 \\
6.3 \\
-- \\
7.1 \\
--\end{array}$ \\
\hline $\begin{array}{l}06-18-85 \\
06-25-85 \\
07-02-85 \\
07-10-85 \\
07-16-85\end{array}$ & $\begin{array}{c}1.0 \\
-- \\
<.1 \\
-- \\
<.1\end{array}$ & $\begin{array}{l}-- \\
-- \\
-- \\
-- \\
--\end{array}$ & $\begin{array}{r}41 \\
-- \\
52 \\
-- \\
170\end{array}$ & $\begin{array}{l}-- \\
-- \\
-- \\
--\end{array}$ & $\begin{array}{l}-- \\
-- \\
-- \\
-- \\
--\end{array}$ & $\begin{array}{l}-- \\
-- \\
-- \\
-- \\
--\end{array}$ & $\begin{array}{l}-- \\
-- \\
-- \\
-- \\
--\end{array}$ & $\begin{array}{l}-- \\
-- \\
-- \\
-- \\
--\end{array}$ & $\begin{array}{l}-- \\
-- \\
-- \\
-- \\
--\end{array}$ & $\begin{array}{l}7.1 \\
-- \\
-- \\
-- \\
7.5\end{array}$ \\
\hline $\begin{array}{l}07-23-85 \\
07-30-85 \\
08-08-85 \\
08-13-85 \\
08-20-85\end{array}$ & $\begin{array}{l}-\overline{1} \\
-\overline{1.0} \\
--\end{array}$ & $\begin{array}{l}-- \\
-- \\
-- \\
--\end{array}$ & $\begin{array}{r}-- \\
170 \\
-- \\
170 \\
--\end{array}$ & $\begin{array}{l}-- \\
-- \\
-- \\
--\end{array}$ & $\begin{array}{l}-- \\
-- \\
-- \\
--\end{array}$ & $\begin{array}{l}-- \\
-- \\
-- \\
--\end{array}$ & $\begin{array}{l}-- \\
-- \\
-- \\
--\end{array}$ & $\begin{array}{l}-- \\
-- \\
-- \\
--\end{array}$ & $\begin{array}{l}-- \\
-- \\
-- \\
--\end{array}$ & $\begin{array}{l}-- \\
-- \\
-- \\
7.8\end{array}$ \\
\hline $\begin{array}{l}08-27-85 \\
09-04-85 \\
09-10-85\end{array}$ & $\begin{array}{l}2.0 \\
-- \\
--\end{array}$ & $\begin{array}{l}-- \\
-- \\
--\end{array}$ & $\begin{array}{l}52 \\
-- \\
--\end{array}$ & -- & $\begin{array}{l}-- \\
-- \\
--\end{array}$ & $\begin{array}{l}-- \\
-- \\
--\end{array}$ & $\begin{array}{l}-- \\
-- \\
--\end{array}$ & $\begin{array}{l}-- \\
-- \\
--\end{array}$ & $\begin{array}{l}-- \\
-- \\
--\end{array}$ & $\begin{array}{l}9.3 \\
-- \\
--\end{array}$ \\
\hline
\end{tabular}

Analysis was used in calculation of volume-weighted base flow stream chemistry.

2 Sample filtered through 0.1 micrometer pore filter.

3 Anomalous value that contributed to cation-anion balance difference of greater than 10 percent; value discarded in data analysis. 
Table 12.--Physical characteristics and concentrations of chemical constituents of streamflow at station number 01174035, Brooks Pond tributary near Petersham, Massachusetts

$\left[\mathrm{ft}^{3} / \mathrm{s}\right.$, cubic feet per second; $\mu \mathrm{S} / \mathrm{cm}$, microsiemens per centimeter at 25 degrees Celsius; ${ }^{\circ} \mathrm{C}$, degrees Celsius; $\mathrm{mg} / \mathrm{L}$, milligrams per liter, $\mathrm{mg} / \mathrm{L}$, micrograms per liter, <, less than; dashes indicate not analyzed]

\begin{tabular}{|c|c|c|c|c|c|c|c|c|c|}
\hline Date & Time & $\begin{array}{l}\text { Stream- } \\
\text { flow, } \\
\text { instan- } \\
\text { taneous } \\
\left(\mathrm{ft}^{3} / \mathrm{s}\right)\end{array}$ & $\begin{array}{l}\text { Spe- } \\
\text { cific } \\
\text { con- } \\
\text { duct- } \\
\text { ance } \\
(\mu \mathrm{s} / \mathrm{cm})\end{array}$ & $\begin{array}{c}\mathrm{pH} \\
\text { (stand- } \\
\text { ard } \\
\text { units) }\end{array}$ & $\begin{array}{l}\text { Temper- } \\
\text { ature } \\
\left({ }^{\circ} \mathrm{C}\right)\end{array}$ & $\begin{array}{c}\text { Hard- } \\
\text { ness } \\
\text { (mg/L } \\
\text { as } \\
\mathrm{CaCO}_{3} \text { ) }\end{array}$ & $\begin{array}{c}\text { Calcium, } \\
\text { dis- } \\
\text { solved } \\
\text { (mg/L } \\
\text { as Ca) }\end{array}$ & $\begin{array}{c}\text { Magne- } \\
\text { sium, } \\
\text { dis- } \\
\text { solved } \\
\text { (mg/L } \\
\text { as Mg) }\end{array}$ & $\begin{array}{l}\text { Sodium, } \\
\text { dis- } \\
\text { solved } \\
\text { (mg/L } \\
\text { as } \mathrm{Na} \text { ) }\end{array}$ \\
\hline $02-16-84$ & $13: 00$ & 33 & -- & 5.6 & -- & 7 & 1.8 & 0.63 & 2.7 \\
\hline $04-06-84$ & $12: 00$ & -- & 30 & 5.6 & -- & 6 & 1.6 & .48 & 2.6 \\
\hline $05-30-84$ & $15: 15$ & -- & 28 & 5.4 & 12.0 & 5 & 1.5 & .42 & 2.0 \\
\hline $05-30-84^{1}$ & $15: 20$ & -- & -- & -- & -- & -- & -- & -- & -- \\
\hline $08-28-84$ & $11: 40$ & -- & 56 & 6.2 & 19.0 & -- & -- & -- & -- \\
\hline $09-11-84$ & $10: 45$ & .02 & 61 & 6.1 & 16.5 & 9 & 2.5 & .79 & 6.2 \\
\hline $10-02-84$ & - & -- & 63 & 6.2 & 9.0 & - & - & -- & -- \\
\hline $10-17-84$ & $07: 20$ & -- & 60 & 6.0 & 10.0 & -- & -- & -- & -- \\
\hline $12-19-84$ & $13: 45$ & 1.7 & 52 & 5.9 & 3.0 & 10 & 2.6 & .89 & 3.9 \\
\hline $04-30-85$ & $10: 15$ & -- & 46 & 6.0 & 14.0 & - & -- & -- & -- \\
\hline $05-28-85$ & $10: 00$ & -- & 50 & 6.0 & 18.0 & -- & -- & -- & -- \\
\hline $06-25-85$ & $15: 45$ & -- & 50 & 6.1 & 18.0 & -- & -- & -- & -- \\
\hline $07-23-85$ & $11: 00$ & -- & 58 & 6.2 & 18.0 & -- & -- & -- & -- \\
\hline $08-08-85$ & $11: 15$ & -- & 59 & 6.3 & 19.5 & -- & -- & -- & -- \\
\hline $08-27-85$ & $12: 30$ & 1.3 & 40 & 6.1 & 20.5 & 8 & 2.1 & .61 & 3.0 \\
\hline
\end{tabular}

See footnote at end of table.

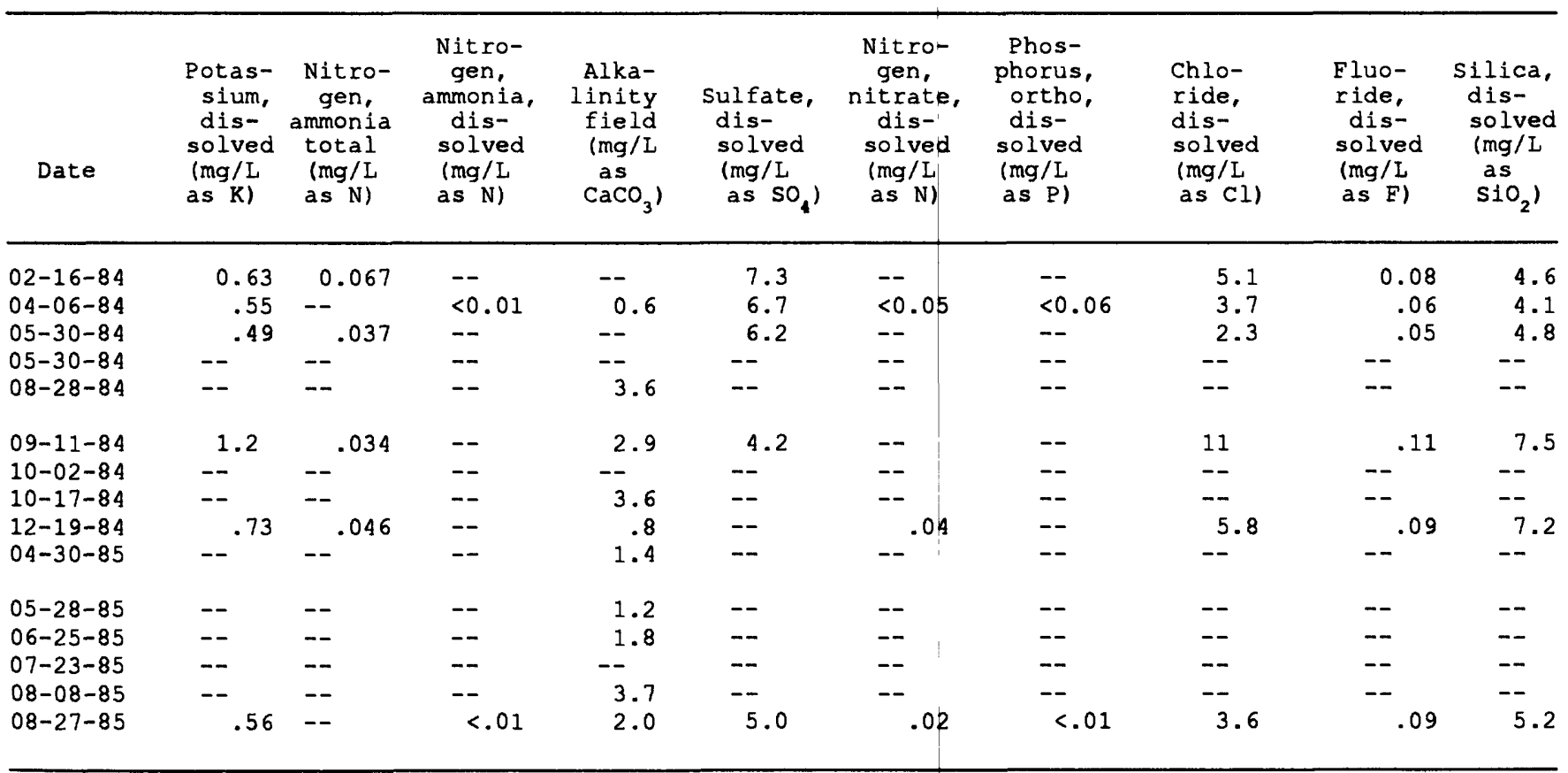


Table 12.--Physical characteristics and concentrations of chemical constituents of streamflow at station number 01174035, Brooks Pond tributary near Petersham, Massachusetts--Continued

\begin{tabular}{|c|c|c|c|c|c|c|c|c|c|c|}
\hline Date & $\begin{array}{l}\text { Solids, } \\
\text { sum of } \\
\text { consti- } \\
\text { tuents, } \\
\text { dis- } \\
\text { solved } \\
\text { (mg/I) }\end{array}$ & $\begin{array}{l}\text { Alum- } \\
\text { inum, } \\
\text { total } \\
\text { recov- } \\
\text { erable } \\
(\mu \mathrm{g} / \mathrm{L} \\
\text { as Al) }\end{array}$ & $\begin{array}{l}\text { Alum- } \\
\text { inum, } \\
\text { dis- } \\
\text { solved } \\
(\mu \mathrm{g} / \mathrm{L} \\
\text { as AI) }\end{array}$ & $\begin{array}{c}\text { Arsenic, } \\
\text { dis- } \\
\text { solved } \\
\text { ( } \mu \text { g/L } \\
\text { as As) }\end{array}$ & $\begin{array}{c}\text { Arsenic, } \\
\text { total } \\
(\mu g / L \\
\text { as As) }\end{array}$ & $\begin{array}{l}\text { Copper, } \\
\text { total } \\
\text { recov- } \\
\text { erable } \\
(\mu g / L \\
\text { as } C u)\end{array}$ & $\begin{array}{l}\text { Copper, } \\
\text { dis- } \\
\text { solved } \\
\text { ( } \mu g / L \\
\text { as } \mathrm{Cu} \text { ) }\end{array}$ & $\begin{array}{l}\text { Iron, } \\
\text { total } \\
\text { recov- } \\
\text { erable } \\
(\mu \mathrm{g} / \mathrm{L} \\
\text { as } \mathrm{Fe})\end{array}$ & $\begin{array}{l}\text { Iron, } \\
\text { dis- } \\
\text { solved } \\
(\mu \mathrm{g} / \mathrm{L} \\
\text { as } \mathrm{Fe})\end{array}$ & $\begin{array}{l}\text { Lead, } \\
\text { total } \\
\text { recov- } \\
\text { erable } \\
(\mu \mathrm{g} / \mathrm{L} \\
\text { as } \mathrm{Pb})\end{array}$ \\
\hline $02-16-84$ & -- & 220 & 220 & $<1$ & 1 & 1 & 0.8 & 290 & 73 & $<1$ \\
\hline $04-06-84$ & 20 & $\ldots$ & - & - & - & - & - & - & 24 & - \\
\hline $05-30-84$ & - & -- & -- & -- & -- & -- & -- & - & 99 & - \\
\hline $05-30-84$ & - & - & ${ }^{1} 230$ & -- & -- & -- & - & - & ${ }^{1} 86$ & -- \\
\hline $08-28-84$ & -- & -- & -- & -- & -- & - & -- & - & - & -- \\
\hline $09-11-84$ & -- & 90 & 50 & $<1$ & $<1$ & 2 & $<1$ & 810 & 350 & 1 \\
\hline $10-02-84$ & -- & -- & - & - & -- & - & - & $\ldots$ & $\ldots$ & - \\
\hline $10-17-84$ & -- & -- & - & -- & -- & -- & -- & $\ldots$ & -- & -- \\
\hline $12-19-84$ & -- & $<10$ & $<10$ & -- & - & 1 & .2 & 320 & 160 & 2 \\
\hline $04-30-85$ & -- & -- & - & -- & -- & - & - & - & - & -- \\
\hline $05-28-85$ & - & -- & -- & -- & -- & - & -- & - & - & -- \\
\hline $06-25-85$ & - & - & -- & -- & -- & -- & -- & - & -- & -- \\
\hline $07-23-85$ & -- & -- & -- & -- & -- & - & -- & -- & -- & - \\
\hline $08-08-85$ & -- & -- & -- & -- & -- & -- & -- & -- & -- & -- \\
\hline $08-27-85$ & 22 & -- & 70 & - & - & -- & -- & - & 390 & -- \\
\hline
\end{tabular}

See footnote at end of table.

\begin{tabular}{|c|c|c|c|c|c|c|c|c|c|c|}
\hline $05-30-84$ & -- & $-\infty$ & 50 & -- & -- & -- & -- & -- & 5.0 & -- \\
\hline $05-30-84$ & -- & - & ${ }^{1} 51$ & -- & -- & -- & -- & -- & -- & -- \\
\hline $08-28-84$ & -- & -- & -- & -- & -- & -- & -- & -- & -- & -- \\
\hline $09-11-84$ & .4 & 150 & 96 & $<.1$ & $<.1$ & $<1$ & $<1$ & $<1$ & -. & 6.4 \\
\hline $10-02-84$ & -- & - & -- & - & - & - & -- & -- & -- & -- \\
\hline $10-17-84$ & -- & - & -- & -- & -- & - & -- & -- & $\ldots$ & -- \\
\hline $06-25-85$ & - & - & - & -- & -- & - & -- & - & -- & -- \\
\hline $07-23-85$ & -- & -- & -- & -- & -- & -- & -- & -- & -- & -- \\
\hline $08-08-85$ & - & - & -- & -- & -- & -- & -- & - & -- & -- \\
\hline $08-27-85$ & -- & -- & 33 & -- & -- & -- & -- & -- & -- & 7.2 \\
\hline
\end{tabular}

1 Sample filtered through 0.1 micrometer pore filter. 
Table 13.--Physical characteristics and concentrations of chemical constituents of streamflow at station number 01174040, East Branch Fever Brook below Brooks Pond near Petersham, Massachusetts

$\left[\mathrm{ft}^{3} / \mathrm{s}\right.$, cubic feet per second; $\mu \mathrm{S} / \mathrm{cm}$, microsiemens per centimeter at 25 degrees Celsius; ${ }^{\circ} \mathrm{C}$, degrees Celsius; $\mathrm{mg} / \mathrm{L}$. milligrams per liter; $\mu \mathrm{g} / \mathrm{L}$, micrograms per liter, $<$, less than; dashes indicate not analyzed]

\begin{tabular}{|c|c|c|c|c|c|c|c|c|c|}
\hline Date & Time & $\begin{array}{l}\text { Stream- } \\
\text { flow, } \\
\text { instan- } \\
\text { taneous } \\
\left(\mathrm{ft}^{3} / \mathrm{s}\right)\end{array}$ & $\begin{array}{l}\text { Spe- } \\
\text { cific } \\
\text { con- } \\
\text { duct- } \\
\text { ance } \\
(\mu s / \mathrm{cm})\end{array}$ & $\begin{array}{c}\mathrm{pH} \\
\text { (stand- } \\
\text { ard } \\
\text { units) }\end{array}$ & $\begin{array}{l}\text { Temper- } \\
\text { ature } \\
\left({ }^{\circ} \mathrm{C}\right)\end{array}$ & $\begin{array}{l}\text { Hard- } \\
\text { ness } \\
\text { (mg/L } \\
\text { as } \\
\mathrm{CaCO}_{3} \text { ) } \\
\end{array}$ & $\begin{array}{l}\text { Calcium, } \\
\text { dis- } \\
\text { solved } \\
\text { (mg/I } \\
\text { as Ca) }\end{array}$ & $\begin{array}{c}\text { Magne- } \\
\text { sium, } \\
\text { dis- } \\
\text { solved } \\
\text { (mg/L } \\
\text { as Mg) }\end{array}$ & $\begin{array}{l}\text { Sodium, } \\
\text { dis- } \\
\text { solved } \\
\text { (mg/L } \\
\text { as } \mathrm{Na} \text { ) }\end{array}$ \\
\hline $02-16-84$ & $14: 10$ & 65 & -- & 5.6 & 0.5 & 7 & 1.9 & 0.66 & 4.5 \\
\hline $03-27-84$ & $14: 20$ & -- & -- & -- & -- & -- & -- & -- & -- \\
\hline $04-06-84$ & $12: 00$ & -- & 48 & 5.6 & -- & 7 & 1.7 & .56 & 5.0 \\
\hline $04-06-84$ & $14: 10$ & -- & 48 & 5.6 & -- & -- & -- & -- & -- \\
\hline $04-10-84$ & $09: 20$ & -- & -- & -- & -- & -- & -- & -- & -- \\
\hline $04-17-84$ & $13: 40$ & -- & -- & -- & -- & -- & -- & -- & -- \\
\hline $05-30-84$ & $15: 00$ & -- & 35 & 5.7 & 14.5 & 6 & 1.5 & .43 & 3.8 \\
\hline $05-30-84^{1}$ & $15: 10$ & -- & -- & -- & -- & -- & -- & -- & -- \\
\hline $08-28-84$ & $11: 50$ & -- & 42 & 5.6 & 23.0 & -- & -- & -- & -- \\
\hline $09-11-84$ & $11: 45$ & -- & 44 & 5.7 & 20.0 & 7 & 1.7 & .55 & 5.1 \\
\hline $10-02-84$ & -- & -- & 49 & 5.7 & 9.0 & -- & -- & -- & -- \\
\hline $12-19-84$ & $12: 15$ & 2.2 & 63 & 5.8 & 4.0 & 9 & 2.2 & .83 & 6.7 \\
\hline $04-30-85$ & $10: 30$ & -- & 66 & 5.6 & 16.5 & -- & -- & -- & -- \\
\hline $05-28-85$ & $10: 30$ & -- & 70 & 5.6 & 20.5 & -- & -- & -- & -- \\
\hline $06-25-85$ & $15: 30$ & -- & 76 & 5.5 & 21.5 & -- & -- & -- & -- \\
\hline $07-23-85$ & $11: 20$ & -- & 72 & 5.2 & 24.5 & -- & -- & -- & -- \\
\hline $08-08-85$ & $11: 00$ & -- & 78 & 5.3 & 22.0 & -- & -- & -- & -- \\
\hline $08-27-85$ & $14: 00$ & 4.0 & 75 & 5.3 & 20.5 & 9 & 2.3 & .71 & 8.3 \\
\hline
\end{tabular}

See footnote at end of table.

\begin{tabular}{|c|c|c|c|c|c|c|c|c|c|c|}
\hline Date & $\begin{array}{l}\text { Potas- } \\
\text { sium, } \\
\text { dis- } \\
\text { solved } \\
\text { (mg/L } \\
\text { as K) }\end{array}$ & $\begin{array}{l}\text { Nitro- } \\
\text { gen, } \\
\text { ammonia } \\
\text { total } \\
\text { (mg/L } \\
\text { as N) }\end{array}$ & $\begin{array}{l}\text { Nitro- } \\
\text { gen, } \\
\text { ammonia, } \\
\text { dis- } \\
\text { solved } \\
\text { (mg/L } \\
\text { as N) }\end{array}$ & $\begin{array}{l}\text { Alka- } \\
\text { linity } \\
\text { field } \\
\text { (mg/L } \\
\text { as } \\
\mathrm{CaCO}_{3} \text { ) }\end{array}$ & $\begin{array}{l}\text { Sulfate, } \\
\text { dis- } \\
\text { solved } \\
\text { (mg/L } \\
\text { as } \mathrm{SO}_{4} \text { ) }\end{array}$ & $\begin{array}{l}\text { Nitro- } \\
\text { gen, } \\
\text { nitrate, } \\
\text { dis- } \\
\text { solved } \\
\text { (mg/L } \\
\text { as } \mathrm{N} \text { ) }\end{array}$ & $\begin{array}{l}\text { Phos- } \\
\text { phorus, } \\
\text { ortho, } \\
\text { dis- } \\
\text { solved } \\
\text { (mg/L } \\
\text { as P) }\end{array}$ & $\begin{array}{l}\text { Chlo- } \\
\text { ride, } \\
\text { dis- } \\
\text { solved } \\
\text { (mg/L } \\
\text { as Cl) }\end{array}$ & $\begin{array}{l}\text { Fluo- } \\
\text { ride, } \\
\text { dis- } \\
\text { solved } \\
\text { (mg/I } \\
\text { as F) }\end{array}$ & $\begin{array}{l}\text { Silica, } \\
\text { dis- } \\
\text { solved } \\
\text { (mg/L } \\
\text { as } \\
\mathrm{SiO}_{2} \text { ) }\end{array}$ \\
\hline $02-16-84$ & 0.61 & $<0.001$ & -- & 1 & 7.1 & -- & -- & 7.1 & 0.09 & 4.8 \\
\hline $03-27-84$ & -- & -- & -- & -- & -- & -- & -- & -- & -- & -- \\
\hline $04-06-84$ & .55 & -- & 1.10 & -- & 6.4 & $<0.05$ & $<0.06$ & 8.2 & .06 & 3.9 \\
\hline $04-06-84$ & -- & -- & -- & .5 & -- & -- & -- & -- & -- & -- \\
\hline $04-10-84$ & -- & -- & -- & -- & -- & -- & -- & -- & -- & -- \\
\hline $04-17-84$ & -- & -- & -- & -- & -- & -- & -- & -- & -- & -- \\
\hline $05-30-84$ & .49 & .034 & -- & -- & 5.4 & -- & -- & 5.9 & .05 & 2.7 \\
\hline $05-30-84$ & -- & -- & -- & -- & -- & -- & -- & -- & -- & -- \\
\hline $08-28-84$ & -- & -- & -- & 2.4 & -- & -- & -- & -- & -- & -- \\
\hline $09-11-84$ & .34 & .061 & -- & .7 & 4.1 & -- & -- & 8.0 & .09 & .8 \\
\hline $10-02-84$ & -- & -- & -- & -- & -- & -- & -- & -- & -- & -- \\
\hline $12-19-84$ & .86 & .065 & -- & 1.8 & 5.2 & -- & $<.01$ & 11 & -- & 3.5 \\
\hline $04-30-85$ & -- & -- & -- & .7 & -- & -- & -- & -- & -- & -- \\
\hline $05-28-85$ & -- & -- & -- & .8 & -- & -- & -- & -- & -- & -- \\
\hline $06-25-85$ & -- & -- & -- & .4 & -- & -- & -- & -- & -- & -- \\
\hline $07-23-85$ & -- & -- & -- & -- & -- & -- & -- & -- & -- & -- \\
\hline $08-08-85$ & -- & -- & -- & .7 & -- & -- & -- & -- & -- & -- \\
\hline $08-27-85$ & .37 & -- & $<.01$ & 1.1 & 5.3 & .02 & $<.01$ & 14 & .14 & 1.6 \\
\hline
\end{tabular}


Table 13.--Physical characteristics and concentrations of chemical constituents of streamflow at station number 01174040, East Branch Fever Brook below Brooks Pond near Petersham, Massachusetts--Continued

\begin{tabular}{|c|c|c|c|c|c|c|c|c|c|c|}
\hline Date & $\begin{array}{l}\text { Solids, } \\
\text { sum of } \\
\text { consti- } \\
\text { tuents, } \\
\text { dis- } \\
\text { solved } \\
\text { (mg/L) }\end{array}$ & $\begin{array}{l}\text { Alum- } \\
\text { inum, } \\
\text { total } \\
\text { recov- } \\
\text { erable } \\
(\mu \mathrm{g} / \mathrm{L} \\
\text { as Al) }\end{array}$ & $\begin{array}{l}\text { Alum- } \\
\text { inum, } \\
\text { dis- } \\
\text { solved } \\
\text { ( } \mu \text { g/L } \\
\text { as Al) }\end{array}$ & $\begin{array}{c}\text { Arsenic, } \\
\text { dis- } \\
\text { solved } \\
\text { ( } \mu \mathrm{g} / \mathrm{L} \\
\text { as As) }\end{array}$ & $\begin{array}{l}\text { Arsenic, } \\
\text { total } \\
\text { ( } \mu g / L \\
\text { as As) }\end{array}$ & $\begin{array}{c}\text { Copper, } \\
\text { total } \\
\text { recov- } \\
\text { erable } \\
(\mu g / L \\
\text { as } \mathrm{Cu})\end{array}$ & $\begin{array}{l}\text { Copper, } \\
\text { dis- } \\
\text { solved } \\
\text { ( } \mu g / L \\
\text { as } \mathrm{Cu} \text { ) }\end{array}$ & $\begin{array}{l}\text { Iron, } \\
\text { total } \\
\text { recov- } \\
\text { erable } \\
\text { ( } \mu \mathrm{g} / \mathrm{L} \\
\text { as } \mathrm{Fe})\end{array}$ & $\begin{array}{l}\text { Iron, } \\
\text { dis- } \\
\text { solved } \\
(\mu \mathrm{g} / \mathrm{L} \\
\text { as } \mathrm{Fe})\end{array}$ & $\begin{array}{l}\text { Lead, } \\
\text { total } \\
\text { recov- } \\
\text { erable } \\
(\mu g / L \\
\text { as } \mathrm{Pb})\end{array}$ \\
\hline $\begin{array}{l}02-16-84 \\
03-27-84 \\
04-06-84 \\
04-06-84 \\
04-10-84\end{array}$ & $\begin{array}{l}28 \\
-- \\
-- \\
-- \\
--\end{array}$ & $\begin{array}{r}170 \\
-- \\
-- \\
-- \\
--\end{array}$ & $\begin{array}{r}100 \\
20 \\
-- \\
80 \\
<10\end{array}$ & $\begin{array}{l}<1 \\
-- \\
-- \\
-- \\
--\end{array}$ & $\begin{array}{l}<1 \\
-- \\
-- \\
-- \\
--\end{array}$ & $\begin{array}{l}2 \\
-- \\
-- \\
-- \\
--\end{array}$ & $\begin{array}{l}1.3 \\
-- \\
-- \\
-- \\
--\end{array}$ & $\begin{array}{c}330 \\
-- \\
-- \\
-- \\
--\end{array}$ & $\begin{array}{r}110 \\
38 \\
36 \\
40 \\
35\end{array}$ & $\begin{array}{l}2 \\
-- \\
-- \\
-- \\
--\end{array}$ \\
\hline $\begin{array}{l}04-17-84 \\
05-30-84 \\
05-30-84 \\
08-28-84 \\
09-11-84\end{array}$ & $\begin{array}{l}-- \\
-- \\
-- \\
-- \\
--\end{array}$ & $\begin{array}{l}-- \\
-- \\
-- \\
--\end{array}$ & $\begin{array}{r}20 \\
\mathrm{I}_{20} \\
\overline{50}\end{array}$ & $\begin{array}{l}-- \\
-- \\
-- \\
-- \\
<1\end{array}$ & $\begin{array}{c}-- \\
-- \\
-- \\
-- \\
1\end{array}$ & $\begin{array}{l}-- \\
-- \\
-- \\
-- \\
2\end{array}$ & $\begin{array}{l}-- \\
-- \\
-- \\
-- \\
2.6\end{array}$ & $\begin{array}{l}-- \\
-- \\
-- \\
-- \\
350\end{array}$ & $\begin{array}{r}63 \\
130 \\
45 \\
-- \\
180\end{array}$ & $\begin{array}{l}-- \\
-- \\
-- \\
-- \\
<1\end{array}$ \\
\hline $\begin{array}{l}10-02-84 \\
12-19-84 \\
04-30-85 \\
05-28-85 \\
06-25-85\end{array}$ & $\begin{array}{l}-- \\
32 \\
-- \\
-- \\
--\end{array}$ & $\begin{array}{r}-- \\
130 \\
-- \\
-- \\
--\end{array}$ & $\begin{array}{c}-- \\
<10 \\
-- \\
-- \\
--\end{array}$ & $\begin{array}{l}-- \\
-- \\
-- \\
-- \\
--\end{array}$ & $\begin{array}{l}-- \\
-- \\
-- \\
-- \\
--\end{array}$ & $\begin{array}{c}-- \\
1 \\
-- \\
-- \\
--\end{array}$ & $\begin{array}{l}-- \\
-- \\
-- \\
--\end{array}$ & $\begin{array}{r}-- \\
350 \\
-- \\
-- \\
--\end{array}$ & $\begin{array}{r}160 \\
-- \\
-- \\
--\end{array}$ & $\begin{array}{r}-- \\
2 \\
-- \\
-- \\
--\end{array}$ \\
\hline $\begin{array}{l}07-23-85 \\
08-08-85 \\
08-27-85\end{array}$ & $-\overline{34}$ & $\begin{array}{l}-- \\
--\end{array}$ & $\begin{array}{l}-- \\
--\end{array}$ & -- & $\begin{array}{l}-- \\
-- \\
--\end{array}$ & $\begin{array}{l}-- \\
--\end{array}$ & $\begin{array}{l}-- \\
--\end{array}$ & $\begin{array}{l}-- \\
-- \\
--\end{array}$ & $\frac{--}{160}$ & $\begin{array}{l}-- \\
-- \\
--\end{array}$ \\
\hline
\end{tabular}

See footnote at end of table.

\begin{tabular}{|c|c|c|c|c|c|c|c|c|c|c|}
\hline Date & $\begin{array}{c}\text { Lead, } \\
\text { dis- } \\
\text { solved } \\
(\mu \mathrm{g} / \mathrm{L} \\
\text { as } \mathrm{Pb})\end{array}$ & $\begin{array}{l}\text { Manga- } \\
\text { nese, } \\
\text { total } \\
\text { recov- } \\
\text { erable } \\
\text { ( } \mu g / I \\
\text { as } \mathrm{Mn} \text { ) }\end{array}$ & $\begin{array}{l}\text { Manga- } \\
\text { nese, } \\
\text { dis- } \\
\text { solved } \\
(\mu g / L \\
\text { as } M n)\end{array}$ & $\begin{array}{c}\text { Mercury, } \\
\text { total } \\
\text { recov- } \\
\text { erable } \\
\text { ( } \mu g / \mathrm{L} \\
\text { as } \mathrm{Hg})\end{array}$ & $\begin{array}{c}\text { Mercury, } \\
\text { dis- } \\
\text { solved } \\
(\mu g / L \\
\text { as } \mathrm{Hg})\end{array}$ & $\begin{array}{l}\text { Sele- } \\
\text { nium, } \\
\text { total } \\
\text { ( } \mu \mathrm{g} / \mathrm{L} \\
\text { as } \mathrm{Se})\end{array}$ & $\begin{array}{l}\text { Sele- } \\
\text { nium, } \\
\text { dis- } \\
\text { solved } \\
\text { ( } \mu \text { g/L } \\
\text { as } S e)\end{array}$ & $\begin{array}{l}\text { Vana- } \\
\text { dium, } \\
\text { dis- } \\
\text { solved } \\
(\mu g / L \\
\text { as V) }\end{array}$ & $\begin{array}{c}\text { Carbon, } \\
\text { organic } \\
\text { total } \\
\text { (mg/L } \\
\text { as C) }\end{array}$ & $\begin{array}{c}\text { Carbon, } \\
\text { organic, } \\
\text { dis- } \\
\text { solved } \\
\text { (mg/L } \\
\text { as C) }\end{array}$ \\
\hline $02-16-84$ & 1.0 & 110 & 100 & $<0.1$ & $<0.1$ & $<1$ & $<1$ & 1 & - & -- \\
\hline $03-27-84$ & - & -- & 25 & -- & -- & $\ldots$ & -- & -- & -- & - \\
\hline $04-06-84$ & -- & -- & 53 & -- & -- & - & -- & -- & -- & -- \\
\hline $04-06-84$ & -- & -- & 34 & -- & -- & -- & -- & -- & -- & -- \\
\hline $04-10-84$ & -- & -- & 26 & -- & -- & -- & -- & -- & $-\infty$ & -- \\
\hline $04-17-84$ & -- & -- & 26 & -- & -- & -- & -- & -- & -- & -- \\
\hline $05-30-84$ & -- & -- & 41 & -- & -- & -- & -- & -- & 4.8 & -- \\
\hline $05-30-84$ & -- & -- & ${ }^{1} 12$ & -- & -- & - & -- & -- & -- & -- \\
\hline $08-28-84$ & -- & -- & -- & -- & -- & -- & -- & -- & -- & -- \\
\hline $09-11-84$ & 1.0 & 30 & 31 & $<.1$ & $<.1$ & $<1$ & $<1$ & $<1$ & 8.2 & 8.2 \\
\hline $10-02-84$ & -- & -- & -- & -- & -- & -- & -- & -- & -- & -- \\
\hline $12-19-84$ & .4 & 20 & 28 & -- & -- & - & -- & -- & -- & 7.6 \\
\hline $04-30-85$ & -- & -- & -- & - & -- & - & -- & -- & -- & -- \\
\hline $05-28-85$ & -- & -- & -- & -- & -- & - & -- & -- & -- & -- \\
\hline $06-25-85$ & -- & -- & -- & -- & -- & -- & -- & -- & -- & -- \\
\hline $07-23-85$ & -- & -- & -- & -- & - & -- & -- & -- & -- & -- \\
\hline $08-08-85$ & -- & -- & -- & -- & -- & -- & -- & -- & -- & -- \\
\hline $08-27-85$ & -- & -- & 32 & -- & -- & -- & -- & -- & -- & 7.2 \\
\hline
\end{tabular}

1 Sample filtered through 0.1 micrometer pore filter. 
Table 14.--Physical characteristics and concentrations of chemical constituents of streamflow at station number 01174045, East Branch Fever Brook at West Road near Petersham, Massachusetts

$\left[\mathrm{ft}^{3} / \mathrm{s}\right.$, cubic feet per second; $\mu \mathrm{S} / \mathrm{cm}$, microsiemens per centimeter at 25 degrees Celsius; ${ }^{\circ} \mathrm{C}$, degrees Celsius; mg $/$, milligrams per liter; $\mu \mathrm{g} /$, micrograms per liter; <, less than; dashes indicate not analyzed]

\begin{tabular}{|c|c|c|c|c|c|c|c|c|c|}
\hline Date & Time & $\begin{array}{l}\text { Stream- } \\
\text { flow, } \\
\text { instan- } \\
\text { taneous } \\
\left(\mathrm{ft}^{3} / \mathrm{s}\right)\end{array}$ & $\begin{array}{l}\text { Spe- } \\
\text { cific } \\
\text { con- } \\
\text { duct- } \\
\text { ance } \\
(\mu \mathrm{s} / \mathrm{cm})\end{array}$ & $\begin{array}{l}\mathrm{pH} \\
\text { (stand- } \\
\text { ard } \\
\text { units) }\end{array}$ & $\begin{array}{l}\text { Temper- } \\
\text { ature } \\
\left({ }^{\circ} \mathrm{C}\right)\end{array}$ & $\begin{array}{l}\text { Hard- } \\
\text { ness } \\
(\mathrm{mg} / \mathrm{L} \\
\mathrm{as} \\
\mathrm{CaCO}_{3} \text { ) }\end{array}$ & $\begin{array}{l}\text { Calcium, } \\
\text { dis- } \\
\text { solved } \\
\text { (mg/L } \\
\text { as Ca) }\end{array}$ & $\begin{array}{l}\text { Magne- } \\
\text { sium, } \\
\text { dis- } \\
\text { solved } \\
\text { (mg/L } \\
\text { as Mg) }\end{array}$ & $\begin{array}{l}\text { Sodium, } \\
\text { dis- } \\
\text { solved } \\
\text { (mg/L } \\
\text { as } \mathrm{Na} \text { ) }\end{array}$ \\
\hline $\begin{array}{l}02-16-84 \\
04-06-84 \\
05-30-84 \\
05-30-84 \\
08-28-84\end{array}$ & $\begin{array}{l}11: 45 \\
12: 00 \\
15: 30 \\
15: 40 \\
11: 30\end{array}$ & $\begin{array}{l}90 \\
-- \\
-- \\
-- \\
--\end{array}$ & $\begin{array}{l}-- \\
44 \\
39 \\
-- \\
53\end{array}$ & $\begin{array}{r}5.6 \\
5.5 \\
5.3 \\
- \\
6.0\end{array}$ & $\begin{array}{l}\overline{--} \\
\overline{13} .0 \\
\overline{20} .0\end{array}$ & $\begin{array}{r}8 \\
6 \\
6 \\
-- \\
--\end{array}$ & $\begin{array}{l}2.1 \\
1.6 \\
1.5 \\
-- \\
--\end{array}$ & $\begin{array}{l}0.7 \\
.54 \\
. .48 \\
--\end{array}$ & $\begin{array}{l}6.7 \\
5.1 \\
4.3 \\
-- \\
--\end{array}$ \\
\hline $\begin{array}{l}09-11-84 \\
10-02-84 \\
10-17-84 \\
12-19-84 \\
04-30-85\end{array}$ & $\begin{array}{c}10: 00 \\
-- \\
07: 00 \\
15: 00 \\
09: 45\end{array}$ & ${ }_{--}^{.06}$ & $\begin{array}{l}55 \\
72 \\
56 \\
70 \\
72\end{array}$ & $\begin{array}{l}6.1 \\
5.7 \\
6.0 \\
5.8 \\
5.9\end{array}$ & $\begin{array}{r}18.0 \\
9.0 \\
9.0 \\
3.0 \\
14.5\end{array}$ & $\begin{array}{c}8 \\
-- \\
--\end{array}$ & $\begin{array}{l}2.1 \\
-- \\
-- \\
2.4\end{array}$ & $\begin{array}{l}-.72 \\
-- \\
--^{-87}\end{array}$ & $\begin{array}{l}6.3 \\
-- \\
-- \\
7.7 \\
--\end{array}$ \\
\hline $\begin{array}{l}05-28-85 \\
06-25-85 \\
07-23-85 \\
08-08-85 \\
08-27-85\end{array}$ & $\begin{array}{l}08: 45 \\
16: 00 \\
10: 45 \\
10: 45 \\
11: 00\end{array}$ & $\begin{array}{l}-- \\
-- \\
-- \\
-- \\
6.2\end{array}$ & $\begin{array}{l}69 \\
78 \\
79 \\
81 \\
80\end{array}$ & $\begin{array}{l}5.6 \\
5.7 \\
5.9 \\
5.8 \\
5.6\end{array}$ & $\begin{array}{l}18.5 \\
19.0 \\
19.0 \\
20.0 \\
19.5\end{array}$ & $\begin{array}{l}-- \\
-- \\
-- \\
-- \\
10\end{array}$ & $\begin{array}{l}-- \\
-- \\
-- \\
-- \\
2.5\end{array}$ & $\begin{array}{l}-- \\
-- \\
-- \\
-- \\
\quad .82\end{array}$ & $\begin{array}{l}-- \\
-- \\
-- \\
-- \\
8.9\end{array}$ \\
\hline
\end{tabular}

See footnote at end of table.

\begin{tabular}{|c|c|c|c|c|c|c|c|c|c|c|}
\hline Date & $\begin{array}{l}\text { Potas- } \\
\text { sium, } \\
\text { dis- } \\
\text { solved } \\
\text { (mg/L } \\
\text { as K) }\end{array}$ & $\begin{array}{c}\text { Nitro- } \\
\text { gen, } \\
\text { ammonia } \\
\text { total } \\
\text { (mg/L } \\
\text { as } N \text { ) }\end{array}$ & $\begin{array}{l}\text { Nitro- } \\
\text { gen, } \\
\text { ammonia, } \\
\text { dis- } \\
\text { solved } \\
\text { (mg/L } \\
\text { as N) }\end{array}$ & $\begin{array}{c}\text { Alka- } \\
\text { linity } \\
\text { field } \\
\text { (mg/L } \\
\text { as } \\
\left.\mathrm{CaCO}_{3}\right)\end{array}$ & $\begin{array}{l}\text { Sulfate, } \\
\text { dis- } \\
\text { solved } \\
\text { (mg/L } \\
\text { as } \mathrm{SO}_{4} \text { ) }\end{array}$ & $\begin{array}{c}\text { Nitro- } \\
\text { gen, } \\
\text { nitrate, } \\
\text { dis- } \\
\text { solved } \\
\text { (mg/L } \\
\text { as N) }\end{array}$ & $\begin{array}{l}\text { Phos- } \\
\text { phorus, } \\
\text { ortho, } \\
\text { dis- } \\
\text { solved } \\
\text { (mg/L } \\
\text { as P) }\end{array}$ & $\begin{array}{l}\text { Chlo- } \\
\text { ride, } \\
\text { dis- } \\
\text { solved } \\
\text { (mg/L } \\
\text { as Cl) }\end{array}$ & $\begin{array}{l}\text { Fluo- } \\
\text { ride, } \\
\text { dis- } \\
\text { solved } \\
\text { (mg/L } \\
\text { as F) }\end{array}$ & $\begin{array}{l}\text { Silica, } \\
\text { dis- } \\
\text { solved } \\
\text { (mg/L } \\
\text { as } \\
\mathrm{SiO}_{2} \text { ) }\end{array}$ \\
\hline $02-16-84$ & 0.62 & 0.306 & -- & 1.0 & 7.0 & -- & -- & 13 & 0.07 & 5.2 \\
\hline $04-06-84$ & .54 & -- & 0.31 & .5 & 6.4 & $<0.05$ & $<0.06$ & 8.1 & .05 & 4.2 \\
\hline $05-30-84$ & .51 & .077 & -- & -- & 5.4 & -- & -- & 6.6 & .07 & 3.7 \\
\hline $05-30-84$ & -- & -- & -- & -- & -- & - & -- & -- & -- & -- \\
\hline $08-28-84$ & -- & -- & -- & 3.7 & -- & -- & -- & -- & -- & -- \\
\hline $09-11-84$ & .99 & .022 & -- & 3.8 & 3.0 & - & -- & 10 & .09 & 5.4 \\
\hline $10-02-84$ & -- & -- & -- & -- & -- & - & -- & -- & -- & -- \\
\hline $10-17-84$ & -- & -- & -- & -- & -- & - & -- & -- & -- & -- \\
\hline $12-19-84$ & .87 & .047 & -- & 2.0 & -- & -+ & -- & 15 & -- & 4.8 \\
\hline $04-30-85$ & -- & -- & -- & 1.9 & -- & - & -- & -- & -- & -- \\
\hline $05-28-85$ & -- & -- & -- & 1.4 & -- & - & -- & -- & -- & -- \\
\hline $06-25-85$ & -- & -- & -- & 1.8 & -- & - & -- & -- & -- & -- \\
\hline $07-23-85$ & -- & -- & -- & -- & -- & -- & -- & -- & -- & -- \\
\hline $08-08-85$ & -- & -- & -- & 2.2 & -- & - & -- & -- & -- & -- \\
\hline $08-27-85$ & .73 & -- & .01 & 2.3 & 3.7 & .02 & $<.01$ & 16 & .14 & 2.9 \\
\hline
\end{tabular}




\begin{tabular}{|c|c|c|c|c|c|c|c|c|c|c|}
\hline Date & $\begin{array}{l}\text { Solids, } \\
\text { sum of } \\
\text { consti- } \\
\text { tuents, } \\
\text { dis- } \\
\text { solved } \\
\text { (mg/L) }\end{array}$ & $\begin{array}{l}\text { Alum- } \\
\text { inum, } \\
\text { total } \\
\text { recov- } \\
\text { erable } \\
(\mu g / L \\
\text { as Al) }\end{array}$ & $\begin{array}{l}\text { Alum- } \\
\text { inum, } \\
\text { dis- } \\
\text { solved } \\
(\mu g / L \\
\text { as Al) }\end{array}$ & $\begin{array}{c}\text { Arsenic, } \\
\text { dis- } \\
\text { solved } \\
\text { ( } \mu g / L \\
\text { as As) }\end{array}$ & $\begin{array}{c}\text { Arsenic, } \\
\text { total } \\
\text { ( } \mu g / L \\
\text { as As) }\end{array}$ & $\begin{array}{c}\text { Copper, } \\
\text { total } \\
\text { recov- } \\
\text { erable } \\
(\mu g / L \\
\text { as Cu) }\end{array}$ & $\begin{array}{l}\text { Copper, } \\
\text { dis- } \\
\text { solved } \\
(\mu g / L \\
\text { as } \mathrm{Cu})\end{array}$ & $\begin{array}{l}\text { Iron, } \\
\text { total } \\
\text { recov- } \\
\text { erable } \\
\text { ( } \mu g / L \\
\text { as Fe) }\end{array}$ & $\begin{array}{l}\text { Iron, } \\
\text { dis- } \\
\text { solved } \\
(\mu g / L \\
\text { as } \mathrm{Fe})\end{array}$ & $\begin{array}{l}\text { Lead, } \\
\text { totaI } \\
\text { recov- } \\
\text { erable } \\
(\mu g / L \\
\text { as } \mathrm{Pb})\end{array}$ \\
\hline $02-16-84$ & 36 & 140 & 120 & $<1$ & 1 & 2 & 1.3 & 330 & 110 & 2 \\
\hline $04-06-84$ & 27 & - & -- & -- & - & -- & -- & -- & 37 & -- \\
\hline $05-30-84$ & -- & -- & -- & -- & -- & $-\ldots$ & -- & -- & 180 & -- \\
\hline $05-30-84$ & -- & -- & ${ }^{1} 100$ & -- & -- & -- & -- & -- & 1130 & -- \\
\hline $08-28-84$ & -- & - & -- & -- & -- & -- & -- & -- & -- & -- \\
\hline $09-11-84$ & -- & 50 & 30 & $<1$ & $<1$ & 1 & .5 & 800 & 510 & 1 \\
\hline $10-02-84$ & -- & -- & -- & -- & -- & -- & -- & -- & -- & -- \\
\hline $10-17-84$ & -- & -- & -- & -- & -- & -- & -- & -- & -- & -- \\
\hline $12-19-84$ & -- & 100 & $<10$ & -- & -- & 1 & .6 & 450 & 290 & 3 \\
\hline $04-30-85$ & -- & - & - & -- & -- & -- & -- & -- & -- & -- \\
\hline $05-28-85$ & -- & -- & -- & -- & -- & -- & -- & -- & -- & -- \\
\hline $06-25-85$ & -- & -- & -- & -- & -- & -- & -- & -- & -- & -- \\
\hline $07-23-85$ & -- & -- & -- & -- & -- & -- & -- & -- & -- & -- \\
\hline $08-08-85$ & -- & -- & -- & -- & -- & -- & -- & -- & -- & -- \\
\hline $08-27-85$ & 38 & -- & 70 & -- & -- & -- & -- & -- & 510 & -- \\
\hline
\end{tabular}

See footnote at end of table.

\begin{tabular}{|c|c|c|c|c|c|c|c|c|c|c|}
\hline $05-30-84$ & -- & -- & 40 & -- & -- & -- & -- & -- & 5.5 & -- \\
\hline $05-30-84$ & -- & -- & ${ }^{1} 37$ & -- & -- & -- & -- & -- & -- & -- \\
\hline $08-28-84$ & -- & -- & -- & -- & -- & -- & -- & -- & -- & -- \\
\hline $09-11-84$ & 1.7 & 20 & 15 & $<.1$ & $<.1$ & $<1$ & $<1$ & $<1$ & -- & 7.1 \\
\hline $10-02-84$ & -- & -- & -- & -- & -- & -- & -- & -- & -- & - \\
\hline $06-25-85$ & -- & -- & -- & -- & -- & -- & -- & -- & -- & -- \\
\hline $07-23-85$ & -- & -- & -- & -- & -- & -- & -- & -- & -- & -- \\
\hline $08-08-85$ & -- & -- & -- & -- & -- & -- & -- & -- & -- & -- \\
\hline $08-27-85$ & -- & -- & 88 & -- & -- & -- & -- & -- & -- & 8.6 \\
\hline
\end{tabular}

1 Sample filtered through 0.1 micrometer pore filter. 
Table 15.--Physical characteristics and concentrations of chemical constituents of ground water at station number 422906072124301, Fever Brook till well near Petersham, Massachusetts

[ $\mu \mathrm{S} / \mathrm{cm}$, microsiemens per centimeter at 25 degrees Celsius; ${ }^{\circ} \mathrm{C}$, degrees Celsius; $\mathrm{mg} / \mathrm{L}$, milligrams per liter; $\mu \mathrm{g} / \mathrm{L}$, micrograms per liter, <, less than; dashes indicate not analyzed]

\begin{tabular}{|c|c|c|c|c|c|c|c|c|c|}
\hline Date & Time & $\begin{array}{l}\text { Spe- } \\
\text { cific } \\
\text { con- } \\
\text { duct- } \\
\text { ance } \\
(\mu \mathrm{s} / \mathrm{cm})\end{array}$ & $\begin{array}{l}\mathrm{pH} \\
\text { (stand- } \\
\text { ard } \\
\text { units) }\end{array}$ & $\begin{array}{l}\text { Temper- } \\
\text { ature } \\
\left({ }^{\circ} \mathrm{C}\right)\end{array}$ & $\begin{array}{l}\text { Hard- } \\
\text { ness } \\
(\mathrm{mg} / \mathrm{L} \\
\mathrm{as} \\
\left.\mathrm{CaCO}_{3}\right)\end{array}$ & $\begin{array}{l}\text { Calcium, } \\
\text { dis- } \\
\text { solved } \\
\text { (mg/L } \\
\text { as Ca) }\end{array}$ & $\begin{array}{l}\text { Magne- } \\
\text { sium, } \\
\text { dis- } \\
\text { solved } \\
\text { (mg/L } \\
\text { as Mg) }\end{array}$ & $\begin{array}{l}\text { Sodium, } \\
\text { dis- } \\
\text { solved } \\
\text { (mg/L } \\
\text { as Na) }\end{array}$ & $\begin{array}{l}\text { Potas- } \\
\text { sium, } \\
\text { dis- } \\
\text { solved } \\
\text { (mg/L } \\
\text { as K) }\end{array}$ \\
\hline $\begin{array}{l}01-31-84 \\
02-27-84 \\
03-28-84 \\
04-03-84 \\
04-25-84\end{array}$ & $\begin{array}{l}16: 00 \\
15: 10 \\
09: 00 \\
08: 45 \\
13: 00\end{array}$ & $\begin{array}{r}240 \\
-- \\
250 \\
-- \\
150\end{array}$ & $\begin{array}{l}8.1 \\
7.5 \\
7.7 \\
7.7 \\
7.5\end{array}$ & $\begin{array}{l}7.0 \\
7.5 \\
6.0 \\
7.0 \\
8.0\end{array}$ & $\begin{array}{r}82 \\
78 \\
100 \\
59 \\
59\end{array}$ & $\begin{array}{l}27 \\
26 \\
34 \\
20 \\
20\end{array}$ & $\begin{array}{l}3.5 \\
3.2 \\
4.0 \\
2.2 \\
2.1\end{array}$ & $\begin{array}{r}11 \\
6.5 \\
7.7 \\
3.1 \\
2.4\end{array}$ & $\begin{array}{r}10 \\
8.5 \\
9.3 \\
6.7 \\
6.8\end{array}$ \\
\hline $\begin{array}{l}04-25-84^{1} \\
06-06-84 \\
07-16-84 \\
09-12-84 \\
10-24-84\end{array}$ & $\begin{array}{l}13: 15 \\
09: 00 \\
13: 15 \\
09: 30 \\
14: 00\end{array}$ & $\begin{array}{l}-- \\
150 \\
193 \\
188 \\
219\end{array}$ & $\begin{array}{l}-- \\
7.5 \\
7.4 \\
7.6 \\
7.6\end{array}$ & $\begin{array}{l}-- \\
12.0 \\
12.5 \\
11.0 \\
10.0\end{array}$ & $\begin{array}{l}58 \\
55 \\
77 \\
71 \\
83\end{array}$ & $\begin{array}{l}20 \\
19 \\
27 \\
25 \\
29\end{array}$ & $\begin{array}{l}2.0 \\
1.8 \\
2.3 \\
2.1 \\
2.5\end{array}$ & $\begin{array}{l}2.5 \\
2.4 \\
2.7 \\
2.8 \\
3.3\end{array}$ & $\begin{array}{l}-- \\
7.7 \\
9.1 \\
10 \\
10\end{array}$ \\
\hline $\begin{array}{l}11-20-84 \\
12-20-84\end{array}$ & $\begin{array}{l}09: 15 \\
11: 30\end{array}$ & $\begin{array}{l}290 \\
263\end{array}$ & $\begin{array}{l}7.5 \\
7.4\end{array}$ & $\begin{array}{l}6.0 \\
8.5\end{array}$ & $\overline{87}$ & $\overline{31}$ & -- & -- & -- \\
\hline
\end{tabular}

See footnote at end of table.

\begin{tabular}{|c|c|c|c|c|c|c|c|c|c|c|}
\hline Date & $\begin{array}{l}\text { Nitro- } \\
\text { gen, } \\
\text { ammonia } \\
\text { total } \\
\text { (mg/L } \\
\text { as N) }\end{array}$ & $\begin{array}{l}\text { Nitro- } \\
\text { gen, } \\
\text { ammonia, } \\
\text { dis- } \\
\text { solved } \\
\text { (mg/L } \\
\text { as N) }\end{array}$ & $\begin{array}{c}\text { Alka- } \\
\text { linity } \\
\text { field } \\
\text { (mg/L } \\
\text { as } \\
\mathrm{CaCO}_{3} \text { ) }\end{array}$ & $\begin{array}{l}\text { Sulfate, } \\
\text { dis- } \\
\text { solved } \\
\text { (mg/L } \\
\text { as } \mathrm{SO}_{4} \text { ) }\end{array}$ & $\begin{array}{c}\text { Nitro- } \\
\text { gen, } \\
\text { nitrate, } \\
\text { dis- } \\
\text { solved } \\
(\mathrm{mg} / \mathrm{L} \\
\text { as } N)\end{array}$ & $\begin{array}{l}\text { Phos- } \\
\text { phorus, } \\
\text { ortho, } \\
\text { dis- } \\
\text { solved } \\
\text { (mg/L } \\
\text { as P) }\end{array}$ & $\begin{array}{l}\text { Chlo- } \\
\text { ride, } \\
\text { dis- } \\
\text { solved } \\
\text { (mg/L } \\
\text { as Cl) }\end{array}$ & $\begin{array}{l}\text { Fluo- } \\
\text { ride, } \\
\text { dis- } \\
\text { solved } \\
\text { (mg/L } \\
\text { as F) }\end{array}$ & $\begin{array}{l}\text { Silica, } \\
\text { dis- } \\
\text { solved } \\
\text { (mg/L } \\
\text { as } \\
\mathrm{SiO}_{2} \text { ) }\end{array}$ & $\begin{array}{l}\text { Alum- } \\
\text { inum, } \\
\text { dis- } \\
\text { solved } \\
(\mu \mathrm{g} / \mathrm{L} \\
\text { as Al) }\end{array}$ \\
\hline $01-31-84$ & -- & $<0.01$ & -- & 25 & -- & -- & 4.8 & 0.22 & 9.7 & 70 \\
\hline $02-27-84$ & $<0.001$ & .03 & -- & 16 & -- & -- & 2.4 & .22 & 10 & $<10$ \\
\hline $03-28-84$ & .036 & -- & 112 & 20 & -- & -- & 2.3 & $<.01$ & 11 & $<10$ \\
\hline $04-03-84$ & .103 & -- & 54 & 20 & -- & -- & 1.2 & .21 & 9.7 & 10 \\
\hline $04-25-84$ & .034 & -- & 52 & 21 & -- & -- & 1.2 & .17 & 9.9 & $<10$ \\
\hline $04-25-84$ & -- & -- & -- & -- & -- & -- & -- & -- & 19.7 & $1_{<10}$ \\
\hline $06-06-84$ & .064 & -- & 50 & 24 & -- & -- & 1.1 & .12 & 10 & - \\
\hline $07-16-84$ & .047 & -- & 65 & 29 & -- & -- & 1.5 & .13 & 10 & 10 \\
\hline $09-12-84$ & .122 & -- & 54 & 28 & -- & -- & 1.7 & .20 & 11 & 20 \\
\hline $10-24-84$ & -- & -- & 58 & 38 & $<0.01$ & $<0.01$ & 1.5 & .01 & 10 & 80 \\
\hline $11-20-84$ & .031 & -- & 64 & -- & -- & -- & -- & -- & -- & -- \\
\hline $12-20-84$ & .042 & -- & 51 & 120 & .01 & $<.01$ & .62 & .26 & 13 & $<10$ \\
\hline
\end{tabular}

See footnote at end of table. 
Table 15.--Physical characteristics and concentrations of chemical constituents of ground water at station number 422906072124301, Fever Brook till well near Petersham, Massachusetts--Continued

\begin{tabular}{|c|c|c|c|c|c|c|c|c|c|c|}
\hline Date & $\begin{array}{c}\text { Arsenic, } \\
\text { total } \\
\text { ( } \mu g / L \\
\text { as As) }\end{array}$ & $\begin{array}{l}\text { Copper, } \\
\text { dis- } \\
\text { solved } \\
(\mu g / L \\
\text { as Cu) }\end{array}$ & $\begin{array}{l}\text { Iron, } \\
\text { dis- } \\
\text { solved } \\
(\mu g / L \\
\text { as } \mathrm{Fe})\end{array}$ & $\begin{array}{c}\text { Lead, } \\
\text { dis- } \\
\text { solved } \\
(\mu g / L \\
\text { as } \mathrm{Pb})\end{array}$ & $\begin{array}{l}\text { Manga- } \\
\text { nese, } \\
\text { dis- } \\
\text { solved } \\
(\mu g / L \\
\text { as } M n)\end{array}$ & $\begin{array}{c}\text { Mercury, } \\
\text { dis- } \\
\text { solved } \\
\text { ( } \mu g / L \\
\text { as } \mathrm{Hg})\end{array}$ & $\begin{array}{l}\text { Sele- } \\
\text { nium, } \\
\text { dis- } \\
\text { solved } \\
\text { ( } \mu \text { g/L } \\
\text { as Se) }\end{array}$ & $\begin{array}{l}\text { Vana- } \\
\text { dium, } \\
\text { dis- } \\
\text { solved } \\
(\mu g / L \\
\text { as V) }\end{array}$ & $\begin{array}{c}\text { Carbon, } \\
\text { organic } \\
\text { total } \\
\text { (mg/L } \\
\text { as C) }\end{array}$ & $\begin{array}{l}\text { Carbon, } \\
\text { organic, } \\
\text { dis- } \\
\text { solved } \\
\text { (mg/L } \\
\text { as C) }\end{array}$ \\
\hline $01-31-84$ & -- & 0.9 & 18 & 1.5 & 320 & 0.2 & $<1$ & $<1$ & -- & -- \\
\hline $02-27-84$ & -- & 3.1 & 8 & 4.5 & 400 & .2 & $<1$ & $<1$ & 4.3 & -- \\
\hline $03-28-84$ & -- & .4 & 4 & .3 & 500 & .2 & $<1$ & $<1$ & 5.5 & -- \\
\hline $04-03-84$ & -- & 3.0 & 8 & .5 & 270 & $<.1$ & $<1$ & $<1$ & 1.5 & -- \\
\hline $04-25-84$ & $-\infty$ & 6.8 & 4 & 1.2 & 260 & $<.1$ & $<1$ & $<1$ & -- & 1.4 \\
\hline $04-25-84$ & -- & -- & $I_{33}$ & -- & $I_{250}$ & -- & -- & -- & -- & -- \\
\hline $06-06-84$ & -- & -- & 69 & -- & 270 & -- & -- & -- & -- & 1.6 \\
\hline $07-16-84$ & -- & 3.9 & 15 & .2 & 330 & $<.1$ & $<1$ & $<1$ & -- & 2.5 \\
\hline $09-12-84$ & $<1$ & 1.0 & 150 & .6 & 340 & $<.1$ & $<1$ & $<1$ & -- & 2.1 \\
\hline $10-24-84$ & -- & 1.0 & 97 & .9 & 350 & $<.1$ & $<1$ & 1 & -- & 2.1 \\
\hline $11-20-84$ & -- & -- & -- & -- & -- & -- & -- & -- & -- & -- \\
\hline $12-20-84$ & -- & .3 & 180 & 1.8 & 450 & -- & -- & -- & -- & -- \\
\hline
\end{tabular}

1 Sample filtered through 0.1 micrometer pore filter. 
Table 16.--Physical characteristics and concentrations of chemical constituents of ground water at station number 422910072120501, Knault's well near Petersham, Massachusetts

$\left[\mu \mathrm{S} / \mathrm{cm}\right.$, microsiemens per centimeter at 25 degrees Celsius; ${ }^{\circ} \mathrm{C}$, degrees Celsius; $\mathrm{mg} / \mathrm{L}$, milligrams per liter; $\mu \mathrm{g} / \mathrm{L}$, micrograms per liter, <, less than; dashes indicate not analyzed]

\begin{tabular}{|c|c|c|c|c|c|c|c|}
\hline Date & Time & $\begin{array}{l}\text { Spe- } \\
\text { cific } \\
\text { con- } \\
\text { duct- } \\
\text { ance } \\
(\mu \mathrm{s} / \mathrm{cm})\end{array}$ & $\begin{array}{c}\mathrm{pH} \\
\text { (stand- } \\
\text { ard } \\
\text { units) }\end{array}$ & $\begin{array}{c}\text { Temper- } \\
\text { ature } \\
\left({ }^{\circ} \mathrm{C}\right)\end{array}$ & $\begin{array}{l}\text { Hard- } \\
\text { ness } \\
(\mathrm{mg} / \mathrm{L} \\
\mathrm{as} \\
\left.\mathrm{CaCO}_{3}\right)\end{array}$ & $\begin{array}{c}\text { Calcium, } \\
\text { dis- } \\
\text { solved } \\
\text { (mg/L } \\
\text { as Ca) }\end{array}$ & $\begin{array}{l}\text { Magne- } \\
\text { sium, } \\
\text { dis- } \\
\text { solved } \\
\text { (mg/L } \\
\text { as Mg) }\end{array}$ \\
\hline $02-19-85$ & $12: 00$ & 106 & 7.2 & 7.5 & 26 & 5.5 & 3.0 \\
\hline $03-20-85$ & $14: 00$ & 102 & 7.5 & 7.5 & 25 & 5.4 & 2.9 \\
\hline $04-10-85$ & $10: 45$ & 102 & 7.3 & 7.0 & 26 & 5.4 & 3.0 \\
\hline $06-11-85$ & $11: 00$ & 99 & 6.6 & 10.0 & 27 & 5.9 & 3.1 \\
\hline $08-20-85$ & $11: 45$ & 102 & 7.1 & 11.0 & 26 & 5.5 & 3.0 \\
\hline $08-20-85^{1}$ & $11: 45$ & - & -- & -- & -- & -- & -- \\
\hline
\end{tabular}

\begin{tabular}{|c|c|c|c|c|c|c|c|c|}
\hline Date & $\begin{array}{l}\text { Sodium, } \\
\text { dis- } \\
\text { solved } \\
\text { (mg/L } \\
\text { as } \mathrm{Na} \text { ) }\end{array}$ & $\begin{array}{l}\text { Potas- } \\
\text { sium, } \\
\text { dis- } \\
\text { solved } \\
\text { (mg/L } \\
\text { as K) }\end{array}$ & $\begin{array}{c}\text { Nitro- } \\
\text { gen, } \\
\text { ammonia, } \\
\text { dis- } \\
\text { solved } \\
\text { (mg/L } \\
\text { as N) }\end{array}$ & $\begin{array}{c}\text { Alka- } \\
\text { linity } \\
\text { field } \\
\text { (mg/L } \\
\text { as } \\
\mathrm{CaCO}_{3} \text { ) }\end{array}$ & $\begin{array}{l}\text { Sulfate, } \\
\text { dis- } \\
\text { solved } \\
\text { (mg/L } \\
\text { as } \mathrm{SO}_{4} \text { ) }\end{array}$ & $\begin{array}{c}\text { Nitro- } \\
\text { gen, } \\
\text { nitrate, } \\
\text { dis- } \\
\text { solved } \\
\text { (mg/L } \\
\text { as } N \text { ) }\end{array}$ & $\begin{array}{c}\text { Nitro- } \\
\text { gen, } \\
\mathrm{NO}_{2}+\mathrm{NO}_{3} \\
\text { ais- } \\
\text { solved } \\
\text { (mg/L } \\
\text { as } \mathrm{N})\end{array}$ & $\begin{array}{l}\text { Phos- } \\
\text { phorus, } \\
\text { ortho, } \\
\text { dis- } \\
\text { solved } \\
\text { (mg/I } \\
\text { as P) }\end{array}$ \\
\hline $02-19-85$ & 8.6 & 5.1 & 0.04 & 39 & 3.6 & -- & $<0.10$ & 0.11 \\
\hline $03-20-85$ & 7.9 & 4.6 & $<.01$ & 41 & 3.7 & -- & $<.10$ & .11 \\
\hline $04-10-85$ & 8.2 & 4.6 & .03 & 41 & 4.0 & -- & $<.10$ & .09 \\
\hline $06-11-85$ & 7.7 & 4.7 & .03 & 41 & 4.8 & 0.05 & -- & $<.01$ \\
\hline $08-20-85$ & 7.3 & 4.4 & $<.01$ & 21 & 6.4 & .10 & -- & $<.01$ \\
\hline $08-20-85$ & $-\infty$ & -- & -- & -- & -- & -- & -- & -- \\
\hline
\end{tabular}

\begin{tabular}{|c|c|c|c|c|c|c|c|c|}
\hline Date & $\begin{array}{l}\text { Chlo- } \\
\text { ride, } \\
\text { dis- } \\
\text { solved } \\
\text { (mg/L } \\
\text { as Cl.) }\end{array}$ & $\begin{array}{l}\text { Fluo- } \\
\text { ride, } \\
\text { dis- } \\
\text { solved } \\
\text { (mg/L } \\
\text { as F) }\end{array}$ & $\begin{array}{l}\text { Silica, } \\
\text { dis- } \\
\text { solved } \\
\text { (mg/I } \\
\text { as } \\
\mathrm{SiO}_{2} \text { ) }\end{array}$ & $\begin{array}{l}\text { Alum- } \\
\text { inum, } \\
\text { dis- } \\
\text { solved } \\
(\mu \mathrm{g} / \mathrm{L} \\
\text { as Al) }\end{array}$ & $\begin{array}{l}\text { Gopper, } \\
\text { dis- } \\
\text { solved } \\
\text { ( } \mu g / L \\
\text { as Cu) }\end{array}$ & $\begin{array}{l}\text { Iron, } \\
\text { dis- } \\
\text { solved } \\
\text { ( } \mu \text { g/L } \\
\text { as Fe) }\end{array}$ & $\begin{array}{l}\text { Lead, } \\
\text { dis- } \\
\text { solved } \\
(\mu g / L \\
\text { as } \mathrm{Pb})\end{array}$ & $\begin{array}{l}\text { Manga- } \\
\text { nese, } \\
\text { dis- } \\
\text { solved } \\
(\mu g / L \\
\text { as } \mathrm{Mn})\end{array}$ \\
\hline $02-19-85$ & 4.5 & 0.70 & 21 & 50 & 0.6 & 330 & 0.1 & 400 \\
\hline $03-20-85$ & 3.6 & .60 & 23 & 30 & 2.0 & 19 & 2.0 & 410 \\
\hline $04-10-85$ & 3.1 & .60 & 24 & 30 & 5.4 & 120 & 2.1 & 420 \\
\hline $06-11-85$ & 2.2 & .75 & 25 & 40 & 2.0 & 150 & 1.0 & 420 \\
\hline $08-20-85$ & 1.9 & .76 & 25 & - & 6.0 & 47 & 3.0 & 420 \\
\hline $08-20-85$ & -- & -- & -- & $1_{40}$ & -- & -- & -- & -- \\
\hline
\end{tabular}

1 Sample filtered through 0.1 micrometer pore filter. 
Table 17.--Physical characteristics and concentrations of chemical constituents of ground water at station number 422906072130201, Fever Brook sand and gravel well near Petersham, Massachusetts

$\left[\mu \mathrm{S} / \mathrm{cm}\right.$, microsiemens per centimeter at 25 degrees Celsius; ${ }^{\circ} \mathrm{C}$, degrees Celsius; $\mathrm{mg} / \mathrm{L}$, milligrams per liter;

$\mu \mathrm{g} /$, micrograms per liter, <, less than; dashes indicate not analyzed]

\begin{tabular}{|c|c|c|c|c|c|c|c|c|c|}
\hline $\begin{array}{l}12-20-83 \\
01-30-84 \\
02-27-84 \\
03-28-84 \\
04-25-84\end{array}$ & $\begin{array}{l}11: 45 \\
15: 30 \\
16: 45 \\
10: 30 \\
14: 00\end{array}$ & $\begin{array}{l}63 \\
57 \\
-- \\
54 \\
60\end{array}$ & $\begin{array}{l}5.9 \\
6.3 \\
6.2 \\
6.1 \\
6.2\end{array}$ & $\begin{array}{l}7.5 \\
7.5 \\
7.5 \\
7.0 \\
7.0\end{array}$ & $\begin{array}{l}13 \\
12 \\
13 \\
12 \\
12\end{array}$ & $\begin{array}{l}3.6 \\
3.4 \\
3.5 \\
3.4 \\
3.4\end{array}$ & $\begin{array}{l}1.0 \\
.93 \\
.92 \\
.89 \\
.89\end{array}$ & $\begin{array}{l}3.4 \\
3.2 \\
3.3 \\
3.1 \\
3.0\end{array}$ & $\begin{array}{r}0.94 \\
.85 \\
.85 \\
.82 \\
.79\end{array}$ \\
\hline $\begin{array}{l}04-25-84^{1} \\
06-06-84 \\
07-16-84 \\
09-12-84 \\
10-24-84\end{array}$ & $\begin{array}{l}14: 15 \\
11: 00 \\
14: 30 \\
11: 00 \\
15: 30\end{array}$ & $\begin{array}{l}-- \\
60 \\
64 \\
63 \\
64\end{array}$ & $\begin{array}{l}-- \\
6.1 \\
5.9 \\
6.2 \\
6.1\end{array}$ & $\begin{array}{l}-- \\
-- \\
10.5 \\
9.0 \\
9.5\end{array}$ & $\begin{array}{l}12 \\
12 \\
12 \\
12 \\
12\end{array}$ & $\begin{array}{l}3.4 \\
3.4 \\
3.4 \\
3.3 \\
3.4\end{array}$ & $\begin{array}{l}.90 \\
.89 \\
.94 \\
.89 \\
.90\end{array}$ & $\begin{array}{l}3.0 \\
3.0 \\
3.0 \\
3.1 \\
3.4\end{array}$ & $\begin{array}{r}-- \\
.81 \\
.86 \\
.98 \\
.86\end{array}$ \\
\hline $\begin{array}{l}04-10-85 \\
06-11-85 \\
08-20-85\end{array}$ & $\begin{array}{l}09: 00 \\
10: 00 \\
10: 15\end{array}$ & $\begin{array}{l}62 \\
61 \\
66\end{array}$ & $\begin{array}{l}6.1 \\
5.4 \\
5.9\end{array}$ & $\begin{array}{l}6.5 \\
8.0 \\
9.0\end{array}$ & $\begin{array}{l}12 \\
13 \\
13\end{array}$ & $\begin{array}{l}3.3 \\
3.6 \\
3.5\end{array}$ & $\begin{array}{l}.87 \\
.96 \\
.94\end{array}$ & $\begin{array}{l}2.9 \\
3.0 \\
3.2\end{array}$ & $\begin{array}{l}.79 \\
.80 \\
.84\end{array}$ \\
\hline
\end{tabular}

See footnote at end of table.

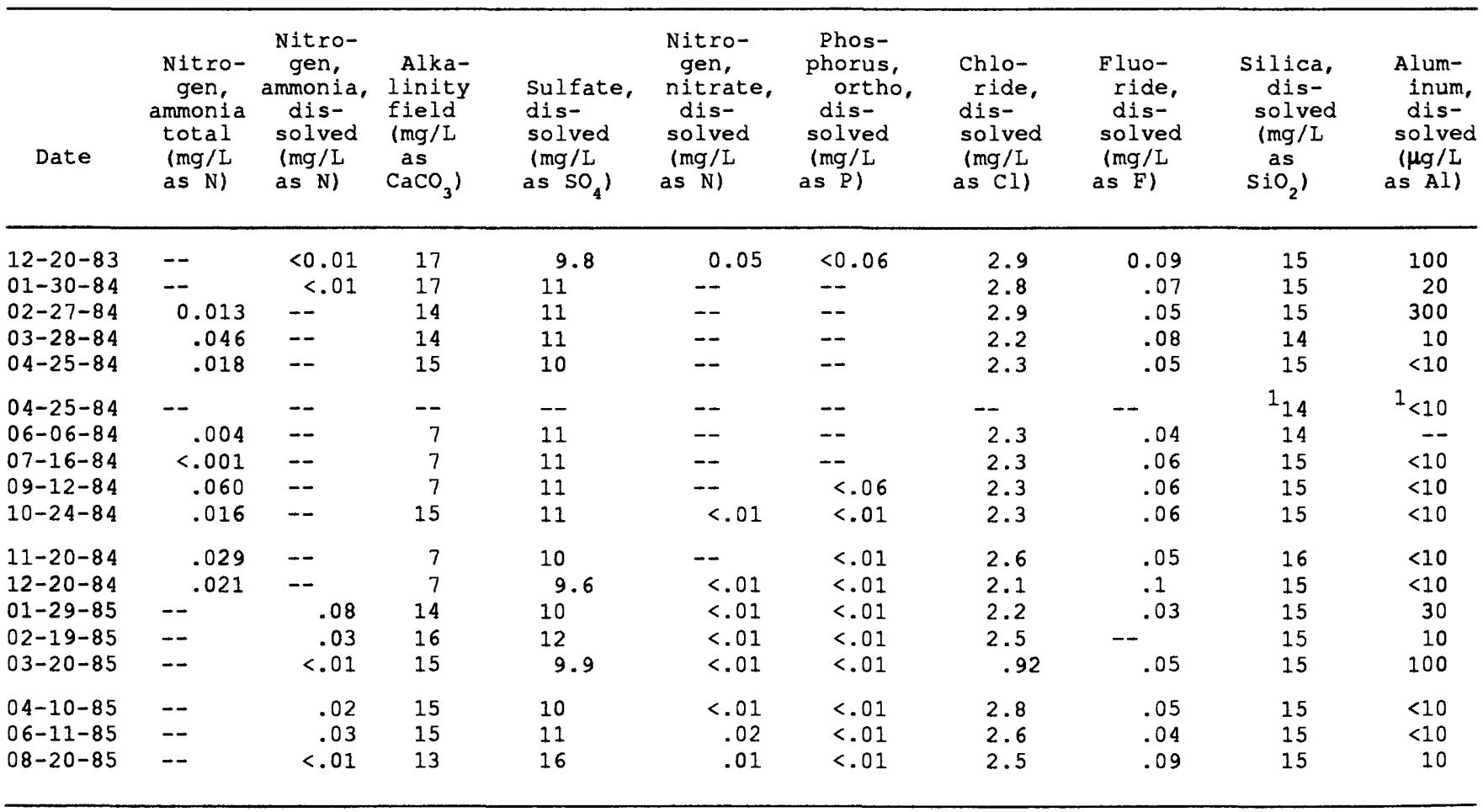

See footnote at end of table. 
Table 17.--Physical characteristics and concentrations of chemical constituents of ground water at station number 422906072130201, Fever Brook sand and gravel well near Petersham, Massachusetts--Continued

\begin{tabular}{|c|c|c|c|c|c|c|c|c|c|c|}
\hline Date & $\begin{array}{c}\text { Arsenic, } \\
\text { total } \\
(\mu \mathrm{g} / \mathrm{L} \\
\text { as As) }\end{array}$ & $\begin{array}{c}\text { Copper, } \\
\text { dis- } \\
\text { solved } \\
\text { ( } \mu \mathrm{g} / \mathrm{L} \\
\text { as } \mathrm{Cu})\end{array}$ & $\begin{array}{l}\text { Iron, } \\
\text { dis- } \\
\text { solved } \\
(\mu g / L \\
\text { as } \mathrm{Fe})\end{array}$ & $\begin{array}{l}\text { Lead, } \\
\text { dis- } \\
\text { solved } \\
(\mu g / L \\
\text { as } \mathrm{Pb})\end{array}$ & $\begin{array}{l}\text { Manga- } \\
\text { nese, } \\
\text { dis- } \\
\text { solved } \\
\text { ( } \mu \text { g/L } \\
\text { as } M n)\end{array}$ & $\begin{array}{c}\text { Mercury, } \\
\text { dis- } \\
\text { solved } \\
\text { ( } \mu g / L \\
\text { as } \mathrm{Hg})\end{array}$ & $\begin{array}{l}\text { Sele- } \\
\text { nium, } \\
\text { dis- } \\
\text { solved } \\
(\mu \mathrm{g} / \mathrm{L} \\
\text { as Se) }\end{array}$ & $\begin{array}{l}\text { Vana- } \\
\text { dium, } \\
\text { dis- } \\
\text { solved } \\
(\mu \mathrm{g} / \mathrm{L} \\
\text { as V) }\end{array}$ & $\begin{array}{c}\text { Carbon, } \\
\text { organic } \\
\text { total } \\
\text { (mg/L } \\
\text { as C) }\end{array}$ & $\begin{array}{l}\text { Carbon, } \\
\text { organic, } \\
\text { dis- } \\
\text { solved } \\
\text { (mg/L } \\
\text { as C) }\end{array}$ \\
\hline $\begin{array}{l}12-20-83 \\
01-30-84 \\
02-27-84 \\
03-28-84 \\
04-25-84\end{array}$ & $\begin{array}{l}-- \\
-- \\
-- \\
-- \\
--\end{array}$ & $\begin{array}{r}0.1 \\
.4 \\
1.8 \\
1.7 \\
4.4\end{array}$ & $\begin{array}{l}5600 \\
5500 \\
5600 \\
5500 \\
5500\end{array}$ & $\begin{array}{r}0.1 \\
1.5 \\
.8 \\
2.5 \\
1.5\end{array}$ & $\begin{array}{l}65 \\
62 \\
63 \\
62 \\
64\end{array}$ & $\begin{array}{l}<0.1 \\
<.1 \\
<.1 \\
<.1 \\
<.1\end{array}$ & $\begin{array}{l}<1 \\
<1 \\
<1 \\
<1 \\
<1\end{array}$ & $\begin{array}{r}<1 \\
<1 \\
<1 \\
1 \\
<1\end{array}$ & $\begin{array}{c}-- \\
-\overline{1.3} \\
.9\end{array}$ & $\begin{array}{r}1.1 \\
2.9 \\
-- \\
-- \\
.8\end{array}$ \\
\hline $\begin{array}{l}04-25-84 \\
06-06-84 \\
07-16-84 \\
09-12-84 \\
10-24-84\end{array}$ & $\begin{array}{l}-- \\
-- \\
-- \\
<1 \\
--\end{array}$ & $\begin{array}{l}-- \\
-- \\
1.1 \\
2.0 \\
.2\end{array}$ & $\begin{array}{r}1_{5200} \\
5400 \\
5400 \\
5100 \\
5300\end{array}$ & $\begin{array}{r}-- \\
-- \\
1.0 \\
.7 \\
.4\end{array}$ & $\begin{array}{r}{ }^{1} 67 \\
62 \\
64 \\
62 \\
61\end{array}$ & $\begin{array}{l}- \\
<.1 \\
<.1 \\
<.1\end{array}$ & $\begin{array}{l}-- \\
-- \\
<1 \\
<1 \\
<1\end{array}$ & $\begin{array}{r}-- \\
-- \\
<1 \\
1 \\
<1\end{array}$ & $\begin{array}{l}-- \\
-- \\
-- \\
-- \\
--\end{array}$ & $\begin{array}{r}-7 \\
1.2 \\
.6 \\
.3\end{array}$ \\
\hline $\begin{array}{l}11-20-84 \\
12-20-84 \\
01-29-85 \\
02-19-85 \\
03-20-85\end{array}$ & $\begin{array}{l}<1 \\
-- \\
-- \\
-- \\
--\end{array}$ & $\begin{array}{r}.2 \\
.6 \\
1.2 \\
.5 \\
<.1\end{array}$ & $\begin{array}{l}5200 \\
5200 \\
5100 \\
5300 \\
5100\end{array}$ & $\begin{array}{r}.1 \\
1.4 \\
.1 \\
.1 \\
2.0\end{array}$ & $\begin{array}{l}62 \\
61 \\
61 \\
61 \\
60\end{array}$ & $\begin{array}{l}-- \\
-- \\
-- \\
-- \\
--\end{array}$ & $\begin{array}{l}<1 \\
-- \\
-- \\
-- \\
--\end{array}$ & $\begin{array}{l}<1 \\
-- \\
-- \\
-- \\
--\end{array}$ & $\begin{array}{l}-- \\
-- \\
-- \\
-- \\
--\end{array}$ & $\begin{array}{l}.8 \\
-- \\
-- \\
--\end{array}$ \\
\hline $\begin{array}{l}04-10-85 \\
06-11-85 \\
08-20-85\end{array}$ & $\begin{array}{l}-- \\
-- \\
--\end{array}$ & $\begin{array}{l}.1 \\
<.1 \\
3.0\end{array}$ & $\begin{array}{l}5000 \\
5200 \\
5200\end{array}$ & $\begin{array}{l}.1 \\
<.1 \\
1.0\end{array}$ & $\begin{array}{l}59 \\
60 \\
60\end{array}$ & $\begin{array}{l}-- \\
-- \\
--\end{array}$ & $\begin{array}{l}-- \\
-- \\
--\end{array}$ & $\begin{array}{l}-- \\
-- \\
--\end{array}$ & $\begin{array}{l}-- \\
-- \\
--\end{array}$ & $\begin{array}{l}-- \\
-- \\
--\end{array}$ \\
\hline
\end{tabular}

1 sample filtered through 0.1 micrometer pore filter. 
Table 18.--Description of soil-collection sites and samplings

[See figs. 1 and 2 for sampling locations]

\begin{tabular}{|c|c|c|c|c|}
\hline $\begin{array}{l}\text { Site } \\
\text { desig- } \\
\text { nation }\end{array}$ & $\begin{array}{c}\text { Site } \\
\text { description }\end{array}$ & $\begin{array}{l}\text { Sample } \\
\text { desig- } \\
\text { nation }\end{array}$ & $\begin{array}{l}\text { Depth from } \\
\text { surface, } \\
\text { (1n feet) }\end{array}$ & Sample description \\
\hline
\end{tabular}

\section{SWIFT RIVER BASIN}

SSI

T1ll-covered slopes; samples collected in topographic low, just before break in slope. Hardwood stands, estimated helghts 50 to 60 feet; moderate understory (ferns,

laurels, spruce).

West-facing moderate slope; hardwoods and moderate understory. slopes; mix of conifer and hardwood stands.

Fresh face of plt; mix of hardwoods and contfers on unbroken ground. Sampled at top of 50 feet of exposed sequence of sands and gravels.

SS4A-1 SS $4 A-2$ SS $4 A-3$

ss $3-2$

$$
\begin{aligned}
& 0.0 \text { to } 0.5 \\
& 0.5 \text { to } 0.85 \\
& 0.85 \text { to } 1.00 \\
& 1.00 \text { to } 1.25 \\
& 1.25 \text { to } 2.0 \\
& 2.0 \text { to } 2.3
\end{aligned}
$$

0.0 to 0.2 0.02 to 1.4 1.4 to 2.1 2.1 to 2.85

$$
0.0 \text { to } 0.2
$$$$
0.2 \text { to } 0.5
$$

$$
0.5 \text { to } 0.8
$$

0.0 to 0.3
0.3 to 1.7
1.7 to 1.9
0.9 to 1.7
1.7 to 1.85

\section{FEVER BROOK BASIN}

$\begin{array}{ll}\text { FS1 } & \begin{array}{c}\text { Moderate to gent le gra- } \\ \text { dients, till slopes; } \\ \text { heavy understory, young } \\ \text { and mature conifer stands. }\end{array} \\ \text { FS2 } & \text { Gentle gradient, some kettle } \\ & \text { topography; heavy leaf } \\ & \text { litter, light understory } \\ & \text { growth. } \\ & \text { Moderate growth of hardwoods } \\ & \text { and conifers, moderate } \\ & \text { fern understory; gentle } \\ & \text { gradients. } \\ & \text { Near swampy areas; conifer } \\ & \text { stands, light to moderate } \\ & \text { understory growth; gentle- } \\ & \text { to-flat gradients. }\end{array}$

FSI-1

FSI-2

FSI-3

ES2-1

FS2-2

ES2-3

ES3-1

ES3-2

ES3-3

F S4-I

FS4-2
0.0 to 0.2

0.2 to 2.2

2.2 to 3.1

0.0 to 0.5

0.5 to 0.8

0.8 to 2.3

0.0 to 0.4

0.4 to 1.0

1.0 to 2.2

0.0 to 0.6

0.6 to 1.6
Medium to dark brown, silty, micaceous, roots. Medium to light brown, silty. Medium to dark brown, dense, clayey. Brown to black, rootlets, loose.

Dark brown, fine sand, some rootlets. Medium brown, silty to sandy, some coarse sand and pebbles.

Brown to black, organic, rootlets. Medium to dark brown, rootlets, clayey.

Medium brownish-red, rootlets, silty and sandy. Medium brown, slight yellow, silty

to fine sandy, black specks.

Leaf litter, no sample.

Dark brown, rootlets, loamy, some pebbles Brown, reddish, pebbly, loose, silty.

Brown, loamy, rootlets.

Brown, yellowlsh-red, loamy. Lyyic brown, silty, pebbles.

Brown, reddish, loamy. Brown, yellowish, silt and fine sand.
Dark brown, loamy, molst, rootlets. Brown, red-stalned; loamy to slity. Brown, yellowish, silty to fine sandy.

Black, organic, rootlets. Reddish, brown, I inch dense clayey zone, rootlets. Brown, yellow stains, dense.

Black, organic-rich, rootlets. Brown, red-stained, clayey. Brown, red and yellow stained, pebbly; some fresh surfaces grey.

Black, organic, loose, rootlets. Brown, reddish-stalned, loose, loamy.

$1 \mathrm{~A}$ and $\mathrm{B}$ designations for two separately driven holes. 


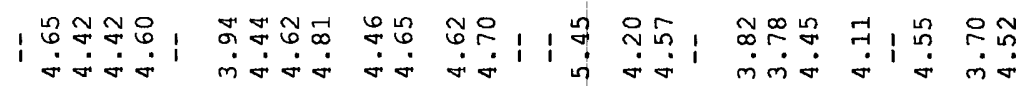

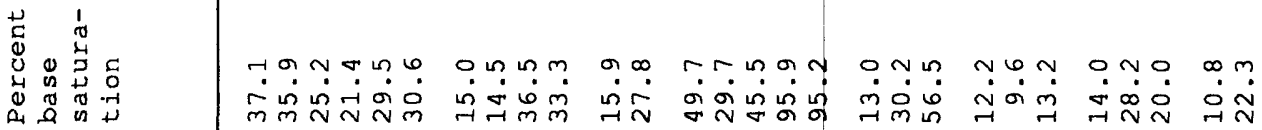

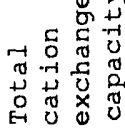

mơr $m$

نำ

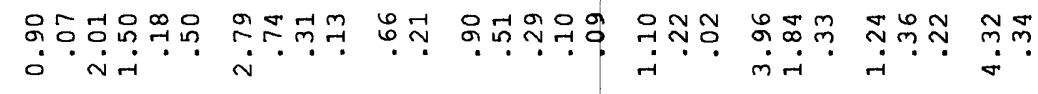

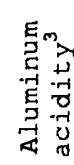

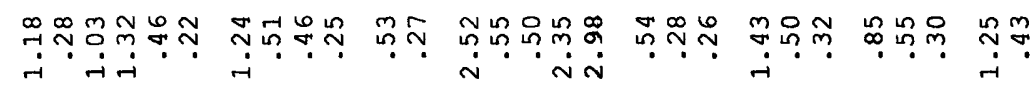

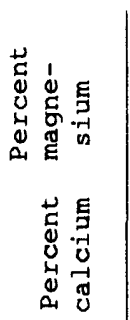

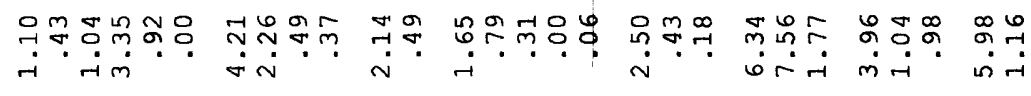
空 !

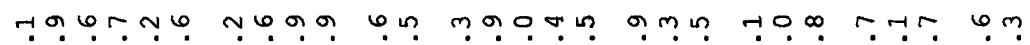
ஸेન્ન

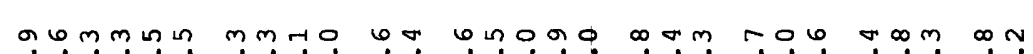
ஸ่

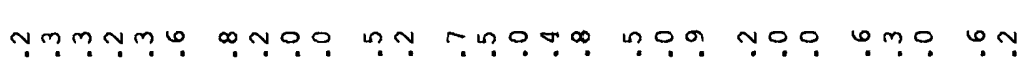

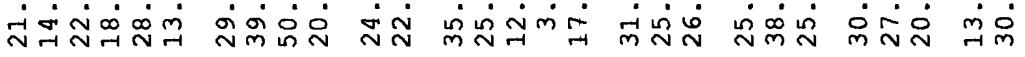
范

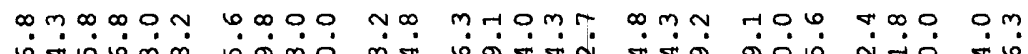

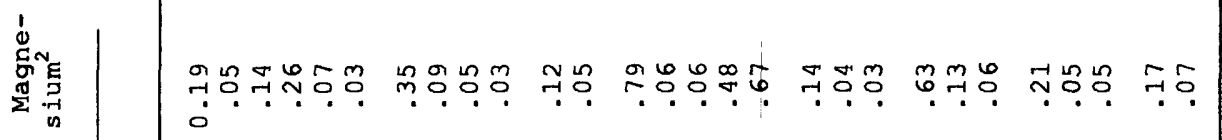
岂㺃

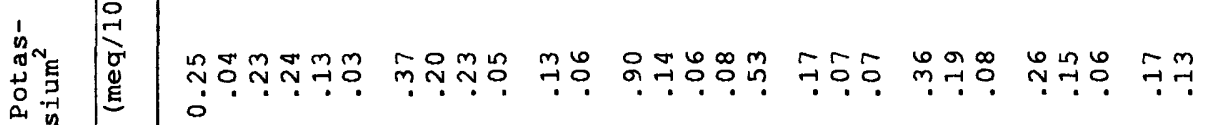

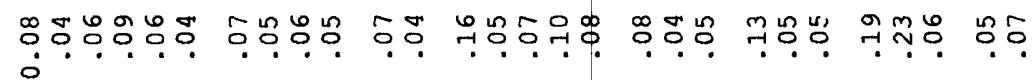
晋 
Table 20.--Results of mineralogic analysis of clay-size (less than 2 micrometers) fraction of soil samples

[M, major mineral component; $m$, minor mineral component; $t$, trace mineral component; ?, possible, difficult to ascertain from $X$-ray diffraction pattem. Interlayer (additional clay material between main structural layers): Lower case letter, minor component of total clay mineralogy, upper case letter, major component of total clay mineralogy; V, v = vermiculite; $C, c=$ chlorite; $I, i=$ illite]

\begin{tabular}{|c|c|c|c|c|c|c|c|}
\hline Sample ${ }^{1}$ & $\begin{array}{l}\text { Vermic- } \\
\text { ulite }\end{array}$ & Illite & $\begin{array}{l}\text { Chlo- } \\
\text { rite }\end{array}$ & $\begin{array}{l}\text { Gibb- } \\
\text { site }\end{array}$ & $\begin{array}{l}\text { Kaolin- } \\
\text { ite }\end{array}$ & Interlayer & Remarks \\
\hline ss $1-1$ & $\mathbf{M}$ & m & -- & $\mathrm{m}$ & $\mathrm{m}$ & $v-i$ & \\
\hline ss1-2 & $\mathbf{M}$ & $\mathbf{m}$ & -- & m & $\mathrm{m}$ & $v-i$ & \\
\hline SS $1-5$ & M & $\mathrm{m}$ & $\mathrm{m}$ & $\mathrm{m}$ & $m$ & $v-i ; i-c$ & \\
\hline SS $1-6$ & $\mathbf{M}$ & $\mathrm{m}$ & $\mathrm{m}$ & -- & $?$ & $\mathrm{~V}-\mathrm{I} ; \mathrm{V}-\mathrm{C}$ & \\
\hline ss2-2 & $M$ & $\mathrm{~m}$ & -- & $t$ & $\mathrm{~m}$ & $v-1 ; v-c ?$ & \\
\hline ss $2-3$ & $\mathbf{M}$ & m & $t ?$ & $\mathbf{M}$ & -- & $v-1 ; v-c ; c-1 ?$ & \\
\hline ss $2-4$ & $\mathbf{M}$ & $\mathrm{m}$ & -- & $\mathbf{M}$ & $\mathrm{m}$ & $V-I$ & \\
\hline ss $3-2$ & $\mathbf{M}$ & $\mathrm{m}$ & -- & $\mathbf{M}$ & -- & $c-i ?$ & $\begin{array}{l}\text { Very low intensities } \\
\text { of all peaks }\end{array}$ \\
\hline SS 4-1 & $\mathbf{M}$ & $\mathrm{m}$ & -- & -- & m & $c-i ; v-i ?$ & \\
\hline SS4-2 & $\mathbf{M}$ & $\mathrm{m}$ & $\mathrm{m}$ & $\mathrm{m}$ & $\mathrm{m}$ & $v-c$ & \\
\hline SS 4-1B & $\mathbf{M}$ & $\mathrm{m}$ & $M$ & $\mathrm{~m}$ & $t ?$ & $v-c$ & \\
\hline$S S 4-2 B$ & $\mathbf{M}$ & $\mathbf{M}$ & $\mathbf{M}$ & -- & $\mathrm{m} ?$ & $v-c$ & \\
\hline FS1-2 & $\mathbf{M}$ & m & $\mathbf{M}$ & $\mathrm{m}$ & $\mathrm{m}$ & $v-C ; v-i$ & \\
\hline FS1-3 & M & $\mathbf{M}$ & $\mathbf{M}$ & -- & $\mathrm{m}$ & $v-1$ & \\
\hline FS2-3 & $\mathbf{M}$ & $\mathrm{m}$ & $\mathbf{M}$ & -- & -- & $v-c ; c-1$ & \\
\hline FS3-2 & $\mathbf{M}$ & m & -- & -- & $\mathrm{m}$ & $v-c ; v-i$ & \\
\hline FS3-3 & $\mathbf{M}$ & $\mathbf{M}$ & $\mathrm{m}$ & -- & $\mathrm{m}$ & $V-I ; v-c$ & \\
\hline FS4-2 & & $m$ & $m$ & -- & $\mathrm{m}$ & $v-c ; v-i$ or $c-i$ & $\begin{array}{l}\text { Low intensities of } \\
\text { all peaks except } \\
14 \text { Angstrom }\end{array}$ \\
\hline
\end{tabular}

1 Refer to table 18 for sample sites and descriptions. 
Table 21.--Physical characteristics and concentrations of chemical constituents of soil waters from the West Branch Swift River and East Branch Fever Brook basins

$\left[\mu \mathrm{S} / \mathrm{cm}\right.$, microsiemens per centimeter; ${ }^{\circ} \mathrm{C}$, degrees Celsius; $\mathrm{mg} / \mathrm{L}$, milligrams per liter, $\mu \mathrm{g} / \mathrm{L}$, micrograms per liter, $<$, less than; dashes indicate not analyzed]

\begin{tabular}{|c|c|c|c|c|c|c|c|c|c|}
\hline Date & $\begin{array}{l}\text { Spe- } \\
\text { cific } \\
\text { con- } \\
\text { duct- } \\
\text { ance } \\
(\mu s / \mathrm{cm})\end{array}$ & $\begin{array}{c}\mathrm{pH} \\
\text { (stand- } \\
\text { ard } \\
\text { units) }\end{array}$ & $\begin{array}{l}\text { Temper- } \\
\text { ature } \\
.\left({ }^{\circ} \mathrm{C}\right)\end{array}$ & $\begin{array}{l}\text { Hard- } \\
\text { ness } \\
\text { (mg/L } \\
\text { as } \\
\text { CaCO) }\end{array}$ & $\begin{array}{l}\text { Calcium, } \\
\text { dis- } \\
\text { solved } \\
\text { (mg/I } \\
\text { as Ca) }\end{array}$ & $\begin{array}{l}\text { Magne- } \\
\text { sium, } \\
\text { dis- } \\
\text { solved } \\
\text { (mg/L } \\
\text { as Mg) }\end{array}$ & $\begin{array}{l}\text { Sodium, } \\
\text { dis- } \\
\text { solved } \\
\text { (mg/L } \\
\text { as Na) }\end{array}$ & $\begin{array}{l}\text { Potas- } \\
\text { sium, } \\
\text { dis- } \\
\text { solved } \\
\text { (mg/L } \\
\text { as K) }\end{array}$ & $\begin{array}{l}\text { Nitro- } \\
\text { gen, } \\
\text { ammonia } \\
\text { total } \\
\text { (mg/L } \\
\text { as N) }\end{array}$ \\
\hline
\end{tabular}

Swift River basin soil-water sampler at station number 422800072232801

$\begin{array}{rr}10-15-84 & 76 \\ 10-24-84 & 47 \\ 03-11-85 & -- \\ 07-31-85 & -- \\ 08-15-85 & 77 \\ 08-22-85 & 126\end{array}$

$05-30-85$

$08-02-85$

08-20-85

04-07-84

03-05-85

03-26-85

03-01-84

$03-21-84$

04-07-84

$04-18-84$

04-19-84

05-29-85

06-28-85

08-02-85

08-08-85

$08-20-85$

$05-30-85$

$08-02-85$

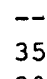

39

\section{1}

46

$-1$

$\begin{array}{llr}6.1 & -- & -- \\ 5.6 & -- & 9 \\ -- & -- & 4 \\ 5.5 & 20.0 & -- \\ 5.8 & 19.0 & 15 \\ 5.2 & -- & --\end{array}$

Swift River basin deep lysimeter at station number 422800072233101

$\begin{array}{lll}5.4 & -- & 19.0 \\ 5.3 & 21.0 & -\end{array}$

Fox Den, Fever Brook basin at station number 422848072131701

\begin{tabular}{llcc|ccc}
5.3 & -- & 7 & 2.4 & .36 & .65 & 17 \\
5.4 & -- & -- & -- & -- & -- & -1 \\
7.0 & -- & 11 & 3.3 & .57 & .5 & 2.8
\end{tabular}

0.171

$-$

Fever Brook basin soil-water sampler at station number 422901072121201

$\begin{array}{rrrrrrr}4.8 & -- & 2 & .41 & .24 & 1.2 & .10 \\ -- & -- & -- & -- & -- & -. & .09 \\ 5.0 & -- & 2 & .42 & .22 & 1.3 & .33 \\ 5.5 & -- & 2 & .48 & .22 & 1.3 & -.\end{array}$

Fever Brook basin deep lysimeter at station number 422909072120901

$\begin{array}{rrrrrrrr}5.0 & -- & 7 & 1.7 & .68 & 3.6 & .40 & -- \\ 4.8 & 13.0 & 8 & 2.1 & .76 & 4.2 & .48 \\ 4.8 & 16.0 & 8 & 1.9 & .77 & 3.8 & -- \\ 5.0 & 16.5 & -- & -- & -- & -- & -- \\ 4.9 & 16.5 & -- & -- & -- & -- & --\end{array}$

Fever Brook basin medium lysimeter at station number 422909072121001

$\begin{array}{llll}4.9 & -- & 7 & 1.7 \\ 4.6 & 17.0 & 8 & 2.0\end{array}$

1.7
2.0

.66

3.6

4.4

.47

.54 
Table 21.--Physical characteristics and concentrations of chemical constituents of soil waters from the West Branch Swift River and East Branch Fever Brook basins--Continued

\begin{tabular}{|c|c|c|c|c|c|c|c|}
\hline Date & $\begin{array}{l}\text { Nitro- } \\
\text { gen, } \\
\text { ammonia, } \\
\text { dis- } \\
\text { solved } \\
\text { (mg/L } \\
\text { as N) }\end{array}$ & $\begin{array}{c}\text { Alka- } \\
\text { linity } \\
\text { field } \\
\text { (mg/L } \\
\text { as } \\
\mathrm{CaCO}_{3} \text { ) }\end{array}$ & $\begin{array}{l}\text { Sulfate, } \\
\text { dis- } \\
\text { solved } \\
\text { (mg/L } \\
\text { as } \mathrm{SO}_{4} \text { ) }\end{array}$ & $\begin{array}{c}\text { Nitro- } \\
\text { gen, } \\
\text { nitrate, } \\
\text { dis- } \\
\text { solved } \\
\text { (mg/L } \\
\text { as N) }\end{array}$ & $\begin{array}{l}\text { Phos- } \\
\text { phorus, } \\
\text { ortho, } \\
\text { dis- } \\
\text { solved } \\
\text { (mg/L } \\
\text { as P) }\end{array}$ & $\begin{array}{l}\text { Chlo- } \\
\text { ride, } \\
\text { dis- } \\
\text { solved } \\
\text { (mg/L } \\
\text { as Cl) }\end{array}$ & $\begin{array}{l}\text { Fluo- } \\
\text { ride, } \\
\text { dis- } \\
\text { solved } \\
\text { (mg/L } \\
\text { as F) }\end{array}$ \\
\hline
\end{tabular}

Swift River basin soil-water sampler at station number 422800072232801

$10-15-84$

$10-24-84$

$03-11-85$

$07-31-85$

08-15-85

$08-22-85$

$05-30-85$

$08-02-85$

$08-20-85$

$04-07-84$

03-05-85

$03-26-85$

03-01-84

$03-21-84$

04-07-84

04-18-84

$04-19-84$

$05-29-85$

$06-28-85$

08-02-85

08-08-85

$08-20-85$

$05-30-85$

08-02-85

$\begin{array}{rrr}-- & 6 & -- \\ -- & 2 & 9.3 \\ 0.49 & -- & 1.7 \\ .62 & -- & 10 \\ -57 & -- & 16 \\ -. & -- & --\end{array}$

--
2.01
2.60

--
$<0.01$
$<.01$

$--1$

.2

$--$

$-$

$-$

$<0.01$

.10

$<.01$

2.1

.10

Swift River basin deep lysimeter at station number 422800072233101

$\begin{array}{ccccccc}-- & -- & - & -- & -- & \\ 0.05 & -- & 9.4 & 0.03 & <0.01 & -- & 0.13 \\ -- & -- & -- & -- & --\end{array}$

Fox Den, Fever Brook basin at station number 422848072131701

$\begin{array}{ccccccr}0.19 & -- & 12 & 0.38 & <0.06 & 2.6 & <0.01 \\ - & -- & -- & -- & -- & -- & -0 \\ 4.20 & -- & 7.9 & 1.70 & .18 & .74 & .04\end{array}$

Fever Brook basin soil-water sampler at station number 422901072121201

\begin{tabular}{|c|c|c|c|c|c|c|}
\hline-- & -- & 7.7 & -- & -- & 2.1 & 0.06 \\
\hline-- & -- & -- & -- & -- & -- & -- \\
\hline-- & -- & 7.6 & -- & -- & 1.6 & .04 \\
\hline-- & -- & 7.8 & -- & -- & 1.5 & .05 \\
\hline -- & -- & -- & -- & -- & -- & -- \\
\hline
\end{tabular}

Fever Brook basin deep lysimeter at station number 422909072120901

$\begin{array}{rrrrrrr}0.07 & -- & 8.1 & <0.01 & <0.01 & 7.8 & 0.28 \\ <.01 & -- & 6.4 & .01 & <.01 & 10 & .09 \\ .02 & -- & 5.8 & -02 & -.01 & 7.0 & .04 \\ -- & -- & -- & -- & -- & -- & --\end{array}$

Fever Brook basin medium lysimeter at station number 422909072121001

$\begin{array}{rrrrrrr}0.03 & -- & 7.8 & <0.01 & <0.01 & 8.1 & 0.13 \\ .04 & -- & 5.7 & .02 & <.01 & 9.4 & .10\end{array}$


Table 21.--Physical characteristics and concentrations of chemical constituents of soil waters from the West Branch Swift River and East Branch Fever Brook basins--Continued

\begin{tabular}{|c|c|c|c|c|c|c|}
\hline Date & $\begin{array}{c}\text { Silica, } \\
\text { dis- } \\
\text { solved } \\
\text { (mg/L } \\
\mathrm{as} \\
\mathrm{SiO}_{2} \text { ) }\end{array}$ & $\begin{array}{l}\text { Alum- } \\
\text { inum, } \\
\text { dis- } \\
\text { solved } \\
(\mu g / L \\
\text { as Al) }\end{array}$ & $\begin{array}{c}\text { Arsenic, } \\
\text { total } \\
\text { ( } \mu \text { go/ } \\
\text { as As) }\end{array}$ & $\begin{array}{l}\text { Copper, } \\
\text { dis- } \\
\text { solved } \\
(\mu g / L \\
\text { as Cu) }\end{array}$ & $\begin{array}{l}\text { Iron, } \\
\text { dis- } \\
\text { solved } \\
\text { ( } \mu \text { g/L } \\
\text { as Fe) }\end{array}$ & $\begin{array}{l}\text { Lead, } \\
\text { dis- } \\
\text { solved } \\
(\mu g / L \\
\text { as } \mathrm{Pb})\end{array}$ \\
\hline
\end{tabular}

Swift River basin soil-water sampler at station number 422800072232801

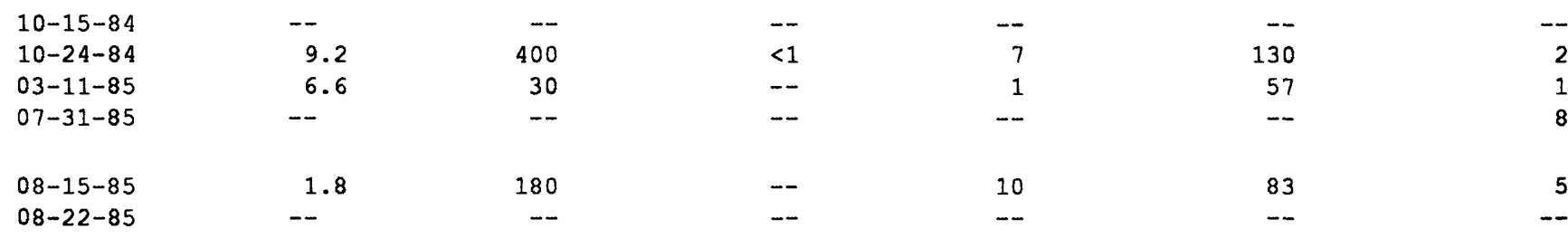

Swift River basin deep lysimeter at station number 422800072233101

$05-30-85$

$08-02-85$

$08-20-85$

$04-07-84$

$03-05-85$

$03-26-85$

03-01-84

$03-21-84$

$04-07-84$

$04-18-84$

$04-19-84$

$05-29-85$

$06-28-85$

$08-02-85$

$08-08-85$

$08-20-85$

$05-30-85$

$08-02-85$

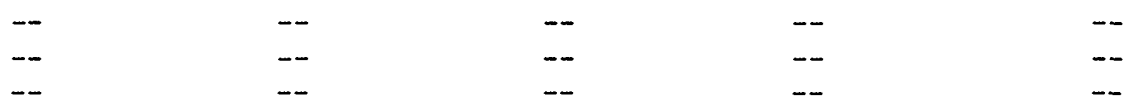

Fox Den, Fever Brook basin at station number 422848072131701

$\begin{array}{rrrrr}-- & 400 & -- & -- & 24 \\ --1 & -- & -- & -- & -- \\ 1.1 & 300 & -- & 1 & 34\end{array}$

Fever Brook basin soil-water sampler at station number 422901072121201

$\begin{array}{rrrrr}4.4 & 100 & -- & 2 & 16 \\ -- & 870 & -- & -- & 8 \\ 8.2 & 1000 & -- & -2 & 34 \\ 6.0 & -- & - & - & 5 \\ -- & 180 & -- & -- & 6\end{array}$

Fever Brook basin deep lysimeter at station number 422909072120901

$\begin{array}{rrrrrr}11 & 430 & -- & 23 & 22 & 50 \\ 12 & -- & -- & 10 & 39 & 13 \\ -- & -- & - & - & 16 & 3 \\ -- & -- & - & -- & -- & --\end{array}$

Fever Brook basin medium lysimeter at station number 422909072121001

$\begin{array}{rrrrr}11 & 600 & -- & 8 & 14 \\ -- & -- & -- & -- & 8\end{array}$




\begin{tabular}{|c|c|c|c|c|c|c|}
\hline Date & $\begin{array}{l}\text { Manga- } \\
\text { nese, } \\
\text { dis- } \\
\text { solved } \\
(\mu g / L \\
\text { as } M n)\end{array}$ & $\begin{array}{c}\text { Mercury, } \\
\text { dis- } \\
\text { solved } \\
(\mu g / L \\
\text { as } H g)\end{array}$ & $\begin{array}{l}\text { Sele- } \\
\text { nium, } \\
\text { dis- } \\
\text { solved } \\
\text { ( } \mu \text { g/L } \\
\text { as Se) }\end{array}$ & $\begin{array}{l}\text { Vana- } \\
\text { dium, } \\
\text { dis- } \\
\text { solved } \\
(\mu \mathrm{g} / \mathrm{L} \\
\text { as V) }\end{array}$ & $\begin{array}{c}\text { Carbon, } \\
\text { organic } \\
\text { total } \\
\text { (mg/L } \\
\text { as C) }\end{array}$ & $\begin{array}{l}\text { Carbon, } \\
\text { organic, } \\
\text { dis- } \\
\text { solved } \\
\text { (mg/L } \\
\text { as C) }\end{array}$ \\
\hline
\end{tabular}

Swift River basin soil-water sampler at station number 422800072232801

$10-15-84$

$10-24-84$

03-11-85

$07-31-85$

$08-15-85$

$08-22-85$

$05-30-85$

$08-02-85$

$08-20-85$

04-07-84

$03-05-85$

$03-26-85$

03-01-84

$03-21-84$

$04-07-84$

$04-18-84$

$04-19-84$

05-29-85

06-28-85

$08-02-85$

08-08-85

$08-20-85$

05-30-85

$08-02-85$

--
49
--

130

$--$

Swift River basin deep lysimeter at station number 422800072233101

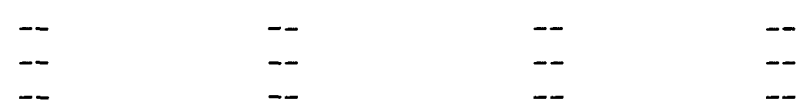

Fox Den, Fever Brook basin at station number 422848072131701

$\begin{array}{llllll}20 & -- & -- & -- & -- & -- \\ -- & -- & -- & -- & -- & --\end{array}$

Fever Brook basin soil-water sampler at station number 422901072121201

$\begin{array}{rlllcl}72 & <0.1 & <1 & <1 & 2.3 & -- \\ 36 & -- & -- & -- & -- \\ 9 & <.1 & <1 & -- & -- & -- \\ 48 & - & - & - & --\end{array}$

Fever Brook basin deep lysimeter at station number 422909072120901

$\begin{array}{rlllll}150 & -- & -- & -- & -- & -- \\ 180 & -- & -- & -- & -- & -- \\ 180 & -- & -- & -- & -- & 2.2 \\ -- & -- & -- & -- & --\end{array}$

Fever Brook basin medium lysimeter at station number 422909072121001

\begin{tabular}{|c|c|c|c|c|c|}
\hline 64 & -- & -- & -- & -- & -- \\
\hline 110 & -- & -- & -- & -- & -- \\
\hline
\end{tabular}

